Supporting Information

\title{
Organocatalytic Asymmetric Nucleophilic Addition to ortho-Quinone Methides by Alcohols
}

Zengwei Lai, Zhaobin Wang, and Jianwei Sun*

Department of Chemistry, the Hong Kong University of Science and Technology, Clear Water Bay, Kowloon, Hong Kong SAR, China

\section{Table of Contents}

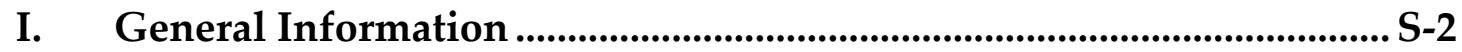

II. Substrate Preparation ................................................................................. S-3

III. Enantioselective C-O Bond Formation ................................................. S-8

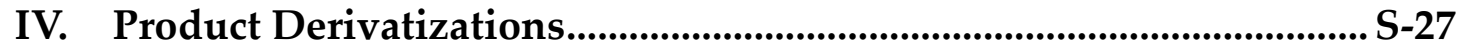

V. Determination of the Product Absolute Stereochemistry …................ S-32

${ }^{1} \mathrm{H}$ NMR and ${ }^{13} \mathrm{C}$ NMR Spectra 


\section{General Information}

All air or moisture sensitive reactions were conducted in oven-dried glassware under nitrogen atmosphere using dry solvents. Flash column chromatography was performed over silica gel (230-400 mesh) purchased from Qindao Puke Co., China. Anhydrous dichloromethane and tetrahydrofuran were purified by the Innovative ${ }^{\circledR}$ solvent purification system. ${ }^{1} \mathrm{H}$ and ${ }^{13} \mathrm{C}$ NMR spectra were collected on a Bruker AV $400 \mathrm{MHz}$ NMR spectrometer using residue solvent peaks as an internal standard $\left({ }^{1} \mathrm{H}\right.$ NMR: $\mathrm{CDCl}_{3}$ at $7.26 \mathrm{ppm},{ }^{13} \mathrm{C} \mathrm{NMR:} \mathrm{CDCl}_{3}$ at $\left.77.0 \mathrm{ppm}\right)$. Mass spectra were collected on an Agilent GC/MS 5975C system, or a MALDI Micro MX mass spectrometer, or an API QSTAR XL System. 


\section{Substrate Preparation}

Substrates $\mathbf{1 a},{ }^{1} \mathbf{1} \mathbf{b},{ }^{2}$ and $\mathbf{1} \mathbf{j}^{3}$ were synthesized following the reported procedures.

\section{General Procedure A. ${ }^{4}$}<smiles>C=Cc1c[R1]ccc1O</smiles>

so

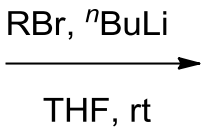

$\mathrm{THF}, \mathrm{rt}$<smiles>[R]C(O)c1c[R1]ccc1O</smiles>

S1

At $-78{ }^{\circ} \mathrm{C}$ under $\mathrm{N}_{2}$, to a flame-dried flask charged with a solution of the $\mathrm{RBr}(10$ $\mathrm{mmol})$ in dry THF (10 mL) was added $n$-BuLi (10 mmol, $2.4 \mathrm{M}$ in hexane, $4.2 \mathrm{~mL})$ dropwise. The reaction was stirred for $1 \mathrm{~h}$ at the same temperature and then a solution of the aldehyde $(4 \mathrm{mmol})$ in THF $(2 \mathrm{~mL})$ was added slowly. The reaction mixture was then warmed to room temperature and stirred overnight. Upon completion, the reaction mixture was cooled to $0{ }^{\circ} \mathrm{C}$ and treated with saturated aqueous $\mathrm{NH}_{4} \mathrm{Cl}$ solution $(20 \mathrm{~mL})$. The mixture was extracted with EtOAc $(3 \times 30 \mathrm{~mL})$. The combined organic layers were dried over $\mathrm{Na}_{2} \mathrm{SO}_{4}$, filtered, and concentrated. The residue was purified by silica gel chromatography to afford the pure product.<smiles>Cc1ccc(O)c(C(O)c2ccccc2)c1</smiles>

2-(Hydroxy(phenyl)methyl)-4-methylphenol (1c) was prepared as white solid according to the General Procedure A (purification by flash column chromatography:

1. Diao, L.; Wan, P. Can. J. Chem. 2008, 86, 105.

2. Wang, P.; Hu, H.; Wang, Y. Org. Lett. 2007, 9, 1533.

3. Alaimo, P. J.; Knight, Z. A.; Shokat, K. M. Bioorg. Med. Chem. 2005, 13, 2825.

4. Zhao, W.; Wang, Z.; Chu, B.; Sun, J. Angew. Chem., Int. Ed. 2015, 54, 1910. 
hexanes/EtOAc $=3: 1)$.

${ }^{1} \mathbf{H}$ NMR (400 MHz, $\left.\mathrm{CDCl}_{3}\right) \delta 7.68(\mathrm{~s}, 1 \mathrm{H}), 7.41-7.30(\mathrm{~m}, 5 \mathrm{H}), 6.99(\mathrm{dd}, J=8.2,1.5 \mathrm{~Hz}$, 1H), $6.79(\mathrm{~d}, J=8.2 \mathrm{~Hz}, 1 \mathrm{H}), 6.69(\mathrm{~s}, 1 \mathrm{H}), 5.95(\mathrm{~s}, 1 \mathrm{H}), 3.03(\mathrm{~d}, J=2.6 \mathrm{~Hz}, 1 \mathrm{H}), 2.21(\mathrm{~s}$, $3 \mathrm{H})$.

${ }^{13} \mathrm{C}$ NMR (100 MHz, $\left.\mathrm{CDCl}_{3}\right) \delta$ 153.0, 141.9, 129.7, 129.1, 128.7, 128.6, 128.1, 126.8, 126.3, 117.1, 77.0, 20.5.

IR (neat, cm-1) 3699, 2918, 1499, 1246, 1017, 699.

HRMS (EI+) calculated for $\mathrm{C}_{14} \mathrm{H}_{14} \mathrm{O}_{2}[\mathrm{M}]^{+}:$214.0994, found 214.0994.

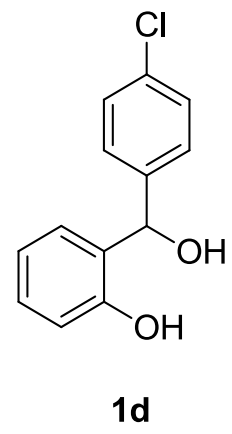

2-((4-Chlorophenyl)(hydroxy)methyl)phenol (1d) was prepared as white solid according to the General Procedure A (purification by flash column chromatography: hexanes/EtOAc $=3: 1)$.

${ }^{1} \mathrm{H}$ NMR $\left(400 \mathrm{MHz} \mathrm{CDCl}_{3}\right) \delta 7.70(\mathrm{~s}, 1 \mathrm{H}), 7.33-7.26(\mathrm{~m}, 4 \mathrm{H}), 7.20(\mathrm{t}, J=8.4 \mathrm{~Hz} 1 \mathrm{H})$, 6.89-6.82 (m, 3H), 5.93 (s, 1H), 3.35 (br s, 1H).

${ }^{13} \mathrm{C}$ NMR $\left(100 \mathrm{MHz}, \mathrm{CDCl}_{3}\right) \delta 155.0,140.3,133.9,129.5,128.7,128.11,128.09,126.6$, $120.2,117.2,75.8$.

IR (neat, cm-1) 3698, 2884, 1489, 1235, 1091, 1011, 756.

HRMS (EI+) calculated for $\mathrm{C}_{13} \mathrm{H}_{11} \mathrm{ClO}_{2}[\mathrm{M}]^{+}:$234.0441, found 234.0448. 
<smiles>Oc1ccccc1C(O)c1ccc(-c2ccccc2)cc1</smiles>

1e

2-([1,1'-Biphenyl]-4-yl(hydroxy)methyl)phenol (1e) was prepared as white solid according to the General Procedure A (purification by flash column chromatography: hexanes/EtOAc $=3: 1)$.

${ }^{1}$ H NMR (400 MHz, $\left.\mathrm{CDCl}_{3}\right) \delta 7.87(\mathrm{~s}, 1 \mathrm{H}), 7.59(\mathrm{t}, J=7.3 \mathrm{~Hz}, 4 \mathrm{H}), 7.48-7.42(\mathrm{~m}, 4 \mathrm{H})$, $7.35(\mathrm{t}, J=7.3 \mathrm{~Hz}, 1 \mathrm{H}), 7.22(\mathrm{t}, J=7.8 \mathrm{~Hz}, 1 \mathrm{H}), 6.93(\mathrm{t}, J=6.5 \mathrm{~Hz}, 2 \mathrm{H}), 6.85(\mathrm{t}, J=7.4 \mathrm{~Hz}$, $1 \mathrm{H}), 6.07(\mathrm{~d}, J=2.3 \mathrm{~Hz}, 1 \mathrm{H}), 2.95(\mathrm{~d}, J=3.1 \mathrm{~Hz}, 1 \mathrm{H})$.

${ }^{13} \mathrm{C}$ NMR (100 MHz, $\left.\mathrm{CDCl}_{3}\right) \delta$ 155.4, 141.1, 140.8, 140.5, 129.3, 128.8, 128.2, 127.4, 127.2 (2C), 127.1, 126.6, 112.0, 117.3, 76.7.

IR (neat, $\left.\mathrm{cm}^{-1}\right)$ 3698, 2883, 1487, 1260, 756.

HRMS (EI+) calculated for $\mathrm{C}_{19} \mathrm{H}_{16} \mathrm{O}_{2}[\mathrm{M}]^{+}:$276.1150, found 276.1215.<smiles>CSc1ccc(C(O)c2ccccc2O)cc1</smiles>

$1 f$

2-(Hydroxy(4-(methylthio)phenyl)methyl)phenol (1f) was prepared as white solid according to the General Procedure A (purification by flash column chromatography: hexanes/EtOAc $=3: 1)$.

${ }^{1} \mathrm{H}$ NMR $\left(400 \mathrm{MHz}^{\mathrm{CDCl}}\right) \delta 7.88(\mathrm{~s}, 1 \mathrm{H}), 7.29(\mathrm{~d}, J=8.3 \mathrm{~Hz}, 2 \mathrm{H}), 7.25-7.15(\mathrm{~m}, 3 \mathrm{H})$, 6.93- $6.84(\mathrm{~m}, 2 \mathrm{H}), 6.82(\mathrm{t}, J=7.3 \mathrm{~Hz}, 1 \mathrm{H}), 5.95(\mathrm{~s}, 1 \mathrm{H}), 3.13$ (br s, 1H), $2.47(\mathrm{~s}, 3 \mathrm{H})$. 
${ }^{13} \mathrm{C}$ NMR $\left(100 \mathrm{MHz}_{\mathrm{CDCl}}\right) \delta$ 155.3, 138.61, 138.57, 129.3, 128.2, 127.3, 126.6, 126.5, $119.9,117.2,76.5,15.6$.

IR (neat, cm-1) 3700, 2920, 1490, 1250, 1080, 757.

HRMS (EI+) calculated for $\mathrm{C}_{14} \mathrm{H}_{14} \mathrm{O}_{2} \mathrm{~S}[\mathrm{M}]^{+}:$246.0715, found 246.0727.

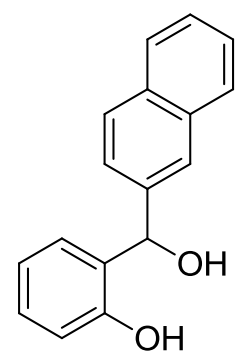

$1 \mathrm{~g}$

2-(Hydroxy(naphthalen-2-yl)methyl)phenol (1g) was prepared as white solid according to the General Procedure A (purification by flash column chromatography: hexanes/EtOAc $=3: 1)$.

${ }^{1} \mathbf{H}$ NMR $\left(400 \mathrm{MHz}, \mathrm{CDCl}_{3}\right) \delta 7.98(\mathrm{~s}, 1 \mathrm{H}), 7.92-7.70(\mathrm{~m}, 4 \mathrm{H}), 7.51-7.49(\mathrm{~m}, 3 \mathrm{H}), 7.20(\mathrm{t}$, $J=7.7 \mathrm{~Hz}, 1 \mathrm{H}), 6.90(\mathrm{dd}, J=11.0,7.4 \mathrm{~Hz}, 2 \mathrm{H}), 6.81(\mathrm{t}, J=7.4 \mathrm{~Hz}, 1 \mathrm{H}), \quad 6.15(\mathrm{~d}, J=1.8$ $\mathrm{Hz}, 1 \mathrm{H}), 3.21(\mathrm{~s}, 1 \mathrm{H})$.

${ }^{13} \mathrm{C}$ NMR $\left(100 \mathrm{MHz} \mathrm{CDCl}_{3}\right) \delta 155.5,139.2,133.14,133.10,129.3,128.6,128.4,128.1$, $127.7,126.5,126.33,126.29,125.6,124.7,119.9,117.2,76.7$.

IR (neat, $\mathrm{cm}^{-1}$ ) 3703, 3053, 1588, 1489, 1237, 1017, 753.

HRMS (EI+) calculated for $\mathrm{C}_{17} \mathrm{H}_{14} \mathrm{O}_{2}[\mathrm{M}]^{+}:$250.0994, found 250.1005. 


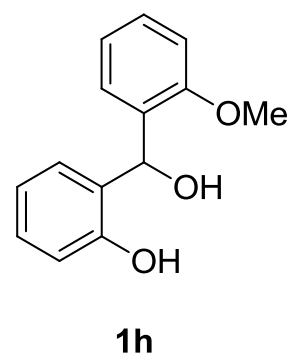

2-(Hydroxy(2-methoxyphenyl)methyl)phenol (1h) was prepared as white solid according to the General Procedure A (purification by flash column chromatography: hexanes/EtOAc $=3: 1)$.

${ }^{1}$ H NMR (400 MHz, $\left.\mathrm{CDCl}_{3}\right) \delta 8.32(\mathrm{~s}, 1 \mathrm{H}), 7.35-7.31(\mathrm{~m}, 1 \mathrm{H}), 7.26-7.21(\mathrm{~m}, 1 \mathrm{H})$, 6.99-6.92 (m, 4H), 6.83-6.79 (m, 2H), $6.22(\mathrm{~s}, 1 \mathrm{H}), 4.09$ (br s, 1H), $3.91(\mathrm{~s}, 3 \mathrm{H})$.

${ }^{13} \mathrm{C}$ NMR $\left(100 \mathrm{MHz}, \mathrm{CDCl}_{3}\right) \delta 156.9,156.4,129.5,129.3,129.2,128.4,127.9,125.2,121.2$, $119.6,117.1,110.7,73.8,55.5$.

IR (neat, cm $\mathrm{cm}^{-1}$ ) 3700, 2940, 1587, 1490, 1243, 1026, 909, 755.

HRMS (EI+) calculated for $\mathrm{C}_{14} \mathrm{H}_{14} \mathrm{O}_{3}[\mathrm{M}]^{+}:$230.0950, found 230.0943.

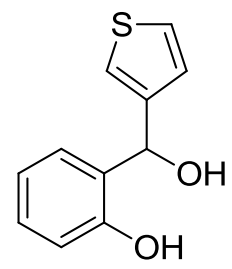

$1 \mathbf{i}$

2-(Hydroxy(thiophen-3-yl)methyl)phenol (1i) was prepared as white solid according to the General Procedure A (purification by flash column chromatography: hexanes/EtOAc $=3: 1)$.

${ }^{1} \mathbf{H}$ NMR $\left(400 \mathrm{MHz} \mathrm{CDCl}_{3}\right) \delta 7.80(\mathrm{~s}, 1 \mathrm{H}), 7.36-7.29(\mathrm{~m}, 1 \mathrm{H}), 7.25-7.16(\mathrm{~m}, 2 \mathrm{H}), 7.07$ (d, $J=5.0 \mathrm{~Hz}, 1 \mathrm{H}), 6.95(\mathrm{~d}, J=7.5 \mathrm{~Hz}, 1 \mathrm{H}), 6.90(\mathrm{~d}, J=8.1 \mathrm{~Hz}, 1 \mathrm{H}), 6.84(\mathrm{t}, J=7.4 \mathrm{~Hz}, 1 \mathrm{H})$, $6.04(\mathrm{~s}, 1 \mathrm{H}), 3.09$ (br s, 1H).

${ }^{13} \mathrm{C}$ NMR (100 MHz, $\left.\mathrm{CDCl}_{3}\right) \delta$ 155.3, 143.2, 129.4, 127.9, 126.7, 126.4, 126.3, 122.4, 119.9, 117.3, 73.3 .

IR (neat, $\left.\mathrm{cm}^{-1}\right) 3698,2892,1488,1235,755$.

HRMS (EI+) calculated for $\mathrm{C}_{11} \mathrm{H}_{10} \mathrm{O}_{2} \mathrm{~S}[\mathrm{M}]^{+}:$206.0402, found 206.0399. 


\section{Enantioselective C-O Bond Formation}

\section{General Procedure B.}

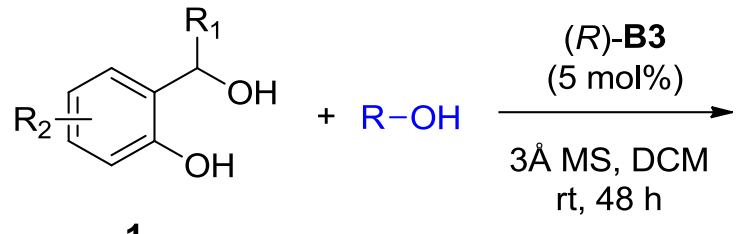

1<smiles>[R]C([R])c1cc[R4]([H])cc1O</smiles>

2

At room temperature (or otherwise noted), to an oven-dried 25-mL flask charged with a mixture of the substrate $\mathbf{1}(0.3 \mathrm{mmol})$, the alcohol $(0.36 \mathrm{mmol})$ and $3 \AA$ molecular sieves $(60 \mathrm{mg})$ in DCM $(12 \mathrm{~mL})$ was added the catalyst $(R)-B 3(10 \mathrm{mg}, 15$ $\mu \mathrm{mol}, 5 \mathrm{~mol} \%$ ). The reaction mixture was stirred for $48 \mathrm{~h}$ (or otherwise noted). The reaction mixture was then treated with solid $\mathrm{Na}_{2} \mathrm{CO}_{3}(50 \mathrm{mg})$ and concentrated under reduced pressure. The residue was purified by silica gel chromatography to afford the pure product 2 .

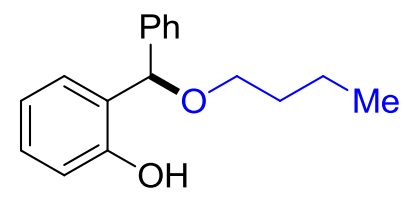

2a

(R)-2-(Butoxy(phenyl)methyl)phenol (2a) was prepared as colorless oil according to the General Procedure B (purification by flash column chromatography: hexanes/EtOAc $=10: 1,68.3 \mathrm{mg}, 89 \%$ yield, 95:5 er).

$[\alpha]^{25}=-83.5\left(\mathrm{c} 1.0, \mathrm{CH}_{2} \mathrm{Cl}_{2}\right.$ ). HPLC analysis of the product: Daicel CHIRALPAK AD-H column; $1 \% i-\mathrm{PrOH}$ in hexanes; $1.0 \mathrm{~mL} / \mathrm{min}$; retention times: $6.8 \mathrm{~min}$ (minor), 7.5 min (major).

${ }^{1} \mathrm{H}$ NMR (400 MHz, $\left.\mathrm{CDCl}_{3}\right) \delta 8.35(\mathrm{~s}, 1 \mathrm{H}), 7.33(\mathrm{~m}, 5 \mathrm{H}), 7.21(\mathrm{t}, J=7.7 \mathrm{~Hz}, 1 \mathrm{H}), 6.91$ $(\mathrm{m}, 2 \mathrm{H}), 6.82(\mathrm{t}, J=7.4 \mathrm{~Hz}, 1 \mathrm{H}), 5.55(\mathrm{~s}, 1 \mathrm{H}), 3.64-3.54(\mathrm{~m}, 2 \mathrm{H}), 1.72-1.65(\mathrm{~m}, 2 \mathrm{H})$, 1.49-1.40 (m, 2H), $0.94(\mathrm{t}, J=7.4 \mathrm{~Hz}, 3 \mathrm{H})$. 
${ }^{13} \mathrm{C}$ NMR $\left(100 \mathrm{MHz}, \mathrm{CDCl}_{3}\right) \delta$ 155.6, 140.2, 129.2, 128.7, 128.5, 128.0, 127.1, 125.0, 119.6, $117.1,85.0,69.5,31.6,19.3,13.7$.

IR $\left(\right.$ neat, cm $\mathrm{cm}^{-1}$ ) 3695, 2960, 2362, 1586, 1488, 1239, 1079, 753.

HRMS (EI+) calculated for $\mathrm{C}_{17} \mathrm{H}_{20} \mathrm{NO}_{2}[\mathrm{M}]^{+}:$256.1463, found 256.1458.<smiles>CCCCOC(c1ccccc1)c1cc(OC)ccc1O</smiles>

2b

(R)-2-(Butoxy(phenyl)methyl)-4-methoxyphenol (2b) was prepared as colorless oil according to the General Procedure B (purification by flash column chromatography: hexanes/EtOAc $=10: 1,55.9 \mathrm{mg}, 65 \%$ yield, $96: 4 \mathrm{er})$.

$[\alpha]^{25}=-36.8\left(\right.$ c $1.0, \mathrm{CH}_{2} \mathrm{Cl}_{2}$ ). HPLC analysis of the product: Daicel CHIRALPAK AD-H column; $1 \%$ - $\mathrm{PrOH}$ in hexanes; $1.0 \mathrm{~mL} / \mathrm{min}$; retention times: $11.8 \mathrm{~min}$ (minor), $16.1 \mathrm{~min}$ (major).

${ }^{1} \mathrm{H}$ NMR $\left(400 \mathrm{MHz}_{\mathrm{CDCl}}\right) \delta 7.87(\mathrm{~s}, 1 \mathrm{H}), 7.36-7.28(\mathrm{~m}, 5 \mathrm{H}), 6.84(\mathrm{~d}, J=8.8 \mathrm{~Hz}, 1 \mathrm{H})$, $6.76(\mathrm{dd}, J=8.8,2.9 \mathrm{~Hz}, 1 \mathrm{H}), 6.47(\mathrm{~d}, J=2.9 \mathrm{~Hz}, 1 \mathrm{H}), 5.48(\mathrm{~s}, 1 \mathrm{H}), 3.70(\mathrm{~s}, 3 \mathrm{H}), 3.63-5.52$ $(\mathrm{m}, 2 \mathrm{H}), 1.70-1.63(\mathrm{~m}, 2 \mathrm{H}), 1.47-1.38(\mathrm{~m}, 2 \mathrm{H}), 0.92(\mathrm{t}, J=7.4 \mathrm{~Hz}, 3 \mathrm{H})$.

${ }^{13} \mathrm{C}$ NMR $\left(101 \mathrm{MHz}, \mathrm{CDCl}_{3}\right) \delta$ 152.7, 149.3, 140.0, 128.5, 128.0, 127.1, 125.8, 117.6, 114.4, $114.0,84.8,69.6,55.6,31.6,19.3,13.8$.

IR (neat, cm-1) 3635, 2958, 1498, 1238, 1079, 809, 700.

HRMS (EI+) calculated for $\mathrm{C}_{18} \mathrm{H}_{22} \mathrm{O}_{3}[\mathrm{M}]^{+}:$286.1569, found 286.1572. 


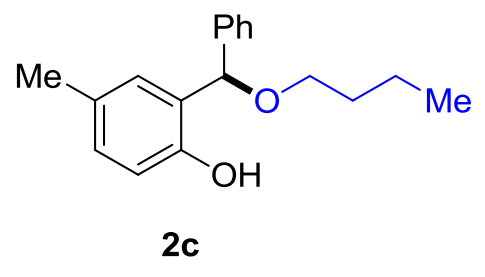

(R)-2-(Butoxy(phenyl)methyl)-4-methylphenol (2c) was prepared as colorless oil according to the General Procedure B (purification by flash column chromatography: hexanes/EtOAc $=10: 1,77.2 \mathrm{mg}, 95 \%$ yield, $94: 6 \mathrm{er}$ ).

$[\alpha]_{\mathrm{D}^{25}}=-40.1\left(\mathrm{c} 1.0, \mathrm{CH}_{2} \mathrm{Cl}_{2}\right)$. HPLC analysis of the product: Daicel CHIRALPAK AD-H column; $1 \%$ i-PrOH in hexanes; $1.0 \mathrm{~mL} / \mathrm{min}$; retention times: $6.7 \mathrm{~min}$ (minor), $8.2 \min$ (major).

${ }^{1} \mathrm{H}$ NMR $\left(400 \mathrm{MHz}, \mathrm{CDCl}_{3}\right) \delta 8.14(\mathrm{~s}, 1 \mathrm{H}), 7.38-7.29(\mathrm{~m}, 5 \mathrm{H}), 7.02(\mathrm{~d}, J=8.1 \mathrm{~Hz}, 1 \mathrm{H})$, $6.83(\mathrm{~d}, J=8.2 \mathrm{~Hz}, 1 \mathrm{H}), 6.73(\mathrm{~s}, 1 \mathrm{H}), 5.50(\mathrm{~s}, 1 \mathrm{H}), 3.63-3.53(\mathrm{~m}, 2 \mathrm{H}), 2.24(\mathrm{~s}, 3 \mathrm{H})$, $1.72-1.65(\mathrm{~m}, 2 \mathrm{H}), 1.50-1.40(\mathrm{dd}, J=14.7,7.4 \mathrm{~Hz}, 2 \mathrm{H}), 0.95(\mathrm{t}, J=7.4 \mathrm{~Hz}, 3 \mathrm{H})$.

${ }^{13}$ C NMR (100 MHz, $\left.\mathrm{CDCl}_{3}\right) \delta$ 153.2, 140.4, 129.6, 129.1, 128.7, 128.5, 127.9, 127.1, 124.7, $116.9,85.1,69.6,31.7,20.4,19.3,13.8$.

IR $\left(\right.$ neat, $\left.\mathrm{cm}^{-1}\right)$ 3381, 1632, 1499, 1258, 1081, 801.

HRMS (EI+) calculated for $\mathrm{C}_{18} \mathrm{H}_{22} \mathrm{O}_{2}[\mathrm{M}]^{+}: 270.1260$, found 270.1618.

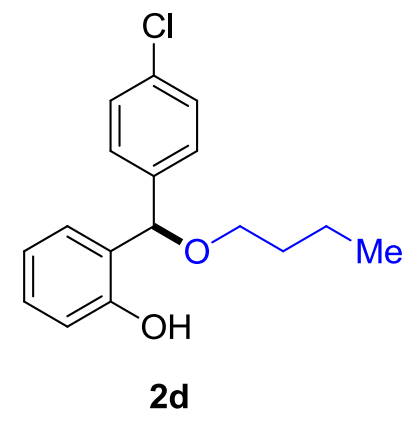

(R)-2-(Butoxy(4-chlorophenyl)methyl)phenol (2d) was prepared as colorless oil according to the General Procedure B (purification by flash column chromatography: hexanes/EtOAc $=10: 1,73.4 \mathrm{mg}, 84 \%$ yield, 96:4 er). 
$[\alpha]_{\mathrm{D}^{25}}=-73.8\left(\mathrm{c} 1.0, \mathrm{CH}_{2} \mathrm{Cl}_{2}\right.$ ). HPLC analysis of the product: Daicel CHIRALPAK AD-H column; $1 \%$-PrOH in hexanes; $1.0 \mathrm{~mL} / \mathrm{min}$; retention times: $8.7 \mathrm{~min}$ (minor), $11.0 \mathrm{~min}$ (major).

${ }^{1}$ H NMR (400 MHz, $\left.\mathrm{CDCl}_{3}\right) \delta 8.13(\mathrm{~s}, 1 \mathrm{H}), 7.33-7.27(\mathrm{~m}, 4 \mathrm{H}), 7.22(\mathrm{t}, J=7.7 \mathrm{~Hz}, 1 \mathrm{H})$, $6.90(\mathrm{dd}, J=7.7,4.0 \mathrm{~Hz}, 2 \mathrm{H}), 6.84(\mathrm{t}, J=7.4 \mathrm{~Hz}, 1 \mathrm{H}), 5.50(\mathrm{~s}, 1 \mathrm{H}), 3.63-3.52(\mathrm{~m}, 2 \mathrm{H})$, $1.48-1.39 \mathrm{~m}, 2 \mathrm{H}), 1.43(\mathrm{~m}, 2 \mathrm{H}), 0.94(\mathrm{t}, J=7.4 \mathrm{~Hz}, 3 \mathrm{H})$.

${ }^{13} \mathrm{C}$ NMR $\left(100 \mathrm{MHz}, \mathrm{CDCl}_{3}\right) \delta$ 155.4, 138.8, 133.8, 129.4, 128.6, 128.6, 128.5, 124.6, 119.8, $117.2,84.1,69.7,31.6,19.3,13.8$.

IR (neat, cm-1) 3326, 2076, 1639, 1488, 1083, 753.

HRMS (EI+) calculated for $\mathrm{C}_{17} \mathrm{H}_{19} \mathrm{ClO}_{2}[\mathrm{M}]^{+}:$290.1074, found 290.1076 .

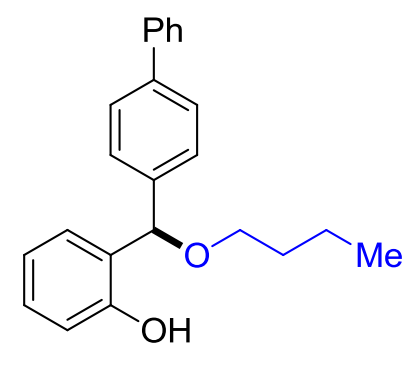

$2 e$

(R)-2-([1,1'-Biphenyl]-4-yl(butoxy)methyl)phenol (2e) was prepared as colorless oil according to the General Procedure B (purification by flash column chromatography: hexanes $/$ EtOAc $=10: 1,89.4 \mathrm{mg}, 90 \%$ yield, 96.5:3.5 er).

$[\alpha]_{\mathrm{D}^{25}}=-81.2\left(\mathrm{c} 1.0, \mathrm{CH}_{2} \mathrm{Cl}_{2}\right.$ ). HPLC analysis of the product: Daicel CHIRALPAK AD-H column; $1 \%$ - $\mathrm{PrOH}$ in hexanes; $1.0 \mathrm{~mL} / \mathrm{min}$; retention times: $12.7 \mathrm{~min}$ (minor), $15.2 \mathrm{~min}$ (major).

${ }^{1}$ H NMR $\left(400 \mathrm{MHz}, \mathrm{CDCl}_{3}\right) \delta 8.39(\mathrm{~s}, 1 \mathrm{H}), 7.64-7.62(\mathrm{~m}, 4 \mathrm{H}), 7.50-7.47(\mathrm{~m}, 4 \mathrm{H}), 7.39$ (t, $J=7.3 \mathrm{~Hz}, 1 \mathrm{H}), 7.27(\mathrm{t}, J=7.5 \mathrm{~Hz}, 1 \mathrm{H}), 7.00-6.98(\mathrm{~m}, 2 \mathrm{H}), 6.89(\mathrm{t}, J=7.4 \mathrm{~Hz}, 1 \mathrm{H}), 5.63(\mathrm{~s}$, $1 \mathrm{H}), 3.72-3.62(\mathrm{q}, J=6.4 \mathrm{~Hz}, 2 \mathrm{H}), 1.78-1.71(\mathrm{~m}, 2 \mathrm{H}), 1.55-1.46(\mathrm{~m}, 2 \mathrm{H}), 1.00(\mathrm{t}, J=7.4$ $\mathrm{Hz}, 3 \mathrm{H})$. 
${ }^{13} \mathrm{C}$ NMR $\left(100 \mathrm{MHz} \mathrm{CDCl}_{3}\right) \delta 155.6,140.9,140.6,139.3,129.2,128.73,128.71,127.6$, 127.33, 127.27, 127.1, 125.0, 119.7, 117.2, 84.8, 69.7, 31.7, 19.3, 13.8.

IR (neat, $\mathrm{cm}^{-1}$ ) 3336, 2076, 1639, 1485, 1259, 1079, 754.

HRMS (EI+) calculated for $\mathrm{C}_{23} \mathrm{H}_{24} \mathrm{O}_{2}[\mathrm{M}]^{+}$: 332.1776, found 332.1767.

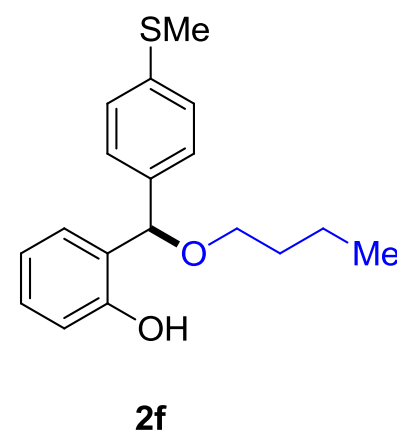

(R)-2-(Butoxy(4-(methylthio)phenyl)methyl)phenol (2f) was prepared as colorless oil according to the General Procedure B (purification by flash column chromatography: hexanes/EtOAc $=10: 1,59.9 \mathrm{mg}, 66 \%$ yield, 95:5 er).

$[\alpha]^{25}=-80.3\left(\mathrm{c} 1.0, \mathrm{CH}_{2} \mathrm{Cl}_{2}\right.$ ). HPLC analysis of the product: Daicel CHIRALPAK AD-H column; $1 \%$ - $\mathrm{PrOH}$ in hexanes; $1.0 \mathrm{~mL} / \mathrm{min}$; retention times: $11.9 \mathrm{~min}$ (minor), $13.8 \mathrm{~min}$ (major).

${ }^{1} \mathrm{H}$ NMR $\left(400 \mathrm{MHz}, \mathrm{CDCl}_{3}\right) \delta 8.33(\mathrm{~s}, 1 \mathrm{H}), 7.31-7.21(\mathrm{~m}, 5 \mathrm{H}), 6.92(\mathrm{dd}, J=11.9,7.9 \mathrm{~Hz}$, 2H), $6.84(\mathrm{t}, J=7.4 \mathrm{~Hz}, 1 \mathrm{H}), 5.53(\mathrm{~s}, 1 \mathrm{H}), 3.64-3.54(\mathrm{~m}, 2 \mathrm{H}), 2.49(\mathrm{~s}, 3 \mathrm{H}), 1.73-1.66(\mathrm{~m}$, $2 \mathrm{H}), 1.50-1.41(\mathrm{~m}, 2 \mathrm{H}), 0.96(\mathrm{t}, J=7.3 \mathrm{~Hz}, 3 \mathrm{H})$.

${ }^{13} \mathrm{C}$ NMR $\left(100 \mathrm{MHz}, \mathrm{CDCl}_{3}\right) \delta$ 155.5, 138.4, 137.0, 129.2, 128.6, 127.7, 126.5, 124.9, 119.6, $117.1,84.6,69.5,31.6,19.3,15.6,13.8$.

IR (neat, $\mathrm{cm}^{-1}$ ) 3328, 2075, 1634, 1083, 754 .

HRMS (EI+) calculated for $\mathrm{C}_{18} \mathrm{H}_{22} \mathrm{O}_{2} \mathrm{~S}[\mathrm{M}]^{+}:$302.1341, found 302.1351. 


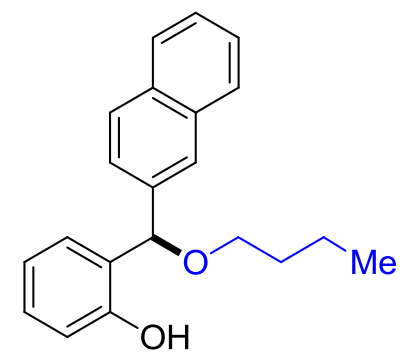

$2 \mathrm{~g}$

(R)-2-(Butoxy(naphthalen-2-yl)methyl)phenol (2g) was prepared as colorless oil according to the General Procedure B (purification by flash column chromatography: hexanes/EtOAc $=10: 1,72.5 \mathrm{mg}, 79 \%$ yield, 95:5 er).

$[\alpha]^{25}=-102.5\left(\mathrm{c} 1.0, \mathrm{CH}_{2} \mathrm{Cl}_{2}\right.$ ). HPLC analysis of the product: Daicel CHIRALPAK AD-H column; $1 \%$-PrOH in hexanes; $1.0 \mathrm{~mL} / \mathrm{min}$; retention times: $13.8 \mathrm{~min}$ (minor), $15.2 \min$ (major).

${ }^{1}$ H NMR $\left(400 \mathrm{MHz}, \mathrm{CDCl}_{3}\right) \delta 8.56$ (s, 1H), 7.90-7.84 (m, 4H), 7.59-7.53 (m, 3H), 7.27 (t, $J=7.2 \mathrm{~Hz}, 1 \mathrm{H}), 7.01(\mathrm{~d}, J=8.0 \mathrm{~Hz}, 1 \mathrm{H}), 6.96(\mathrm{~d}, J=7.2 \mathrm{~Hz}, 1 \mathrm{H}), 6.87(\mathrm{t}, J=7.1 \mathrm{~Hz}, 1 \mathrm{H})$, $5.77(\mathrm{~s}, 1 \mathrm{H}), 3.70-3.60(\mathrm{~m}, 2 \mathrm{H}), 1.79-1.72(\mathrm{~m}, 2 \mathrm{H}), 1.55-1.46(\mathrm{dd}, J=14.3,7.2 \mathrm{~Hz}, 2 \mathrm{H})$, $0.99(\mathrm{t}, J=7.3 \mathrm{~Hz}, 3 \mathrm{H})$.

${ }^{13} \mathrm{C}$ NMR $\left(100 \mathrm{MHz}, \mathrm{CDCl}_{3}\right) \delta$ 155.7, 137.6, 133.1, 133.0, 129.2, 128.7, 128.6, 128.1, 127.6, $126.3,126.21,126.19,125.1,125.0,119.6,117.2,85.2,69.6,31.7,19.3,13.8$.

IR $\left(\right.$ neat, $\left.\mathrm{cm}^{-1}\right)$ 3338, 2959, 2362, 1588, 1486, 1239, 1078, 751.

HRMS (EI+) calculated for $\mathrm{C}_{21} \mathrm{H}_{22} \mathrm{O}_{2}[\mathrm{M}]^{+}:$306.1620, found 306.1620.

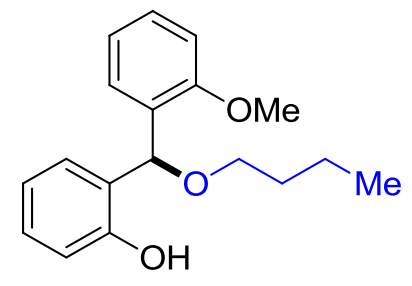

$2 h$

(R)-2-(Butoxy(2-methoxyphenyl)methyl)phenol (2h) was prepared as colorless oil according to the General Procedure B (purification by flash column chromatography: hexanes/EtOAc $=10: 1,66.5 \mathrm{mg}, 78 \%$ yield, 95:5 er). 
$[\alpha]_{\mathrm{D}^{25}}=-153.5\left(\mathrm{c} 1.0, \mathrm{CH}_{2} \mathrm{Cl}_{2}\right.$ ). HPLC analysis of the product: Daicel CHIRALPAK AD-H column; $1 \%$ - $\mathrm{PrOH}$ in hexanes; $0.5 \mathrm{~mL} / \mathrm{min}$; retention times: $14.6 \mathrm{~min}$ (minor), 15.7 min (major).

${ }^{1} \mathrm{H}$ NMR $\left(400 \mathrm{MHz}, \mathrm{CDCl}_{3}\right) \delta 8.56(\mathrm{~s}, 1 \mathrm{H}), 7.38(\mathrm{~d}, J=7.6 \mathrm{~Hz}, 1 \mathrm{H}), 7.31-7.26(\mathrm{~m}, 1 \mathrm{H})$, $7.17(\mathrm{t}, J=7.7 \mathrm{~Hz}, 1 \mathrm{H}), 6.97(\mathrm{t}, J=6.9 \mathrm{~Hz}, 2 \mathrm{H}), 6.92(\mathrm{dd}, J=8.2,2.5 \mathrm{~Hz}, 2 \mathrm{H}), 6.80(\mathrm{t}, J=$ $7.4 \mathrm{~Hz}, 1 \mathrm{H}), 6.05(\mathrm{~s}, 1 \mathrm{H}), 3.89(\mathrm{~s}, 3 \mathrm{H}), 3.63-3.54(\mathrm{~m}, 2 \mathrm{H}), 1.70-1.63(\mathrm{~m}, 2 \mathrm{H}), 1.48-1.38(\mathrm{~m}$, $2 \mathrm{H}), 0.93(\mathrm{t}, J=7.4 \mathrm{~Hz}, 3 \mathrm{H})$.

${ }^{13} \mathrm{C}$ NMR $\left(100 \mathrm{MHz}, \mathrm{CDCl}_{3}\right) \delta 156.7,155.6,129.1,128.73,128.68,128.1,127.8,125.4$, $121.0,119.6,116.9,110.6,77.9,69.5,55.4,31.7,19.3,13.8$.

IR (neat, $\mathrm{cm}^{-1}$ ) 3318, 2073, 1624, 1489, 1242, 1030, 753.

HRMS (EI+) calculated for $\mathrm{C}_{18} \mathrm{H}_{22} \mathrm{O}_{3}[\mathrm{M}]^{+}:$286.1569, found 286.1580.

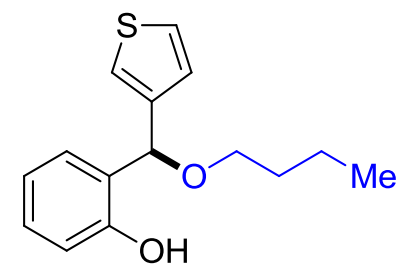

$2 \mathbf{i}$

(S)-2-(Butoxy(thiophen-3-yl)methyl)phenol (2i) was prepared as colorless oil according to the General Procedure B (purification by flash column chromatography: hexanes/EtOAc $=10: 1,54.7 \mathrm{mg}, 70 \%$ yield, 93:7 er).

$[\alpha]^{25}=-62.7$ (c 1.0, $\mathrm{CH}_{2} \mathrm{Cl}_{2}$ ). HPLC analysis of the product: Daicel CHIRALPAK AD-H column; $1 \%$-PrOH in hexanes; $1.0 \mathrm{~mL} / \mathrm{min}$; retention times: $6.7 \mathrm{~min}$ (minor), 7.5 min (major).

${ }^{1} \mathrm{H}$ NMR $\left(400 \mathrm{MHz} \mathrm{CDCl}_{3}\right) \delta 8.18(\mathrm{~s}, 1 \mathrm{H}), 7.29(\mathrm{dd}, J=4.8,3.1 \mathrm{~Hz}, 1 \mathrm{H}), 7.22(\mathrm{t}, J=7.7$ $\mathrm{Hz}, 1 \mathrm{H}), 7.15(\mathrm{~s}, 1 \mathrm{H}), 7.06(\mathrm{~d}, J=5.0 \mathrm{~Hz}, 1 \mathrm{H}), 6.97(\mathrm{~d}, J=7.5 \mathrm{~Hz}, 1 \mathrm{H}), 6.92(\mathrm{~d}, J=8.1 \mathrm{~Hz}$, $1 \mathrm{H}), 6.85(\mathrm{t}, J=7.4 \mathrm{~Hz}, 1 \mathrm{H}), 5.57(\mathrm{~s}, 1 \mathrm{H}), 3.64-3.54(\mathrm{~m}, 2 \mathrm{H}), 1.71-1.64(\mathrm{~m}, 2 \mathrm{H}), 1.48-1.39$ $(\mathrm{m}, 2 \mathrm{H}), 0.94(\mathrm{t}, J=7.4 \mathrm{~Hz}, 3 \mathrm{H})$. 
${ }^{13} \mathrm{C}$ NMR $\left(100 \mathrm{MHz}_{2} \mathrm{CDCl}_{3}\right) \delta$ 155.5, 141.7, 129.3, 128.4, 126.6, 126.3, 124.7, 122.7, 119.7, $117.1,81.3,69.5,31.6,19.3,13.8$.

IR (neat, $\mathrm{cm}^{-1}$ ) 3203, 2076, 1620, 1486, 1240, 1078, 754.

HRMS (EI+) calculated for $\mathrm{C}_{15} \mathrm{H}_{18} \mathrm{O}_{2} \mathrm{~S}[\mathrm{M}]^{+}:$262.1028, found 262.1023.

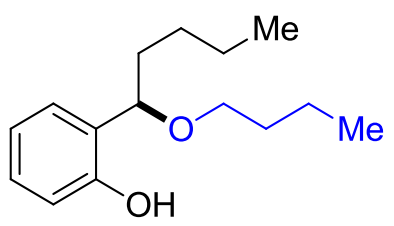

2j

(R)-2-(1-Butoxybutyl)phenol (2j) was prepared as colorless oil according to the General Procedure B (purification by flash column chromatography: hexanes/EtOAc $=10: 1,54.1 \mathrm{mg}, 81 \%$ yield, $85: 15 \mathrm{er}$ ).

$[\alpha]^{25}=+3.2\left(\mathrm{c} 1.0, \mathrm{CH}_{2} \mathrm{Cl}_{2}\right.$ ). HPLC analysis of the product: Daicel CHIRALCEL OJ-H column; $0.5 \%$ - $\mathrm{PrOH}$ in hexanes; $1.0 \mathrm{~mL} / \mathrm{min}$; retention times: $4.3 \mathrm{~min}$ (major), 4.9 $\min ($ minor).

${ }^{1} \mathrm{H}$ NMR $\left(400 \mathrm{MHz}, \mathrm{CDCl}_{3}\right) \delta 8.19(\mathrm{~s}, 1 \mathrm{H}), 7.17(\mathrm{t}, J=7.7 \mathrm{~Hz}, 1 \mathrm{H}), 6.92(\mathrm{~d}, J=7.4 \mathrm{~Hz}$, $1 \mathrm{H}), 6.87-6.70(\mathrm{~m}, 2 \mathrm{H}), 4.31(\mathrm{t}, J=7.6 \mathrm{~Hz}, 1 \mathrm{H}), 3.57-3.51(\mathrm{~m}, 1 \mathrm{H}), 3.44-3.38(\mathrm{~m}, 1 \mathrm{H})$, 1.96-1.87 (m, 1H), 1.74-1.65 (m, 1H), 1.63-1.56 (m, 2H), 1.46-1.20 (m, 6H), 0.91 (t, J = 7.6 $\mathrm{Hz}, 3 \mathrm{H}), 0.88(\mathrm{t}, J=7.8 \mathrm{~Hz}, 3 \mathrm{H})$.

${ }^{13} \mathrm{C}$ NMR $\left(100 \mathrm{MHz}, \mathrm{CDCl}_{3}\right) \delta$ 155.5, 128.7, 128.2, 125.7, 119.4, 116.7, 84.4, 69.7, 35.8, $31.8,27.9,22.4,19.3,13.9,13.8$.

IR (neat, $\mathrm{cm}^{-1}$ ) 3697, 3347, 2958, 1587, 1488, 1242, 1080, 755.

HRMS (EI+) calculated for $\mathrm{C}_{15} \mathrm{H}_{24} \mathrm{O}_{2}[\mathrm{M}]^{+}:$236.1776, found 236.1760. 
<smiles>COC(c1ccccc1)c1ccccc1O</smiles>

2k

(R)-2-(Methoxy(phenyl)methyl)phenol (2k) was prepared as colorless oil according to the General Procedure B (purification by flash column chromatography: hexanes/EtOAc $=10: 1,59.1 \mathrm{mg}, 92 \%$ yield, $86: 14 \mathrm{er})$.

$[\alpha]_{D^{25}}=-56.7$ (c 1.0, $\mathrm{CH}_{2} \mathrm{Cl}_{2}$ ). HPLC analysis of the product: Daicel CHIRALCEL OD-H column; $1 \%$ - $\mathrm{PrOH}$ in hexanes; $1.0 \mathrm{~mL} / \mathrm{min}$; retention times: $10.2 \mathrm{~min}$ (minor), 11.9 min (major).

${ }^{1} \mathrm{H}$ NMR $\left(400 \mathrm{MHz}^{\mathrm{CDCl}} 3\right) \delta 8.08(\mathrm{~s}, 1 \mathrm{H}), 7.43-7.27(\mathrm{~m}, 5 \mathrm{H}), 7.21(\mathrm{t}, J=7.7 \mathrm{~Hz}, 1 \mathrm{H})$, $6.92(\mathrm{dd}, J=7.9,3.1 \mathrm{~Hz}, 2 \mathrm{H}), 6.84(\mathrm{t}, J=7.4 \mathrm{~Hz}, 1 \mathrm{H}), 5.46(\mathrm{~s}, 1 \mathrm{H}), 3.49(\mathrm{~s}, 3 \mathrm{H})$.

${ }^{13} \mathrm{C}$ NMR (100 MHz, $\left.\mathrm{CDCl}_{3}\right) \delta$ 155.4, 139.7, 129.3, 128.8, 128.6, 128.2, 127.2, 124.7, 119.7, 117.1, 86.6, 57.2.

IR (neat, $\left.\mathrm{cm}^{-1}\right)$ 3355, 2961, 1590, 1489, 1236, 1073, 754.

HRMS (EI+) calculated for $\mathrm{C}_{14} \mathrm{H}_{14} \mathrm{O}_{2}[\mathrm{M}]^{+}:$214.0994, found 214.0996.<smiles>CCOC(c1ccccc1)c1ccccc1O</smiles>

2)

(R)-2-(Ethoxy(phenyl)methyl)phenol (21) was prepared as colorless oil according to the General Procedure B (purification by flash column chromatography: hexanes/EtOAc $=10: 1,60.7 \mathrm{mg}, 89 \%$ yield, 93:7 er).

$[\alpha]^{25}=-80.3\left(\mathrm{c} 1.0, \mathrm{CH}_{2} \mathrm{Cl}_{2}\right.$ ). HPLC analysis of the product: Daicel CHIRALPAK AD-H column; $1 \%$-PrOH in hexanes; $0.5 \mathrm{~mL} / \mathrm{min}$; retention times: $15.9 \mathrm{~min}$ (minor), $17.6 \min$ (major). 
${ }^{1} \mathbf{H}$ NMR (400 MHz, $\left.\mathrm{CDCl}_{3}\right) \delta 8.35(\mathrm{~s}, 1 \mathrm{H}), 7.39-7.30(\mathrm{~m}, 5 \mathrm{H}), 7.22(\mathrm{t}, J=7.7 \mathrm{~Hz}, 1 \mathrm{H})$, 6.94-6.91 (m, 2H), $6.84(\mathrm{t}, J=7.4 \mathrm{~Hz}, 1 \mathrm{H}), 5.58(\mathrm{~s}, 1 \mathrm{H}), 3.72-3.61(\mathrm{~m}, 2 \mathrm{H}), 1.34(\mathrm{t}, J=7.0$ $\mathrm{Hz}, 3 \mathrm{H})$.

${ }^{13} \mathrm{C}$ NMR (100 MHz, $\left.\mathrm{CDCl}_{3}\right) \delta$ 155.6, 140.2, 129.2, 128.7, 128.5, 128.0, 127.2, 125.1, 119.7, $117.2,84.8,65.2,15.1$.

IR (neat, cm ${ }^{-1}$ ) 3696, 2975, 1489, 1240, 1066, 754.

HRMS (EI+) calculated for $\mathrm{C}_{15} \mathrm{H}_{16} \mathrm{O}_{2}[\mathrm{M}]^{+}:$228.1150, found 228.1149.<smiles>Oc1ccccc1C(OCc1ccccc1)c1ccccc1</smiles>

(R)-2-((benzyloxy)(phenyl)methyl)phenol (2m) was prepared as colorless oil according to the General Procedure B (purification by flash column chromatography: hexanes $/$ EtOAc $=10: 1,72.7 \mathrm{mg}, 84 \%$ yield, 92.5:7.5 er).

$[\alpha]^{25}=-49.6\left(\mathrm{c} 1.0, \mathrm{CH}_{2} \mathrm{Cl}_{2}\right)$. HPLC analysis of the product: Daicel CHIRALCEL OD-H column; 2\% $i$-PrOH in hexanes; $1.0 \mathrm{~mL} / \mathrm{min}$; retention times: $15.1 \mathrm{~min}$ (minor), 17.7 min (major).

${ }^{1}$ H NMR (400 MHz, $\left.\mathrm{CDCl}_{3}\right) \delta 8.04(\mathrm{~s}, 1 \mathrm{H}), 7.42-7.30(\mathrm{~m}, 10 \mathrm{H}), 7.26-7.22(\mathrm{~m}, 1 \mathrm{H}), 6.94$ $(\mathrm{d}, J=9.2 \mathrm{~Hz}, 2 \mathrm{H}), 6.86(\mathrm{t}, J=7.4 \mathrm{~Hz}, 1 \mathrm{H}), 5.66(\mathrm{~s}, 1 \mathrm{H}), 4.67\left(\mathrm{AB}, J_{A B}=11.5 \mathrm{~Hz}, 1 \mathrm{H}\right)$, $4.62\left(\mathrm{~d}, J_{A B}=11.5 \mathrm{~Hz}, 1 \mathrm{H}\right)$.

${ }^{13} \mathrm{C}$ NMR $\left(100 \mathrm{MHz}, \mathrm{CDCl}_{3}\right) \delta$ 155.5, 139.8, 136.7, 129.4, 129.0, 128.62, 128.57, 128.23, $128.23,128.21,127.3,124.7,119.8,117.2,83.5,71.2$.

IR (neat, cm-1) 3403, 1618, 1489, 1237, 1056, 755.

HRMS (EI+) calculated for $\mathrm{C}_{20} \mathrm{H}_{18} \mathrm{O}_{2}[\mathrm{M}]^{+}:$290.1307, found 290.1299 . 


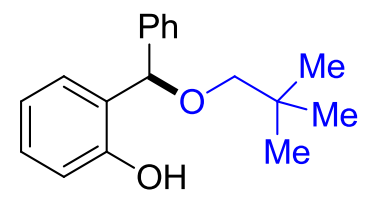

2n

(R)-2-((Neopentyloxy)(phenyl)methyl)phenol (2n) was prepared as colorless oil according to the General Procedure B (purification by flash column chromatography: hexanes/EtOAc $=10: 1,52.8 \mathrm{mg}, 65 \%$ yield, 92.5:7.5 er). This compound solidifies upon standing in the refrigerator.

$[\alpha]^{25}=-36.1$ (c 1.0, $\mathrm{CH}_{2} \mathrm{Cl}_{2}$ ). HPLC analysis of the product: Daicel CHIRALPAK AD-H column; $0.5 \%$ - $\mathrm{PrOH}$ in hexanes; $0.5 \mathrm{~mL} / \mathrm{min}$; retention times: $11.7 \mathrm{~min}$ (minor), $12.7 \mathrm{~min}$ (major).

${ }^{1} \mathrm{H}$ NMR $\left(400 \mathrm{MHz}^{\mathrm{CDCl}} 3\right) \delta 8.26(\mathrm{~s}, 1 \mathrm{H}), 7.37-7.29(\mathrm{~m}, 5 \mathrm{H}), 7.21(\mathrm{t}, J=8.4 \mathrm{~Hz}, 1 \mathrm{H})$, $6.92(\mathrm{~d}, J=8.1 \mathrm{~Hz}, 1 \mathrm{H}), 6.89-6.87(\mathrm{~m}, 1 \mathrm{H}), 6.82(\mathrm{t}, J=7.4 \mathrm{~Hz}, 1 \mathrm{H}), 5.52(\mathrm{~s}, 1 \mathrm{H}), 3.28(\mathrm{AB}$, $\left.J_{A B}=8.7 \mathrm{~Hz}, 1 \mathrm{H}\right), 3.21\left(\mathrm{BA}, J_{B A}=8.7 \mathrm{~Hz}, 1 \mathrm{H}\right), 0.99(\mathrm{~s}, 9 \mathrm{H})$.

${ }^{13} \mathrm{C}$ NMR (100 MHz, $\left.\mathrm{CDCl}_{3}\right) \delta$ 155.6, 140.2, 129.2, 128.7, 128.5, 128.0, 127.2, 125.2, 119.6, $117.1,85.5,80.3,32.0,26.7$.

IR (neat, $\mathrm{cm}^{-1}$ ) 3697, 2961, 1486, 1261, 1030, 800.

HRMS (EI+) calculated for $\mathrm{C}_{18} \mathrm{H}_{22} \mathrm{O}_{2}[\mathrm{M}]^{+}:$270.1620, found 270.1608.<smiles>C=CCOC(c1ccccc1)c1ccccc1O</smiles>

20

(R)-2-((Allyloxy)(phenyl)methyl)phenol (2o) was prepared as colorless oil according to the General Procedure B (purification by flash column chromatography: hexanes/EtOAc $=10: 1,55.5 \mathrm{mg}, 77 \%$ yield, 94:6 er).

$[\alpha]^{25}=-67.4\left(\mathrm{c} 1.0, \mathrm{CH}_{2} \mathrm{Cl}_{2}\right)$. HPLC analysis of the product: Daicel CHIRALPAK IC column; $0.5 \%$ i-PrOH in hexanes; $1.0 \mathrm{~mL} / \mathrm{min}$; retention times: $16.9 \mathrm{~min}$ (minor), 18.0 
$\min$ (major).

${ }^{1} \mathbf{H}$ NMR $\left(400 \mathrm{MHz}, \mathrm{CDCl}_{3}\right) \delta 8.07$ (s, 1H), 7.39-7.30 (m, 5H), 7.23-7.20 (m, 1H), 6.93 (d, $J=8.0 \mathrm{~Hz}, 2 \mathrm{H}), 6.84(\mathrm{t}, J=7.4 \mathrm{~Hz}, 1 \mathrm{H}), 6.01-5.95(\mathrm{~m}, 1 \mathrm{H}), 5.64(\mathrm{~s}, 1 \mathrm{H}), 5.34(\mathrm{dd}, J=17.2$, $1.4 \mathrm{~Hz}, 1 \mathrm{H}), 5.28(\mathrm{dd}, J=10.4,1.2 \mathrm{~Hz}, 1 \mathrm{H}), 4.12(\mathrm{dd}, J=5.7,1.2 \mathrm{~Hz}, 2 \mathrm{H})$.

${ }^{13} \mathrm{C}$ NMR $\left(100 \mathrm{MHz}, \mathrm{CDCl}_{3}\right) \delta$ 155.5, 139.9, 133.3, 129.3, 128.8, 128.6, 128.1, 127.2, 124.8, $119.8,118.4,117.2,83.8,70.10$.

IR (neat, $\left.\mathrm{cm}^{-1}\right)$ 3386, 2361, 1587, 1489, 1239, 1053, 755.

HRMS (EI+) calculated for $\mathrm{C}_{16} \mathrm{H}_{16} \mathrm{O}_{2}[\mathrm{M}]^{+}:$240.1150, found 240.1156.<smiles>C=C(C)COC(c1ccccc1)c1ccccc1O</smiles>

$2 p$

(R)-2-(((2-Methylallyl)oxy)(phenyl)methyl)phenol (2p) was prepared as colorless oil according to the General Procedure B (purification by flash column chromatography: hexanes/EtOAc $=10: 1,68.8 \mathrm{mg}, 90 \%$ yield, 94.5:5.5 er).

$[\alpha]_{D^{25}}=-64.4\left(\right.$ c $1.0, \mathrm{CH}_{2} \mathrm{Cl}_{2}$ ). HPLC analysis of the product: Daicel CHIRALPAK AD-H column; $1 \%$-PrOH in hexanes; $0.5 \mathrm{~mL} / \mathrm{min}$; retention times: $19.4 \mathrm{~min}$ (minor), 20.8 min (major).

${ }^{1}$ H NMR (400 MHz, $\left.\mathrm{CDCl}_{3}\right) \delta 8.11(\mathrm{~s}, 1 \mathrm{H}), 7.39-7.28(\mathrm{~m}, 5 \mathrm{H}), 7.24-7.19(\mathrm{~m}, 1 \mathrm{H})$, 6.94-6.91 (m, 2H), 6.86-6.82 (m, 1H), $5.60(\mathrm{~s}, 1 \mathrm{H}), 5.02(\mathrm{~s}, 1 \mathrm{H}), 4.99(\mathrm{~s}, 1 \mathrm{H}), 4.04\left(\mathrm{AB}, J_{A B}\right.$ $=12.3 \mathrm{~Hz}, 1 \mathrm{H}), 3.99\left(\mathrm{BA}, J_{B A}=12.3 \mathrm{~Hz}, 1 \mathrm{H}\right), 1.81(\mathrm{~s}, 3 \mathrm{H})$.

${ }^{13} \mathrm{C}$ NMR $\left(100 \mathrm{MHz}_{2} \mathrm{CDCl}_{3}\right) \delta$ 155.5, 140.8, 139.9, 129.3, 128.9, 128.6, 128.1, 127.2, 124.7, $119.7,117.2,113.6,83.6,73.1,19.7$.

IR (neat, cm-1) 3701, 2964, 1586, 1489, 1240, 1030, 755.

HRMS (EI+) calculated for $\mathrm{C}_{17} \mathrm{H}_{18} \mathrm{O}_{2}[\mathrm{M}]^{+}:$254.1307, found 254.1304 . 


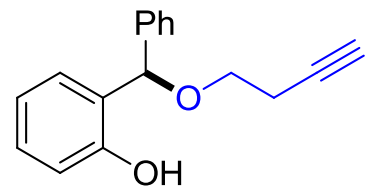

$2 q$

(R)-2-((But-3-yn-1-yloxy)(phenyl)methyl)phenol (2q) was prepared as colorless oil according to the General Procedure B (purification by flash column chromatography: hexanes/EtOAc $=10: 1,57.3 \mathrm{mg}, 76 \%$ yield, 96:4 er).

$[\alpha]_{\mathrm{D}^{25}}=-72.7$ (c 1.0, $\mathrm{CH}_{2} \mathrm{Cl}_{2}$ ). HPLC analysis of the product: Daicel CHIRALCEL OD-H column; $1 \%$ - $\mathrm{PrOH}$ in hexanes; $1.0 \mathrm{~mL} / \mathrm{min}$; retention times: $13.0 \mathrm{~min}$ (minor), $16.8 \mathrm{~min}$ (major).

${ }^{1} \mathbf{H}$ NMR $\left(400 \mathrm{MHz}, \mathrm{CDCl}_{3}\right) \delta 7.95(\mathrm{~s}, 1 \mathrm{H}), 7.39-7.30(\mathrm{~m}, 5 \mathrm{H}), 7.23-7.18(\mathrm{~m}, 1 \mathrm{H}), 6.93(\mathrm{~d}$, $J=8.8 \mathrm{~Hz}, 1 \mathrm{H}), 6.85-6.79(\mathrm{~m}, 2 \mathrm{H}), 5.63(\mathrm{~s}, 1 \mathrm{H}), 3.71-3.63(\mathrm{~m}, 2 \mathrm{H}), 2.58-2.54(\mathrm{~m}, 2 \mathrm{H})$, $2.09(\mathrm{t}, J=2.6 \mathrm{~Hz}, 1 \mathrm{H})$.

${ }^{13} \mathrm{C}$ NMR $\left(100 \mathrm{MHz}, \mathrm{CDCl}_{3}\right) \delta$ 155.5, 139.5, 129.4, 128.7, 128.6, 128.3, 127.4, 124.9, 119.7, $117.3,84.9,80.6,70.1,67.3,19.8$.

IR $\left(\right.$ neat, cm $\mathrm{cm}^{-1}$ ) 3294, 2877, 2361, 1586, 1489, 1237, 1086, 756.

HRMS (EI+) calculated for $\mathrm{C}_{17} \mathrm{H}_{16} \mathrm{O}_{2}[\mathrm{M}]^{+}:$252.1150, found 252.1151.<smiles>CC(C)OC(c1ccccc1)c1ccccc1O</smiles>

(R)-2-(Isopropoxy(phenyl)methyl)phenol (2r) was prepared as colorless oil according to the General Procedure B (purification by flash column chromatography: hexanes $/$ EtOAc $=10: 1,52.4 \mathrm{mg}, 72 \%$ yield, 94.5:5.5 er).

$[\alpha]^{25}=-78.4\left(\mathrm{c} 1.0, \mathrm{CH}_{2} \mathrm{Cl}_{2}\right.$ ). HPLC analysis of the product: Daicel CHIRALPAK AD-H column; $1 \%$-PrOH in hexanes; $1.0 \mathrm{~mL} / \mathrm{min}$; retention times: $13.0 \mathrm{~min}$ (major), $13.9 \min ($ minor). 
${ }^{1} \mathbf{H}$ NMR $\left(400 \mathrm{MHz} \mathrm{CDCl}_{3}\right) \delta 8.46(\mathrm{~d}, J=5.0 \mathrm{~Hz}, 1 \mathrm{H}), 7.38-7.29(\mathrm{~m}, 5 \mathrm{H}), 7.24-7.21(\mathrm{~m}$, $1 \mathrm{H}), 6.93(\mathrm{~d}, J=3.1 \mathrm{~Hz}, 2 \mathrm{H}), 6.87-6.83(\mathrm{~m}, 1 \mathrm{H}), 5.69(\mathrm{~d}, J=4.8 \mathrm{~Hz}, 1 \mathrm{H}), 3.90-3.84(\mathrm{~m}$, $1 \mathrm{H}), 1.31(\mathrm{~d}, J=3.4 \mathrm{~Hz}, 6 \mathrm{H})$.

${ }^{13} \mathrm{C}$ NMR $\left(100 \mathrm{MHz}, \mathrm{CDCl}_{3}\right) \delta$ 155.8, 140.6, 129.1, 128.7, 128.5, 127.9, 127.2, 125.4, 119.6, 117.2, 81.9, 70.6, 22.01, 21.97.

IR (neat, $\mathrm{cm}^{-1}$ ) 3352, 2973, 1588, 1489, 1379, 1241, 1179, 1023, 754.

HRMS (EI+) calculated for $\mathrm{C}_{16} \mathrm{H}_{18} \mathrm{O}_{2}[\mathrm{M}]^{+}:$242.1307, found 242.1298.

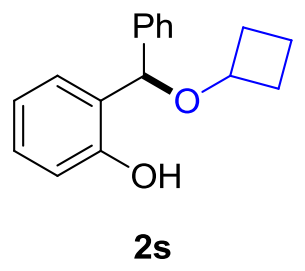

(R)-2-(Cyclobutoxy(phenyl)methyl)phenol (2s) was prepared as colorless oil according to the General Procedure B (purification by flash column chromatography: hexanes/EtOAc $=10: 1,69.1 \mathrm{mg}, 91 \%$ yield, 93:7 er).

$[\alpha]_{\mathrm{D}^{25}}=-82.1$ (c 1.0, $\mathrm{CH}_{2} \mathrm{Cl}_{2}$ ). HPLC analysis of the product: Daicel CHIRALCEL OD-H column; $1 \% i$-PrOH in hexanes; $0.5 \mathrm{~mL} / \mathrm{min}$; retention times: $13.7 \mathrm{~min}$ (minor), 14.7 min (major).

${ }^{1}$ H NMR (400 MHz, $\left.\mathrm{CDCl}_{3}\right) \delta 8.28(\mathrm{~s}, 1 \mathrm{H}), 7.37-7.30(\mathrm{~m}, 5 \mathrm{H}), 7.21(\mathrm{t}, J=7.7 \mathrm{~Hz}, 1 \mathrm{H})$, 6.94-6.90 (m, 2H), $6.83(\mathrm{t}, J=7.4 \mathrm{~Hz}, 1 \mathrm{H}), 5.55(\mathrm{~s}, 1 \mathrm{H}), 4.12(\mathrm{p}, J=7.4 \mathrm{~Hz}, 1 \mathrm{H}), 2.25-2.16$ (m, 2H), 2.14-2.02 (m, 2H), $1.75(\mathrm{q}, J=10.1 \mathrm{~Hz}, 1 \mathrm{H}), 1.54-1.43(\mathrm{~m}, 1 \mathrm{H})$.

${ }^{13} \mathrm{C}$ NMR $\left(100 \mathrm{MHz}, \mathrm{CDCl}_{3}\right) \delta$ 155.6, 140.2, 129.2, 128.6, 128.5, 128.0, 127.3, 125.1, 119.7, $117.2,82.2,72.1,30.6,30.2,12.5$.

IR (neat, $\mathrm{cm}^{-1}$ ) 3360, 2982, 1619, 1489, 1240, 1095, 754.

HRMS (EI+) calculated for $\mathrm{C}_{17} \mathrm{H}_{18} \mathrm{O}_{2}[\mathrm{M}]^{+}:$254.1307, found 254.1310. 


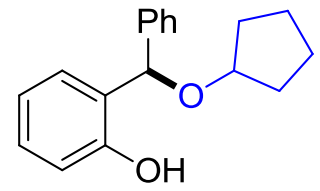

$2 \mathbf{t}$

(R)-2-((Cyclopentyloxy)(phenyl)methyl)phenol (2t) was prepared as colorless oil according to the General Procedure B (purification by flash column chromatography: hexanes/EtOAc $=10: 1,58.5 \mathrm{mg}, 74 \%$ yield, 93:7 er).

$[\alpha]_{D^{25}}=-62.0\left(\mathrm{c} 1.0, \mathrm{CH}_{2} \mathrm{Cl}_{2}\right.$ ). HPLC analysis of the product: Daicel CHIRALCEL OD-H column; $1 \%$-PrOH in hexanes; $0.5 \mathrm{~mL} / \mathrm{min}$; retention times: $11.6 \mathrm{~min}$ (minor), $12.6 \mathrm{~min}$ (major).

${ }^{1} \mathrm{H}$ NMR $\left(400 \mathrm{MHz} \mathrm{CDCl}_{3}\right) \delta 8.43(\mathrm{~s}, 1 \mathrm{H}), 7.35-7.28(\mathrm{~m}, 5 \mathrm{H}), 7.21(\mathrm{t}, J=7.7 \mathrm{~Hz}, 1 \mathrm{H})$, $6.93(\mathrm{t}, J=8.6 \mathrm{~Hz}, 2 \mathrm{H}), 6.84(\mathrm{t}, J=7.4 \mathrm{~Hz}, 1 \mathrm{H}), 5.61(\mathrm{~s}, 1 \mathrm{H}), 4.15(\mathrm{~s}, 1 \mathrm{H}), 1.81(\mathrm{~s}, 6 \mathrm{H})$, $1.60(\mathrm{~d}, J=6.6 \mathrm{~Hz}, 2 \mathrm{H})$.

${ }^{13} \mathrm{C}$ NMR $\left(100 \mathrm{MHz}, \mathrm{CDCl}_{3}\right) \delta$ 155.8, 140.6, 129.2, 128.8, 128.5, 127.9, 127.2, 125.1, 119.6, $117.2,82.5,80.2,32.3,32.2,23.5,23.4$.

IR (neat, $\mathrm{cm}^{-1}$ ) 3355, 2959, 1620, 1489, 1241, 1025, 754 .

HRMS (EI+) calculated for $\mathrm{C}_{18} \mathrm{H}_{20} \mathrm{O}_{2}[\mathrm{M}]^{+}:$268.1463, found 268.1458.<smiles>Oc1ccccc1C(OCCCCl)c1ccccc1</smiles>

$2 \mathrm{u}$

(R)-2-((3-Chloropropoxy)(phenyl)methyl)phenol (2u) was prepared as colorless oil according to the General Procedure B (purification by flash column chromatography: hexanes/EtOAc $=10: 1,73.5 \mathrm{mg}, 89 \%$ yield, 94:6 er). $[\alpha]_{D^{25}}=-54.4\left(\mathrm{c} 1.0, \mathrm{CH}_{2} \mathrm{Cl}_{2}\right.$ ). HPLC analysis of the product: Daicel CHIRALPAK AS-H column; $1 \%$ i-PrOH in hexanes; $1.0 \mathrm{~mL} / \mathrm{min}$; retention times: $16.3 \mathrm{~min}$ (minor), 18.7 min (major). 
${ }^{1} \mathbf{H}$ NMR (400 MHz, $\left.\mathrm{CDCl}_{3}\right) \delta 7.81(\mathrm{~s}, 1 \mathrm{H}), 7.40-7.27(\mathrm{~m}, 5 \mathrm{H}), 7.22(\mathrm{t}, J=7.5 \mathrm{~Hz}, 1 \mathrm{H})$, $6.92(\mathrm{t}, J=8.8 \mathrm{~Hz}, 2 \mathrm{H}), 6.84(\mathrm{t}, J=7.4 \mathrm{~Hz}, 1 \mathrm{H}), 5.57(\mathrm{~s}, 1 \mathrm{H}), 3.80-3.63(\mathrm{~m}, 4 \mathrm{H}), 2.23-2.00$ $(\mathrm{m}, 2 \mathrm{H})$.

${ }^{13} \mathrm{C}$ NMR $\left(100 \mathrm{MHz}, \mathrm{CDCl}_{3}\right) \delta$ 155.3, 139.7, 129.4, 128.8, 128.6, 128.2, 127.1, 124.8, 119.9, $117.1,85.0,66.2,41.5,32.4$.

IR (neat, $\left.\mathrm{cm}^{-1}\right)$ 3337, 2360, 1587, 1488, 1239, 1093, 755.

HRMS (EI+) calculated for $\mathrm{C}_{16} \mathrm{H}_{17} \mathrm{ClO}_{2}[\mathrm{M}]^{+}:$276.0917, found 276.0928.

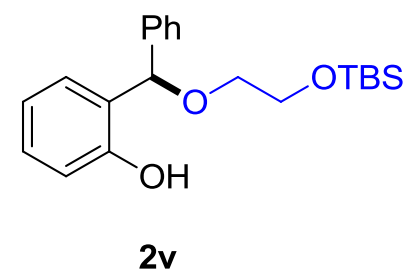

(R)-2-((2-((tert-Butyldimethylsilyl)oxy)ethoxy)(phenyl)methyl)phenol (2v) was prepared as colorless oil according to the General Procedure B (purification by flash column chromatography: hexanes/EtOAc $=10: 1,76.4 \mathrm{mg}, 71 \%$ yield, 97:3 er).

$[\alpha]_{\mathrm{D}^{25}}=-44.3\left(\mathrm{c} 1.0, \mathrm{CH}_{2} \mathrm{Cl}_{2}\right.$ ). HPLC analysis of the product: Daicel CHIRALCEL OJ-H column; $1 \%$ i-PrOH in hexanes; $1.0 \mathrm{~mL} / \mathrm{min}$; retention times: $6.4 \mathrm{~min}$ (major), 11.2 $\min ($ minor).

${ }^{1} \mathbf{H}$ NMR $\left(400 \mathrm{MHz} \mathrm{CDCl}_{3}\right) \delta 8.17(\mathrm{~s}, 1 \mathrm{H}), 7.43-7.29(\mathrm{~m}, 5 \mathrm{H}), 7.17(\mathrm{~d}, J=7.2 \mathrm{~Hz}, 1 \mathrm{H})$, $6.93(\mathrm{~d}, J=8.1 \mathrm{~Hz}, 1 \mathrm{H}), 6.75(\mathrm{~d}, J=7.3 \mathrm{~Hz}, 1 \mathrm{H}), 6.69(\mathrm{~d}, J=7.5 \mathrm{~Hz}, 1 \mathrm{H}), 5.64(\mathrm{~s}, 1 \mathrm{H})$, 3.89-3.79 (m, 2H), 3.67-3.58 (m, 2H), $0.92(\mathrm{~s}, 9 \mathrm{H}), 0.10(\mathrm{~s}, 6 \mathrm{H})$.

${ }^{13} \mathrm{C}$ NMR (100 MHz, $\left.\mathrm{CDCl}_{3}\right) \delta$ 156.0, 139.5, 129.2, 128.5 (2C), 128.1, 127.6, 125.7, 119.4, $117.4,84.5,70.7,62.2,25.9,18.3,-5.39,-5.42$.

IR (neat, $\mathrm{cm}^{-1}$ ) 3645, 3173, 2954, 2360, 1615, 1486, 1256, 1107, 833.

HRMS (EI+) calculated for $\mathrm{C}_{21} \mathrm{H}_{30} \mathrm{O}_{3} \mathrm{Si}[\mathrm{M}]^{+}$: 358.1964, found 358.1978. 


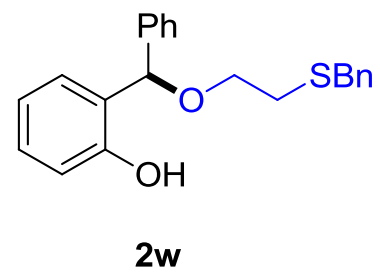

(R)-2-((2-(Benzylthio)ethoxy)(phenyl)methyl)phenol (2w) was prepared as colorless oil according to the General Procedure B (purification by flash column chromatography: hexanes/EtOAc $=10: 1,77.4 \mathrm{mg}, 74 \%$ yield, 96.5:3.5 er).

$[\alpha]_{\mathrm{D}^{25}}=-52.3$ (c 1.0, $\mathrm{CH}_{2} \mathrm{Cl}_{2}$ ). HPLC analysis of the product: Daicel CHIRALCEL OD-H column; 3\% i-PrOH in hexanes; $1.0 \mathrm{~mL} / \mathrm{min}$; retention times: $21.1 \mathrm{~min}$ (minor), $23.0 \mathrm{~min}$ (major).

${ }^{1} \mathrm{H}$ NMR $\left(400 \mathrm{MHz}, \mathrm{CDCl}_{3}\right) \delta 8.03(\mathrm{~s}, 1 \mathrm{H}), 7.43-7.19(\mathrm{~m}, 11 \mathrm{H}), 6.97(\mathrm{~d}, J=8.1 \mathrm{~Hz}, 1 \mathrm{H})$, $6.83(\mathrm{~d}, J=4.5 \mathrm{~Hz}, 2 \mathrm{H}), 5.56(\mathrm{~s}, 1 \mathrm{H}), 3.75(\mathrm{~s}, 2 \mathrm{H}), 3.70-3.51(\mathrm{~m}, 2 \mathrm{H}), 2.71(\mathrm{t}, J=6.3 \mathrm{~Hz}$, $2 \mathrm{H})$.

${ }^{13} \mathrm{C}$ NMR $\left(100 \mathrm{MHz}, \mathrm{CDCl}_{3}\right) \delta 155.5,139.5,137.9,129.4,128.8,128.7,128.6,128.5,128.2$, $127.3,127.1,125.0,119.7,117.3,84.8,67.8,36.3,30.7$.

IR $\left(\right.$ neat, $\left.\mathrm{cm}^{-1}\right)$ 3625, 3215, 2360, 2075, 1632, 1488, 1072, 753.

HRMS (EI+) calculated for $\mathrm{C}_{22} \mathrm{H}_{22} \mathrm{O}_{2} \mathrm{~S}[\mathrm{M}]^{+}: 350.1341$, found 350.1328 .<smiles>O=C(OCCOC(c1ccccc1)c1ccccc1O)c1ccccc1</smiles>

(R)-2-((2-Hydroxyphenyl)(phenyl)methoxy)ethyl benzoate (2x) was prepared as colorless oil according to the General Procedure B (purification by flash column chromatography: hexanes $/ \mathrm{EtOAc}=10: 1,73.8 \mathrm{mg}, 71 \%$ yield, 95.5:4.5 er).

$[\alpha]_{\mathrm{D}^{25}}=-51.1$ (c 1.0, $\mathrm{CH}_{2} \mathrm{Cl}_{2}$ ). HPLC analysis of the product: Daicel CHIRALCEL OD-H column; 10\% $i$-PrOH in hexanes; $1.0 \mathrm{~mL} / \mathrm{min}$; retention times: $9.3 \mathrm{~min}$ (minor), $11.0 \mathrm{~min}$ (major). 
${ }^{1} \mathrm{H}$ NMR $\left(400 \mathrm{MHz}^{\mathrm{CDCl}}\right)_{3} \delta 8.11(\mathrm{~d}, J=7.3 \mathrm{~Hz}, 2 \mathrm{H}), 7.95(\mathrm{~s}, 1 \mathrm{H}), 7.59(\mathrm{t}, J=7.4 \mathrm{~Hz}$, $1 \mathrm{H}), 7.48(\mathrm{t}, J=7.6 \mathrm{~Hz}, 2 \mathrm{H}), 7.42-7.27(\mathrm{~m}, 5 \mathrm{H}), 7.24-7.12(\mathrm{~m}, 1 \mathrm{H}), 6.92(\mathrm{~d}, J=8.1 \mathrm{~Hz}$, $1 \mathrm{H}), 6.87(\mathrm{~d}, J=6.3 \mathrm{~Hz}, 1 \mathrm{H}), 6.82(\mathrm{t}, J=7.4 \mathrm{~Hz}, 1 \mathrm{H}), 5.66(\mathrm{~s}, 1 \mathrm{H}), 4.62-4.57(\mathrm{~m}, 1 \mathrm{H}), 4.56$ $-4.45(\mathrm{~m}, 1 \mathrm{H}), 3.97-3.79(\mathrm{~m}, 2 \mathrm{H})$.

${ }^{13} \mathrm{C}$ NMR (100 MHz, $\left.\mathrm{CDCl}_{3}\right) \delta$ 166.4, 155.5, 139.3, 133.1, 129.8, 129.7, 129.5, 128.8, 128.6, $128.4,128.3,127.3,124.8,119.8,117.3,84.9,67.4,63.6$.

IR (neat, cm-1) 3626, 3194, 2360, 1631, 1275, 753.

HRMS (EI+) calculated for $\mathrm{C}_{22} \mathrm{H}_{20} \mathrm{O}_{4}[\mathrm{M}]^{+}:$348.1362, found 348.1349.<smiles>COCCOC(c1ccccc1)c1ccccc1O</smiles>

$2 y$

(R)-2-((2-Methoxyethoxy)(phenyl)methyl)phenol (2y) was prepared as colorless oil according to the General Procedure B (purification by flash column chromatography: hexanes/EtOAc $=10: 1,50.4 \mathrm{mg}, 65 \%$ yield, 95:5 er).

$[\alpha]_{D^{25}}=-85.0$ (c 1.0, $\mathrm{CH}_{2} \mathrm{Cl}_{2}$ ). HPLC analysis of the product: Daicel CHIRALPAK AD-H column; $1 \%$ - $\mathrm{PrOH}$ in hexanes; $1.0 \mathrm{~mL} / \mathrm{min}$; retention times: $12.7 \mathrm{~min}$ (minor), $13.5 \mathrm{~min}$ (major).

${ }^{1} \mathrm{H}$ NMR $\left(400 \mathrm{MHz}^{\mathrm{CDCl}} 3\right) \delta 8.24(\mathrm{~s}, 1 \mathrm{H}), 7.61-7.29(\mathrm{~m}, 5 \mathrm{H}), 7.20(\mathrm{t}, J=7.7 \mathrm{~Hz}, 1 \mathrm{H})$, $6.96(\mathrm{~d}, J=8.1 \mathrm{~Hz}, 1 \mathrm{H}), 6.77(\mathrm{t}, J=7.4 \mathrm{~Hz}, 1 \mathrm{H}), 6.68(\mathrm{~d}, J=7.6 \mathrm{~Hz}, 1 \mathrm{H}), 5.67(\mathrm{~s}, 1 \mathrm{H})$, 3.77-3.67 (m, 2H), 3.66-3.56 (m, 2H), $3.46(\mathrm{~s}, 3 \mathrm{H})$.

${ }^{13} \mathrm{C}$ NMR $\left(100 \mathrm{MHz}, \mathrm{CDCl}_{3}\right) \delta$ 155.9, 139.2, 129.3, 128.5 (2C), 128.1, 127.6, 125.8, 119.5, $117.2,83.9,71.2,68.2,58.9$.

IR (neat, $\mathrm{cm}^{-1}$ ) 3685, 2924, 1581, 1485, 1238, 1071.

HRMS (EI-) calculated for $\mathrm{C}_{16} \mathrm{H}_{17} \mathrm{O}_{3}\left[\mathrm{M}-\mathrm{H}^{+}\right]$: 257.1178, found 257.1194. 
<smiles>O=C1c2ccccc2C(=O)N1CCOC(c1ccccc1)c1ccccc1O</smiles>

$2 z$

(R)-2-(2-((2-Hydroxyphenyl)(phenyl)methoxy)ethyl)isoindoline-1,3-dione (2z) was prepared as colorless oil according to the General Procedure B (purification by flash column chromatography: hexanes/EtOAc $=10: 1,78.4 \mathrm{mg}, 70 \%$ yield, 99:1 er). $[\alpha]_{D^{25}}=-69.3\left(\mathrm{c} 1.0, \mathrm{CH}_{2} \mathrm{Cl}_{2}\right.$ ). HPLC analysis of the product: Daicel CHIRALPAK AD-H column; $10 \%$-PrOH in hexanes; $1.0 \mathrm{~mL} / \mathrm{min}$; retention times: $26.9 \mathrm{~min}$ (major), $28.9 \mathrm{~min}$ (minor).

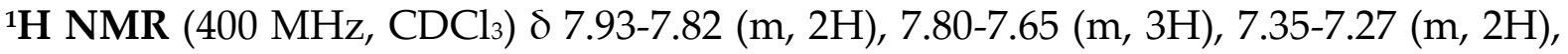
7.27-7.20 (m, 3H), 7.20-7.10 (m, 1H), $6.94(\mathrm{~d}, J=6.6 \mathrm{~Hz}, 1 \mathrm{H}), 6.86(\mathrm{~d}, J=7.9 \mathrm{~Hz}, 1 \mathrm{H})$, $6.80(\mathrm{t}, J=7.4 \mathrm{~Hz}, 1 \mathrm{H}), 5.54(\mathrm{~s}, 1 \mathrm{H}), 4.01(\mathrm{dd}, J=8.1,3.4 \mathrm{~Hz}, 2 \mathrm{H}), 3.92-3.75(\mathrm{~m}, 2 \mathrm{H})$. ${ }^{13} \mathrm{C}$ NMR (100 MHz, $\left.\mathrm{CDCl}_{3}\right) \delta$ 168.2, 154.9, 139.8, 133.9, 131.9, 129.2, 128.6, 128.5, 128.0, $126.91,124.89,123.4,119.8,117.5,85.0,66.6,37.6$.

IR (neat, $\left.\mathrm{cm}^{-1}\right)$ 3629, 3185, 2360, 1709, 1456, 1028, 756.

HRMS (EI+) calculated for $\mathrm{C}_{23} \mathrm{H}_{19} \mathrm{NO}_{4}[\mathrm{M}]^{+}:$373.1314, found 373.1324. 


\section{Product Derivatizations}<smiles>CCCCOC(c1ccc(Cl)cc1)c1ccccc1O</smiles>

2d (96:4 er)<smiles>CCCCOC(C1=CC(OC)(OC)C=CC1=O)c1ccc(Cl)cc1</smiles>

$3(77 \%, 96: 4$ er $)$

(R)-2-(Butoxy(4-chlorophenyl)methyl)-4,4-dimethoxycyclohexa-2,5-dienone (3). A modified literature procedure was followed. ${ }^{5}$ At $0{ }^{\circ} \mathrm{C}$, to the solution of $2 \mathrm{~d}(43.5 \mathrm{mg}$, $0.15 \mathrm{mmol})$ in $\mathrm{MeOH}(3 \mathrm{~mL})$ was added $\mathrm{PhI}(\mathrm{OAc}) 2(106 \mathrm{mg}, 0.33 \mathrm{mmol})$. The mixture was stirred at room temperature for $1 \mathrm{~h}$. Next, saturated aqueous $\mathrm{NaHCO}_{3}$ solution $(5 \mathrm{~mL})$ was added. The mixture was extracted with DCM $(3 \times 10 \mathrm{~mL})$. The combined organic layers were dried over $\mathrm{Na}_{2} \mathrm{SO}_{4}$, filtered, and concentrated. The residue was purified by silica gel chromatography (hexanes/EtOAc $=10: 1$ ) to afford pure 3 as a colorless oil (39.7 mg, 76\% yield, $96: 4 \mathrm{er})$.

$[\alpha]_{\mathrm{D}^{25}}=-169.8 .0\left(\mathrm{c} 1.0, \mathrm{CH}_{2} \mathrm{Cl}_{2}\right.$ ). HPLC analysis of the product: Daicel CHIRALPAK IC column; $1 \%$ - $\mathrm{PrOH}$ in hexanes; $0.5 \mathrm{~mL} / \mathrm{min}$; retention times: $17.1 \mathrm{~min}$ (major), 18.6 $\min$ (minor).

${ }^{1} \mathrm{H}$ NMR $\left(400 \mathrm{MHz}, \mathrm{C}_{6} \mathrm{D}_{6}\right) \delta 7.28(\mathrm{~d}, J=8.2 \mathrm{~Hz}, 2 \mathrm{H}), 7.14(\mathrm{~d}, J=3.2 \mathrm{~Hz}, 1 \mathrm{H}), 7.07(\mathrm{~d}, J=$ $8.2 \mathrm{~Hz}, 2 \mathrm{H}), 6.30(\mathrm{dd}, J=10.3,3.2 \mathrm{~Hz}, 1 \mathrm{H}), 5.96(\mathrm{~d}, J=10.3 \mathrm{~Hz}, 1 \mathrm{H}), 5.38$ (s, 1H), 3.18 (t, $J=6.4 \mathrm{~Hz}, 2 \mathrm{H}), 3.08(\mathrm{~s}, 3 \mathrm{H}), 2.95(\mathrm{~s}, 3 \mathrm{H}), 1.46-1.40(\mathrm{~m}, 2 \mathrm{H}), 1.30-1.21(\mathrm{~m}, 2 \mathrm{H}), 0.79(\mathrm{t}, J$ $=7.3 \mathrm{~Hz}, 3 \mathrm{H})$.

${ }^{13} \mathrm{C}$ NMR (100 MHz, C6 $\left.\mathrm{D}_{6}\right) \delta$ 183.8, 142.9, 141.1, 139.5, 137.8, 133.7, 129.7, 129.1, 128.74, $93.2,76.3,69.0,49.8(2 \mathrm{C}), 32.1,19.7,14.0$.

IR (neat, $\mathrm{cm}^{-1}$ ) 3679, 2958, 1681, 1489, 1371, 1233, 1118, 826.

HRMS (EI+) calculated for $\mathrm{C}_{19} \mathrm{H}_{23} \mathrm{ClO}_{4}[\mathrm{M}]^{+}:$350.1285, found 350.1285.

5. Yin, Z.; Zhang, J.; Wu, J.; Liu, C.; Sioson, K.; Devany, M.; Hu, C.; Zheng, S. Org. Lett. 2013, 15, 3534. 
<smiles>CCCCOC(c1ccc(-c2ccccc2)cc1)c1ccccc1O</smiles>

$2 e(96.5: 3.5 \mathrm{er})$

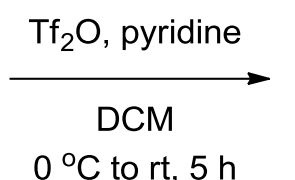

2e' $(85 \%, 97: 3 \mathrm{er})$

(R)-2-([1,1'-Biphenyl]-4-yl(butoxy)methyl)phenyl trifluoromethanesulfonate (2e').

At $0{ }^{\circ} \mathrm{C}$, to a stirred solution of $2 \mathrm{e}(55 \mathrm{mg}, 0.17 \mathrm{mmol})$ in DCM $(3 \mathrm{~mL})$ was added pyridine $(27 \mu \mathrm{L}, 0.34 \mathrm{mmol})$ and $\mathrm{Tf}_{2} \mathrm{O}(204 \mu \mathrm{L}, 1 \mathrm{M}$ in $\mathrm{DCM})$. The mixture was warmed to room temperature and stirred for $5 \mathrm{~h}$. The solvent was removed under reduced pressure, and the residue was purified by silica gel chromatography (hexanes/EtOAc $=20: 1)$ to afford triflate $2 \mathbf{e}^{\prime}$ as a colorless oil $(67.1 \mathrm{mg}, 85 \%$ yield, $97: 3$ er).

$[\alpha]^{25}=+42.7\left(\mathrm{c} 1.0, \mathrm{CH}_{2} \mathrm{Cl}_{2}\right)$. HPLC analysis of the product: Daicel CHIRALPAK IC column; $0.5 \%$ i-PrOH in hexanes; $1.0 \mathrm{~mL} / \mathrm{min}$; retention times: $7.4 \mathrm{~min}$ (minor), 7.7 $\min ($ major).

${ }^{1} \mathrm{H}$ NMR $\left(400 \mathrm{MHz}, \mathrm{CDCl}_{3}\right) \delta 7.66(\mathrm{~d}, J=7.5 \mathrm{~Hz}, 1 \mathrm{H}), 7.59$ (d, J = 7.4 Hz, 4H), 7.43 (dd, $J=14.0,6.0 \mathrm{~Hz}, 5 \mathrm{H}), 7.36(\mathrm{dd}, J=15.8,8.4 \mathrm{~Hz}, 2 \mathrm{H}), 7.29(\mathrm{~d}, J=8.0 \mathrm{~Hz}, 1 \mathrm{H}), 5.76(\mathrm{~s}, 1 \mathrm{H})$, $3.55(\mathrm{t}, J=6.5 \mathrm{~Hz}, 2 \mathrm{H}), 1.76-1.61(\mathrm{~m}, 2 \mathrm{H}), 1.49-1.40(\mathrm{~m}, 2 \mathrm{H}), 0.94(\mathrm{t}, J=7.3 \mathrm{~Hz}, 3 \mathrm{H})$.

${ }^{13} \mathrm{C}$ NMR (100 MHz, $\left.\mathrm{CDCl}_{3}\right) \delta$ 147.2, 140.73, 140.71, 139.4, 135.5, 129.26, 129.1, 128.7, 128.6, 127.4, 127.3, 127.2, 127.0, 121.1, 118.5 (q, Jc-F=318.2 Hz), 77.1, 69.4, 31.8, 19.3, 13.8.

IR (neat, $\mathrm{cm}^{-1}$ ) 3692, 2959, 1421, 1248, 1215, 899, 764.

HRMS (EI-) calculated for $\mathrm{C}_{24} \mathrm{H}_{24} \mathrm{~F}_{3} \mathrm{O}_{4} \mathrm{~S}\left[\mathrm{M}-\mathrm{H}^{+}\right]$: 463.1191, found 463.1190 . 
<smiles>CCCOC(c1ccc(-c2ccccc2)cc1)c1ccccc1[O+]</smiles>

$2 e^{\prime}(97: 3$ er $)$

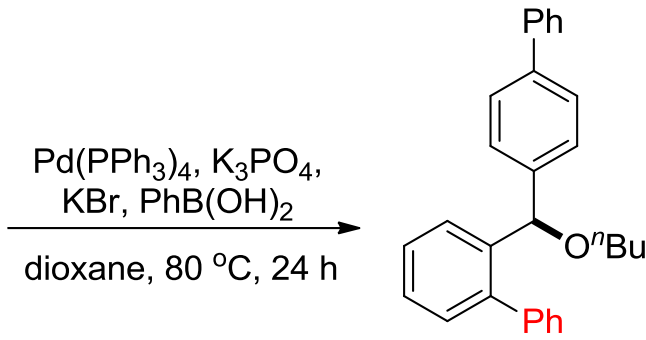

$4(95 \%, 96.5: 3.5 \mathrm{er})$

(R)-2-([1,1'-Biphenyl]-4-yl(butoxy)methyl)-1,1'-biphenyl (4). To a stirred suspension of $\mathrm{Pd}\left(\mathrm{PPh}_{3}\right)_{4}(5.8 \mathrm{mg}, 5 \mu \mathrm{mol}), \mathrm{K}_{3} \mathrm{PO}_{4}(16.0 \mathrm{mg}, 75 \mu \mathrm{mol}), \mathrm{KBr}(6.5 \mathrm{mg}, 55 \mu \mathrm{mol})$, $\mathrm{PhB}(\mathrm{OH})_{2}(12.2 \mathrm{mg}, 0.1 \mathrm{mmol})$ in dioxane $(1 \mathrm{~mL})$ was added a solution of triflate $2 \mathbf{e}^{\prime}(23.2 \mathrm{mg}, 50 \mu \mathrm{mol})$ in dioxane $(1 \mathrm{~mL})$. The reaction was heated at $80{ }^{\circ} \mathrm{C}$ with stirring for $24 \mathrm{~h}$. After cooling to room temperature, the reaction mixture was filtered through a pad of celite. The filtrate was concentrated under reduced pressure, and the residue was purified by silica gel column chromatography (hexanes/EtOAc $=20: 1$ ) to afford 4 as a colorless oil (18.7 $\mathrm{mg}$, 95\% yield, 96.5:3.5 er).

$[\alpha]_{\mathrm{D}^{25}}=+168.4$ (c $1.0, \mathrm{CH}_{2} \mathrm{Cl}_{2}$ ). HPLC analysis of the product: Daicel CHIRALCEL OD-H column; $1 \%$ i-PrOH in hexanes; $1.0 \mathrm{~mL} / \mathrm{min}$; retention times: $4.1 \mathrm{~min}$ (minor), 4.5 min (major).

${ }^{1}$ H NMR (400 MHz, $\left.\mathrm{CDCl}_{3}\right) \delta$ 7.61-7.53 (m, 3H), $7.48(\mathrm{~d}, J=8.2 \mathrm{~Hz}, 2 \mathrm{H})$, 7.43-7.37 (m, $6 \mathrm{H}), 7.35-7.27(\mathrm{~m}, 4 \mathrm{H}), 7.25-7.17(\mathrm{~m}, 3 \mathrm{H}), 5.48(\mathrm{~s}, 1 \mathrm{H}), 3.33(\mathrm{~s}, 2 \mathrm{H}), 1.63-1.49(\mathrm{~m}, 2 \mathrm{H})$, $1.44-1.30(\mathrm{~m}, 2 \mathrm{H}), 0.88(\mathrm{t}, J=7.4 \mathrm{~Hz}, 3 \mathrm{H})$.

${ }^{13} \mathrm{C}$ NMR $\left(100 \mathrm{MHz}, \mathrm{CDCl}_{3}\right) \delta$ 141.9, 141.5, 141.1, 140.9, 139.9, 139.6, 129.8, 129.4, 128.6, 128.0, 127.8, 127.6, 127.4, 127.13, 127.1, 127.0, 126.9, 126.8, 79.3, 68.7, 32.0, 19.4, 13.9. IR (neat, $\left.\mathrm{cm}^{-1}\right)$ 3678, 2957, 1486, 1086, 763.

HRMS (EI+) calculated for $\mathrm{C}_{29} \mathrm{H}_{28} \mathrm{O}[\mathrm{M}]^{+}:$392.2140, found 392.2141. 
<smiles>COCCOC(c1ccccc1)c1ccccc1O</smiles>

2y (95:5 er)

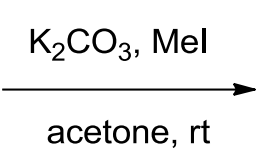

$99 \%$<smiles>COCCOC(c1ccccc1)c1ccccc1OC</smiles>

2y' (95:5 er)

(R)-1-Methoxy-2-((2-methoxyethoxy)(phenyl)methyl)benzene $\left(2 y^{\prime}\right)$. At room temperature, to a solution of $\mathbf{2 y}(35.0 \mathrm{mg}, 0.136 \mathrm{mmol})$ in acetone $(2 \mathrm{~mL})$ was added $\mathrm{K}_{2} \mathrm{CO}_{3}(75.0 \mathrm{mg}, 0.54 \mathrm{mmol})$ and $\mathrm{MeI}(17 \mu \mathrm{L}, 0.272 \mathrm{mmol})$. The mixture was stirred at room temperature for $36 \mathrm{~h}$. Next, $\mathrm{H}_{2} \mathrm{O}(2 \mathrm{~mL})$ was added. The mixture was extracted with $\mathrm{Et}_{2} \mathrm{O}(3 \times 8 \mathrm{~mL})$. The combined organic layers were dried over $\mathrm{Na}_{2} \mathrm{SO}_{4}$, filtered, and concentrated. The residue was purified by silica gel chromatography (hexanes/EtOAc $=20: 1)$ to afford pure $2 \mathbf{y}^{\prime}$ as a colorless oil $(36.6 \mathrm{mg}, 99 \%$ yield, 95:5 er).

$[\alpha]^{25}=+46.1\left(\mathrm{c} 1.0, \mathrm{CH}_{2} \mathrm{Cl}_{2}\right)$. HPLC analysis of the product: Daicel CHIRALPAK IC column; $1 \% \mathrm{i}$-PrOH in hexanes; $1.0 \mathrm{~mL} / \mathrm{min}$; retention times: $9.0 \mathrm{~min}$ (minor), 10.2 $\min ($ major).

${ }^{1} \mathrm{H}$ NMR $\left(400 \mathrm{MHz}, \mathrm{CDCl}_{3}\right) \delta 7.56(\mathrm{~d}, J=7.6 \mathrm{~Hz}, 1 \mathrm{H}), 7.49-7.40(\mathrm{~m}, 2 \mathrm{H}), 7.33$ (t, J = 7.6 $\mathrm{Hz}, 2 \mathrm{H}), 7.25(\mathrm{q}, J=7.5 \mathrm{~Hz}, 2 \mathrm{H}), 7.01(\mathrm{t}, J=7.5 \mathrm{~Hz}, 1 \mathrm{H}), 6.88(\mathrm{~d}, J=8.2 \mathrm{~Hz}, 1 \mathrm{H}), 5.90(\mathrm{~s}$, $1 \mathrm{H}), 3.83(\mathrm{~s}, 3 \mathrm{H}), 3.69-3.64(\mathrm{~m}, 4 \mathrm{H}), 3.43(\mathrm{~s}, 3 \mathrm{H})$.

${ }^{13} \mathrm{C}$ NMR $\left(100 \mathrm{MHz}_{2} \mathrm{CDCl}_{3}\right) \delta 156.5,142.1,130.6,128.2,128.0,127.0$ (2C), 120.7(2C), $110.4,77.2,71.9,68.3,58.9,55.3$.

IR (neat, $\left.\mathrm{cm}^{-1}\right)$ 3648, 3066, 2362, 1579, 1489, 1244, 1089, 754.

HRMS (EI+) calculated for $\mathrm{C}_{17} \mathrm{H}_{20} \mathrm{O}_{3}[\mathrm{M}]^{+}:$272.1412, found 272.1407. 
<smiles>COCCOC(c1ccccc1)c1ccccc1OC</smiles>

2y' (95:5 er)

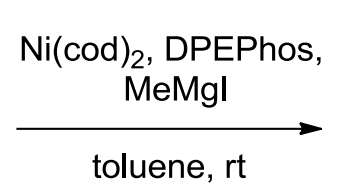

$95 \%$

$92 \%$ enantiospecificity $\mathbf{5}(91.5: 8.5 \mathrm{er})$

(S)-1-Methoxy-2-(1-phenylethyl)benzene (5). A modified literature procedure was followed. ${ }^{6}$ At room temperature, an oven-dried vial was charged with $\mathrm{Ni}(\mathrm{cod})_{2}(3.7$ $\mathrm{mg}, 13.5 \mu \mathrm{mol})$, DPEphos $(14.5 \mathrm{mg}, 27 \mu \mathrm{mol})$ and toluene $(1 \mathrm{~mL})$ in a glovebox. The mixture was stirred at room temperature for $5 \mathrm{~min}$. Next, a solution of $\mathbf{2} \mathbf{y}^{\prime}(36.6 \mathrm{mg}$, $0.135 \mathrm{mmol})$ in toluene $(1 \mathrm{~mL})$ was added followed by dropwise addition of MeMgI $\left(270 \mu \mathrm{L}, 0.27 \mathrm{mmol}, 1 \mathrm{M}\right.$ in $\left.\mathrm{Et}_{2} \mathrm{O}\right)$. The reaction was stirred for $48 \mathrm{~h}$ before quenching with isopropanol $(1 \mathrm{~mL})$. The mixture was filtered through a pad of silica gel, which was washed with $\mathrm{Et}_{2} \mathrm{O}$. The filtrate was concentrated in vacuo and purified by silica gel chromatography (hexanes/EtOAc $=20: 1)$ to afford pure 5 as a colorless oil (27.3 mg, 95\% yield, 91.5:8.5 er).

$[\alpha]_{D^{25}}=-40.5\left(\mathrm{c} 1.0, \mathrm{CH}_{2} \mathrm{Cl}_{2}\right)$. HPLC analysis of the product: Daicel CHIRALCEL OJ-H column; $1 \%$ i-PrOH in hexanes; $1.0 \mathrm{~mL} / \mathrm{min}$; retention times: $9.4 \mathrm{~min}$ (minor), 13.4 $\min$ (major).

${ }^{1} \mathrm{H}$ NMR $\left(400 \mathrm{MHz} \mathrm{CDCl}_{3}\right) \delta$ 7.33-7.24 (m, 4H), 7.24-7.14 (m, 3H), $6.94(\mathrm{t}, J=7.5 \mathrm{~Hz}$, $1 \mathrm{H}), 6.87(\mathrm{~d}, J=8.1 \mathrm{~Hz}, 1 \mathrm{H}), 4.60(\mathrm{q}, J=7.2 \mathrm{~Hz}, 1 \mathrm{H}), 3.80(\mathrm{~s}, 3 \mathrm{H}), 1.61(\mathrm{~d}, J=7.3 \mathrm{~Hz}$, $3 \mathrm{H})$.

${ }^{13} \mathrm{C}$ NMR $\left(100 \mathrm{MHz}, \mathrm{CDCl}_{3}\right) \delta$ 156.8, 146.3, 134.8, 128.0, 127.7, 127.6, 127.0, 125.6, 120.4, $110.5,55.4,37.3,20.8$.

IR (neat, $\mathrm{cm}^{-1}$ ) 3684, 2963, 1593, 1491, 1243, 1029, 699.

HRMS (EI+) calculated for $\mathrm{C}_{15} \mathrm{H}_{16} \mathrm{O}[\mathrm{M}]^{+}:$212.1201, found 212.1195.

6. Greene, M. A.; Yonova, I. M.; Williams, F. J.; Jarvo, E. R. Org. Lett. 2012, 14, 4293. 


\section{Determination of the Product Absolute Stereochemistry}

The absolute stereochemistry of product $\mathbf{2 n}$ was determined by X-ray diffraction. The X-ray data have been deposited at the Cambridge Crystallographic Data Center (CCDC 1414129). The stereochemistry of other products was assumed by analogy.

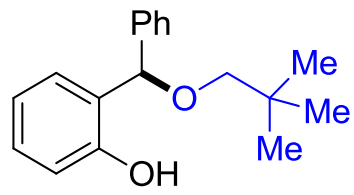

2n

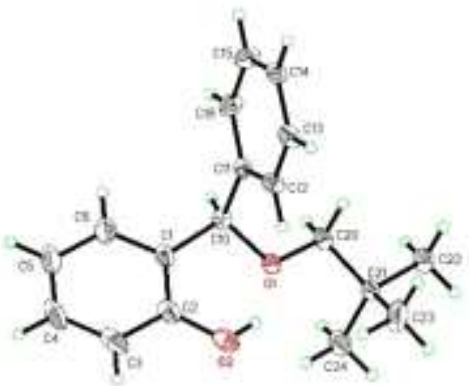

Table 1. Crystal data and structure refinement for $2 \mathbf{n}$.

Identification code 2n

Empirical formula

$\mathrm{C}_{18} \mathrm{H}_{22} \mathrm{O}_{2}$

Formula weight 270.35

Temperature/K 294.04(10)

Crystal system orthorhombic

Space group

$\mathrm{P} 2{ }_{1} 2_{1} 2_{1}$

$\mathrm{a} / \AA \AA$

9.0068(2)

$\mathrm{b} / \AA$

$10.4780(3)$

$\mathrm{c} / \AA$

16.4734(4)

$\alpha{ }^{\circ}$

90

$\beta /{ }^{\circ}$

90

$\gamma /{ }^{\circ}$

90

Volume $/ \AA^{3}$

1554.65(7)

Z

4

$\rho_{\text {calc } g / \mathrm{cm}^{3}}$

1.155

$\mu / \mathrm{mm}^{-1}$

0.576

$\mathrm{F}(000)$

584.0

Crystal size $/ \mathrm{mm}^{3}$

$0.3 \times 0.25 \times 0.1$

Radiation

$\mathrm{CuK} \alpha(\lambda=1.54184)$

$2 \Theta$ range for data collection $/{ }^{\circ} 10.004$ to 134.786

Index ranges

$-9 \leq \mathrm{h} \leq 10,-8 \leq \mathrm{k} \leq 12,-17 \leq 1 \leq 19$ 


$\begin{array}{ll}\text { Reflections collected } & 5580 \\ \text { Independent reflections } & 2764\left[\mathrm{R}_{\text {int }}=0.0296, \mathrm{R}_{\text {sigma }}=0.0434\right] \\ \text { Data/restraints/parameters } & 2764 / 0 / 185 \\ \text { Goodness-of-fit on } \mathrm{F}^{2} & 1.000 \\ \text { Final R indexes [I }>=2 \sigma(\mathrm{I})] & \mathrm{R}_{1}=0.0352, \mathrm{wR}_{2}=0.0835 \\ \text { Final R indexes [all data] } & \mathrm{R}_{1}=0.0424, \mathrm{wR}_{2}=0.0883 \\ \text { Largest diff. peak/hole } / \mathrm{e} \AA^{-3} 0.15 /-0.15 \\ \text { Flack parameter } & 0.02(15)\end{array}$

Table 2. Fractional Atomic Coordinates $\left(\times 10^{4}\right)$ and Equivalent Isotropic Displacement Parameters $\left(\AA^{2} \times 10^{3}\right)$ for $2 n$. Ueq is defined as $1 / 3$ of of the trace of the orthogonalised UIJ tensor.

\begin{tabular}{lrrrr} 
Atom & \multicolumn{1}{c}{$\boldsymbol{x}$} & \multicolumn{1}{l}{$\boldsymbol{z}$} & $\mathbf{U}(\mathbf{e q})$ \\
O1 & $5654.1(15)$ & $3161.3(15)$ & $1870.9(9)$ & $23.1(3)$ \\
O2 & $3665.7(16)$ & $1426.1(16)$ & $1620.9(11)$ & $33.0(4)$ \\
C1 & $3532(2)$ & $2954(2)$ & $2727.2(13)$ & $24.9(5)$ \\
C2 & $2888(2)$ & $2048(2)$ & $2220.3(14)$ & $28.3(5)$ \\
C3 & $1396(3)$ & $1735(3)$ & $2310.2(17)$ & $41.2(7)$ \\
C4 & $547(3)$ & $2323(3)$ & $2901.6(17)$ & $48.8(9)$ \\
C5 & $1160(3)$ & $3232(3)$ & $3397.1(17)$ & $47.7(8)$ \\
C6 & $2649(3)$ & $3552(3)$ & $3308.7(15)$ & $36.3(6)$ \\
C10 & $5172(2)$ & $3303(2)$ & $2697.8(13)$ & $22.7(5)$ \\
C11 & $6093(2)$ & $2515(2)$ & $3286.3(12)$ & $20.8(4)$ \\
C12 & $6399(2)$ & $1240(2)$ & $3139.9(14)$ & $26.0(5)$ \\
C13 & $7197(2)$ & $523(2)$ & $3694.3(15)$ & $28.1(5)$ \\
C14 & $7699(2)$ & $1078(2)$ & $4405.9(14)$ & $26.7(5)$ \\
C15 & $7418(3)$ & $2350(2)$ & $4552.1(14)$ & $33.7(5)$ \\
C16 & $3074(2)$ & $3991.8(13)$ & $29.2(5)$ \\
C20 & $6618(2)$ & $3741(2)$ & $1709.8(14)$ & $27.3(5)$ \\
C21 & $7065(2)$ & $3575(2)$ & $817.8(14)$ & $25.4(5)$ \\
C22 & $7454(2)$ & $4204(3)$ & $685.7(15)$ & $35.8(6)$ \\
C23 & $8966(2)$ & $2163(3)$ & $601.8(18)$ & $46.4(7)$ \\
C24 & $7555(3)$ & $4226(3)$ & $286.9(15)$ & $41.5(7)$
\end{tabular}


Table 3. Anisotropic Displacement Parameters $\left(\AA^{2} \times 10^{3}\right)$ for $2 n$. The Anisotropic displacement factor exponent takes the form: $-2 \pi^{2}\left[h^{2} a * 2 U_{11}+2 h k a * b * U_{12}+\ldots\right]$.

\begin{tabular}{lcccrrr} 
Atom & \multicolumn{1}{c}{$\mathbf{U}_{11}$} & \multicolumn{1}{c}{$\mathbf{U}_{22}$} & \multicolumn{1}{c}{$\mathbf{U}_{33}$} & \multicolumn{1}{c}{$\mathbf{U}_{23}$} & \multicolumn{1}{c}{$\mathbf{U}_{13}$} & \multicolumn{1}{c}{$\mathbf{U}_{12}$} \\
O1 & \multicolumn{1}{c}{$20.1(7)$} & $26.8(8)$ & $22.5(7)$ & $1.6(7)$ & \multicolumn{1}{c}{$2.2(6)$} & \multicolumn{1}{c}{$-5.5(6)$} \\
O2 & $28.5(8)$ & $30.3(9)$ & $40.1(9)$ & $0.7(8)$ & $-6.9(7)$ & $-8.6(7)$ \\
C1 & $20.7(9)$ & $28.8(12)$ & $25.3(10)$ & $10.6(10)$ & $0.8(8)$ & $4.3(9)$ \\
C2 & $23.1(10)$ & $30.6(13)$ & $31.2(11)$ & $14.3(10)$ & $-2.6(9)$ & $-0.1(10)$ \\
C3 & $25.5(11)$ & $51.7(17)$ & $46.4(15)$ & $24.2(14)$ & $-10.5(10)$ & $-8.8(11)$ \\
C4 & $19.0(11)$ & $79(2)$ & $48.5(16)$ & $35.1(16)$ & $3.1(11)$ & $1.9(13)$ \\
C5 & $27.6(11)$ & $79(2)$ & $37.1(13)$ & $21.4(16)$ & $8.8(11)$ & $16.7(14)$ \\
C6 & $30.8(11)$ & $47.3(15)$ & $30.7(12)$ & $11.5(12)$ & $4.9(10)$ & $10.4(11)$ \\
C10 & $23.2(10)$ & $20.9(11)$ & $23.9(11)$ & $1.6(9)$ & $2.1(8)$ & $1.0(9)$ \\
C11 & $18.1(8)$ & $21.5(10)$ & $22.8(10)$ & $2.3(9)$ & $2.0(8)$ & $0.0(8)$ \\
C12 & $24.5(10)$ & $21.8(11)$ & $31.7(12)$ & $-1.5(10)$ & $-7.0(9)$ & $-2.5(9)$ \\
C13 & $24.2(9)$ & $21.4(11)$ & $38.8(13)$ & $1.4(10)$ & $-4.3(9)$ & $0.4(9)$ \\
C14 & $23.7(9)$ & $29.2(12)$ & $27.1(12)$ & $6.3(10)$ & $-1.8(8)$ & $2.9(9)$ \\
C15 & $43.0(13)$ & $34.1(13)$ & $24.1(11)$ & $-2.9(10)$ & $-5.8(11)$ & $5.2(11)$ \\
C16 & $38.5(12)$ & $23.8(11)$ & $25.4(11)$ & $-3.5(10)$ & $-1.0(9)$ & $6.2(10)$ \\
C20 & $20.3(9)$ & $31.5(12)$ & $30.2(12)$ & $0.7(10)$ & $1.5(8)$ & $-7.8(9)$ \\
C21 & $18.9(9)$ & $30.8(12)$ & $26.7(11)$ & $0.7(9)$ & $2.9(8)$ & $-4.5(9)$ \\
C22 & $25.5(11)$ & $47.2(16)$ & $34.9(13)$ & $3.9(12)$ & $5.8(10)$ & $-8.9(11)$ \\
C23 & $37.8(14)$ & $43.1(16)$ & $58.4(17)$ & $-13.4(14)$ & $20.1(13)$ & $-7.3(12)$ \\
C24 & $28.2(12)$ & $63.7(19)$ & $32.5(13)$ & $13.9(13)$ & $2.3(9)$ & $-1.9(12)$
\end{tabular}

Table 4. Bond Lengths for $2 n$.

\begin{tabular}{|c|c|c|c|c|}
\hline \multicolumn{2}{|c|}{ Atom Atom } & \multirow{2}{*}{$\begin{array}{c}\text { Length/̊ } \\
1.437(3)\end{array}$} & Atom Atom & \multirow{2}{*}{$\begin{array}{c}\text { Length } / \AA \\
1.386(3)\end{array}$} \\
\hline $\mathrm{O} 1$ & $\mathrm{C} 10$ & & C11 C12 & \\
\hline $\mathrm{O} 1$ & $\mathrm{C} 20$ & $1.433(2)$ & $\mathrm{C} 11$ & $1.385(3)$ \\
\hline $\mathrm{O} 2$ & $\mathrm{C} 2$ & $1.375(3)$ & C12 C13 & $1.384(3)$ \\
\hline $\mathrm{C} 1$ & $\mathrm{C} 2$ & $1.391(3)$ & $\mathrm{C} 13 \mathrm{C} 14$ & $1.385(3)$ \\
\hline $\mathrm{C} 1$ & C6 & $1.394(3)$ & $\mathrm{C} 14$ & $1.378(3)$ \\
\hline $\mathrm{C} 1$ & $\mathrm{C} 10$ & $1.522(3)$ & $\mathrm{C} 15$ & $1.395(3)$ \\
\hline $\mathrm{C} 2$ & $\mathrm{C} 3$ & $1.391(3)$ & $\mathrm{C} 20$ & $1.520(3)$ \\
\hline $\mathrm{C} 3$ & $\mathrm{C} 4$ & $1.383(4)$ & $\mathrm{C} 21$ & $1.529(3)$ \\
\hline $\mathrm{C} 4$ & C5 & $1.371(5)$ & $\mathrm{C} 21$ & $1.524(3)$ \\
\hline $\mathrm{C} 5$ & C6 & $1.390(4)$ & $\mathrm{C} 21$ & $1.526(3)$ \\
\hline $\mathrm{C} 10$ & $\mathrm{C} 11$ & $1.519(3)$ & & \\
\hline
\end{tabular}


Table 5. Bond Angles for 2n.

\begin{tabular}{lllllllr} 
Atom Atom Atom & \multicolumn{1}{c}{ Angle $^{\circ}$} & \multicolumn{1}{c}{ Atom Atom Atom } & \multicolumn{1}{c}{ Angle ${ }^{\circ}$} \\
$\mathrm{C} 20$ & $\mathrm{O} 1$ & $\mathrm{C} 10$ & $113.57(16)$ & $\mathrm{C} 16$ & $\mathrm{C} 11$ & $\mathrm{C} 10$ & $119.50(19)$ \\
$\mathrm{C} 2$ & $\mathrm{C} 1$ & $\mathrm{C} 6$ & $118.7(2)$ & $\mathrm{C} 16$ & $\mathrm{C} 11$ & $\mathrm{C} 12$ & $119.1(2)$ \\
$\mathrm{C} 2$ & $\mathrm{C} 1$ & $\mathrm{C} 10$ & $123.3(2)$ & $\mathrm{C} 13$ & $\mathrm{C} 12$ & $\mathrm{C} 11$ & $120.8(2)$ \\
$\mathrm{C} 6$ & $\mathrm{C} 1$ & $\mathrm{C} 10$ & $117.9(2)$ & $\mathrm{C} 12$ & $\mathrm{C} 13$ & $\mathrm{C} 14$ & $120.0(2)$ \\
$\mathrm{O} 2$ & $\mathrm{C} 2$ & $\mathrm{C} 1$ & $122.81(19)$ & $\mathrm{C} 15$ & $\mathrm{C} 14$ & $\mathrm{C} 13$ & $119.6(2)$ \\
$\mathrm{O} 2$ & $\mathrm{C} 2$ & $\mathrm{C} 3$ & $117.2(2)$ & $\mathrm{C} 14$ & $\mathrm{C} 15$ & $\mathrm{C} 16$ & $120.4(2)$ \\
$\mathrm{C} 3$ & $\mathrm{C} 2$ & $\mathrm{C} 1$ & $120.0(2)$ & $\mathrm{C} 11$ & $\mathrm{C} 16$ & $\mathrm{C} 15$ & $120.1(2)$ \\
$\mathrm{C} 4$ & $\mathrm{C} 3$ & $\mathrm{C} 2$ & $120.3(3)$ & $\mathrm{O} 1$ & $\mathrm{C} 20$ & $\mathrm{C} 21$ & $109.57(17)$ \\
$\mathrm{C} 5$ & $\mathrm{C} 4$ & $\mathrm{C} 3$ & $120.4(2)$ & $\mathrm{C} 20$ & $\mathrm{C} 21$ & $\mathrm{C} 22$ & $107.08(19)$ \\
$\mathrm{C} 4$ & $\mathrm{C} 5$ & $\mathrm{C} 6$ & $119.6(3)$ & $\mathrm{C} 20$ & $\mathrm{C} 21$ & $\mathrm{C} 23$ & $110.5(2)$ \\
$\mathrm{C} 5$ & $\mathrm{C} 6$ & $\mathrm{C} 1$ & $120.9(3)$ & $\mathrm{C} 20$ & $\mathrm{C} 21$ & $\mathrm{C} 24$ & $110.15(19)$ \\
$\mathrm{O} 1$ & $\mathrm{C} 10$ & $\mathrm{C} 1$ & $107.37(17)$ & $\mathrm{C} 23$ & $\mathrm{C} 21$ & $\mathrm{C} 22$ & $109.38(19)$ \\
$\mathrm{O} 1$ & $\mathrm{C} 10$ & $\mathrm{C} 11$ & $112.58(17)$ & $\mathrm{C} 23$ & $\mathrm{C} 21$ & $\mathrm{C} 24$ & $109.9(2)$ \\
$\mathrm{C} 11$ & $\mathrm{C} 10$ & $\mathrm{C} 1$ & $112.27(17)$ & $\mathrm{C} 24$ & $\mathrm{C} 21$ & $\mathrm{C} 22$ & $109.73(19)$ \\
$\mathrm{C} 12$ & $\mathrm{C} 11$ & $\mathrm{C} 10$ & $121.4(2)$ & & & &
\end{tabular}

Table 6. Torsion Angles for $2 \mathrm{n}$.

\begin{tabular}{|c|c|c|c|}
\hline $\begin{array}{llll}\text { A } & \text { B } & \text { C } & \text { D }\end{array}$ & Angle $/^{\circ}$ & $\mathbf{A}$ & Angle $/^{\circ}$ \\
\hline $\mathrm{O} 1 \mathrm{C} 10 \mathrm{C} 11 \mathrm{C} 12$ & $-48.2(3)$ & C6 C1 C10O1 & $-150.0(2)$ \\
\hline $\mathrm{O} 1 \mathrm{C} 10 \mathrm{C} 11 \mathrm{C} 16$ & $132.9(2)$ & C6 C1 C10C11 & $85.8(2)$ \\
\hline O1 C 20 C21 C22 & $-179.72(18)$ & $\mathrm{C} 10 \mathrm{O} 1 \mathrm{C} 20 \mathrm{C} 21$ & $-178.31(18)$ \\
\hline O1 C 20 C 21 C 23 & $-60.7(2)$ & $\mathrm{C} 10 \mathrm{C} 1 \mathrm{C} 2 \mathrm{O} 2$ & $-3.0(3)$ \\
\hline $\mathrm{O} 1 \mathrm{C} 20 \mathrm{C} 21 \mathrm{C} 24$ & $61.0(3)$ & $\mathrm{C} 10 \mathrm{C} 1 \mathrm{C} 2 \mathrm{C} 3$ & $176.8(2)$ \\
\hline $\mathrm{O} 2 \mathrm{C} 2 \mathrm{C} 3 \mathrm{C} 4$ & $179.9(2)$ & $\mathrm{C} 10 \mathrm{C} 1 \mathrm{C} 6 \mathrm{C} 5$ & $-176.7(2)$ \\
\hline $\mathrm{C} 1 \mathrm{C} 2 \mathrm{C} 3 \mathrm{C} 4$ & $0.1(4)$ & C10C11 C12C13 & $-177.79(19)$ \\
\hline $\mathrm{C} 1 \mathrm{C} 10 \mathrm{C} 11 \mathrm{C} 12$ & $73.1(3)$ & $\mathrm{C} 10 \mathrm{C} 11 \mathrm{C} 16 \mathrm{C} 15$ & $177.6(2)$ \\
\hline $\mathrm{C} 1 \mathrm{C} 10 \mathrm{C} 11 \mathrm{C} 16$ & $-105.8(2)$ & C11 C12 C13 C14 & $0.0(3)$ \\
\hline $\mathrm{C} 2 \mathrm{C} 1 \mathrm{C} 6 \mathrm{C} 5$ & $1.6(3)$ & $\mathrm{C} 12 \mathrm{C} 11 \mathrm{C} 16 \mathrm{C} 15$ & $-1 \cdot 3(3)$ \\
\hline $\mathrm{C} 2 \mathrm{C} 1 \mathrm{C} 10 \mathrm{O} 1$ & $31.9(3)$ & $\mathrm{C} 12 \mathrm{C} 13 \mathrm{C} 14 \mathrm{C} 15$ & $-0.9(3)$ \\
\hline $\mathrm{C} 2 \mathrm{C} 1 \mathrm{C} 10 \mathrm{C} 11$ & $-92.4(3)$ & $\mathrm{C} 13 \mathrm{C} 14 \mathrm{C} 15 \mathrm{C} 16$ & $0.7(4)$ \\
\hline $\mathrm{C} 2 \mathrm{C} 3 \mathrm{C} 4 \mathrm{C} 5$ & $1.0(4)$ & $\mathrm{C} 14 \mathrm{C} 15 \mathrm{C} 16 \mathrm{C} 11$ & $0.4(4)$ \\
\hline $\mathrm{C} 3 \mathrm{C} 4 \mathrm{C} 5 \mathrm{C} 6$ & $-0.7(4)$ & C16C11 C12 C13 & $1.1(3)$ \\
\hline C4 C5 C6 C1 & $-0.6(4)$ & $\mathrm{C} 20 \mathrm{O} 1 \mathrm{C} 10 \mathrm{C} 1$ & $165.92(17)$ \\
\hline $\mathrm{C} 6 \mathrm{C} 1 \quad \mathrm{C} 2 \quad \mathrm{O} 2$ & $178.9(2)$ & $\mathrm{C} 20 \mathrm{O} 1 \mathrm{C} 10 \mathrm{C} 11$ & $-70.0(2)$ \\
\hline $\mathrm{C} 6 \mathrm{C} 1 \mathrm{C} 2 \mathrm{C} 3$ & $-1.3(3)$ & & \\
\hline
\end{tabular}


Table 7. Hydrogen Atom Coordinates $\left(\AA \times 10^{4}\right)$ and Isotropic Displacement Parameters $\left(\AA^{2} \times 10^{3}\right)$ for $2 n$.

\begin{tabular}{|c|c|c|c|c|}
\hline Atom & $x$ & $y$ & $z$ & $\mathbf{U}(\mathbf{e q})$ \\
\hline $\mathrm{H} 2$ & 4504 & 1766 & 1567 & 49 \\
\hline H3 & 959 & 1116 & 1964 & 49 \\
\hline H4 & -467 & 2095 & 2965 & 59 \\
\hline H5 & 571 & 3641 & 3799 & 57 \\
\hline H6 & 3070 & 4188 & 3649 & 44 \\
\hline H10 & 5273 & 4222 & 2851 & 27 \\
\hline H12 & 6058 & 853 & 2653 & 31 \\
\hline H13 & 7399 & -351 & 3587 & 34 \\
\hline H14 & 8235 & 585 & 4791 & 32 \\
\hline H15 & 7771 & 2736 & 5037 & 40 \\
\hline H16 & 6433 & 3952 & 4095 & 35 \\
\hline $\mathrm{H} 20 \mathrm{~A}$ & 7838 & 3337 & 2051 & 33 \\
\hline H20B & 7025 & 4660 & 1846 & 33 \\
\hline $\mathrm{H} 22 \mathrm{~A}$ & 8912 & 5104 & 843 & 54 \\
\hline $\mathrm{H} 22 \mathrm{~B}$ & 9241 & 4140 & 111 & 54 \\
\hline $\mathrm{H} 22 \mathrm{C}$ & 9715 & 3770 & 1018 & 54 \\
\hline $\mathrm{H} 23 \mathrm{~A}$ & 8322 & 1756 & 935 & 70 \\
\hline $\mathrm{H} 23 \mathrm{~B}$ & 7812 & 2073 & 27 & 70 \\
\hline $\mathrm{H} 23 \mathrm{C}$ & 6595 & 1752 & 705 & 70 \\
\hline $\mathrm{H} 24 \mathrm{~A}$ & 5324 & 3816 & 370 & 62 \\
\hline H24B & 6577 & 4151 & -285 & 62 \\
\hline $\mathrm{H} 24 \mathrm{C}$ & 6221 & 5130 & 435 & 62 \\
\hline
\end{tabular}

Table 8. Hydrogen Bonds for 2n.
D H A
$\mathrm{O} 2 \mathrm{H} 2 \mathrm{O} 1$
$\mathbf{d}(\mathbf{D}-\mathbf{H}) / \AA$
$\mathbf{d}(\mathbf{H}-\mathbf{A}) / \AA$
$\mathbf{d}(\mathbf{D}-\mathbf{A}) / \AA$
D-H-A/ ${ }^{\circ}$
0.84
1.86
$2.585(2)$
143.7 

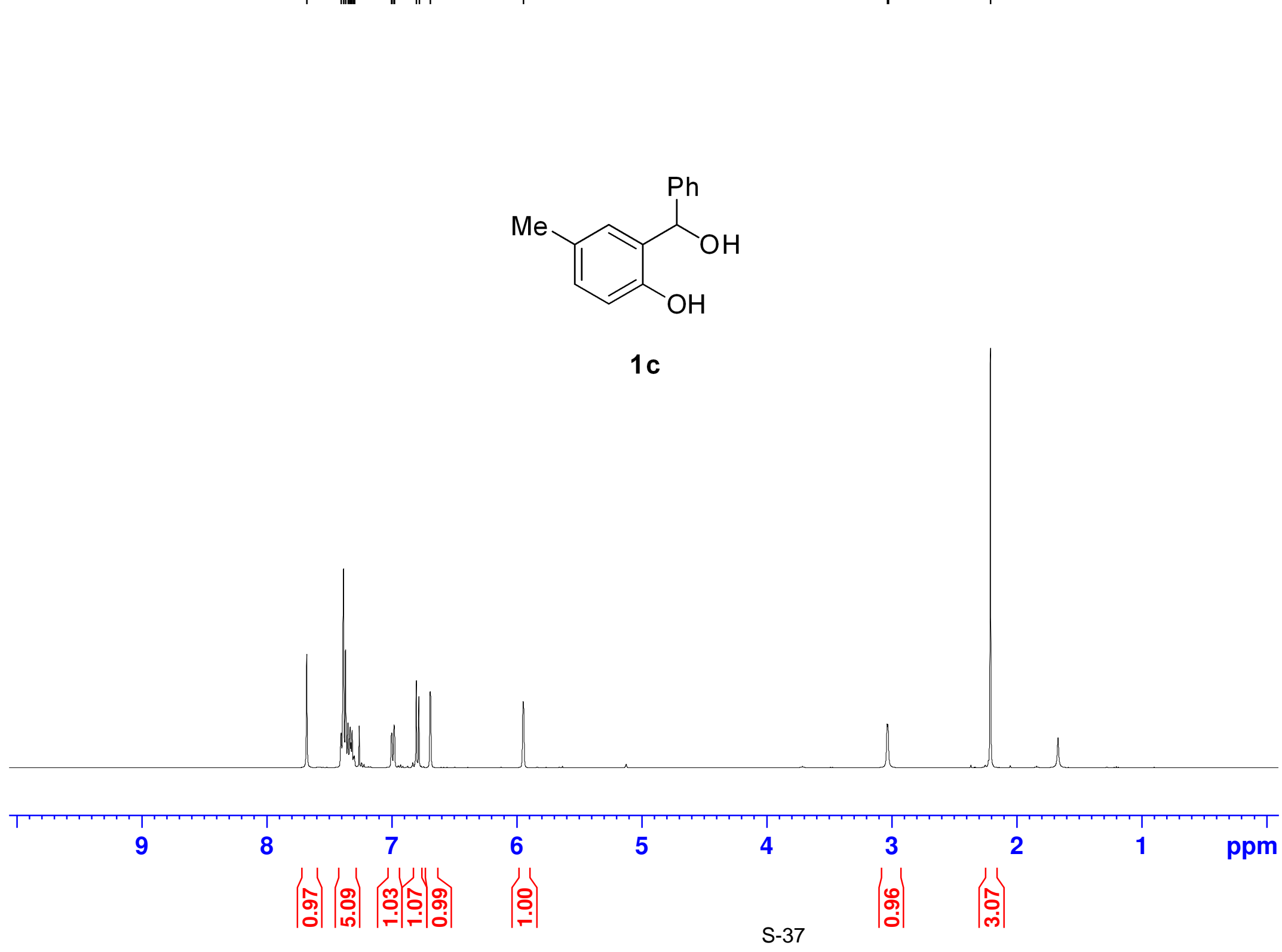

Current Data Parameters 

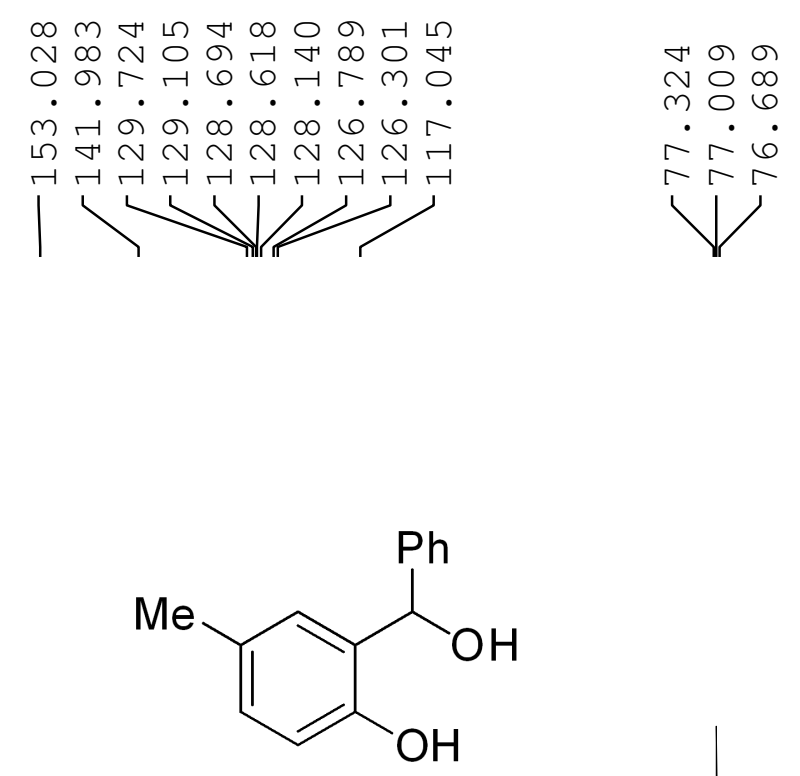

$1 \mathrm{c}$

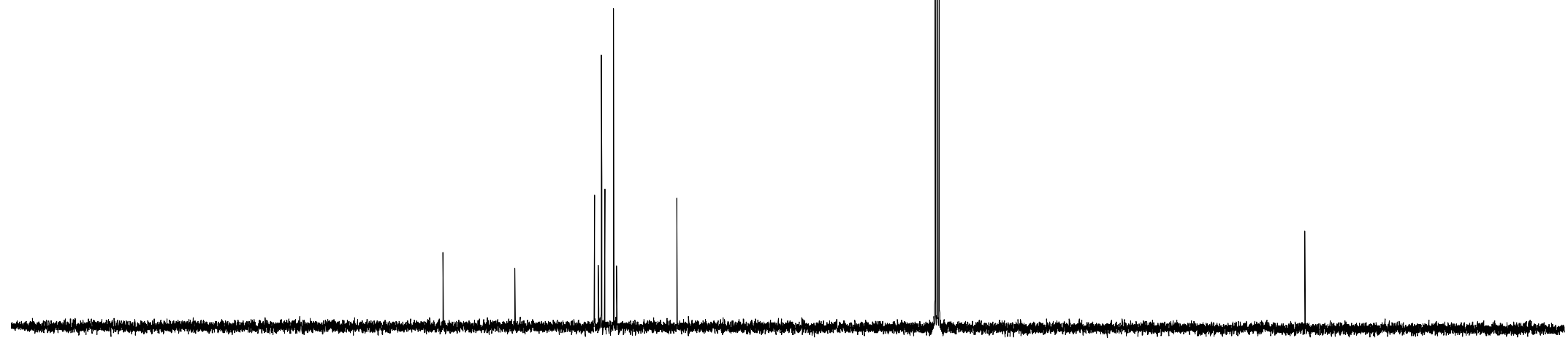

80
60

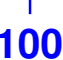

100

60

$40 \quad 20 \quad 0 \quad$ ppm

\section{Be $(1)$}

Current Data Parameters

LZW3068C-C

EXPNO

PROCNO

F2 - Acquisition Parameters

Date__ 20150627

Time 20.34

INSTRUM spect

PROBHD

zgpg30
65536

$\begin{array}{ll}\text { TD } & 65536 \\ \text { SOLVENT } & \text { CDC13 }\end{array}$

DS

SWH $24038.461 \mathrm{~Hz}$

FIDRES $\quad 0.366798 \mathrm{~Hz}$

$\mathrm{AQ} \quad 1.3631488 \mathrm{sec}$

RG

DW

$\mathrm{DE}$

D1
D11

D11
TD 0

$=======$

$\mathrm{SFO1}$

NUC

P1
PLW1

$1.3631488 \mathrm{sec}$
196.92 196.92
20.800 usec 6.50 usec $299.2 \mathrm{~K}$

$2.00000000 \mathrm{sec}$ $0.03000000 \mathrm{sec}$

CHANNEL f1 $========$ $100.6228298 \mathrm{MHz}$ $13 \mathrm{C}$

9.70 usec
$46.98899841 \mathrm{~W}$

$========$ CHANNEL $\mathrm{f} 2========$ SFO2 $400.1316005 \mathrm{MHz}$

NUC2

CPDPRG [ 2

PCPD2

PLW2

PLW12
PLW13

waltz16

90.00 usec

$11.99499989 \mathrm{~W}$

$0.34213999 \mathrm{~W}$

$0.27713001 \mathrm{~W}$

2 - Processing parameters SI 32768

SF $\quad 100.6127725 \mathrm{MHz}$

$\begin{array}{llc}\text { WDW } & & \text { EM } \\ \text { SSB } & 0 & 1.00 \mathrm{~Hz} \\ \text { LB } & 0 & \end{array}$

$\begin{array}{lll}\mathrm{GB} & 0 & 1.40\end{array}$ 


\section{aruxer $(1)$}
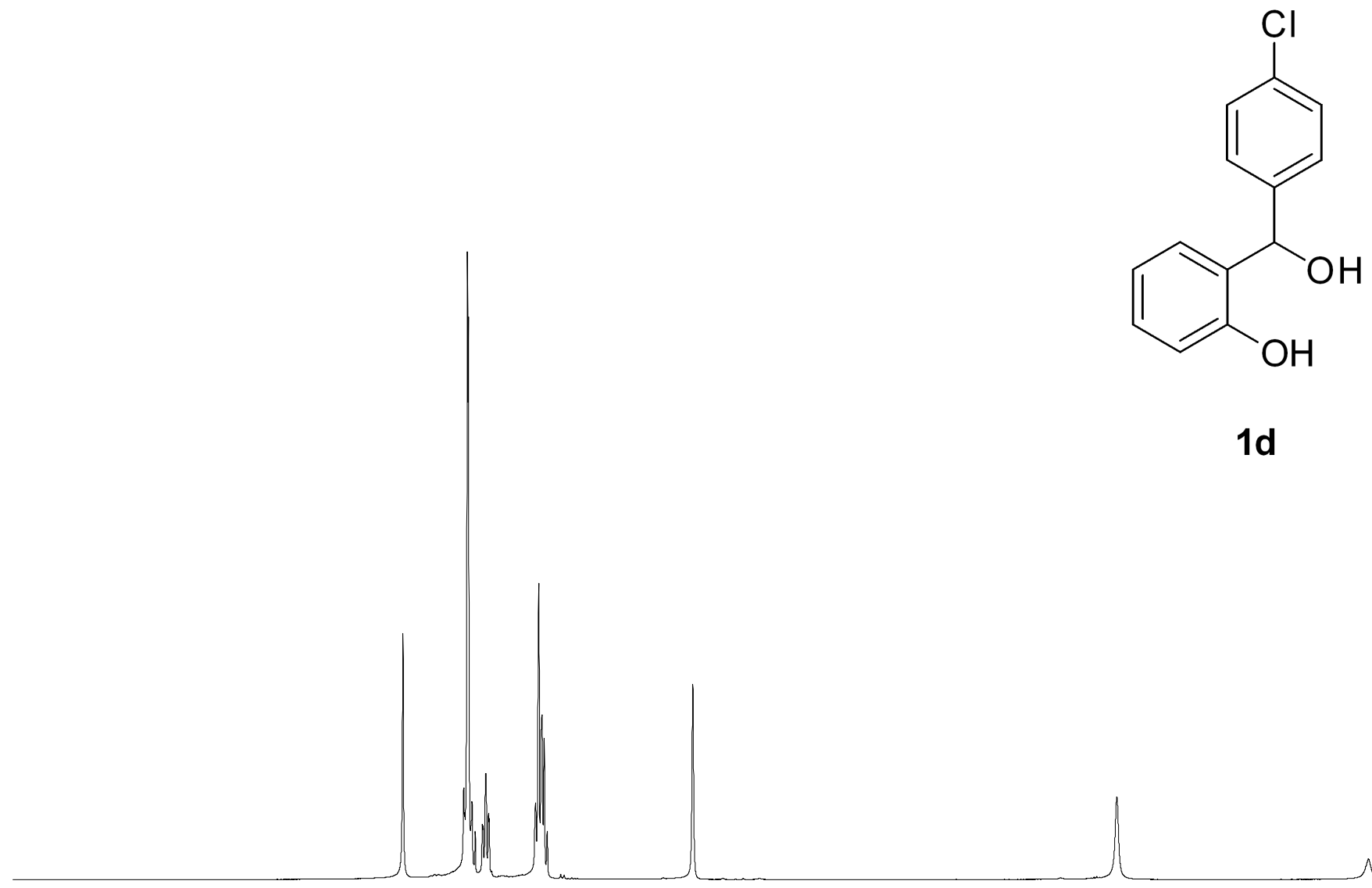

$1 d$

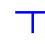

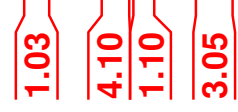

6

5

임

3

2

$1 \quad \mathrm{ppm}$

Current Data Parameters

PROCNO

lzw3068D-H

F2 - Acquisition Parameters

Date_ 20150627

$\begin{array}{lr}\text { Time } & 22.17\end{array}$

INSTRUM spect

ROBHD

Zg30
PULPROG

$\begin{array}{ll}\text { TD } & 65536 \\ \text { SOLVENT } & \end{array}$

SOLVENT

NS

DS

FID

FIDRES
AQ
RG

DW

$\mathrm{DE}$

$\mathrm{TE}$

D1
TD 0

16

$8012.820 \mathrm{~Hz}$

$0.122266 \mathrm{~Hz}$

$4.0894465 \mathrm{sec}$ 49.32

62.400 usec 6.50 usec $1.00000000 \mathrm{sec}$

$=======$ CHANNET $\mathrm{fl}=======$ SFO1 NUC1

$\mathrm{NUC}$
$\mathrm{P} 1$

CHANNEL $\mathrm{f} 1==$

$400.1324710 \mathrm{MHz}$

PLW1

14.50 usec

F2 - Processing parameters

SF

SF
WDW

$400.1300089 \mathrm{MHz}$

SSB

EM

$0.30 \mathrm{~Hz}$

GB
PC

1.00 


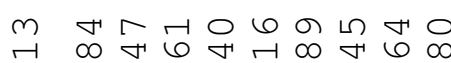

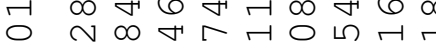

$\dot{0} \dot{\circ} \dot{\sigma} \dot{\infty} \dot{\infty} \dot{0} \dot{\circ}$

レ

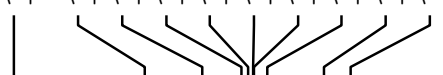

1

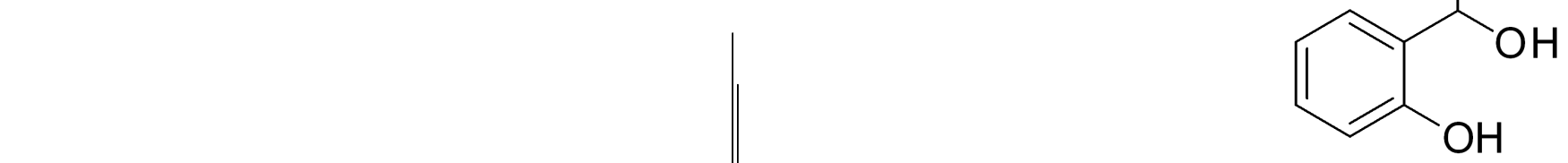

1d<smiles>Oc1ccccc1C(O)c1ccc(Cl)cc1</smiles>

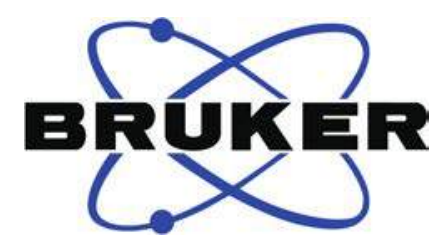

Current Data Parameters NAME LZW3068D-C

PROCNO

F2 - Acquisition Parameters

Date_ 20150627

Time $\quad 20.26$

$5 \mathrm{~mm}$ spect

zgpg30

TD

NS

NS

SWH

FIDRES

FIDR
AQ
RG

DW

DE

TE

D1
D11

TD 0

$=====$

$\mathrm{SFO1}$

$\mathrm{NUC1}$
$\mathrm{P} 1$

P 1
PLW1

CDC13

52

$24038.461 \mathrm{~Hz}$

$1.3631488 \mathrm{~Hz}$

196.92

20.800 usec 6.50 usec

$299.1 \mathrm{~K}$

$2.00000000 \mathrm{sec}$ $0.03000000 \mathrm{sec}$

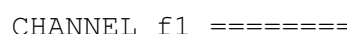
$100.6228298 \mathrm{MHz}$ $13 \mathrm{C}$

$\begin{aligned} & 9.70 \text { usec } \\ & 46.98899841 \mathrm{~W}\end{aligned}$

$========$ CHANNEL $\mathrm{f} 2========$ SEO2

NUC2

CPDPRG [2

CPD 2

PLW2

PLW12

PLW13

$400.1316005 \mathrm{MHz}$

$1 \mathrm{H}$

90.00 usec

$11.99499989 \mathrm{~W}$

$0.34213999 \mathrm{~W}$

$0.27713001 \mathrm{~W}$

F2 - Processing parameters

SI $\quad 32768$

$100.6127751 \mathrm{MHz}$

ppm

SS

$1.00 \mathrm{~Hz}$

1.40 


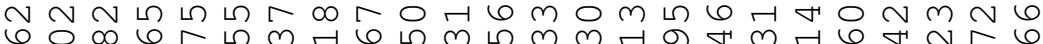
$\infty \quad 0$ ம

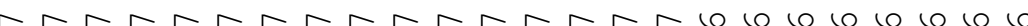
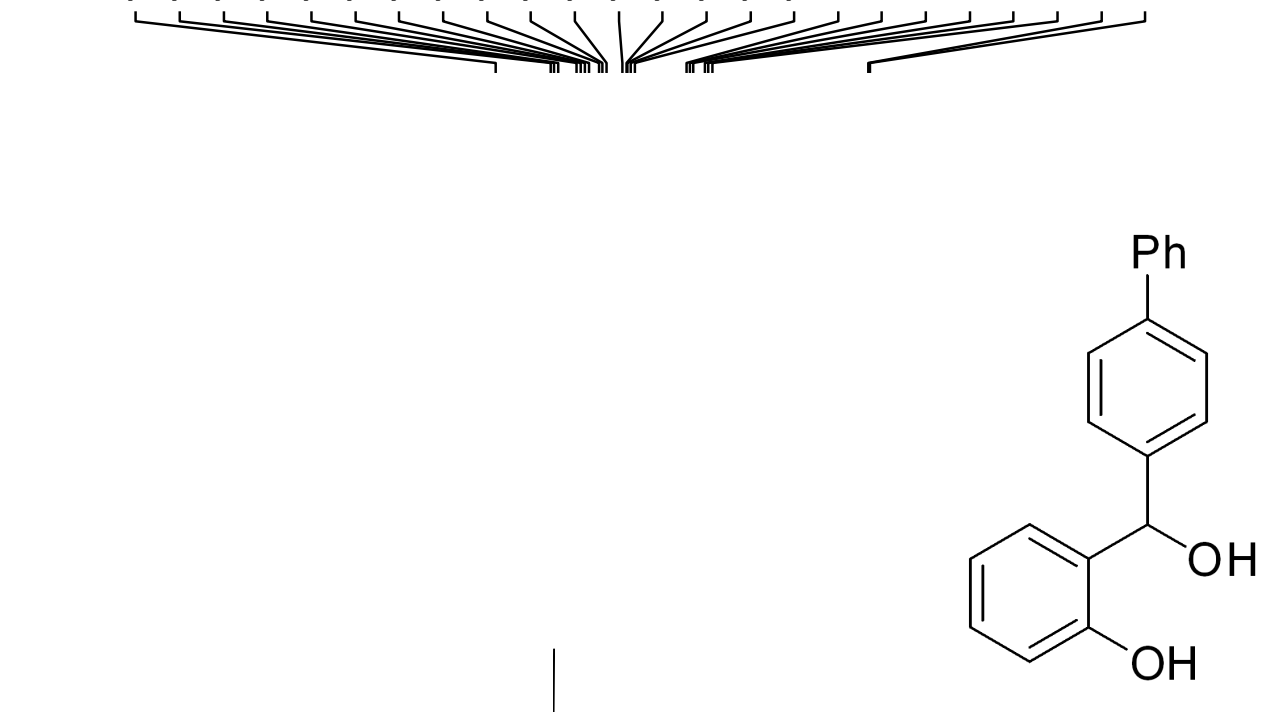

1e

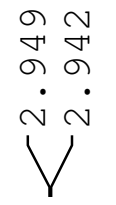

\section{en $(1>$}

Current Data Parameters NAME

EXPNO

lzw3068E-H

PROCNO

F2 - Acquisition Parameters

Date__ 20150627

Time 22.20

$5 \mathrm{~mm}$ spect

TISOC

$\mathrm{zg} 30$
65536

$\begin{array}{ll}\text { TD } & 65536 \\ \text { SOLVENT } & \text { CDC13 }\end{array}$

NS

DS

FIDRES

$\mathrm{AQ}$

$\mathrm{RG}$

$\mathrm{DW}$

$\mathrm{DE}$

TE

TD 0

$===$

$\mathrm{SFO1}$

$\mathrm{NUC}$

$\mathrm{CDCl} 3$
8
0

$8012.820 \mathrm{~Hz}$

$0.122266 \mathrm{~Hz}$

$4.0894465 \mathrm{sec}$ 187.77

62.400 usec 6.50 usec $1.00000000 \mathrm{sec}$

CHANNEL $\mathrm{f} 1========$ $400.1324710 \mathrm{MHz}$

- Processing parameter

SI $\quad 65536$

SF $\quad 400.1300111 \mathrm{MHz}$

WSW

EM

$0.30 \mathrm{~Hz}$

1.00

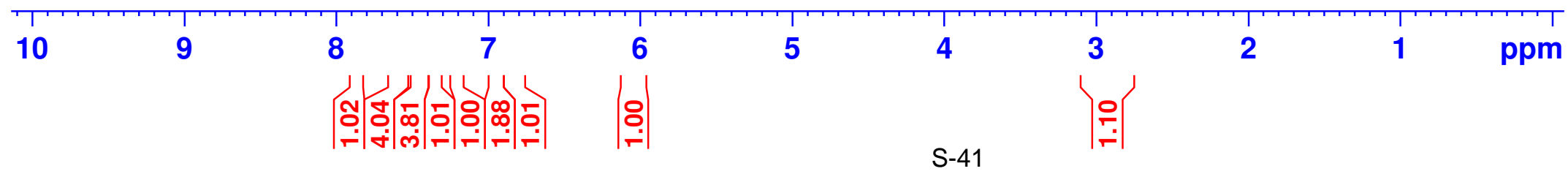




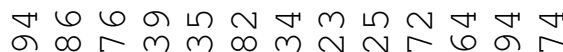

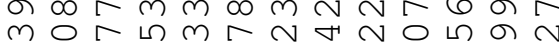

ம் $\dot{0} \dot{0} \dot{0} \dot{\infty} \dot{\sim} \dot{\sim} \dot{0} \dot{\circ}$

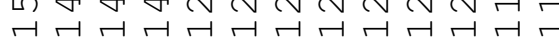

$\longrightarrow 3$

mor

$\therefore \sim \dot{6}$

4<smiles>Oc1ccccc1C(O)c1ccc(-c2ccccc2)cc1</smiles>

$1 e$

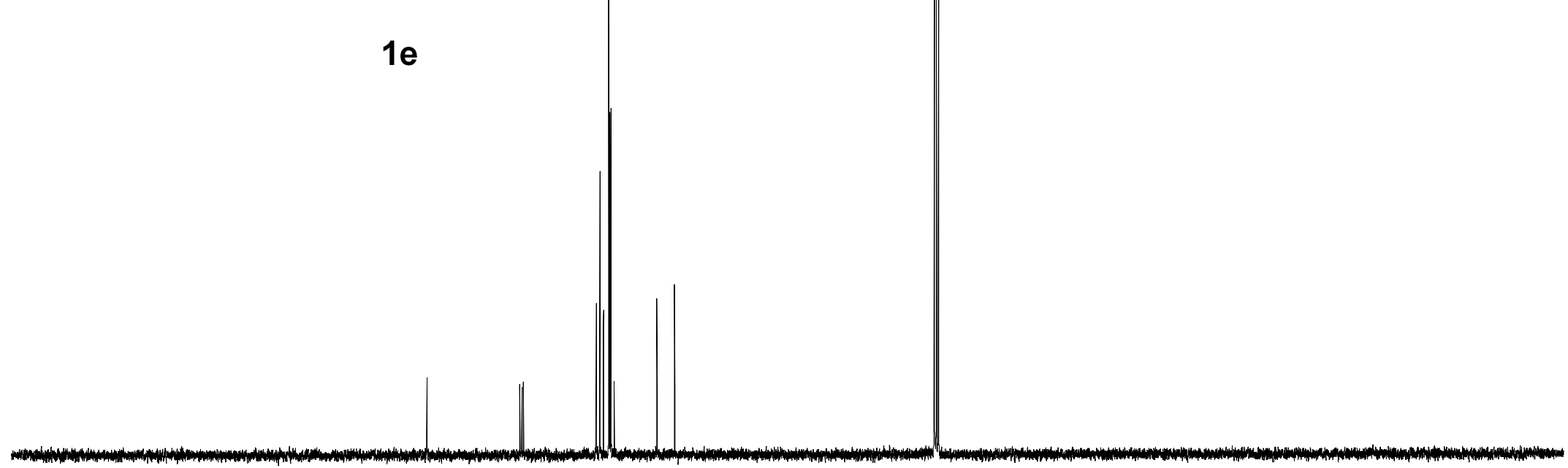

80

60 


\section{sperer $(1)$}

Current Data Parameters NAME

EXPNO

LZW30 68F-H<smiles>CSc1ccc(C(O)c2ccccc2O)cc1</smiles>
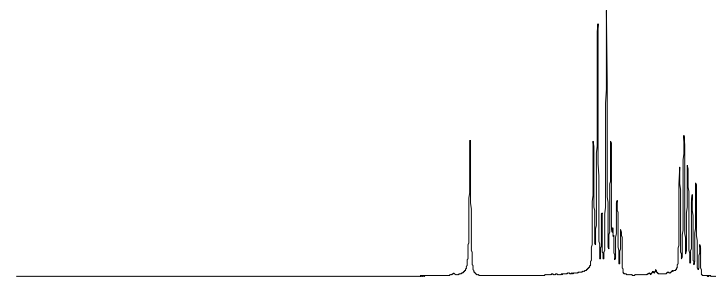

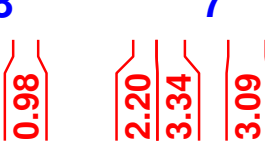

6

5

4
2

|
F2 - Acquisition Parameters

Date_ 20150627

$\begin{array}{lr}\text { Time } & 19.57\end{array}$

INSTRUM spect

PROBHD $5 \mathrm{~mm}$ PABBO BB/

PULPROG $\quad$ zg30

$\begin{array}{lr}\text { TD } & \text { Zg30 } \\ & 65536\end{array}$

$\begin{array}{ll}\text { TD } & 65536 \\ \text { SOLVENT } & \text { CDCl3 }\end{array}$

$\begin{array}{lr}\text { NS } & 14\end{array}$

DS

SWH $\quad 8012.820 \mathrm{~Hz}$

FIDRES $\quad 0.122266 \mathrm{~Hz}$

$\begin{array}{cr}\mathrm{AQ} & 4.0894465 \mathrm{sec} \\ \mathrm{RG} & 82.92\end{array}$

$\begin{array}{lr}\text { RG } & 82.92 \\ \text { DW } & 62.400 \text { usec }\end{array}$

$\begin{array}{lr}\text { DW } & 62.400 \text { usec } \\ \text { DE } & 6.50 \text { usec }\end{array}$

$298.1 \mathrm{~K}$

$\begin{array}{lr}\text { D1 } & 1.0000000 \\ \text { TD } & 1\end{array}$

$=======$ CHANNEL $\mathrm{f} 1 \quad========$

SFO1 $\quad 400.1324710 \mathrm{MHz}$

NUC1 $1 \mathrm{H}$

P1 14.50 usec

PLW1 $11.99499989 \mathrm{~W}$

F2 - Processing parameters

SI 65536

SF $\quad 400.1300088 \mathrm{MHz}$

WDW EM

$0.30 \mathrm{~Hz}$

$\mathrm{GB}$
$\mathrm{PC}$

1.00 

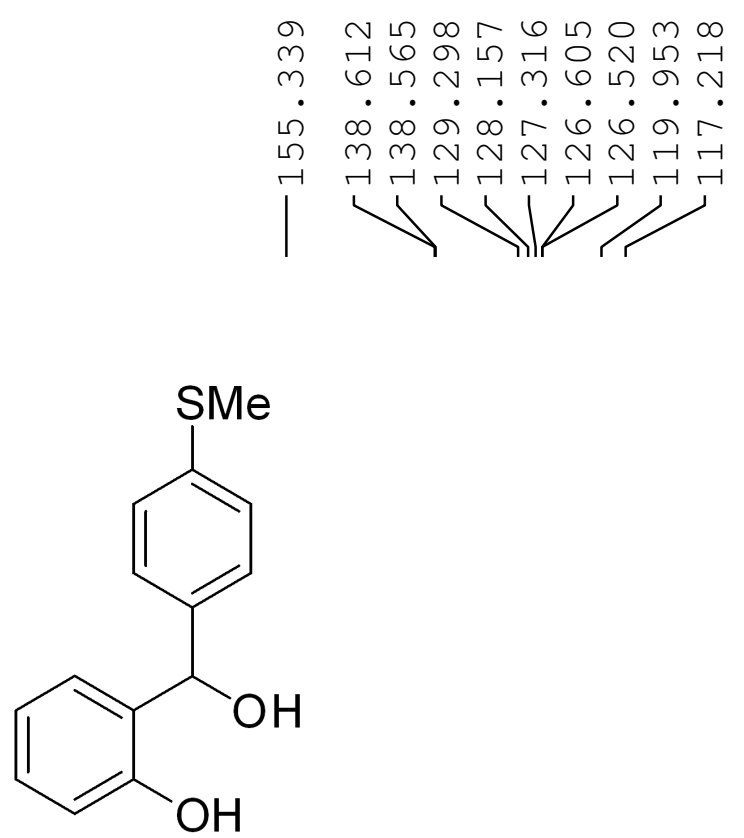

$1 f$

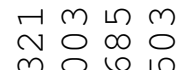

$\therefore \dot{a} \dot{0}$

든

$W$

\section{BR $(1)$}

Current Data Parameters

NAME

LZW3068F-C

PROCNO

F2 - Acquisition Parameter

Date_ 20150627

20.01

Time

PROBHD

PULPROG zgpg30

$5 \mathrm{~mm}$ PABBO BB/

SOLVENT

NS

NS
DS

SWH

FIDRES

$\mathrm{AQ}$

RG

DW

DE

D1
D11
TD0

$======$

$\mathrm{SFO1}$

NUC

P 1
PLW1

zgpg 30
65536

$\mathrm{CDCl} 13$ 44

$24038.461 \mathrm{~Hz}$ $24038.461 \mathrm{~Hz}$ $0.366798 \mathrm{~Hz}$ 196.92 196.92
20.800 usec 6.50 usec $298.9 \mathrm{~K}$

$2.00000000 \mathrm{sec}$ $0.03000000 \mathrm{sec}$ .03000000
1

CHANNEL $f 1=======$ $100.6228298 \mathrm{MHz}$ $13 \mathrm{C}$ 9.70 usec
$46.98899841 \mathrm{~W}$

$========$ CHANNEL $\mathrm{f} 2=======$ SEO2

NUC2

CPDPRG [2

PCPD2

PLW2

PLW13

$400.1316005 \mathrm{MHz}$ $1 \mathrm{H}$

waltz16 11.99499989 usec 0.34213999 $0.27713001 \mathrm{~W}$

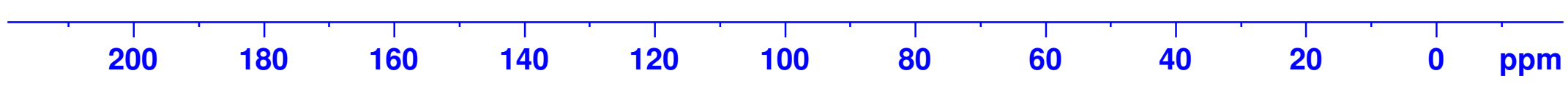

2 - Processing parameters SI 32768

(2)
100

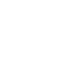

60

40 
অm

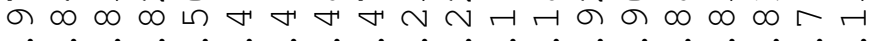

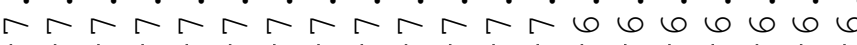

$\ldots$
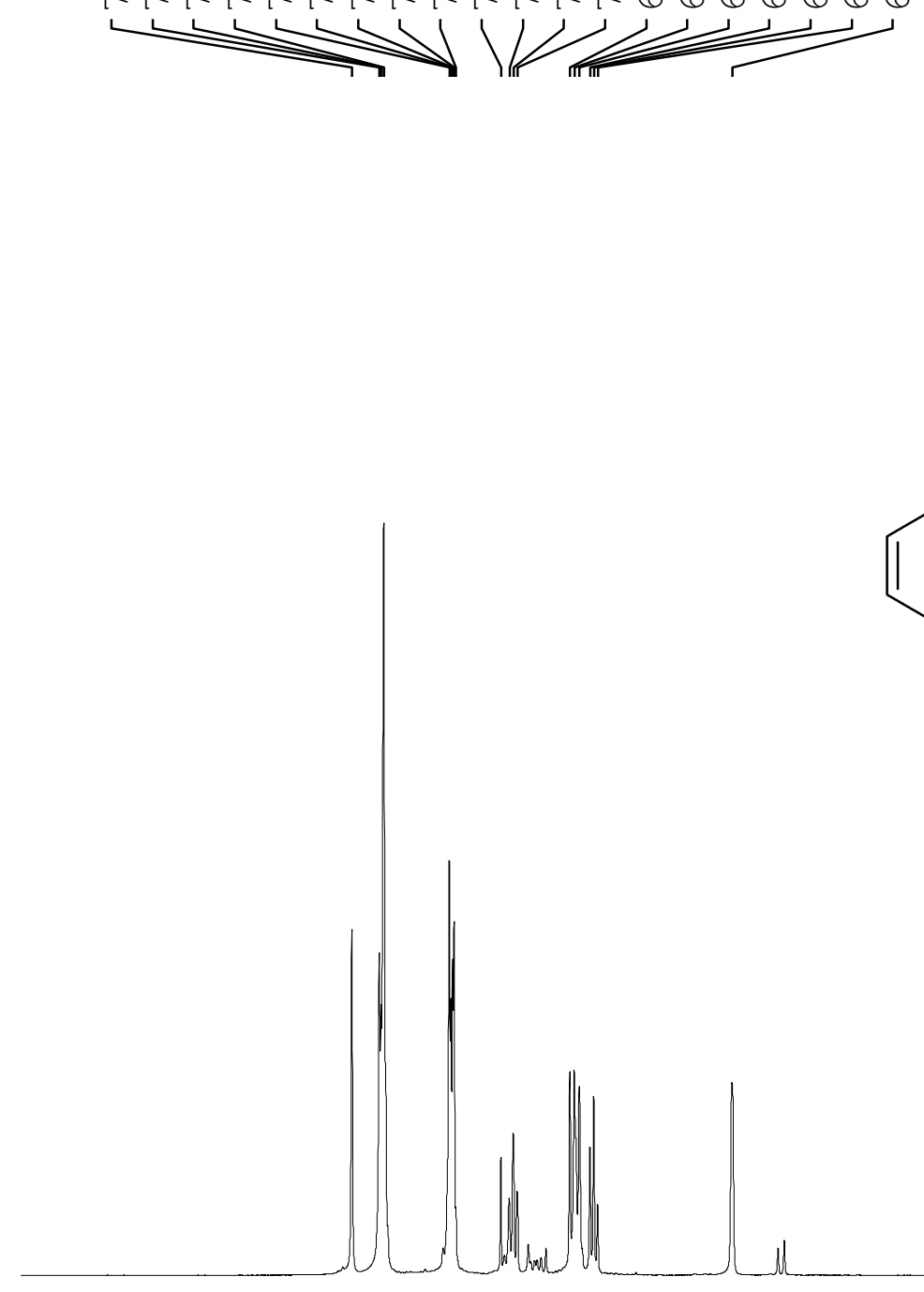

ก

$\sim \sim$

$\dot{m} \dot{m}$

Vin

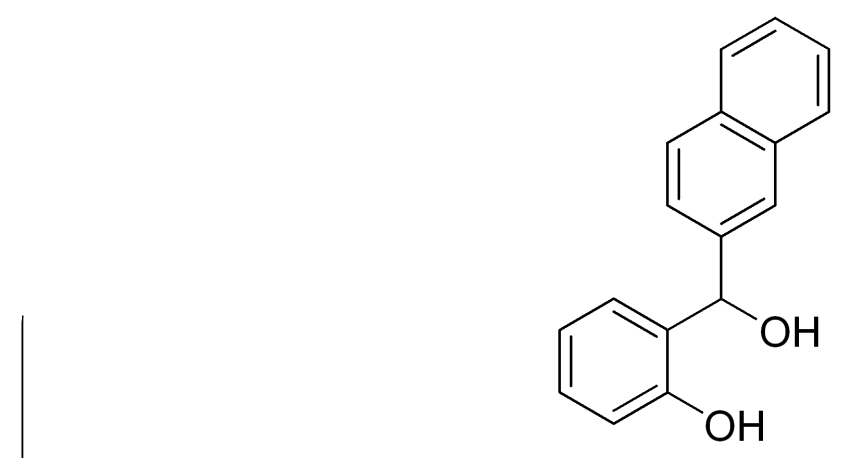

$1 \mathrm{~g}$

\section{BpUKER

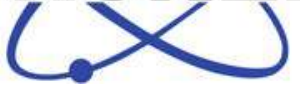

Current Data Parameters

$$
\begin{aligned}
& \text { NAME } \\
& \text { EXPNO }
\end{aligned}
$$

PROCNO

$1 \mathrm{zW} 3068 \mathrm{~A}-\mathrm{H}$

F2 - Acquisition Parameters

Date__ 20150627

$\begin{array}{lr}\text { Time } & 22.08\end{array}$

INSTRUM spect

zg30
65536

TD $\quad 65536$

SOLVENT

NS

DS

FIDRES

FIDR
AQ
RG

$\mathrm{RG}$

DW
DE
TE

TE

TD 0

$===$

$\mathrm{SFO1}$

$\mathrm{NUC}$
$\mathrm{P} 1$

P1
PLW1

$\mathrm{CDC} 13$
10

$8012.820 \mathrm{~Hz}$

$4.0894465 \mathrm{~Hz}$

70.97

62.400 usec

6.50 usec

$1.00000000 \mathrm{sec}$

f1 $1===$

$400.1324710 \mathrm{MHz}$ 14.50

4.50 use

$11.99499989 \mathrm{~W}$

F2 - Processing parameters

SI 65536

$\begin{array}{ll}\text { SF } & 400.1300098 \mathrm{MHz}\end{array}$

WDW

SSB

EM

$0.30 \mathrm{~Hz}$

1.00

$\begin{array}{lllllllllllllllllll}9.0 & 8.5 & 8.0 & 7.5 & 7.0 & 6.5 & 6.0 & 5.5 & 5.0 & 4.5 & 4.0 & 3.5 & 3.0 & 2.5 & 2.0 & 1.5 & 1.0 & \text { ppm }\end{array}$

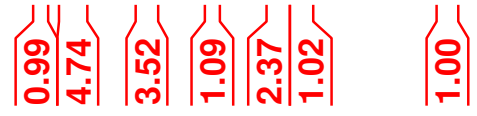




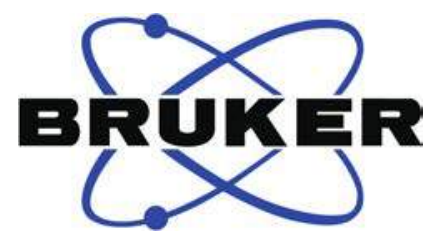

Current Data Parameters NAME lzw3068A-C

PROCNO

F2 - Acquisition Parameters Date_ 20150627

$\begin{array}{lr}\text { Time } & 22.06\end{array}$

SPect

zgpg30

TD $\quad 65536$

SOLVENT

NS

SWH

FIDRES

FIDR
AQ

AQ

$\mathrm{DW}$

DE

$1 \mathrm{~g}$

D1

D 11

$======$

$\mathrm{SFO1}$

NUC

P1

PLW1

$\mathrm{CDCl} 3$

103

$24038.461 \mathrm{~Hz}$ $0.366798 \mathrm{~Hz}$ $1.3631488 \mathrm{sec}$ 196.92 20.800 usec 6.50 usec $299.4 \mathrm{~K}$ $2.00000000 \mathrm{sec}$ 0.03000000

CHANNET 61 = $===$ $100.6228298 \mathrm{MHz}$ $13 \mathrm{C}$ $46.98899841 \mathrm{~W}$

$=======$ CHANNEL $\mathrm{f} 2========$ SEO2

$$
\text { NUC2 }
$$

CPDPRG [2

PCPD2

PLW2

PLW12

PLW13

$400.1316005 \mathrm{MHz}$ $\begin{array}{r}1 \mathrm{H} \\ \hline\end{array}$

$$
\text { waltz16 }
$$
90.00 usec $11.99499989 \mathrm{~W}$ $0.34213999 \mathrm{~W}$ $0.27713001 \mathrm{~W}$

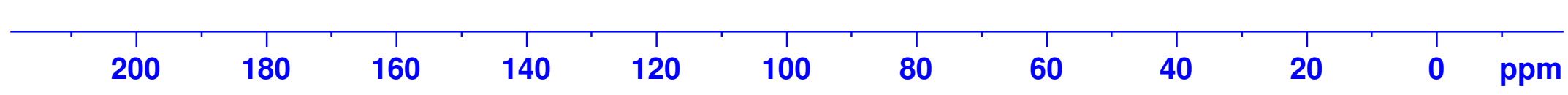

F2 - Processing parameters SI 32768 SF $\quad 100.6127738 \mathrm{MHz}$ 


\section{BR X $(1)$}

Current Data Parameters NAME

EXPNO

LZW3068G-H

PROCNO<smiles>COc1ccccc1C(O)c1ccccc1O</smiles>

2 - Acquisition Parameter

Date__ 20150627

Time 20.05

INSTRUM spect

ROBHD

PULPROG $\quad$ zg30

TD $\quad 65536$

SOLVENT

NS

DS

$\begin{array}{ll}\text { SWH } & 8012.820 \mathrm{~Hz} \\ \text { FIDRES } & 0.122266 \mathrm{~Hz}\end{array}$ $4.122266 \mathrm{~Hz}$

DW

$1 \mathrm{~h}$

DE

TE

$0894465 \mathrm{sec}$ 62.93

62.400 usec 6.50 usec
$298.1 \mathrm{~K}$ $1.00000000 \mathrm{sec}$

TD 0

$===$

1.00000000

$1 \mathrm{sec}$

$\mathrm{SFO1}$

NUC

CHANNEL $\mathrm{fl}========$

$400.1324710 \mathrm{MHz}$

PLW1

14.50 usec

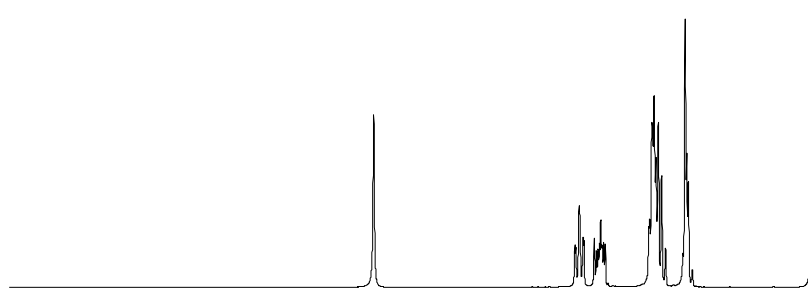

Processing parameters

SI $\quad 65536$

SF $\quad 400.1300000 \mathrm{MHz}$

WDW

EM

$0.30 \mathrm{~Hz}$

$\mathrm{GB}$

1.00

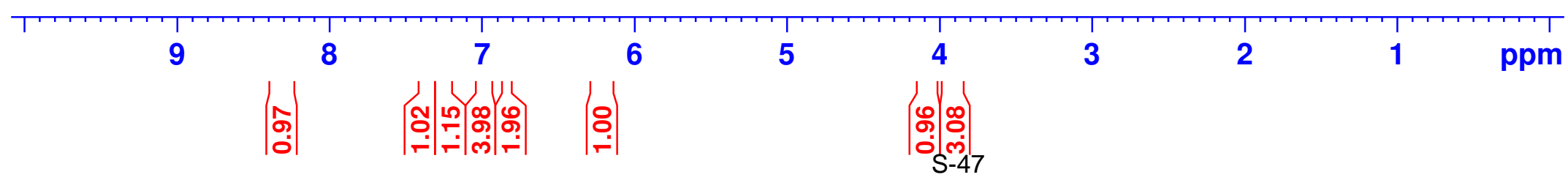




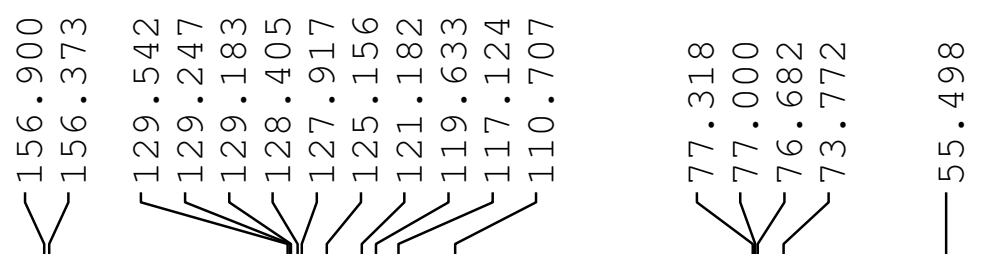

\section{Be $(1)$}

Current Data Parameters NAME

PROCNO

LZW3068G-C<smiles>COc1ccccc1C(O)c1ccccc1O</smiles>

$1 \mathrm{~h}$

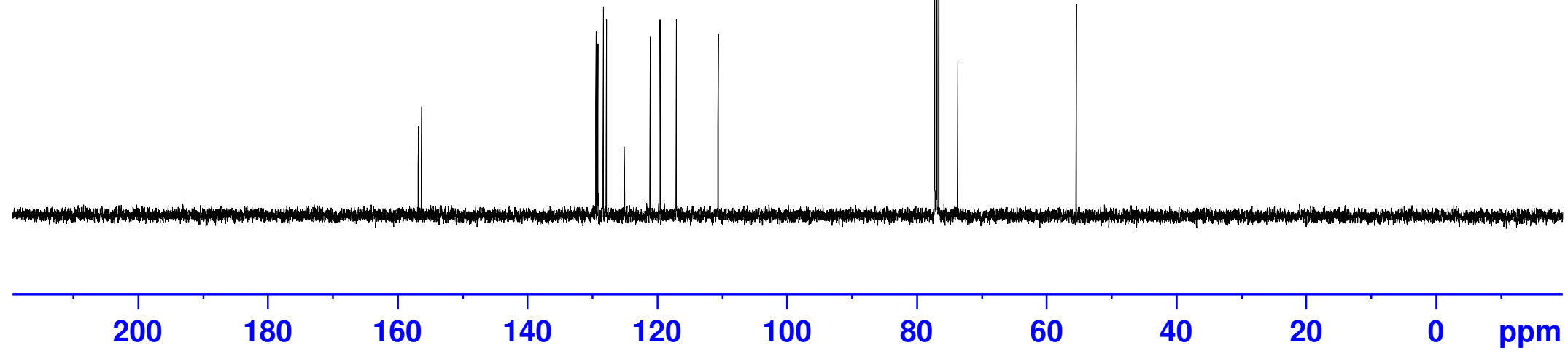

F2 - Acquisition Parameters

Date_ 20150627

PULPROG

SOLVENT
CDCl3

DS

SWH

FIDRES

$\mathrm{AQ}$

RG

DW
DE
TE
D1

D1

D 11

(1D0

$\mathrm{SFO} 1$

NUC1

$\mathrm{P} 1$

PLW1

$24038.461 \mathrm{~Hz}$

$24038.461 \mathrm{~Hz}$

$1.3631488 \mathrm{sec}$

196.92

20.800 usec

6.50 usec

$298.9 \mathrm{~K}$

$2.00000000 \mathrm{sec}$ $0.03000000 \mathrm{sec}$

CHANNET $\mathrm{f1}=$ $100.6228298 \mathrm{MHz}$ $13 \mathrm{C}$

$\begin{aligned} & 9.70 \text { usec } \\ & 46.98899841 \mathrm{~W}\end{aligned}$

$=======$ CHANNEL $\mathrm{f} 2========$ SEO

NUC2

CPDPRG [2

PCPD2

LW2

PLW12

PLW13

$400.1316005 \mathrm{MHz}$

$1 \mathrm{H}$

90.00 usec

$11.99499989 \mathrm{~W}$

$0.34213999 \mathrm{~W}$

2 - Processing parameters

SI $\quad 32768$

WDW $\quad 100.6127750 \mathrm{MHz}$

LB
GB
PC

$\triangle B$
$G B$
$P C$

$1.00 \mathrm{~Hz}$

1.40
Time $\quad 20.10$

$5 \mathrm{~mm}$ spect

zgpg30
65536 
ค

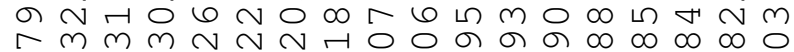

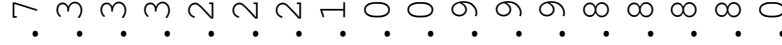
rararrara $60666_{6} 66_{6}$

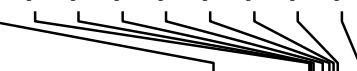
/

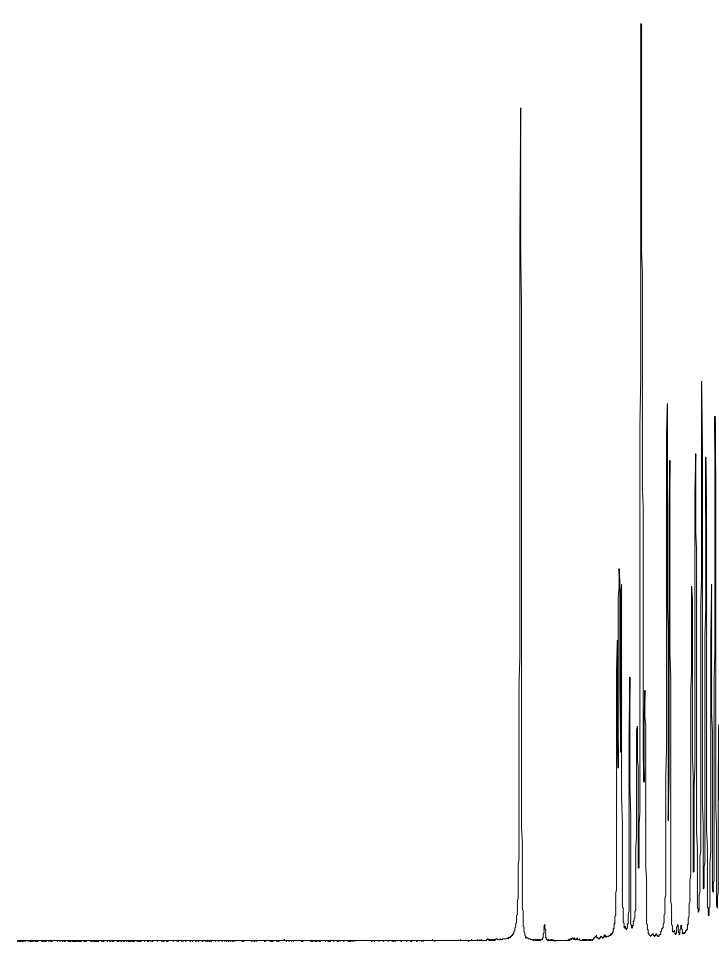

\section{ex $(1)$}

Current Data Parameters NAME

EXPNO
PROCNO

LZW3068H-H

2 - Acquisition Parameters

Date__ 20150627

Time 20.14

$5 \mathrm{~mm}$ spect

\begin{tabular}{rr} 
PULPROG & zg30 \\
\hline
\end{tabular}

SOLVENT CDCl3

DS

$1 \mathrm{i}$

SWH

FIDRES

$0.122266 \mathrm{~Hz}$

103.52

62.400 usec 6.50 usec

DW

$\mathrm{DE}$

$\mathrm{TE}$
$\mathrm{D} 1$

TD 0

$$
1
$$

$=======$ CHANNEI $\mathrm{f} 1========$ SFO1 $400.1324710 \mathrm{MHz}$ NUC1

P1 14.50 usec PLW1 $11.99499989 \mathrm{~W}$

F2 - Processing parameters

SI 65536

SF $\quad 400.1300092 \mathrm{MHz}$

WDW

$S S B$

EM

$0.30 \mathrm{~Hz}$

1.00 


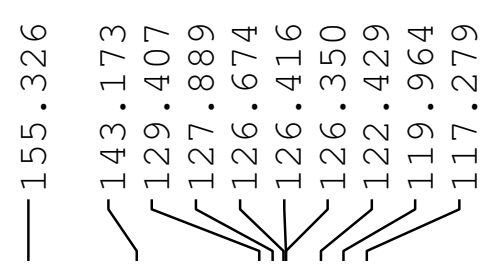

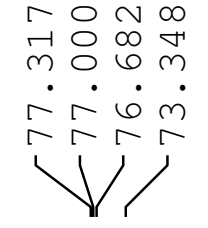

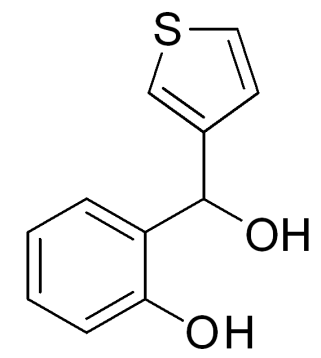

$1 \mathrm{i}$
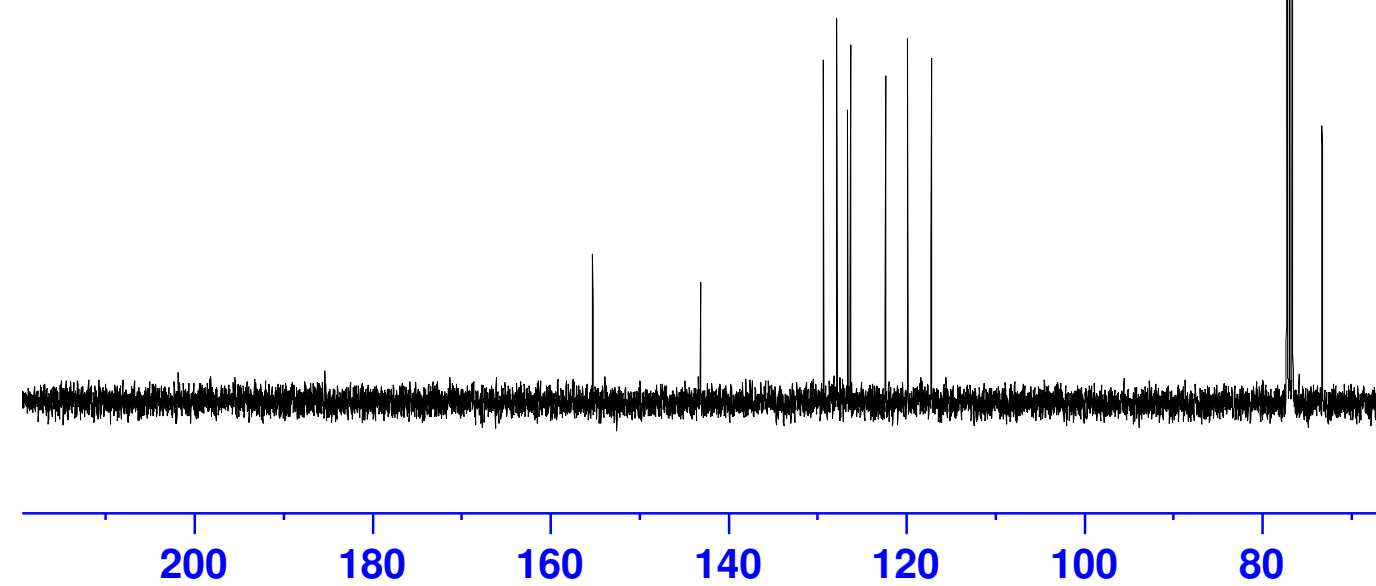

180

160

140

120

80

60

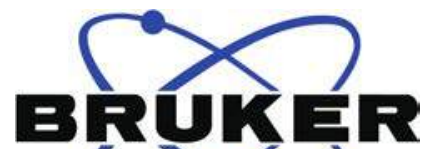 $(0<)$}

Current Data Parameters

$$
\begin{aligned}
& \text { NAME } \\
& \text { EXPNO }
\end{aligned}
$$

PROCNO

F2 - Acquisition Parameters

Date__ 20150627

$\begin{array}{lr}\text { Time- } & 20.18 \\ \text { TNSTRUM } & \end{array}$

INSTRUM Spect

PROBHD

zgpg30
TD

$\begin{array}{lr}\text { TD } & 65536 \\ \text { SOLVENT } & \text { CDCl3 } \\ \text { NS } & 50\end{array}$

DS

SWH

FIDRES

$\mathrm{AQ}$
$\mathrm{RG}$
$\mathrm{DW}$

$\mathrm{DW}$
$\mathrm{DE}$

DE

D1
D11

D11

$=====$

$\mathrm{SFO1}$
$\mathrm{NUC1}$

$\mathrm{NUC1}$
$\mathrm{P} 1$

P1
P LW1

0
$24038.461 \mathrm{~Hz}$
$0.366798 \mathrm{~Hz}$

$\begin{aligned} 1.3631488 \mathrm{sec} & \end{aligned}$

196.92

20.800 usec

6.50 usec

$2.00000000 \mathrm{~K}$

$2.00000000 \mathrm{sec}$
$0.03000000 \mathrm{sec}$

CHANNEL $\mathrm{fl}========$

$100.6228298 \mathrm{MHz}$ $13 \mathrm{C}$

9.70 usec
$46.98899841 \mathrm{~W}$

$========$ CHANNEL $f 2========$

$\mathrm{SFO} 2$

NUC2

CPDPRG [2

PCPD2

PLW2

PLW12

PLW13

$400.1316005 \mathrm{MHz}$

$1 \mathrm{H}$

90.00 usec

$11.99499989 \mathrm{~W}$

$0.34213999 \mathrm{~W}$
$0.27713001 \mathrm{~W}$

F2 - Processing parameters

SI $\quad 32768$

$\begin{array}{lc}\text { SF } & 100.6127733 \mathrm{MHz} \\ \text { WDW } & \mathrm{EM}\end{array}$

SSB

$1.00 \mathrm{~Hz}$

1.40 
の $N$ の

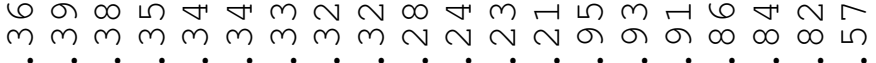

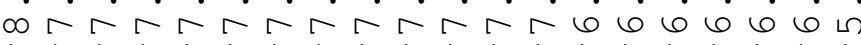

$\longrightarrow$

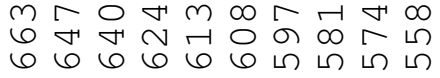
$\dot{m} \dot{m} \dot{m} \dot{m} \dot{m} \dot{m} \dot{m} \dot{m} \dot{m}$ $\longrightarrow$

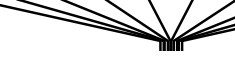<smiles>CCCCOC(c1ccccc1)c1ccccc1O</smiles>

$2 a$

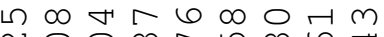

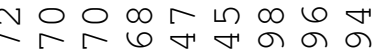
$31 /$

\section{BR $(1)$}

Current Data Parameters NAME

PROCNO

lzw2102F-H

F2 - Acquisition Parameters

Date__ 20150401

Time $\quad 13.15$

$5 \mathrm{mmect}$

PULPROG $\quad$ zg30

$\begin{array}{lr}\text { TD } & \text { zg30 } \\ & 65536\end{array}$

$\begin{array}{ll}\text { SOLVENT } & 65536 \\ \text { CDC13 }\end{array}$

DS

DWH

FIDRES

AQ

RG

DW

$\mathrm{DE}$

D1

TD 0

$===$

$\mathrm{SFO1}$

$\mathrm{NUC}$

$\mathrm{CDC}$
16

$8012.820 \mathrm{~Hz}$

$0.122266 \mathrm{~Hz}$

$4.0894465 \mathrm{sec}$ 49.32

62.400 usec 6.50 usec

$1.00000000 \mathrm{sec}$

CHANNET $\mathrm{f} 1=$ $400.1324710 \mathrm{MHz}$ 400.1324710 .99499989 W

F2 - Processing parameters

SI $\quad 65536$

$\begin{array}{ll}\text { SF } & 400.1300000 \mathrm{MHz}\end{array}$

WDW

EM

$0.30 \mathrm{~Hz}$

1.00

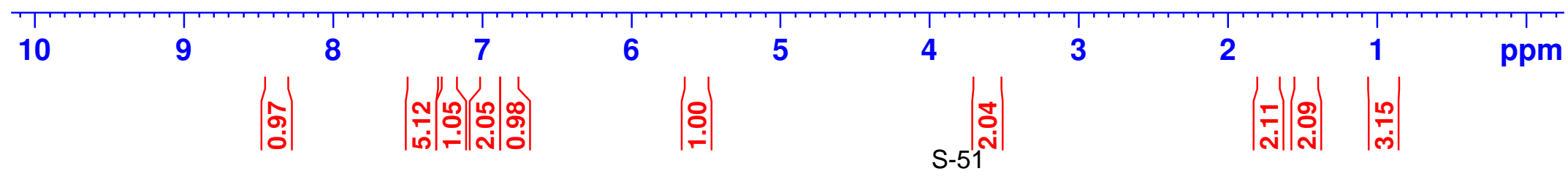



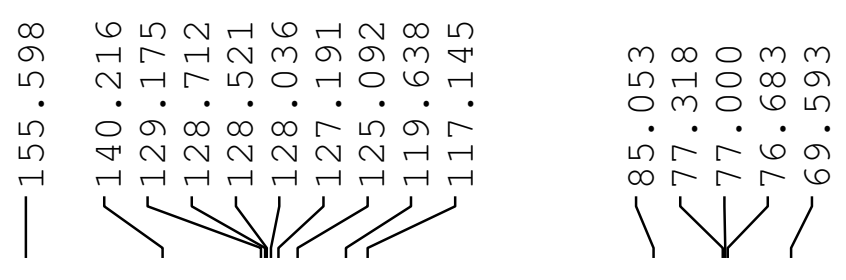

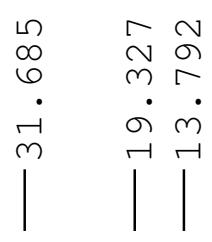

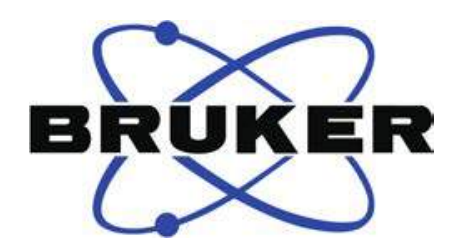

Current Data Parameters

NAME

EXPNO

lzw2102F-C<smiles>CCCCOC(c1ccccc1)c1ccccc1O</smiles>

$2 a$

F2 - Acquisition Parameters

Date__ 20150401

Time $\quad 13.20$

spect
INSTRUM $5 \mathrm{~mm} \mathrm{PABBO}$ BB

PUTPROG

zgpg30
65536

SOLVENT

SOLV
NS
DS
SWH

SWH

FIDRES

AQ

RG

$\mathrm{DW}$
$\mathrm{DE}$
$\mathrm{TE}$

D1
D11

D11

$=====$

$\mathrm{SFO1}$
$\mathrm{NUC1}$

$\mathrm{NUC1}$
$\mathrm{P} 1$

P1
PLW1

$\mathrm{CDCl} 3$

42

$24038.461 \mathrm{~Hz}$ $24038.461 \mathrm{~Hz}$
$0.366798 \mathrm{~Hz}$ $1.3631488 \mathrm{sec}$ 196.92 20.800 usec 6.50 usec
297.3 usec $297.3 \mathrm{~K}$

$2.00000000 \mathrm{sec}$ $0.03000000 \mathrm{sec}$ CHANNEL $\mathrm{fl}========$ $100.6228298 \mathrm{MHz}$ $13 \mathrm{C}$
9.70 usec (5) $\mathrm{SFO} 2$

NUC2

CPDPRG [2

PCPD2
PLW2

$P L W 12$
$P L W 13$

HANNEL f2 $========$
$400.1316005 \mathrm{MHz}$ 400.1316005
$1 \mathrm{H}$ waltz16
90.00 $11.99499989 \mathrm{~W}$ $0.34213999 \mathrm{~W}$ $0.27713001 \mathrm{~W}$

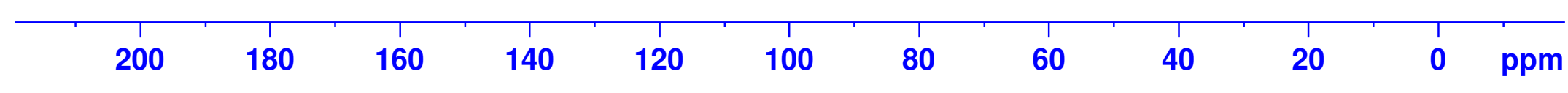

F2 - Processing parameters SI 32768 SF $\quad 100.6127753 \mathrm{MHz}$ 180 160

100

80




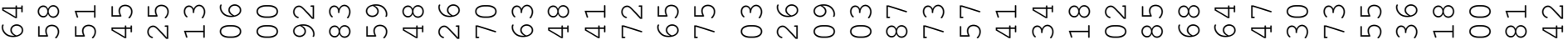

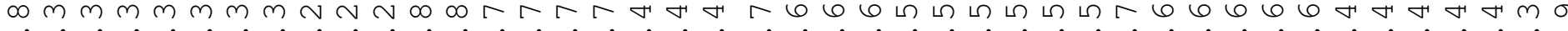
r r r r h r r

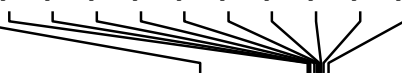

r

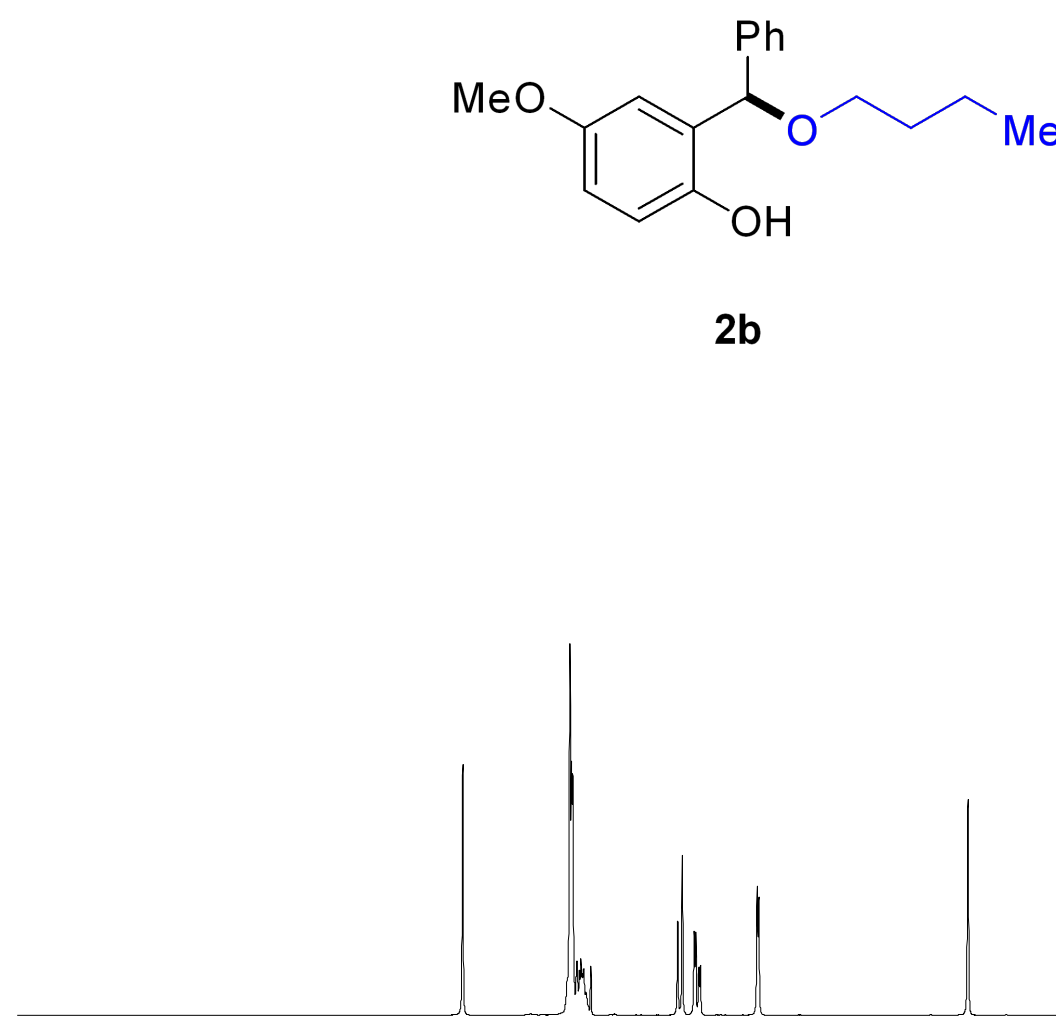

$2 b$
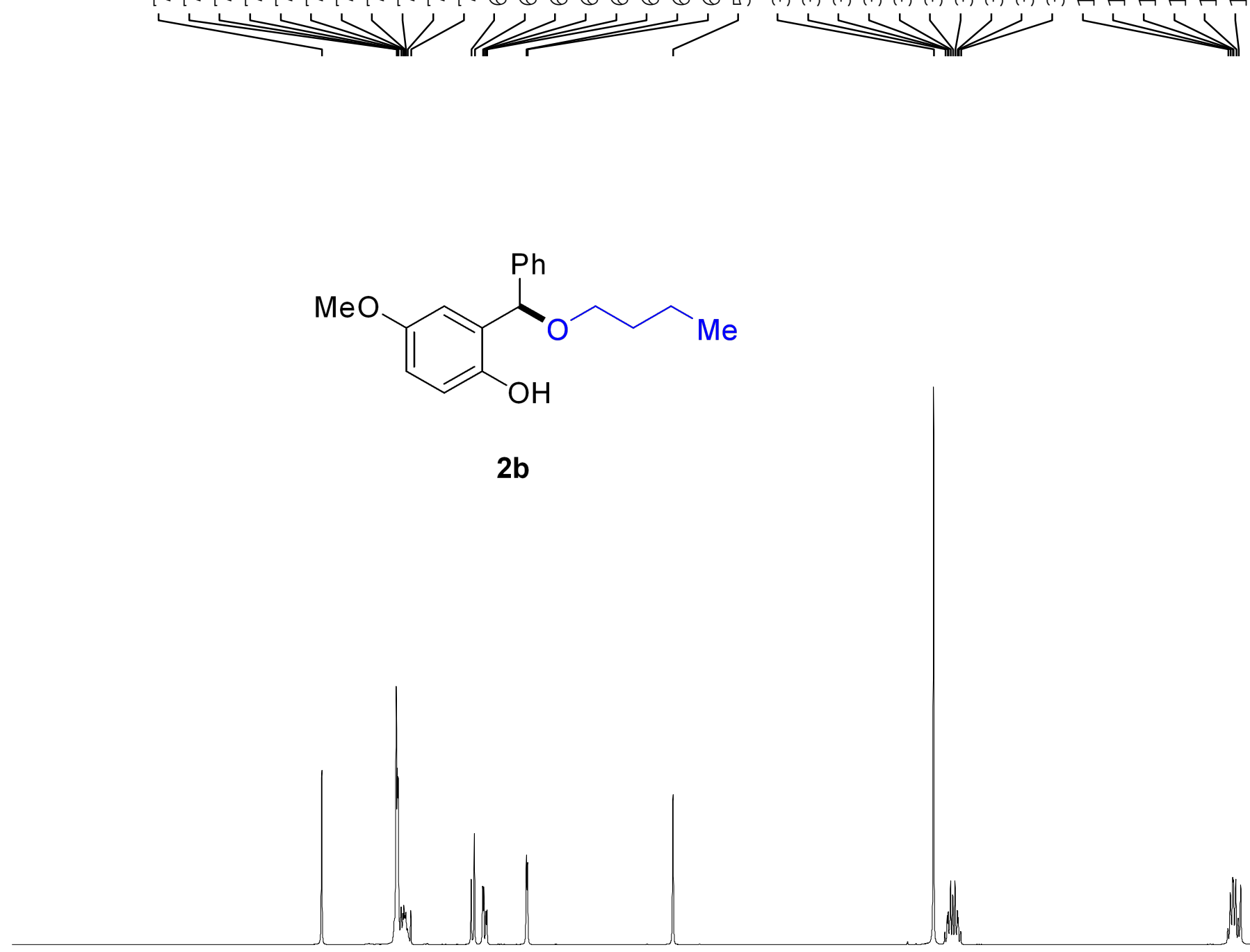

(1)

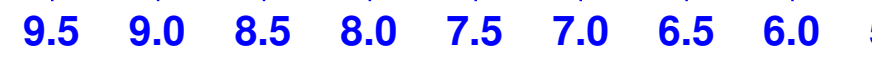

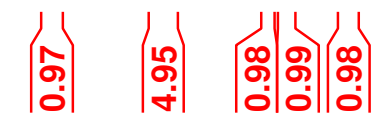

6.05 .5

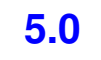

|임|

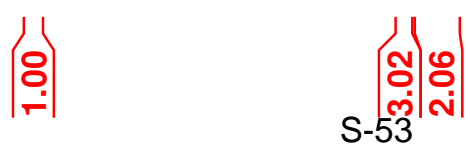

$3.0 \quad 2.5$

จ.

\section{Bporen $(1)$}

$\begin{array}{lr}\text { Current } & \text { Data } \\ \text { NAME } & \text { lzw2162D-H } \\ \text { EXPNO } & 1 \\ \text { PROCNO } & 1\end{array}$

F2 - Acquisition Parameters

Date_ $\quad 20150506$

$\begin{array}{ll}\text { Time } & 17.01\end{array}$

Spect

ROBHD

Zg30

$\begin{array}{ll}\text { TD } & 65536 \\ \text { SOLVENT } & \text { CDCl3 }\end{array}$

NS

DS

FIDRES

$\mathrm{AQ}$

RG

$\mathrm{DW}$
$\mathrm{DE}$
$\mathrm{TE}$

D 10

$===$

$\mathrm{SFO1}$

$\mathrm{NUC}$
$\mathrm{P} 1$

PLW1

$8012.820 \mathrm{~Hz}$

$0.122266 \mathrm{~Hz}$

54.81

62.400 usec

6.50 usec

$1.00000000 \mathrm{sec}$

$$
\begin{array}{r}
0 \\
1
\end{array}
$$

CHANNEL $\mathrm{fl}========$ $400.1324710 \mathrm{MHz}$ 14.50

50 use

$11.99499989 \mathrm{~W}$

F2 - Processing parameters

SI 65536

SF $\quad 400.1300096 \mathrm{MHz}$

EM

$0.30 \mathrm{~Hz}$

1.00 

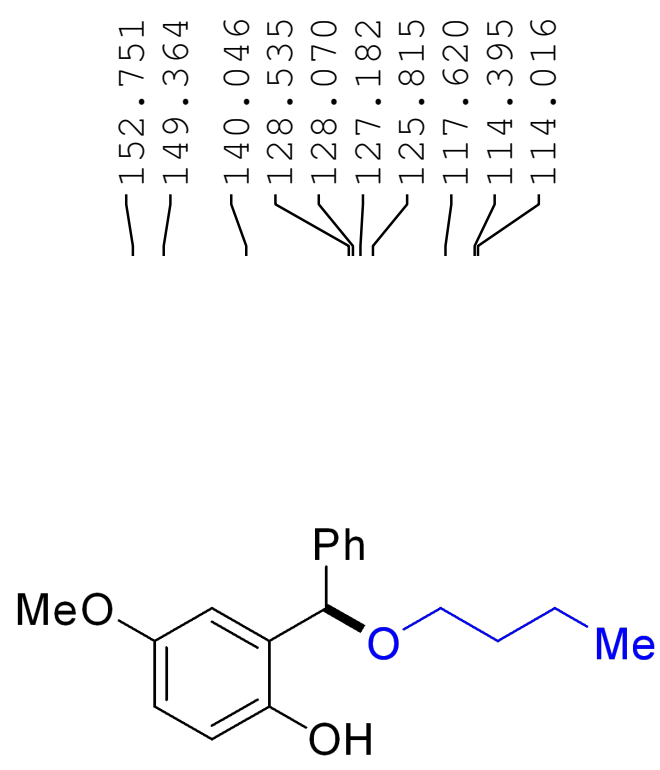

$2 b$

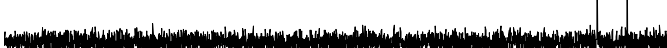

-

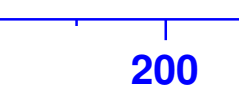

180

160

140

80

60

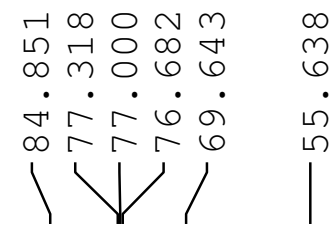

옹.

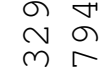

$\dot{\sigma} \dot{m}$

$i^{m}$

Current Data Parameters

NAME

EXPNO
PROCNO

Izw2162D-C

F2 - Acquisition Parameter

Date__ 20150506

$\begin{array}{lr}\text { Time } & 2017.04 \\ \text { INSTRUM } & \text { spect }\end{array}$

PROBHD

PULPROG

$5 \mathrm{~mm}$ PABBO BB/

SOLVENT

NS

DS

FID

FIDR
AQ

AQ

DW

$\mathrm{DE}$

$\mathrm{TE}$
$\mathrm{D} 1$
$\mathrm{D} 11$

D11

TD 0

$======$

$\mathrm{SFO1}$

NUC

P 1
PLW1

$\mathrm{CDCl} 3$

48

$24038.461 \mathrm{~Hz}$

$1.3631488 \mathrm{sec}$

196.92

20.800 usec

6.50 usec

$297.3 \mathrm{~K}$

$2.00000000 \mathrm{sec}$ $0.03000000 \mathrm{sec}$

CHANNET 61 = $==0=$ $100.6228298 \mathrm{MHz}$ $13 \mathrm{C}$ 9.70 usec

$=======$ CHANNEL $\mathrm{f} 2========$ $\mathrm{SFO} 2$

NUC2

CPDPRG [2

PCPD2

PLW2

PLW12

PLW13

$400.1316005 \mathrm{MHz}$

$1 \mathrm{H}$

90.00 usec

$11.99499989 \mathrm{~W}$

$0.34213999 \mathrm{~W}$
$0.27713001 \mathrm{~W}$

F2 - Processing parameters SI $\quad 32768$

SF $\quad 100.6127737 \mathrm{MHz}$

SSB

$\mathrm{EM}$
$\mathrm{EM}$ 


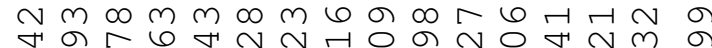
Hmmmmmm m n

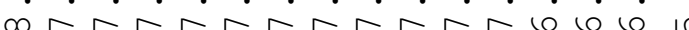
$\longrightarrow$

स $\infty$ -

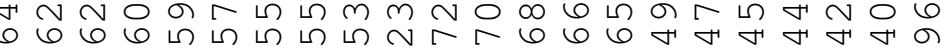

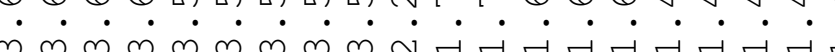
में

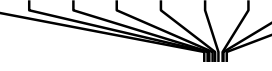<smiles>CCCCOC(c1ccccc1)c1cc(C)ccc1O</smiles>

$2 c$

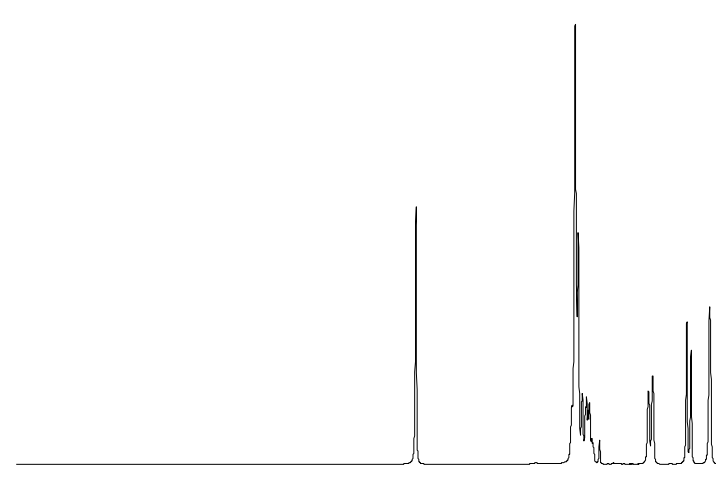
7

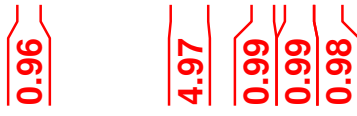

6

$\left|\begin{array}{l}\mid \\ \delta \\ \hdashline\end{array}\right|$ ᄂ
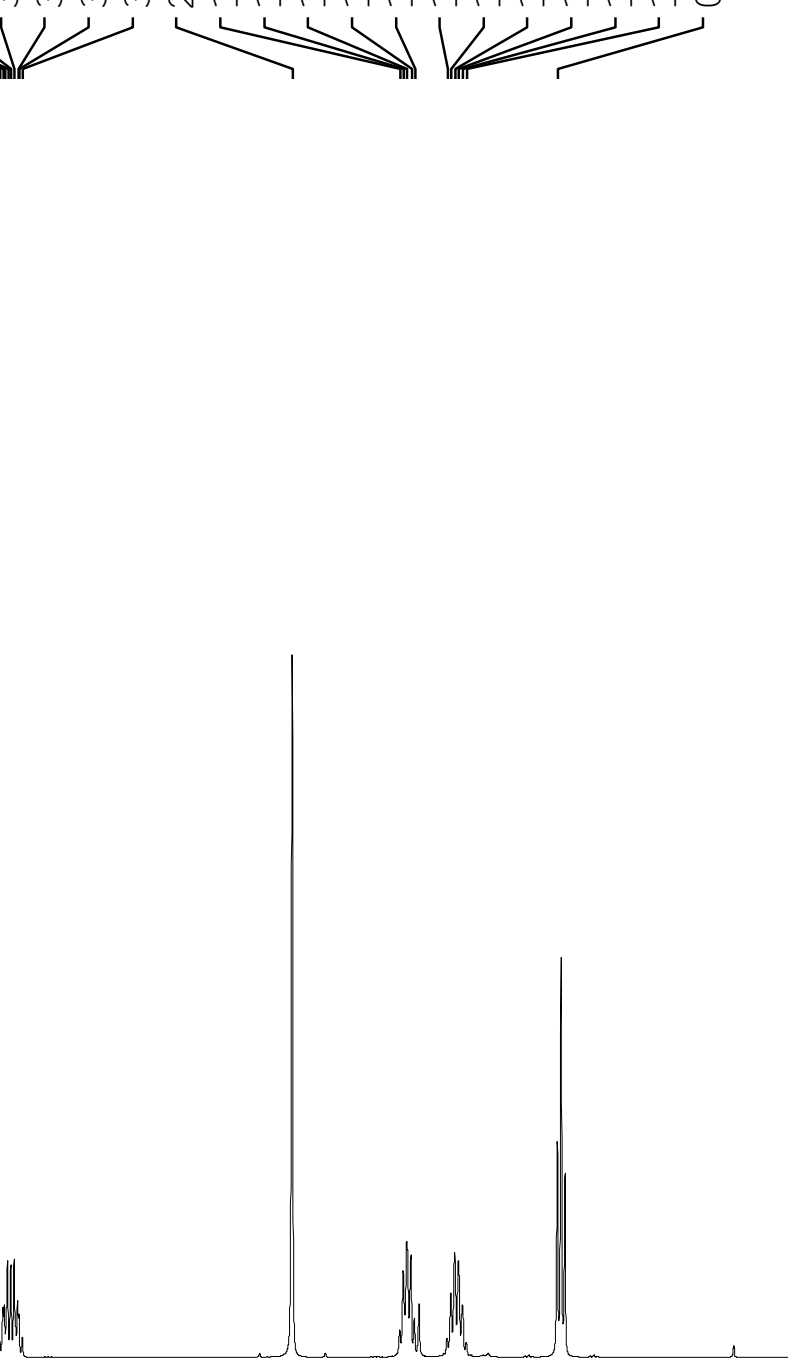

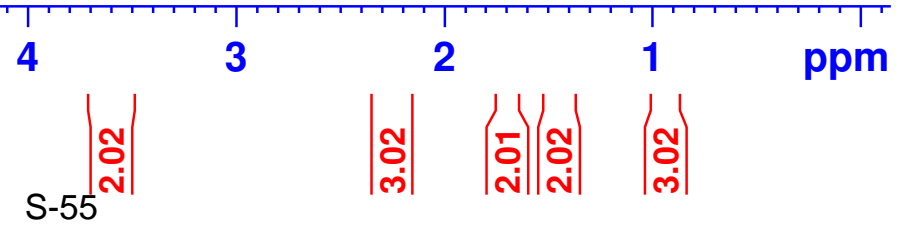

\section{experer $(1)$}

Current Data Parameters NAME

PROCNO

lzw2180B-H

F2 - Acquisition Parameters

Date__ 20150514

Time $\quad 16.00$

$5 \mathrm{spect}$

5 mm
PULPROG

$\begin{array}{lr}\text { TD } & \text { Zg30 } \\ & 65536\end{array}$

SOLVENT

NS

DS

$\begin{array}{ll}\text { SWH } & 8012.820 \mathrm{~Hz} \\ \text { FIDRES } & 0.122266 \mathrm{~Hz}\end{array}$

$\mathrm{DW}$

$\mathrm{DE}$

D1

TD 0

$===$

$\mathrm{SFO1}$

$\mathrm{NUC}$

PLW1

$65 \mathrm{sec}$ 31.55

62.400 usec
6.50 usec

$1.00000000 \mathrm{sec}$ 1

CHANNEL $\mathrm{fl}========$ $400.1324710 \mathrm{MHz}$ 14.50 usec

$2-P r o d(x)$

- Processing parameter

SI 65536

SF $400.1300088 \mathrm{MHz}$

SSB

EM

$0.30 \mathrm{~Hz}$

1.00 
न ॠ

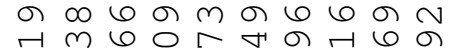

$\dot{m} \dot{\circ} \dot{\sigma} \dot{\sigma} \dot{\infty} \dot{\sim} \dot{\sim} \dot{\sigma}$

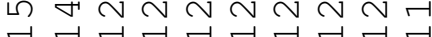

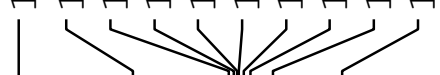

(3)

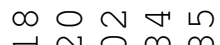

다 $\circ 6$ เ

$\dot{0} \dot{\sim} \dot{\circ} \dot{\sigma}$

111

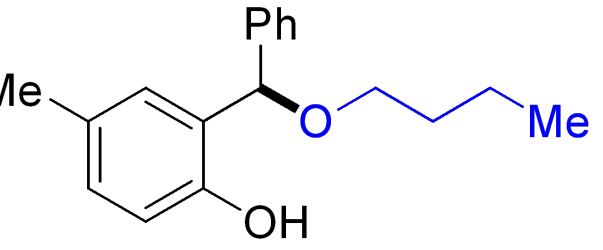

2c

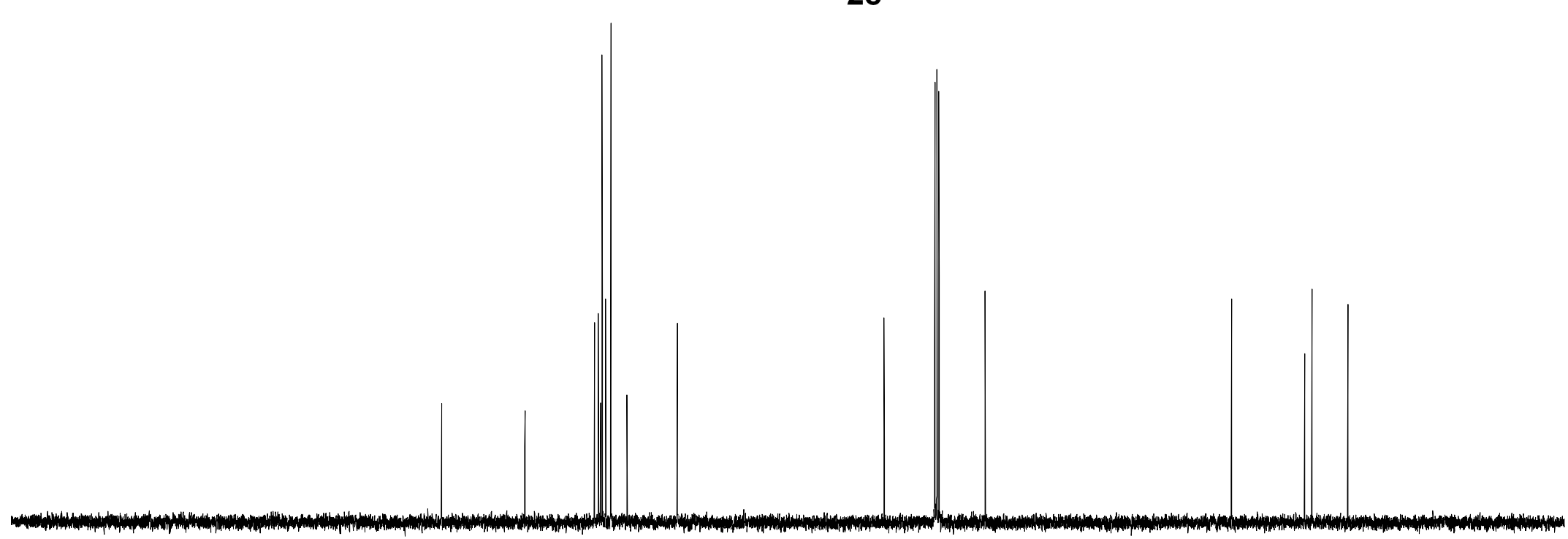

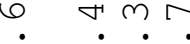

가 $\circ$ न $m$

111
ำ

\section{Bg 11}

Current Data Parameters NAME

EXPNO

lzw2180B-C

PROCNO

F2 - Acquisition Parameter

Date_

20150514

Time

PROBHD

PULPROG

TD

SOLVENT

NS

DS

SWH

$\mathrm{AQ}$

RG
DW
DE

$\mathrm{TE}$

D11

TD 0

$======$

$\mathrm{SFO1}$

P 1
PLW1

$100.6228298 \mathrm{MH}$ $13 \mathrm{C}$

$========$ CHANNEL $\mathrm{f} 2========$ SEO2

NUC2

CPDPRG [2

PCPD2

LLW2

LW12

PLW13

$400.1316005 \mathrm{MHz}$

$1 \mathrm{H}$

waltz16

$11.99499989 \mathrm{~W}$ $0.34213999 \mathrm{~W}$

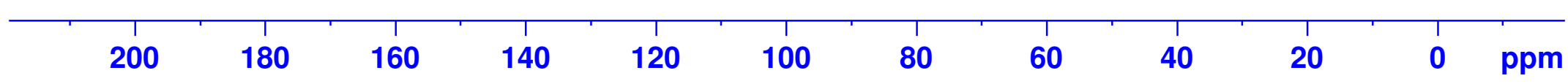

2 - Processing parameters

SI $\quad 32768$

$100.6127773 \mathrm{MHz}$

SS
LB
GB

$1.00 \mathrm{~Hz}$

1.40 
レの

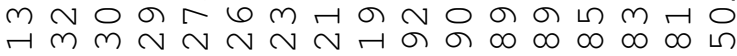

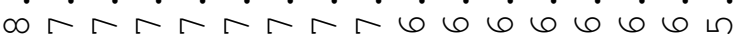

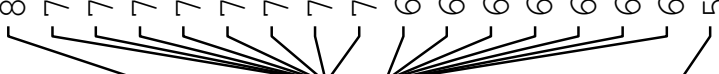

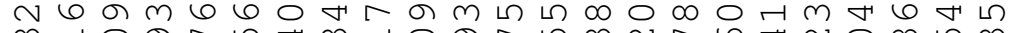
m . . . . . . . $m m m m m m m m-r+H-r+r$

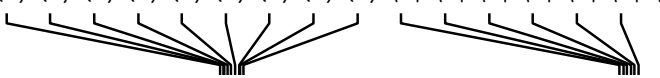

(n)

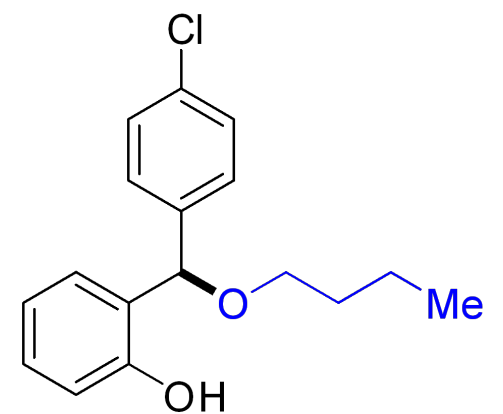

2d

\section{BR WXER

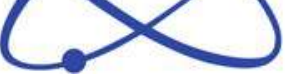

Current Data Parameters NAME

PROCNO

lzw2156B-H

F2 - Acquisition Parameters

Date_ 20150502

Time $\quad 15.29$

$5 \mathrm{~mm}$ spect

PULPROG $\quad$ zg30

$\begin{array}{lr}\mathrm{Zg} 30 \\ \mathrm{TD} & 65536\end{array}$

SOLVENT $\quad$ CDCl3

DS

SWH

FIDRES

$\mathrm{AQ}$

RG

DW

$\mathrm{DE}$

D1
TD 0

$====$

$\mathrm{SFO1}$

$\mathrm{NUC1}$
$\mathrm{P} 1$

P 1
PLW1

0
$8012.820 \mathrm{~Hz}$

$4.0894465 \mathrm{sec}$

39.46

62.400 usec

6.50 usec

$1.00000000 \mathrm{sec}$

$$
1
$$

CHANNEL $\mathrm{f1}========$

$400.1324710 \mathrm{MHz}$ $1 \mathrm{H}$

$11.99499989 \mathrm{~W}$

F2 - Processing parameters

SI 65536

SF $\quad 400.1300092 \mathrm{MHz}$

WDW

EM

$0.30 \mathrm{~Hz}$

1.00 

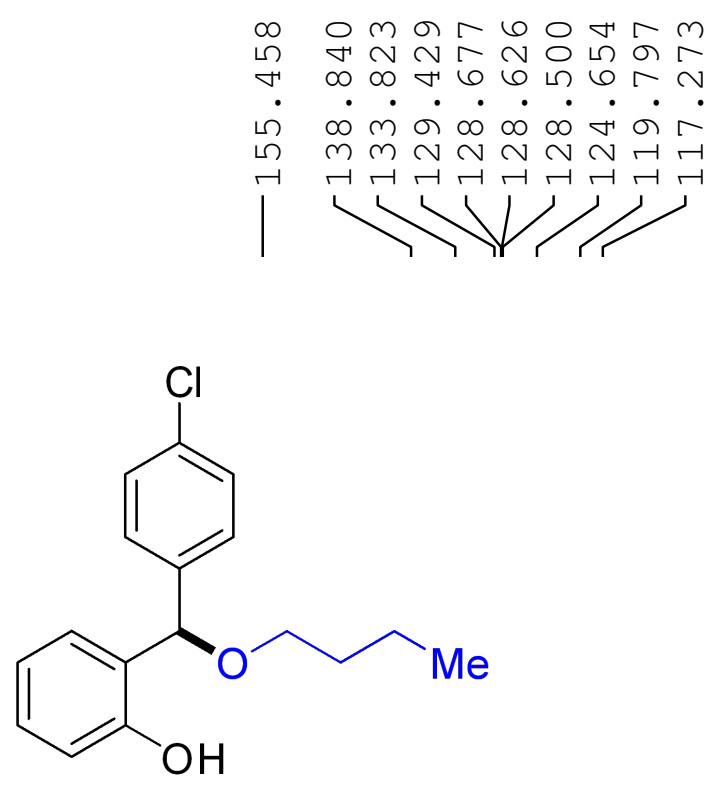

2d $\begin{array}{llll}2 & 0 & 0 \\ 2 m & 0 & -4\end{array}$

$\rightarrow m 0, \pi$

$\dot{\sim} \sim \dot{\sim} \dot{0}$

$|\bigvee|$

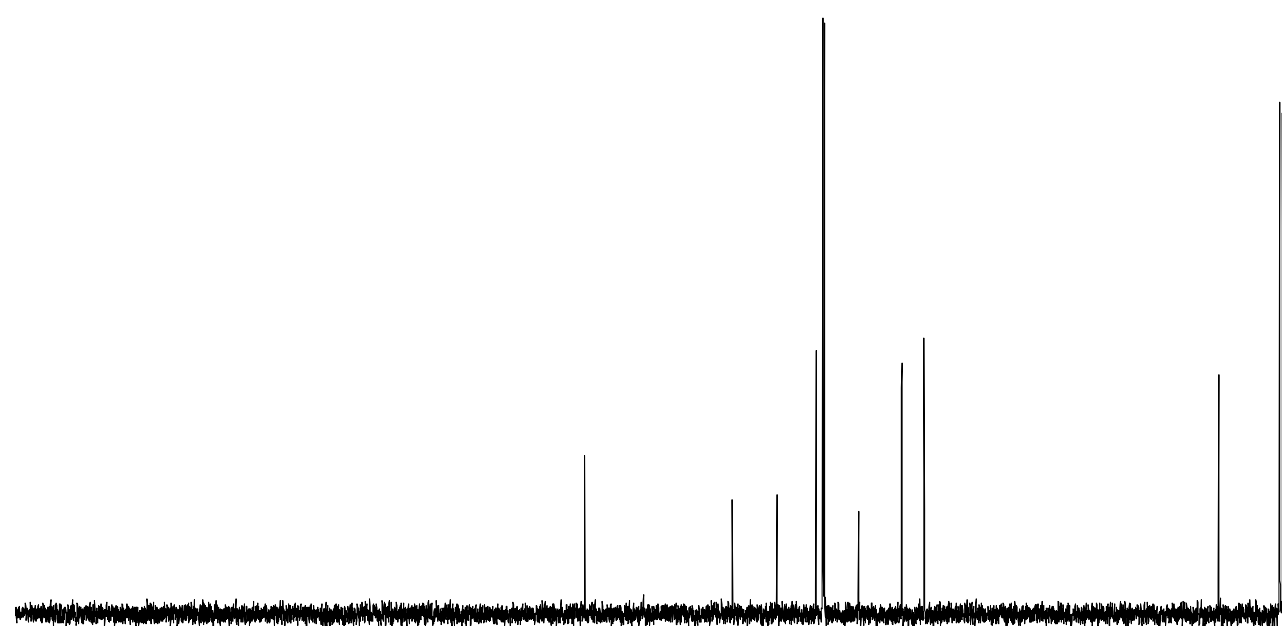

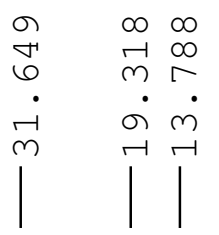

Current Data Parameters NAME

EXPNO

lzw2156B-C

PROCNO

F2 - Acquisition Parameters

Date__ 20150502

$\begin{array}{ll}\text { Time } & 15.32\end{array}$

SPect

PROBHD

Zgpg30

$\begin{array}{lr}\text { TD } & 65536 \\ \text { SOLVENT } & \text { CDCl3 } \\ \text { NS } & 20\end{array}$

DS

SWH

FIDRES

AQ

RG

DW
DE
TE

D1

D11

$======$

$\mathrm{SFO} 1$

NUC

P 1

PLW1

$24038.461 \mathrm{~Hz}$

$\begin{array}{rl}0.366798 & \mathrm{~Hz} \\ 1.3631488 \mathrm{sec}\end{array}$

196.92

20.800 usec

6.50 usec

$296.8 \mathrm{~K}$

$2.00000000 \mathrm{sec}$
$0.03000000 \mathrm{sec}$

CHANNET 11 $==2==$ $100.6228298 \mathrm{MHz}$ $13 \mathrm{C}$

9.70 usec
$46.98899841 \mathrm{~W}$

$=======$ CHANNEL $\mathrm{f} 2========$ $\mathrm{SFO} 2 \quad 400.1316005 \mathrm{MHz}$

NUC2

CPDPRG [2

PCPD2

PLW2

PLW12

PLW13

waltz16

90.00 usec

$11.99499989 \mathrm{~W}$

$0.34213999 \mathrm{~W}$
$0.27713001 \mathrm{~W}$

F2 - Processing parameters SI $\quad 32768$ 


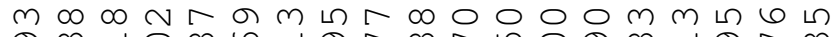
๙

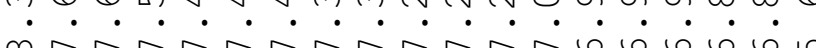

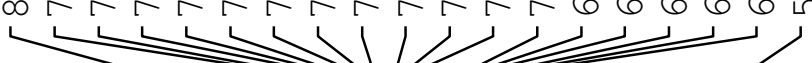

m

6666

$\dot{m} \dot{m} \dot{m} \dot{m}$

No सr

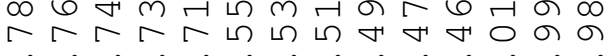
- 4 $\ldots$
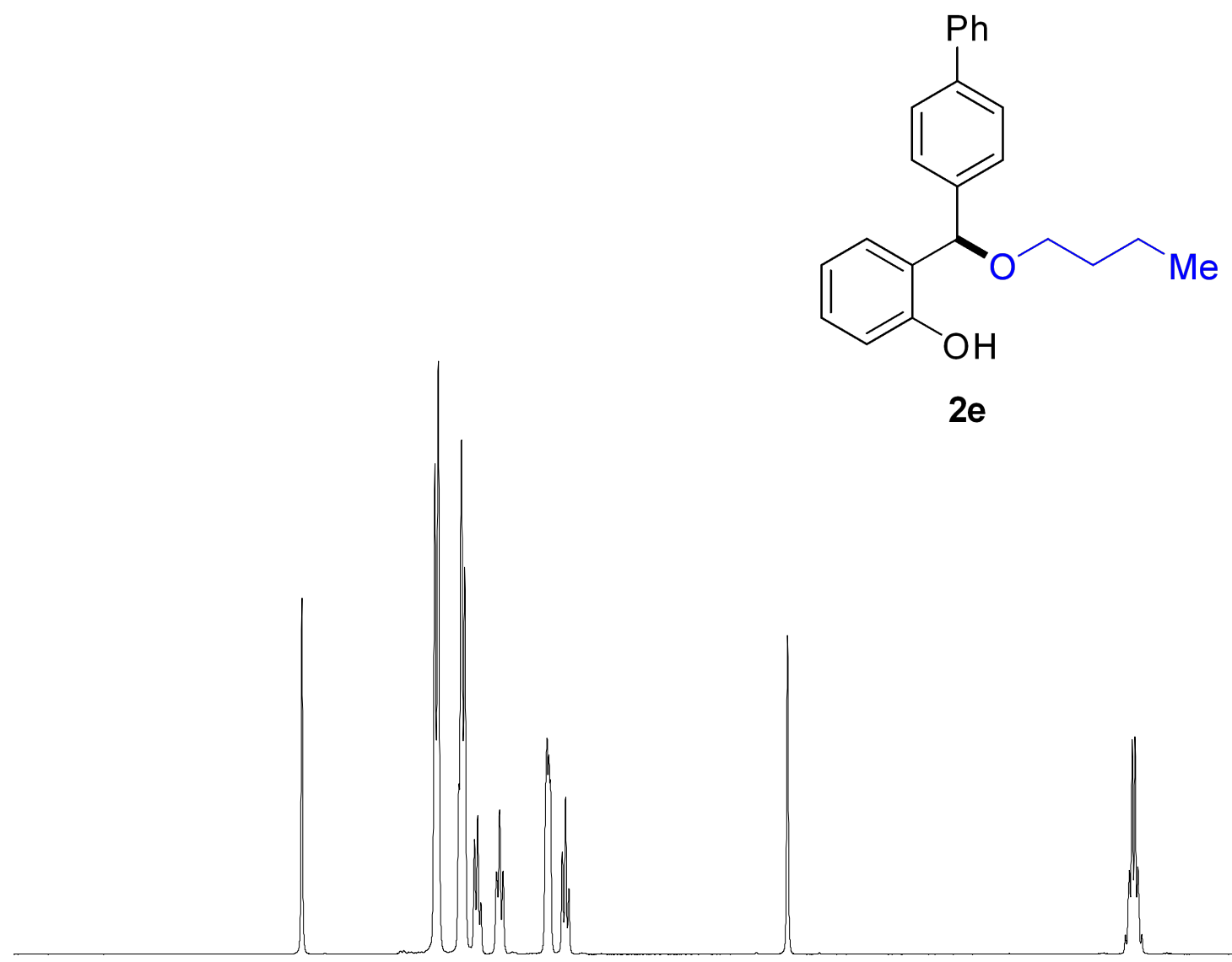

|

\begin{abstract}
6
\end{abstract}
|이미

$2 e$
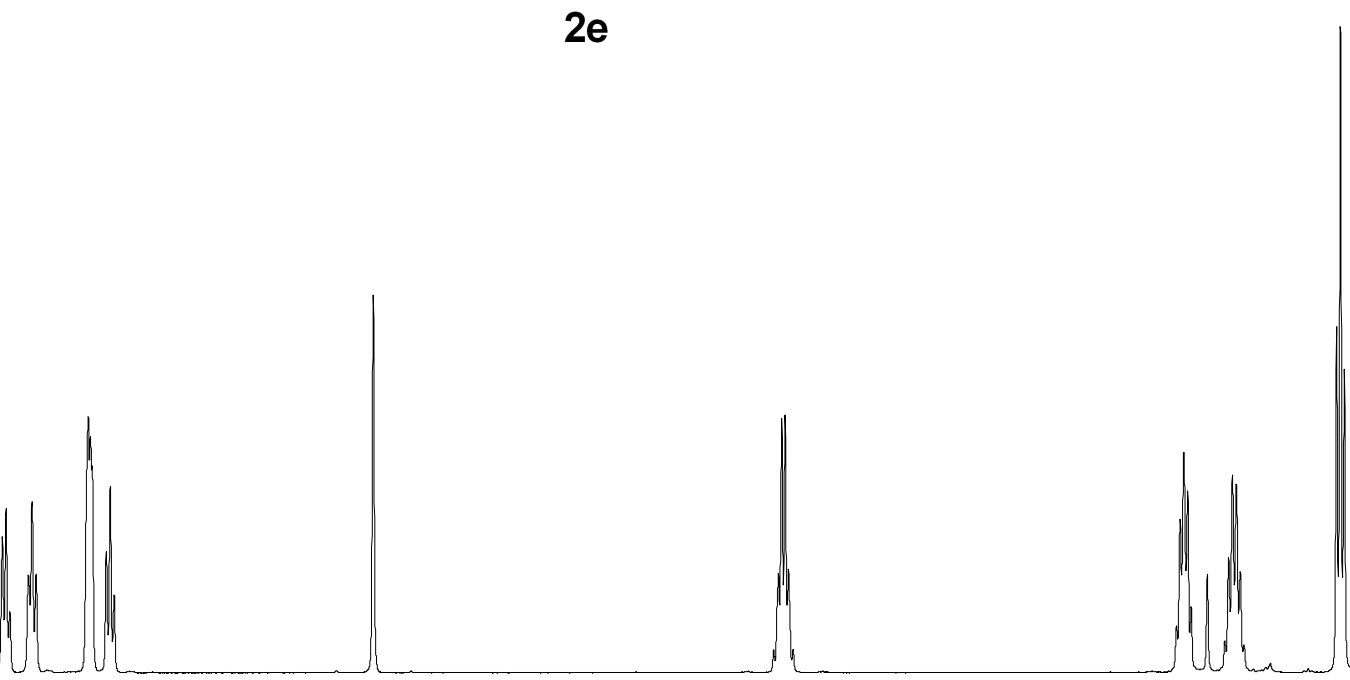

\section{BD WXER

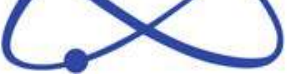

Current Data Parameters NAME

EROCNO

lzw2170C-H

F2 - Acquisition Parameters

Date_ 20150509

Time

20.06

INSTRUM

PROBHD

$5 \mathrm{~mm} \mathrm{PABBO} \mathrm{BB} /$

PULPROG

zg 30
65536

SOLVENT

NS

DS

SWH

IDR

$\mathrm{AQ}$

RG

$\mathrm{DW}$
$\mathrm{DE}$

$\mathrm{TE}$
$\mathrm{D} 1$

TD 0

$===$

$\mathrm{SFO1}$

$\mathrm{NUC1}$
$\mathrm{P} 1$

PLW1

zg30
65536
CDC13

0
0
$8012.820 \mathrm{~Hz}$

$4.0894465 \mathrm{~Hz}$

31.55

62.400 usec

6.50 usec

$1.00000000 \mathrm{~K}$

$$
1
$$

CHANNEL $\mathrm{f1}========$

$400.1324710 \mathrm{MHz}$ $1 \mathrm{H}$

$11.99499989 \mathrm{~W}$

F2 - Processing parameters

SI 65536

SF $\quad 400.1300000 \mathrm{MHz}$

EM

$0.30 \mathrm{~Hz}$

1.00 
๙ૅ

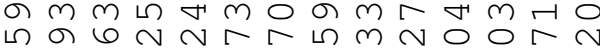

ம் $\dot{0} \dot{\sigma} \dot{\sigma} \dot{0} \dot{\sim} \dot{\sim} \dot{\sim} \dot{\sigma} \dot{\sim}$

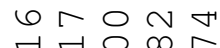

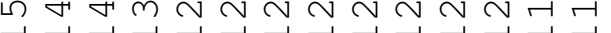

$m \circ 66$

$\forall \therefore \therefore \dot{\sigma}$

$|V|$

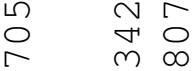

$\dot{r} \quad \dot{m} \dot{m}$

$\longrightarrow$

$1 \mid$
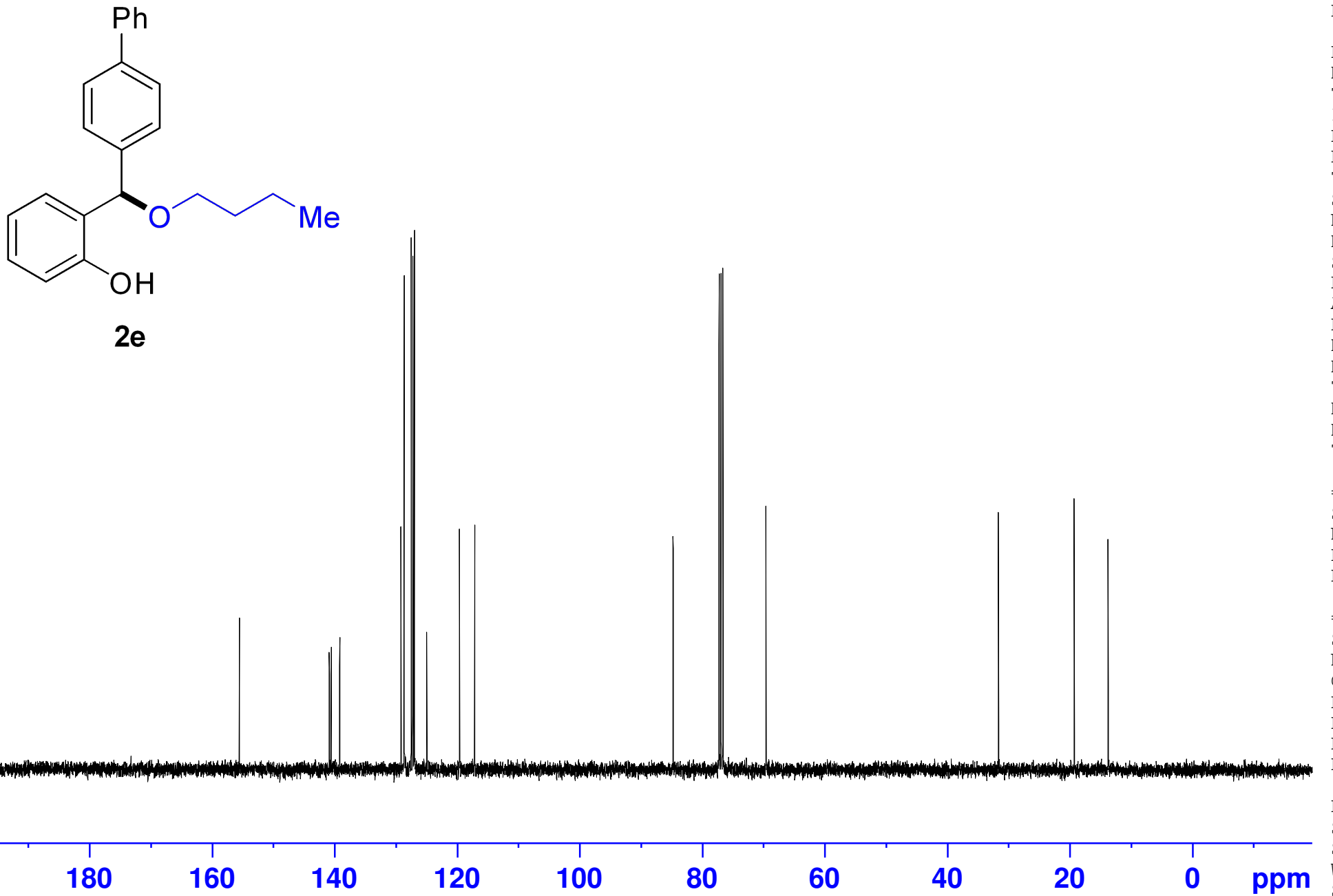

200

80 


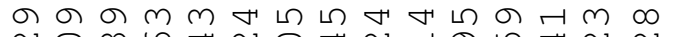

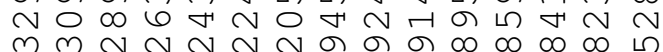
$\dot{0} \cdot \cdot \cdot \cdot \cdot 0$

$>_{m}^{\infty}$

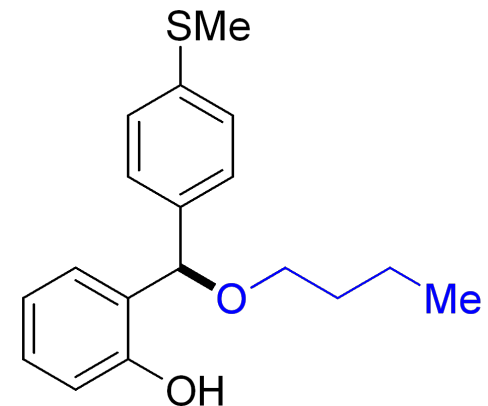

$2 f$

군

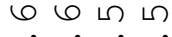

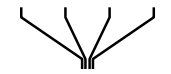

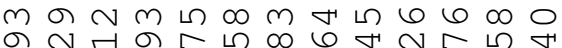
次 N$\longrightarrow$

\section{aporer $(1)$}

Current Data Parameters NAME

EROCNO

lzw2150C-H

F2 - Acquisition Parameters

Date__ 20150429

Time 21.48

$5 \mathrm{mmect}$

PULPROG

$\mathrm{Zg} 30$
65536

TD $\quad 65536$

NS

DS

FIDRES

$\mathrm{AQ}$

RG

DW

$\mathrm{DE}$

TE

TD 0

$===$

$\mathrm{SFO}$

$\mathrm{NUC1}$

$\mathrm{CDC} 13$
7

\section{$8012.820 \mathrm{~Hz}$}

$0.122266 \mathrm{~Hz}$

$4.0894465 \mathrm{sec}$ 31.55

62.400 usec 6.50 usec

$1.00000000 \mathrm{sec}$ 1

CHANNEL $\mathrm{fl}========$ $400.1324710 \mathrm{MHz}$ 14.50

PLW1 $11.99499989 \mathrm{~W}$

F2 - Processing parameters

SI $\quad 65536$

SF $\quad 400.1300000 \mathrm{MHz}$

WDW EM

$0.30 \mathrm{~Hz}$

1.00

\section{8}

|นิำ.

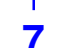

|

6

$\left|\begin{array}{l}8 \\ 8 \\ -1\end{array}\right|$
5

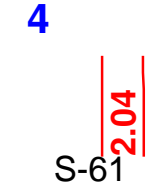

3

$\left|\begin{array}{l}1 \\ 0 \\ 0\end{array}\right|$

2

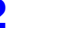
|이 ppm 

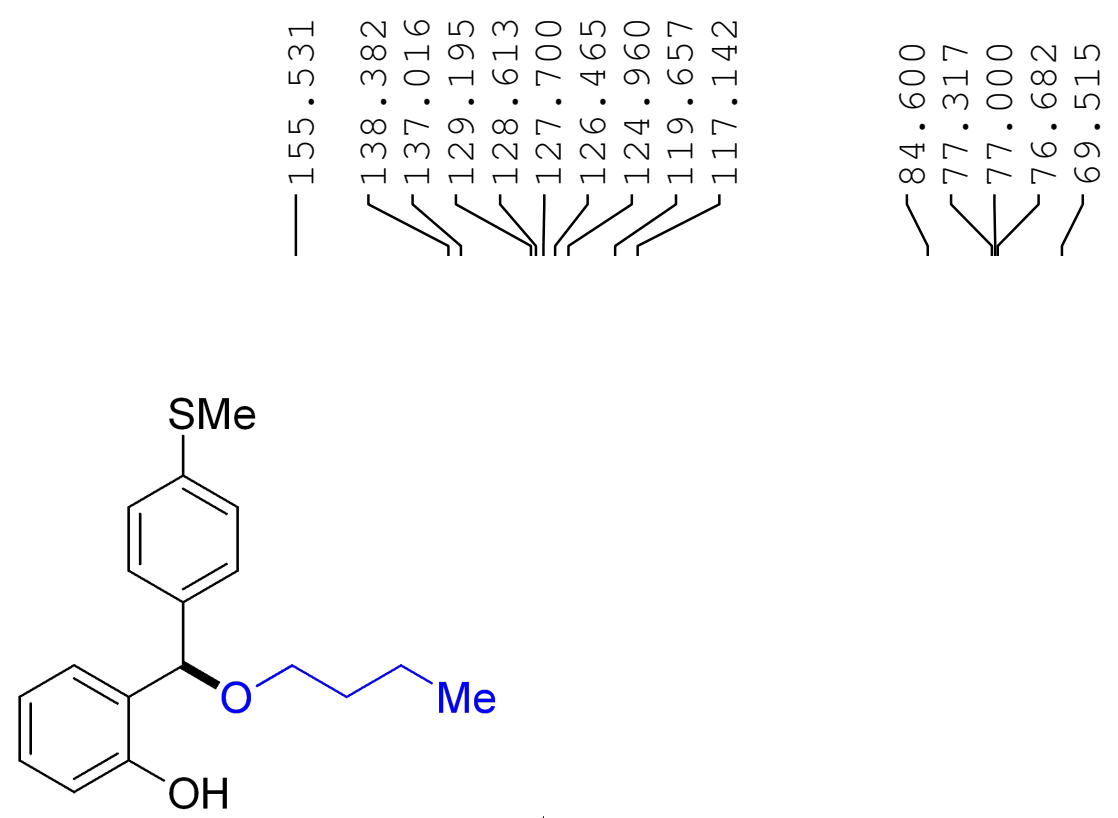

$2 f$
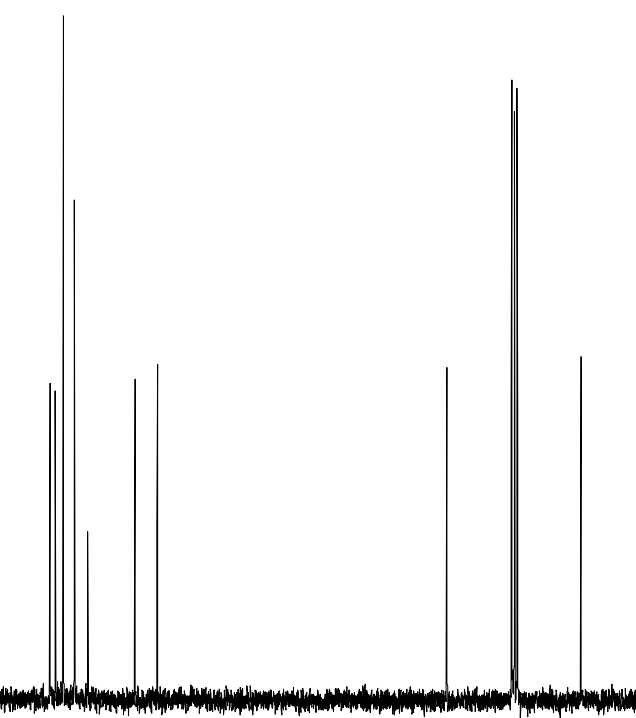

160

140

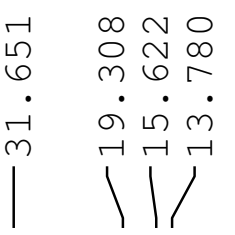

NAME

EXPNO

ROCNO

F2 - Acquisition Parameters

Date

20150429

Time

INSTRUM

PULPROG

SOLVENT

NS

DS

SWH

IDRES

$\mathrm{AQ}$

RG

DW

DE

D1
D11
TD0

$======$

$\mathrm{SFO1}$
NUC1

P 1
PLW1

$5 \mathrm{~mm}$ PABBO BB/

zgpg 30
65536

26

$24038.461 \mathrm{~Hz}$

$1.366798 \mathrm{~Hz}$

196.92

20.800 usec 6.50 usec

$297.2 \mathrm{~K}$

$2.00000000 \mathrm{sec}$ $0.03000000 \mathrm{sec}$

CHANNET $61=$ $100.6228298 \mathrm{MHz}$ $13 \mathrm{C}$

9.70 usec
$46.98899841 \mathrm{~W}$ $=======$ CHANNEL $\mathrm{f} 2$ $=======$

NUC2

CPDPRG [2

PCPD2

PLW2

PLW12

PLW13

$400.1316005 \mathrm{MHz}$ $1 \mathrm{H}$ waltzl6 0.00 usec $11.99499989 \mathrm{~W}$ $0.34213999 \mathrm{~W}$ $0.27713001 \mathrm{~W}$ 
৮다 ம न $\infty \infty$ เ

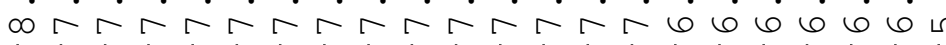
- $\infty$ の

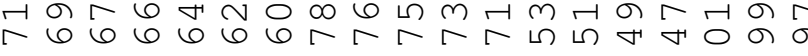

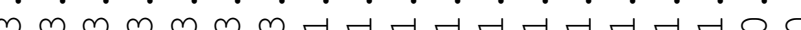
$\gg<1$ M.
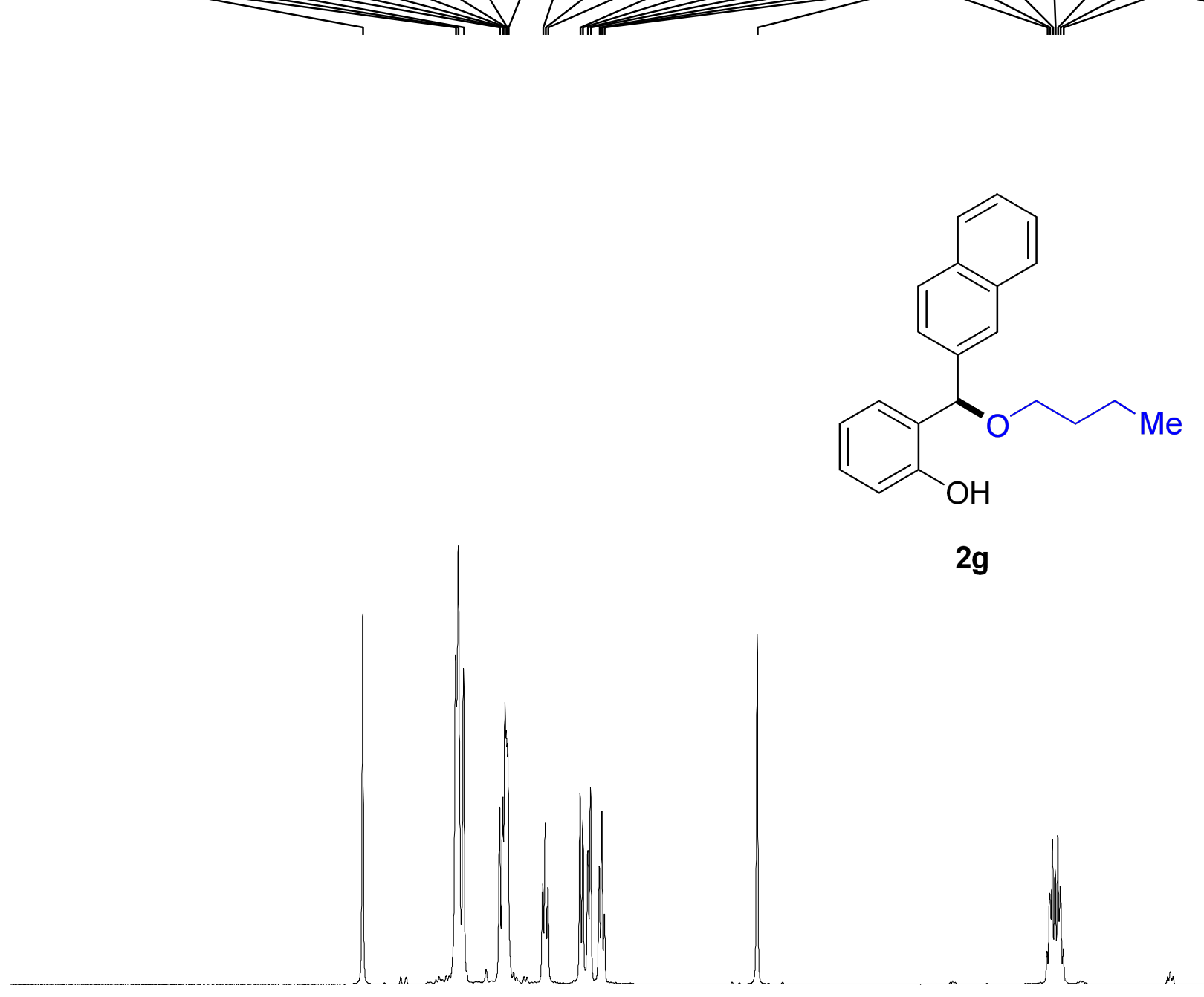

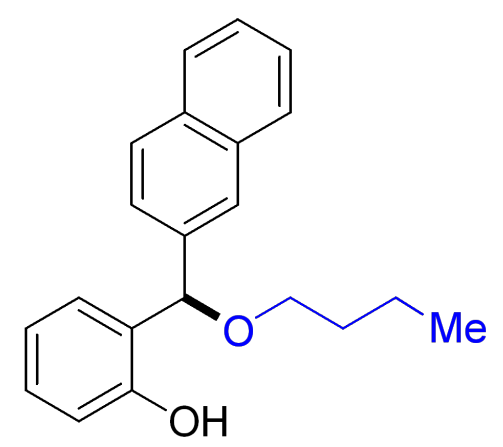

$2 \mathrm{~g}$

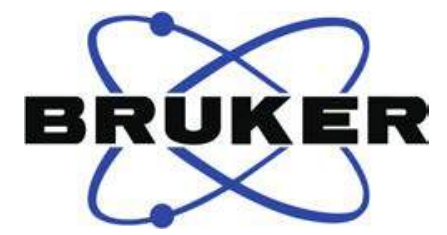

Current Data Parameters NAME PROCNO

F2 - Acquisition Parameter Date_ 20150527 $\begin{array}{lr}\text { Time- } & 19.41\end{array}$ spect Zg30
PULPROG $\begin{array}{ll}\text { TD } & 65536 \\ \text { SOLVENT } & \text { CDCl3 }\end{array}$ NS $4.0894465 \mathrm{~Hz}$ $\mathrm{DE}$ 62.400 usec
6.50 usec $296.3 \mathrm{~K}$ TDO $======$ CHANNEI $\mathrm{fl}=======0$ SFO1 $400.1324710 \mathrm{MHz}$ 13.50 11.9949998 usec PLW1 11.99499989 W

F2 - Processing parameters SI 65536 SF $400.1300000 \mathrm{MHz}$ WDW EM

SSB

$0.30 \mathrm{~Hz}$

1.00

| 


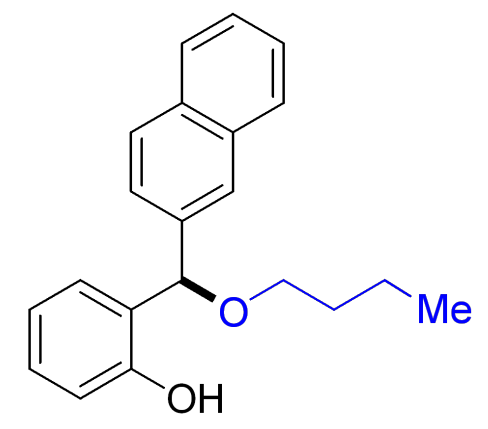

$2 \mathrm{~g}$

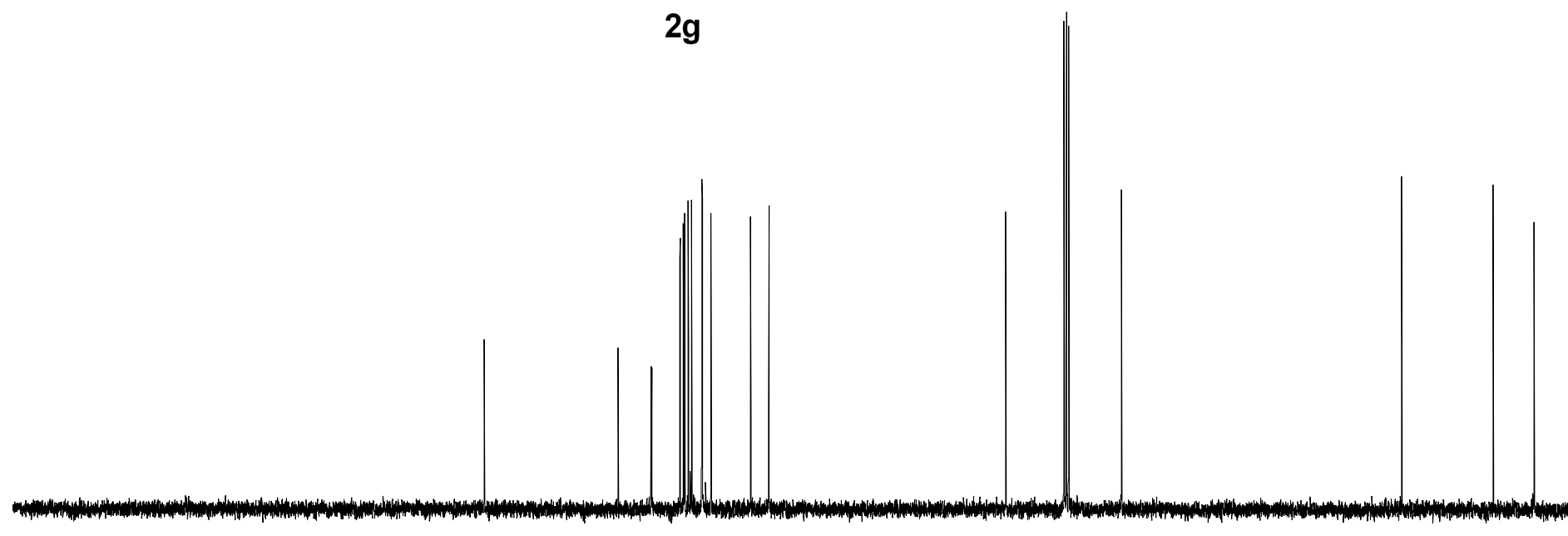

\section{BD $(1)$}

Current Data Parameters

F2 - Acquisition Parameters

Date_ 20150527

Time $\quad 19.44$

spect
INSTRUM $5 \mathrm{~mm}$ PABBO BB/

PULPROG

zgpg30

$\begin{array}{ll}\text { TD } & 65536 \\ \text { SOLVENT } & \text { CDC13 }\end{array}$

NS

DS

FID

FIDR
AQ
RG

DW

$\mathrm{DE}$

TE

D1 1

27
0

$24038.461 \mathrm{~Hz}$ $0.366798 \mathrm{~Hz}$

$1.3631488 \mathrm{sec}$ 196.92

20.800 usec 6.50 usec $2.00000000 \mathrm{~K}$ $2.00000000 \mathrm{sec}$ $0.03000000 \mathrm{sec}$ TD 0

\section{$=====$}

$\mathrm{SFO1}$

$\mathrm{NUC} 1$
$\mathrm{P} 1$

CHANNEL $\mathrm{f1}========$

$100.6228298 \mathrm{MHz}$

PLW1

$46.98899841 \mathrm{~W}$

$\mathrm{SFO} 2$

NUC2

CPDPRG [2

PCPD2

PLW2

PLW12

PLW13

NNEL 12 ======== $400.1316005 \mathrm{MHz}$ $1 \mathrm{H}$

90.00 usec $11.99499989 \mathrm{~W}$ $0.34213999 \mathrm{~W}$
$0.27713001 \mathrm{~W}$

\begin{tabular}{|c|c|c|c|c|c|c|c|c|c|c|}
\hline 200 & $\begin{array}{r}180 \\
180\end{array}$ & 160 & 140 & $\begin{array}{c}1 \\
120\end{array}$ & 100 & 80 & 60 & 40 & 20 & $\begin{array}{l}1 \\
0\end{array}$ \\
\hline
\end{tabular}

F2 - Processing parameters SI $\quad 32768$ $\begin{array}{ll}S F & 100.6127813 \mathrm{MHz}\end{array}$

酸


の 담

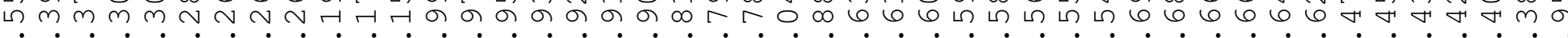
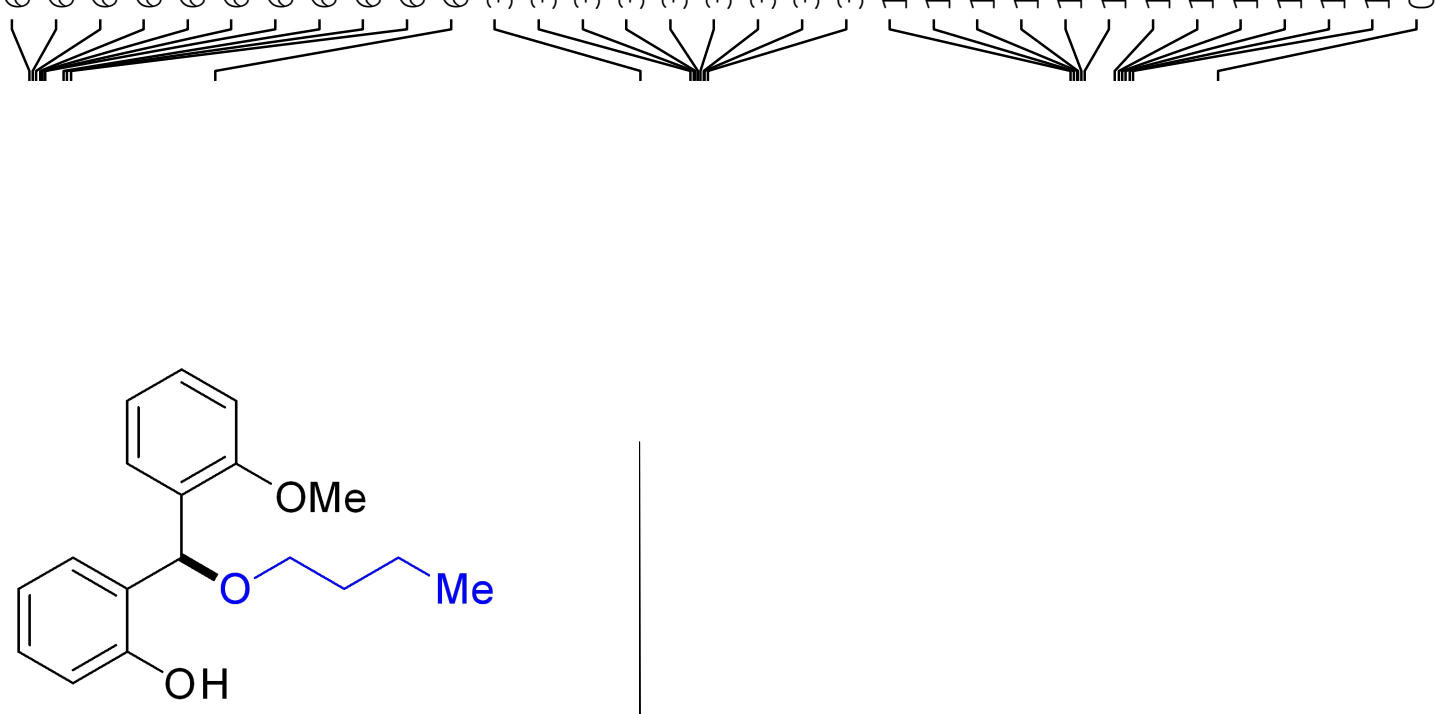

$2 \mathrm{~h}$

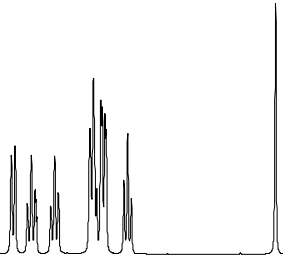

8

$\left|\begin{array}{c}\infty \\ 0 \\ 0\end{array}\right|$

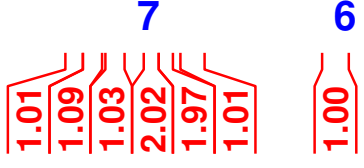

65

4

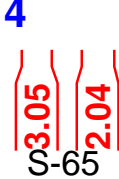

\section{aporen $(->)$}

Current Data Parameters NAME

lzw2176D-H

PROCNO

F2 - Acquisition Parameters

Date_ 20150512

Time $\quad 13.56$

spect

PULPROG
zg30

$\begin{array}{lr}\text { TD } & \text { Zg30 } \\ & 65536\end{array}$

$\begin{array}{ll}\text { TD } & 65536 \\ \text { SOLVENT } & \text { CDCl3 }\end{array}$

NS

DS

FIDRES

$\mathrm{AQ}$

RG

DW

$\mathrm{DE}$

D1
TD 0

$====$

$\mathrm{SFO1}$

$\mathrm{NUC}$
$\mathrm{P} 1$

6
0

$8012.820 \mathrm{~Hz}$

$0.122266 \mathrm{~Hz}$

$4.0894465 \mathrm{sec}$ 34.77

62.400 usec 6.50 use

$1.00000000 \mathrm{sec}$

$$
1
$$

CHANNEL $\mathrm{f} 1========$ $400.1324710 \mathrm{MHz}$ 14.50

50 use

PLW1 $11.99499989 \mathrm{~W}$

F2 - Processing parameters

SI $\quad 65536$

SF $\quad 400.1300089 \mathrm{MHz}$

SSB

EM

$0.30 \mathrm{~Hz}$

1.00 


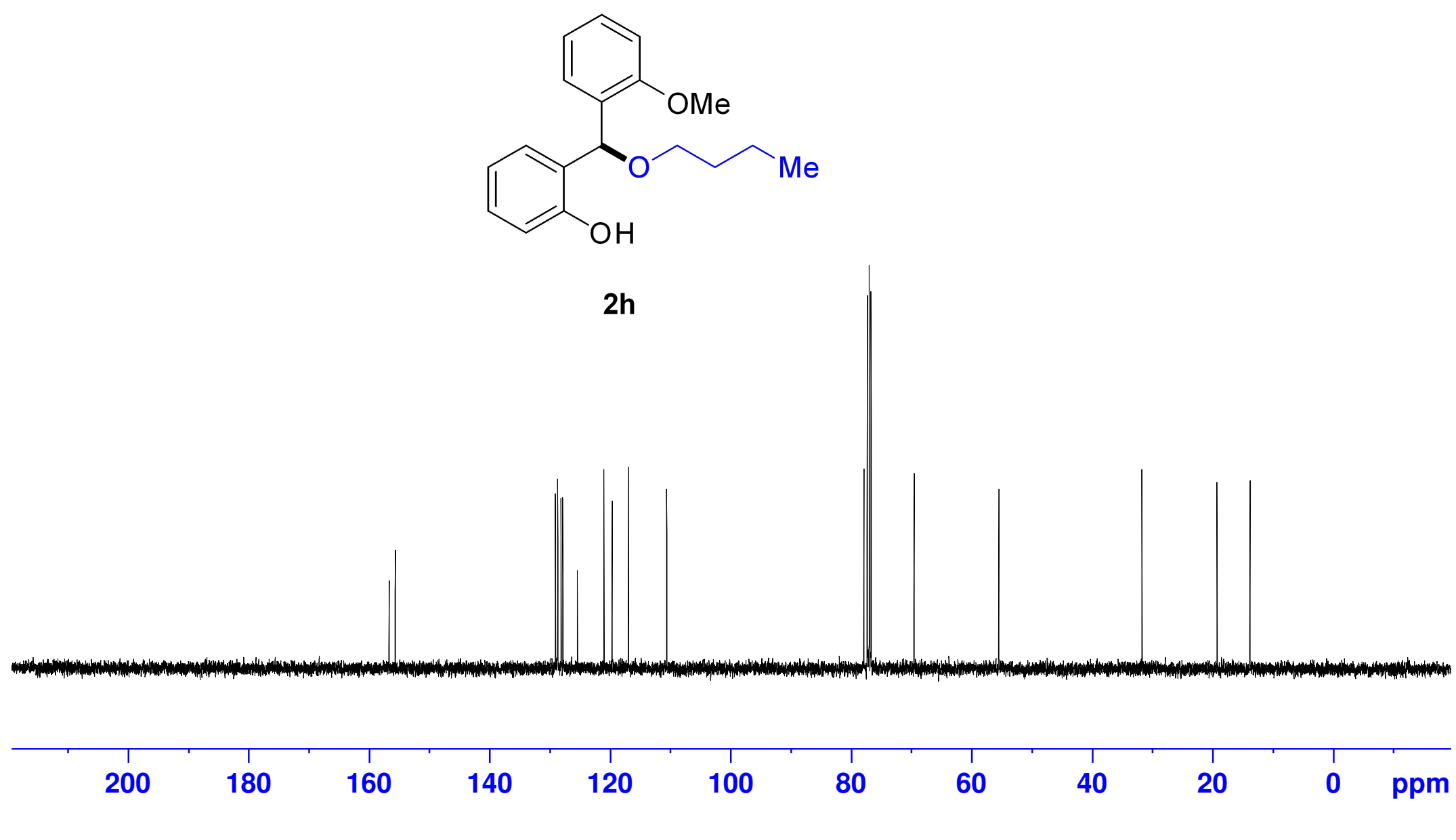

NAME

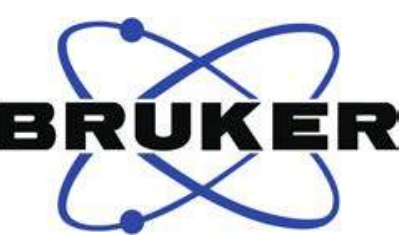

F2 - Acquisition Parameters

Date_ 20150512

$\begin{array}{lr}\text { Time } & 13.59\end{array}$

INSTRUM spect

PROBHD $5 \mathrm{~mm}$ PABBO BB/

PULPROG Zgpg30

$\begin{array}{ll}\text { TD } & 65536\end{array}$

SOLVENT

NS

SWH

FIDRES

FIDR
AQ
RG

DW

$\mathrm{DE}$

D1

D 11

$\mathrm{CDC}$
20
0

$24038.461 \mathrm{~Hz}$ $0.366798 \mathrm{~Hz}$

$1.3631488 \mathrm{sec}$ 196.92

20.800 usec 6.50 usec

$.0296 .9 \mathrm{~K}$

$2.00000000 \mathrm{sec}$

TD 0

$======$

$\mathrm{SFO1}$

$\mathrm{NUC1}$
$\mathrm{P} 1$

1

P1

CHANNEL $\mathrm{fl}=======$

PLW1

$100.6228298 \mathrm{MHz}$

9.70

9.70 use

$\mathrm{SFO} 2$
$\mathrm{NUC2}$

CPDPRG [ 2

PCPD2

LW2

LW12

NNEL $f 2$ $========$

$400.1316005 \mathrm{MHz}$

$1 \mathrm{H}$
waltzi6

11.99499989 W

$0.34213999 \mathrm{~W}$
$0.27713001 \mathrm{~W}$

2 - Processing parameters SI 32768

SF $\quad 100.6127773 \mathrm{MHz}$ 
œ 일

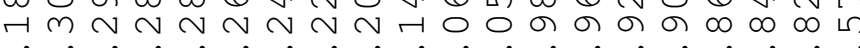

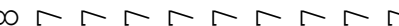
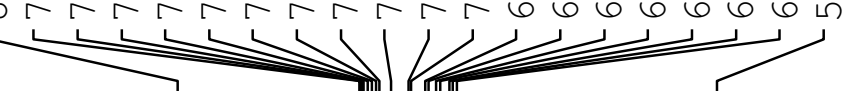

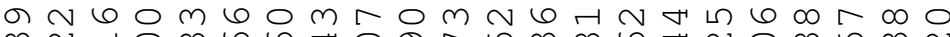

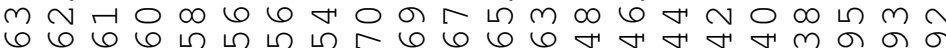
. . . . . . . . . . . mmmmmmm

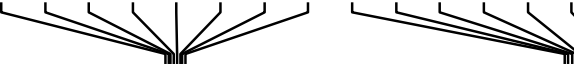

\section{ex uxer $(1)$}

Current Data Parameters NAME

PROCNO

l zw2180C-H

F2 - Acquisition Parameters

Date__ 20150514

Time $\quad 16.06$

$5 \mathrm{~mm}$ spect

$\mathrm{zg} 30$
65536

$\begin{array}{ll}\text { TD } & 65536 \\ \text { SOLVENT } & \text { CDC13 }\end{array}$

NS

SWH

FIDRES

$\mathrm{AQ}$

RG

DW

DE

D1

TD 0

$====$
$\mathrm{SFO} 1$

$\mathrm{NUC}$

P 1
PLW1

$\mathrm{CDCl} 3$
7
0

$8012.820 \mathrm{~Hz}$

$0.122266 \mathrm{~Hz}$

$4.0894465 \mathrm{sec}$ 45.67

62.400 usec 6.50 use

$1.00000000 \mathrm{sec}$

$400.1324710 \mathrm{MHz}$

14.50 usec

$11.99499989 \mathrm{~W}$

F2 - Processing parameters

SI 65536

SF $\quad 400.1300088 \mathrm{MHz}$

WSB

SSB

$\mathrm{EM}$

$0.30 \mathrm{~Hz}$

1.00

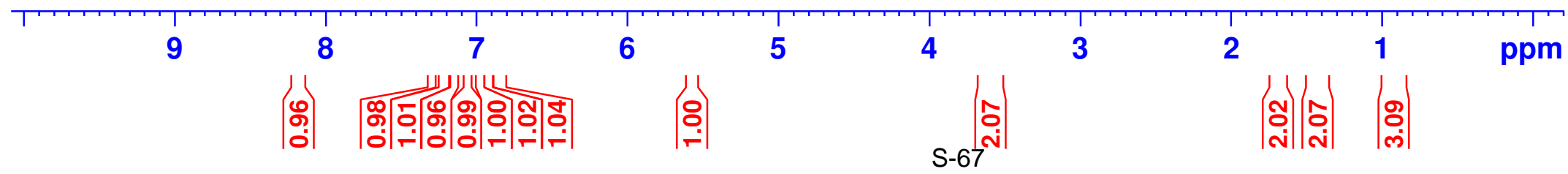



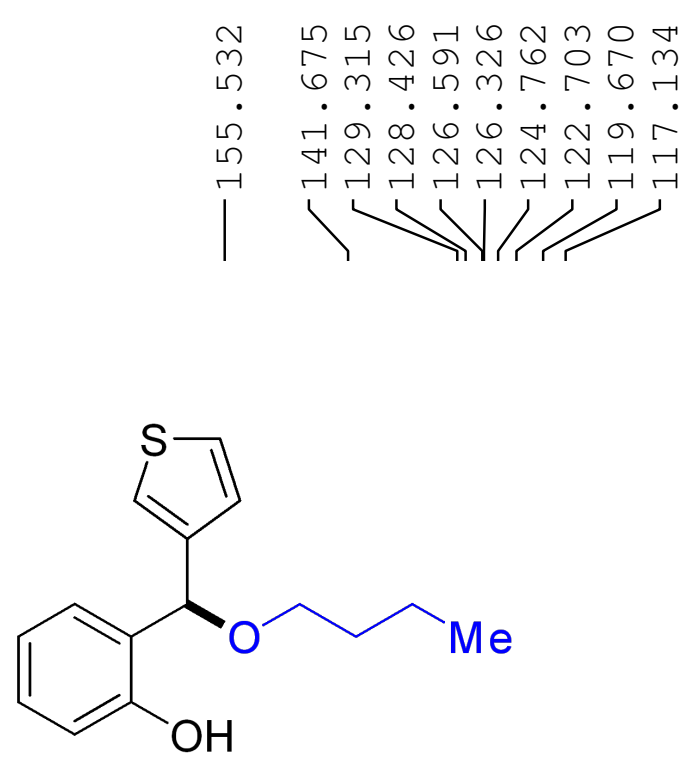

2i

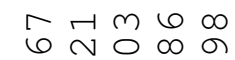

$\sim m 06 \%$

드응

111

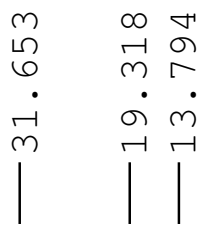

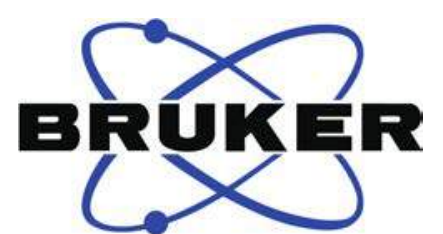

Current Data Parameters

NAME

l zw2180C-C

PROCNO

F2 - Acquisition Parameters

Date__ 20150514

16.09

Time

PROBHD

PULPROG zgpg30

$5 \mathrm{~mm} \mathrm{PABBO} \mathrm{BB/}$

TD

NS

DS

SWH

FIDRES

FIDR
AQ

AQ

DW

DE

TE

D 11

TD 0

$======$

$\mathrm{SFO} 1$
$\mathrm{NUC1}$

$\mathrm{NUC}$
$\mathrm{P} 1$

P 1
PLW1

zgpg 30
65536

$\mathrm{CDCl} 13$

$24038.461 \mathrm{~Hz}$

$24038.461 \mathrm{~Hz}$

$1.3631488 \mathrm{sec}$

196.92

20.800 usec

6.50 usec

$20.0 \mathrm{~K}$

$2.00000000 \mathrm{sec}$

$0.03000000 \mathrm{sec}$

CHANNET 11 = $==2=$

$100.6228298 \mathrm{MHz}$ $13 \mathrm{C}$ $\begin{aligned} & 9.70 \text { usec } \\ & 46.98899841 \mathrm{~W}\end{aligned}$ $========$ CHANNEL $\mathrm{f} 2========$

NUC2

CPDPRG [2

PCPD2

PLW12

PLW13

$400.1316005 \mathrm{MHz}$

$1 \mathrm{H}$

90.00 usec

$11.99499989 \mathrm{~W}$ $0.34213999 \mathrm{~W}$

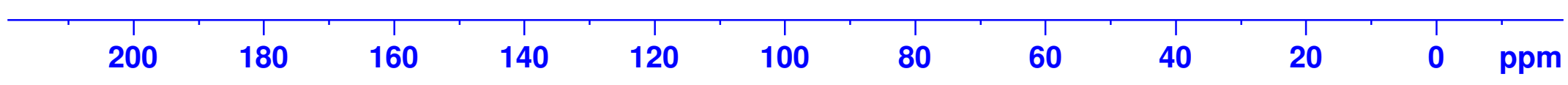

F - Processing parameters SI $\quad 32768$

(2)




\section{aporen $(1)$}

Current Data Parameters NAME

PROCNO

lzw2150A-H

F2 - Acquisition Parameters

Date 20150429

Time

Time

PROBHD

PULPROG $\quad$ zg30
P

$5 \mathrm{~mm} \mathrm{PABBO} \mathrm{BB} /$

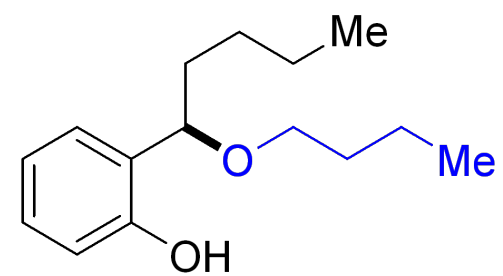

SOLVENT

NS

DWH

FIDRES

$\mathrm{AQ}$

$\mathrm{RG}$

DW

$\mathrm{DE}$

2j
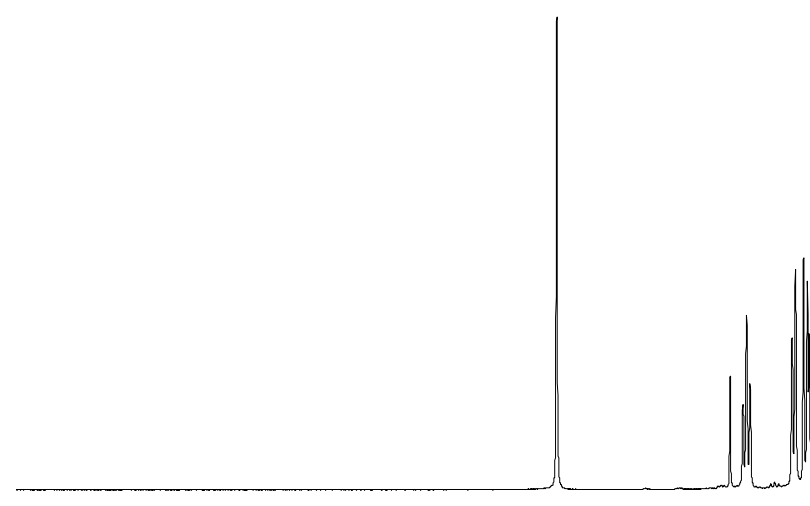

\begin{abstract}
8
\end{abstract}

7

|น้ำ

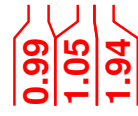

6

5

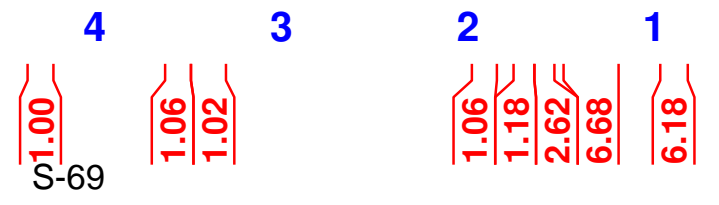




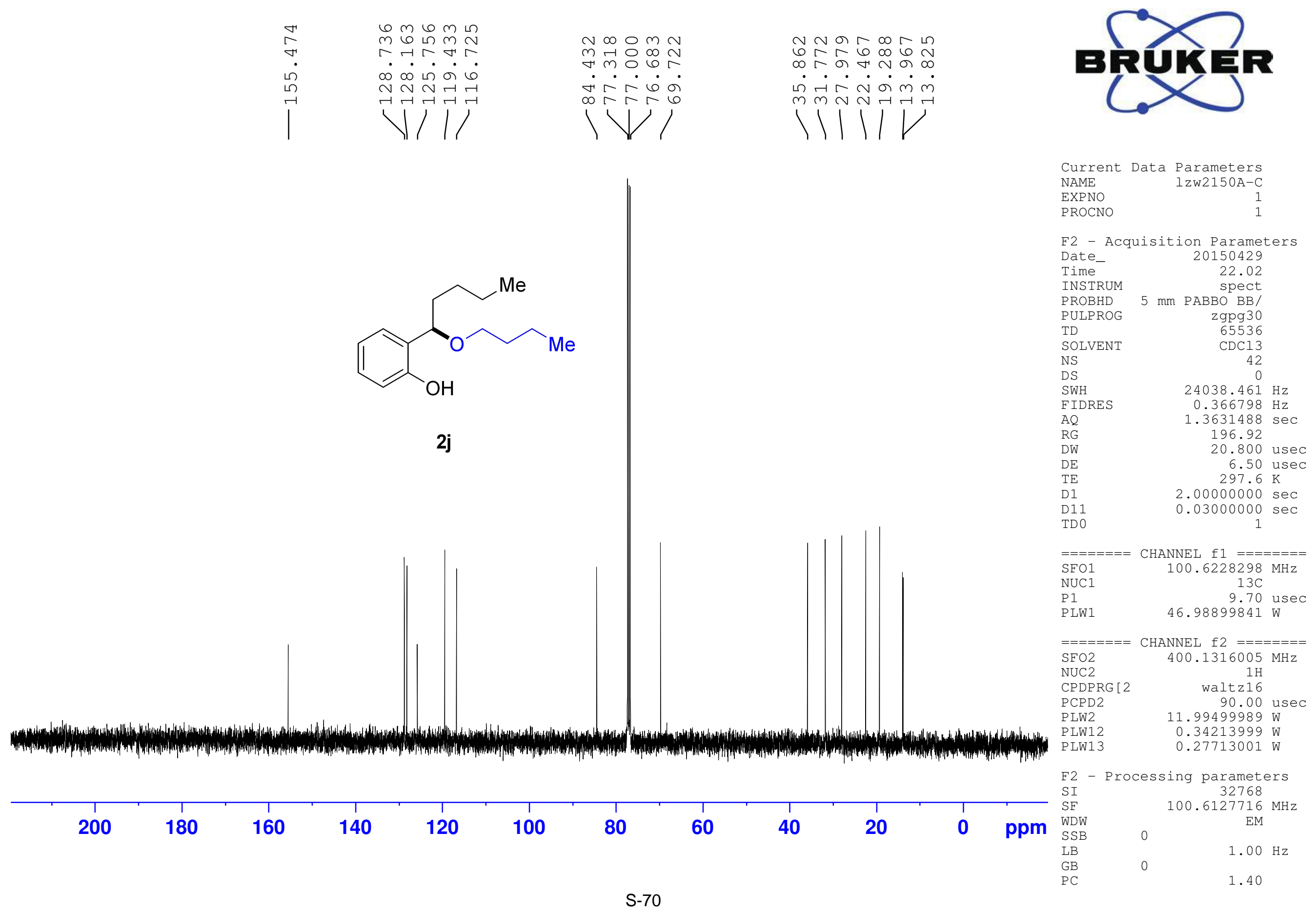


Current Data Parameters NAME

EXPNO
PROCNO

lzw3065-H

F2 - Acquisition Parameters

Date__ 20150629

20150629

spect
INSTRUM

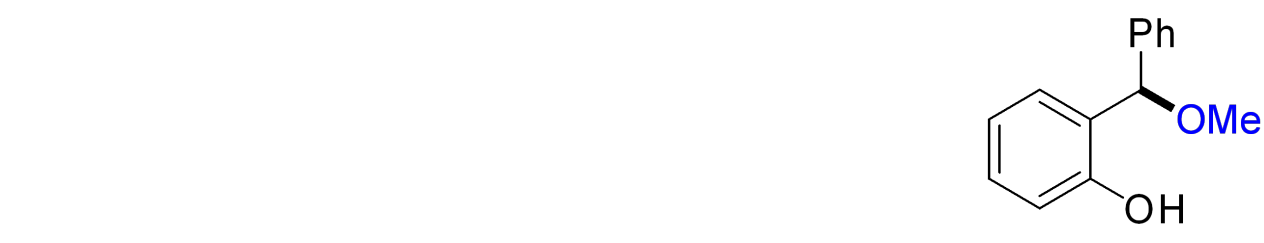

PULPROG

$\mathrm{mm} \mathrm{PABBO} \mathrm{BB} /$

$\begin{array}{lr}\text { TD } & \text { zg30 } \\ & 65536\end{array}$

SOLVENT

NS

SWH

FIDRES

$\mathrm{AQ}$

$2 k$

SI $\quad 65536$

SF $\quad 400.1300091 \mathrm{MHz}$

WSW

LB

EM

$0.30 \mathrm{~Hz}$

GB

1.00

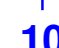




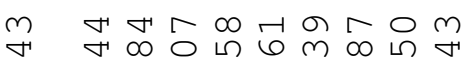

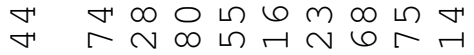

$\dot{\sigma} \dot{\sigma} \dot{\sigma} \dot{\infty} \dot{\infty} \dot{\sim} \dot{\sigma} \dot{\sim}$

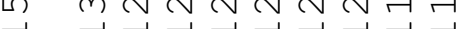

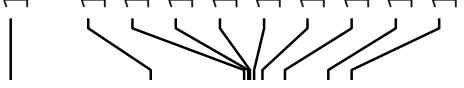

당 न

เก $m a$

$6 \circ 6$<smiles>COC(c1ccccc1)c1ccccc1O</smiles>

$2 k$

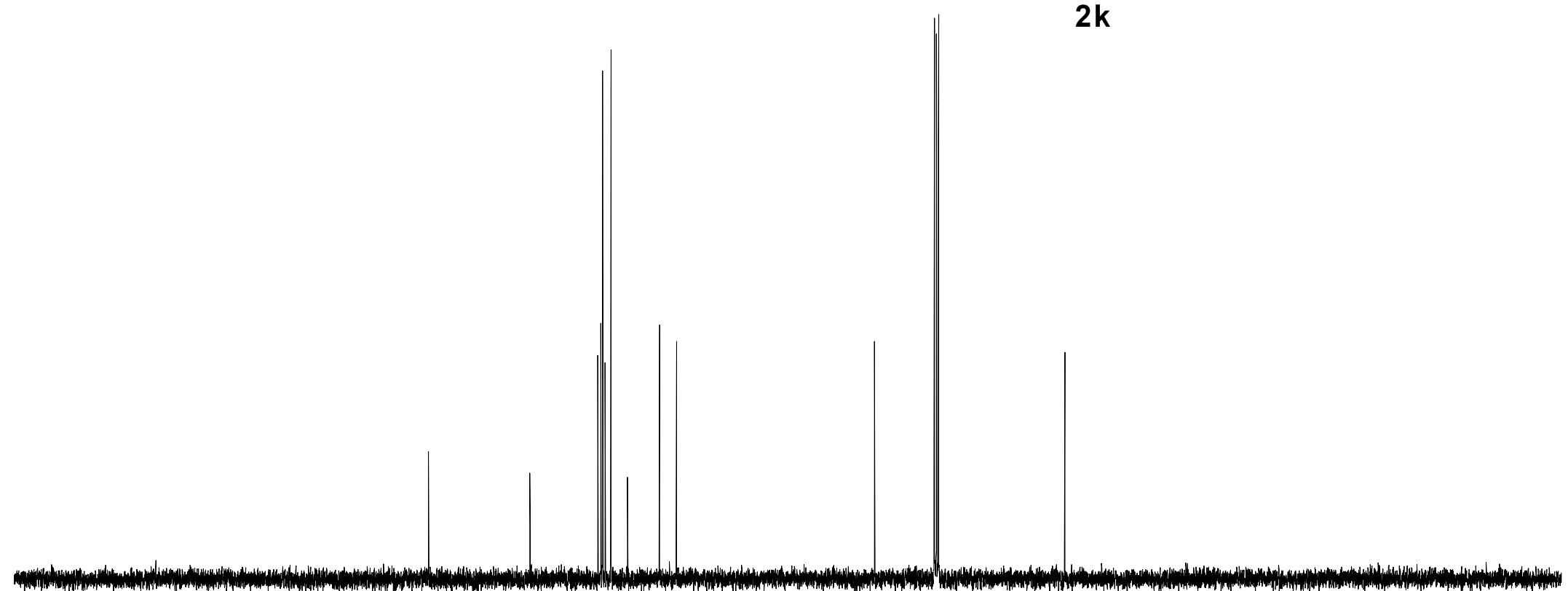

200 
$\infty \begin{aligned} 4 \pi \\ \infty\end{aligned}$ m $m$ m

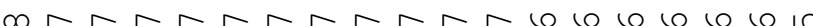
$\longrightarrow$ $\infty \circ \leqslant \infty$ ก 구의 $\dot{m} \dot{m} \dot{m} \dot{m} \dot{m} \dot{m} \dot{m} \dot{m}$ $\longrightarrow$
เ) $m m$ r $+\frac{1}{1+1}$<smiles>CCOC(c1ccccc1)c1ccccc1O</smiles>

2I

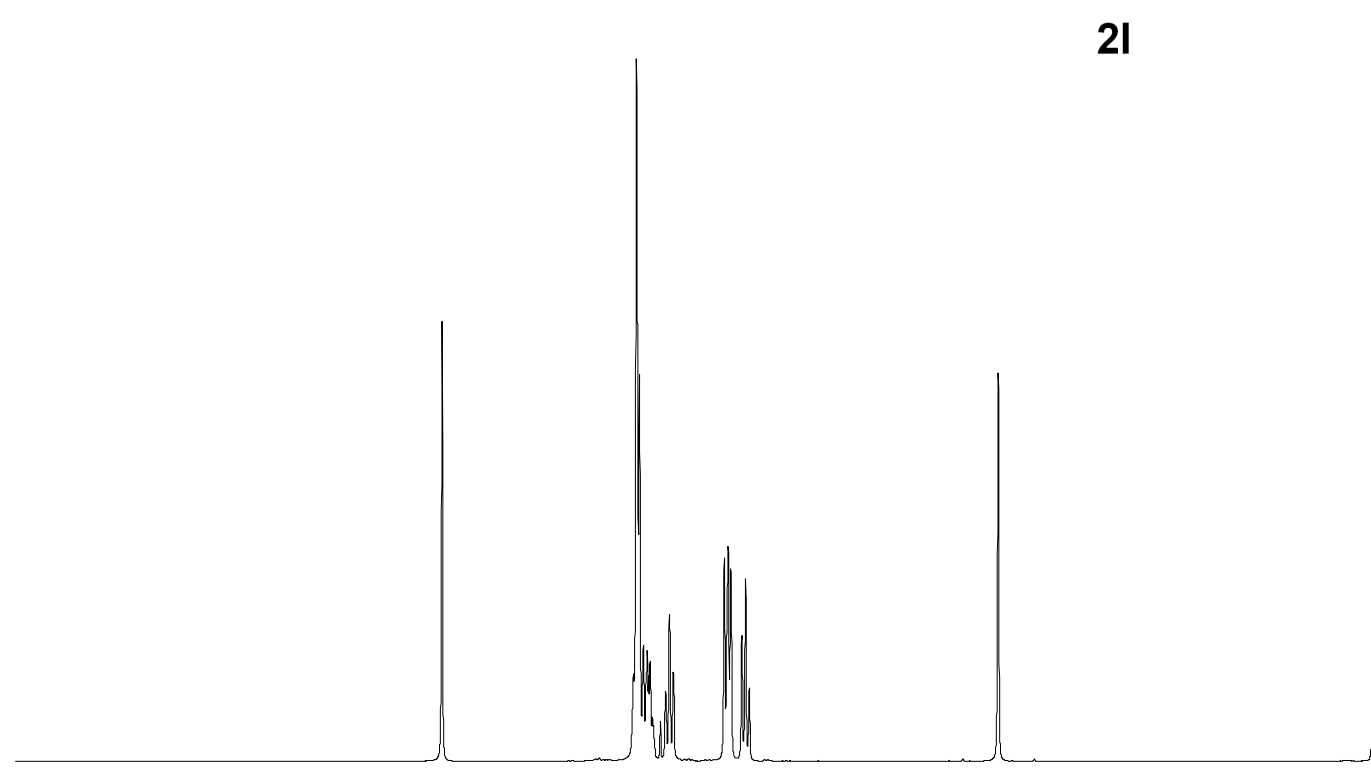

\section{aporen $(1)$}

Current Data Parameters NAME lzw3030D-H$$
\text { PROCNO }
$$

F2 - Acquisition Parameters

Date 20150611

$\begin{array}{lr}\text { Time } & 13.22\end{array}$

SPect

$\mathrm{zg} 30$
65536

$\begin{array}{ll}\text { TD } & 65536 \\ \text { SOLVENT } & \text { CDC13 }\end{array}$

NS

DS

SWH

$\mathrm{AQ}$

RG

DW

DE

DD 0

$====$

$\mathrm{SFO}$

$\mathrm{NUC}$

$\mathrm{CDC} 13$
9

\section{$8012.820 \mathrm{~Hz}$}

$0.122266 \mathrm{~Hz}$

$4.0894465 \mathrm{sec}$ 39.46

62.400 usec 6.50 usec

$1.00000000 \mathrm{sec}$ 1

CHANNEL $\mathrm{f} 1 \mathrm{l}========$ $400.1324710 \mathrm{MHz}$ 4.50 usec PLW1 11.99499989 W

F2 - Processing parameters

SI 65536

SF $\quad 400.1300103 \mathrm{MHz}$

SSB

EM

$0.30 \mathrm{~Hz}$

1.00 

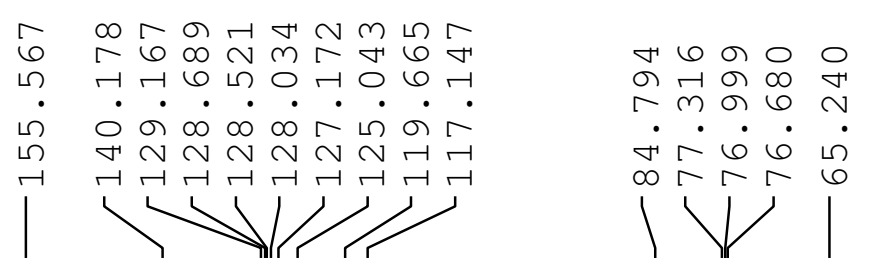

\section{BR $(1)$}

Current Data Parameters NAME

EXPNO

lzw3030D-C<smiles>CCO[C@@H](c1ccccc1)c1ccccc1O</smiles>

F2 - Acquisition Parameters Date__ 20150611

$\begin{array}{ll}\text { Time } & 13.26\end{array}$

Spect

$\begin{array}{lr}\text { PULPROG } & \text { zgpg30 } \\ \text { TD } & 6536\end{array}$

$\begin{array}{lr}\text { TD } & 65536 \\ \text { SOLVENT } & \text { CDC13 }\end{array}$

DS

2 I

$\begin{array}{lr}\text { SWH } & 24038.461 \mathrm{~Hz} \\ \text { FIDRES } & 0.366798 \mathrm{~Hz}\end{array}$ $0.366798 \mathrm{~Hz}$
$1.3631488 \mathrm{sec}$ 196.92 6.50 usec
297.5 Kasec $297.5 \mathrm{~K}$

$2.00000000 \mathrm{sec}$

$=======$ CHA

SFO1 $100.6228298 \mathrm{MHz}$ NUC1

NUC

P 1
PLW1 $13 \mathrm{C}$

$46.98899841 \mathrm{~W}$

$========$ CHANNEL $\mathrm{f} 2========$ SFO2 $400.1316005 \mathrm{MHz}$

NUC2

CPDPRG [ 2

PCPD2

PLW2

PLW12
PLW13

$1 \mathrm{H}$

90.00 usec

$11.99499989 \mathrm{~W}$

$0.34213999 \mathrm{~W}$ $0.27713001 \mathrm{~W}$

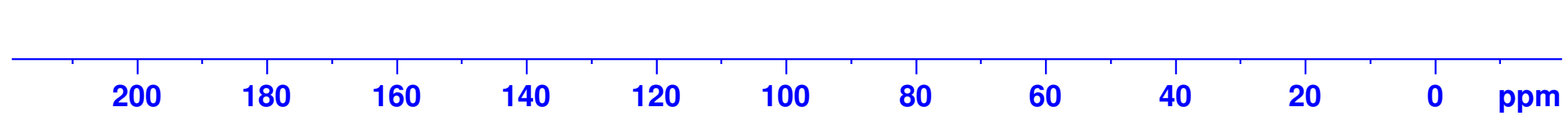

F2 - Processing parameters SI 32768

SF $\quad 100.6127780 \mathrm{MHz}$

80

60




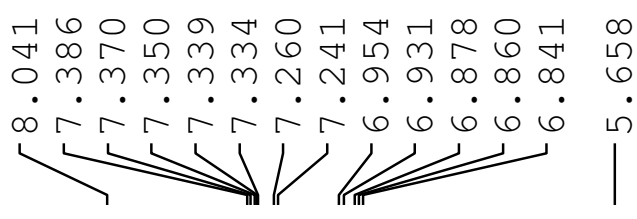

$\begin{array}{llll}0 & -1 & m & r \\ 0 & 1 & m & 0 \\ 6 & 6 & 6 & 6\end{array}$

में $\dot{\gamma} \dot{\gamma}$

में<smiles>Oc1ccccc1C(Cc1ccccc1)c1ccccc1</smiles>

$2 m$

\section{ex $(->)$}

Current Data Parameters NAME

PROCNO

l $\mathrm{zW} 2109 \mathrm{~B}-\mathrm{H}$

F2 - Acquisition Parameters

Date_ 20150409

$\begin{array}{lr}\text { Time } & 22.23\end{array}$

INSTRUM spect

PULPROG

zg30
65536

SOLVENT

NS

DS

SWH

IDRES

$\mathrm{AQ}$

RG

$\mathrm{DW}$
$\mathrm{DE}$
$\mathrm{TE}$

TE

TD 0

$===$

$\mathrm{SFO1}$

$\mathrm{NUC}$

P1
PLW1

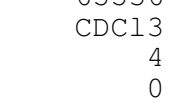

$8012.820 \mathrm{~Hz}$

$0.122266 \mathrm{~Hz}$

$4.0894465 \mathrm{sec}$

70.97

62.400 usec 6.50 usec

$1.00000000 \mathrm{sec}$ 1

CHANNEL $\mathrm{fl}========$ $400.1324710 \mathrm{MHz}$

14.50 $11.99499989 \mathrm{~W}$

F2 - Processing parameters

SI 65536

$\begin{array}{lll}\text { SF } & 400.1300092 \mathrm{MHz}\end{array}$

WDW

SSB

EM

$0.30 \mathrm{~Hz}$

1.00

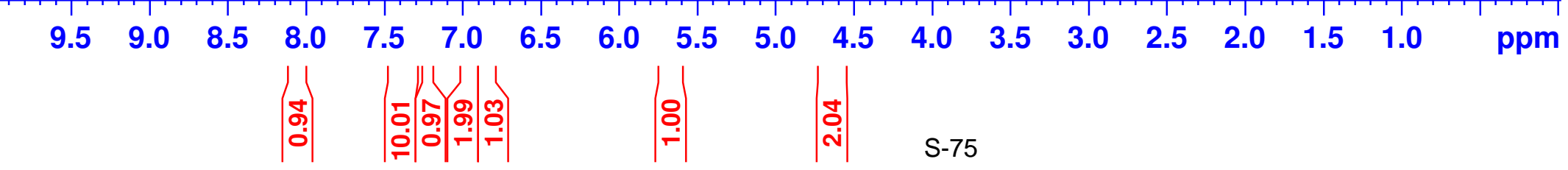




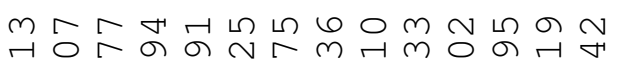

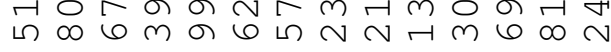

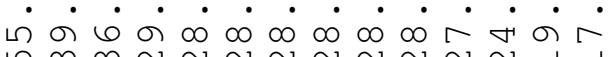

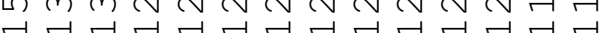

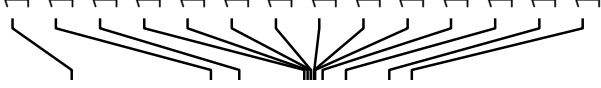

$\infty \circ m i m$ $\forall m 06 r$ $\dot{m} \wedge \dot{r} \dot{r}$ IVI

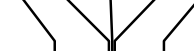<smiles>Oc1ccccc1C(OCc1ccccc1)c1ccccc1</smiles>

$2 m$

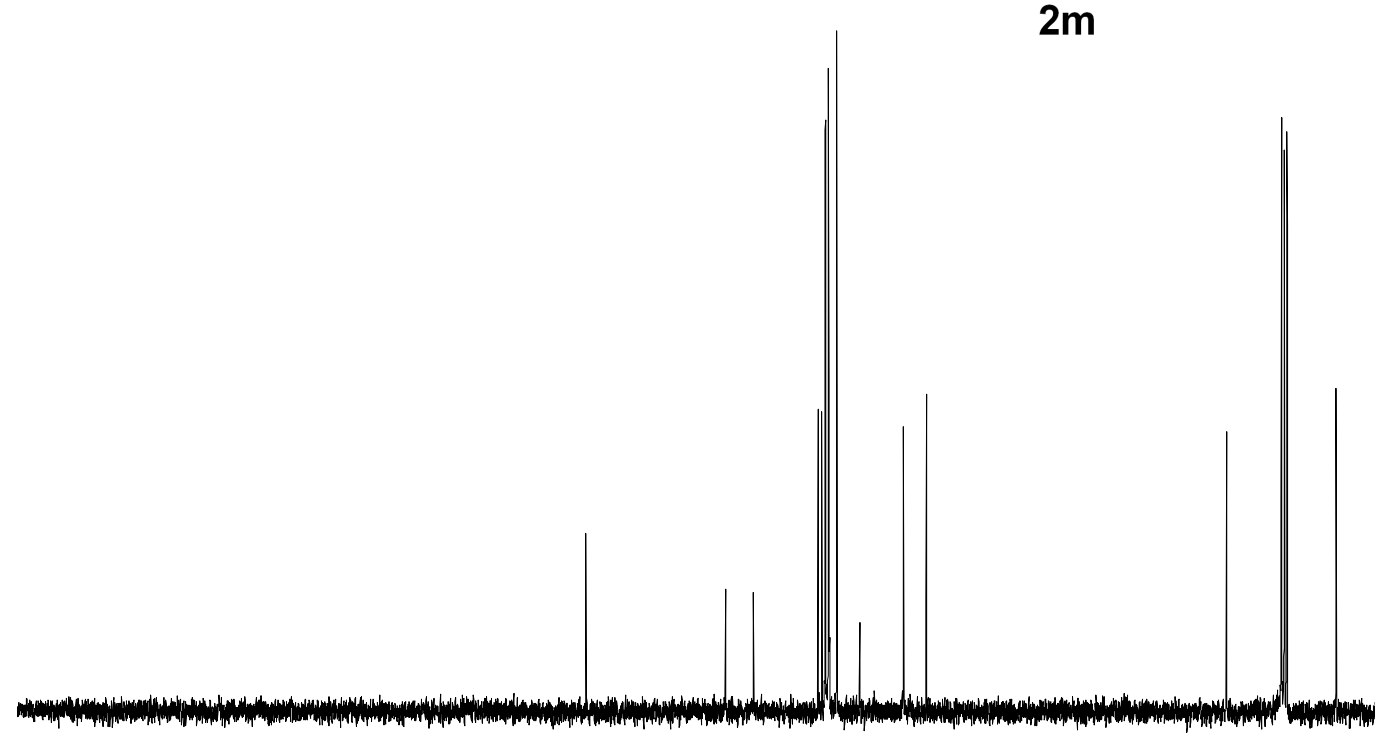

80

40




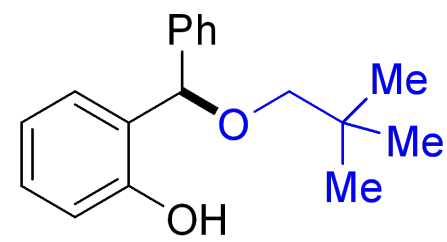

$2 n$
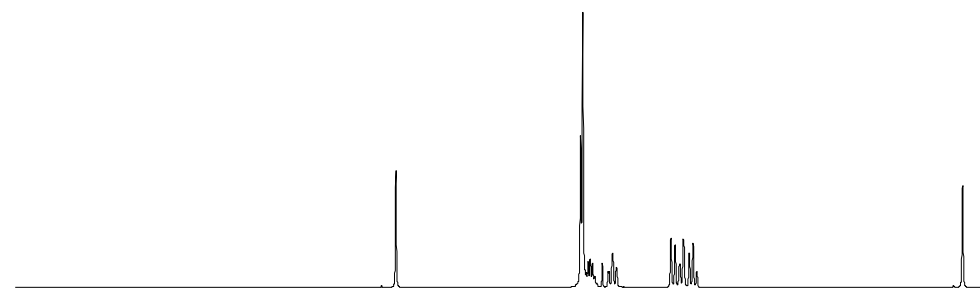


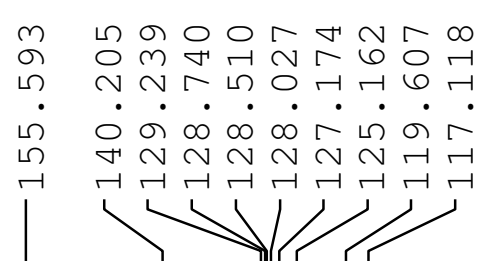

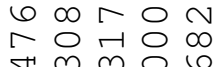

r m mo 6

ம $0 \therefore \approx 6$

111 $\circ$
$\infty$
$\circ$

- $\dot{0}$

1

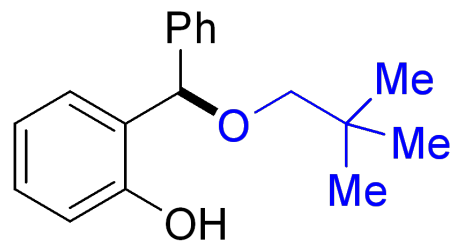

$2 n$

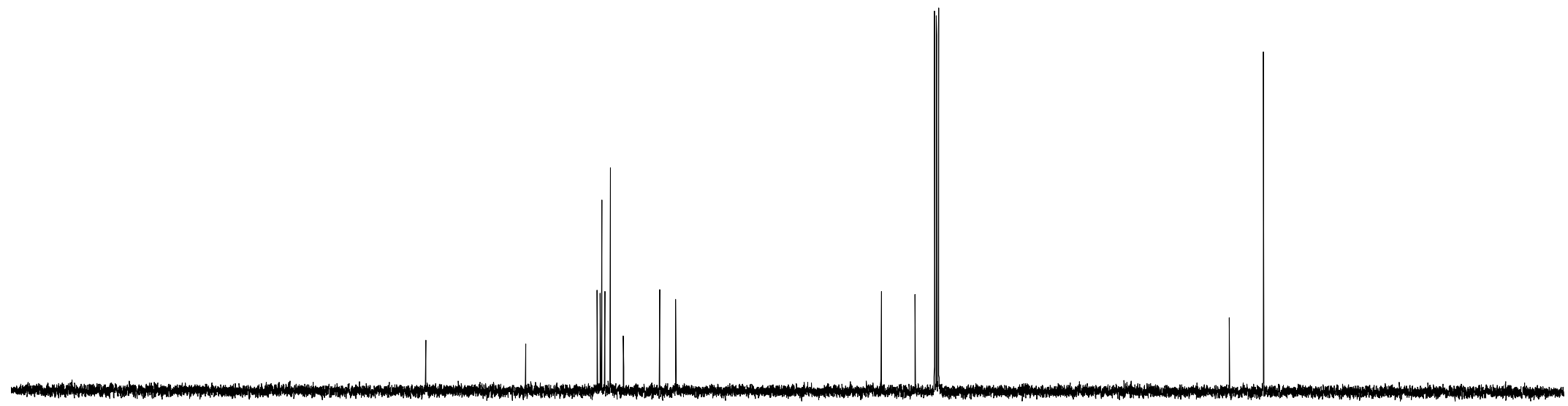

80 


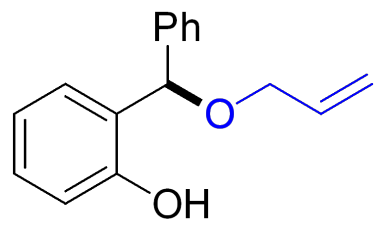

20
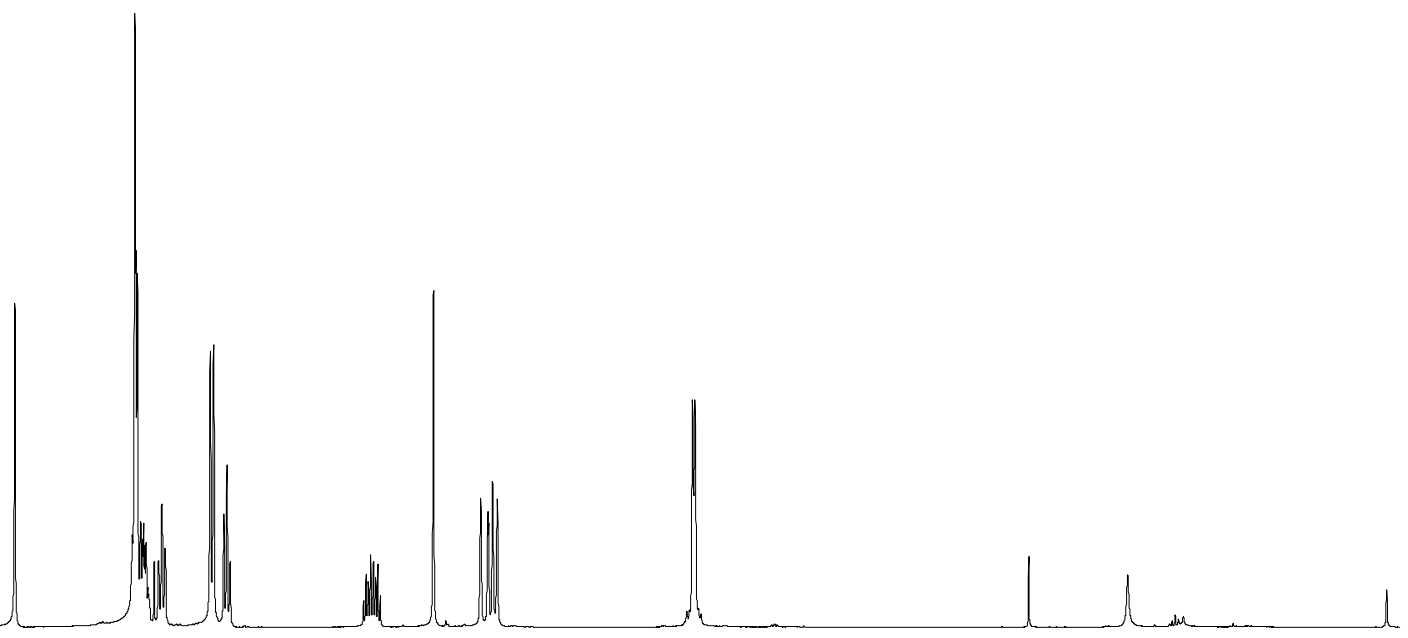

\section{en $(>$}

Current Data Parameters NAME

PROCNO

lzw2109A-H

F2 - Acquisition Parameters

Date_ 20150408

Time 11.39

$5 \mathrm{spect}$

PROBHD

zg30
65536

SOLVENT

NS

DS

SWH

IDR

$\mathrm{AQ}$

RG

DW

$\mathrm{DE}$

D1
TD 0

$====$

$\mathrm{SFO1}$

$\mathrm{NUC1}$
$\mathrm{P} 1$

65536

7

\section{$8012.820 \mathrm{~Hz}$}

$0.122266 \mathrm{~Hz}$

$4.0894465 \mathrm{sec}$ 62.93

62.400 usec 6.50 use

$1.00000000 \mathrm{sec}$

$$
1
$$

CHANNEL $\mathrm{f} 1 \mathrm{l}========$ $400.1324710 \mathrm{MHz}$

14.50

usec

PLW1 $11.99499989 \mathrm{~W}$

F2 - Processing parameters

SI $\quad 65536$

$\begin{array}{ll}\text { SF } & 400.1300093 \mathrm{MHz}\end{array}$

TSB

EM

$0.30 \mathrm{~Hz}$

1.00

4

3




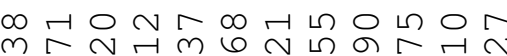

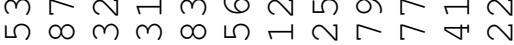

ம் $\dot{n} \dot{m} \dot{\sigma} \dot{\infty} \dot{\infty} \dot{\sim} \dot{\sim} \dot{\sigma} \dot{\circ}$

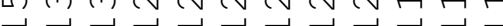

>

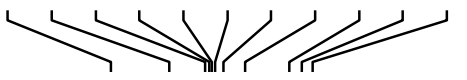

$\circ \cap \sim m \infty$

$\therefore$ 다의 00

$\dot{m} \dot{\sim} \dot{\sim} \dot{0}$

111

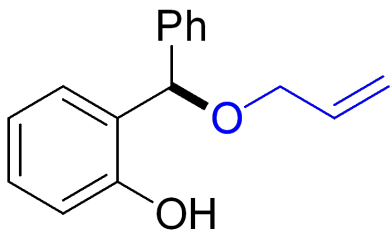

20

\section{ef $(>$}

Current Data Parameters NAME

EXPNO

lzw2109A-C

F2 - Acquisition Parameters

Date

20150408

Time

INSTRUM

PULPROG

14.06

$5 \mathrm{~mm}$ PABBO BB/

zgpg 30

TD 65536

SOLVENT

NS

SWH

FIDRES

$\mathrm{AQ}$
$\mathrm{RG}$

DW

$\mathrm{DE}$

TE

D1 1

$\mathrm{CDCl3}$

144

$24038.461 \mathrm{~Hz}$ $0.366798 \mathrm{~Hz}$

$1.3631488 \mathrm{sec}$ 196.92

20.800 usec 6.50 usec $0007.5 \mathrm{~K}$ $2.00000000 \mathrm{sec}$ 0.03000000 s

$======$

$\mathrm{SFO1}$

P 1

P1
PLW1

CHANNEL $\mathrm{f} 1========$ $100.6228298 \mathrm{MHz}$ $13 \mathrm{C}$ $\begin{aligned} & 9.70 \text { usec } \\ & 46.98899841 \mathrm{~W}\end{aligned}$

$========$ CHANNEL f2 $========$ $\mathrm{SFO} 2$

NUC2

CPDPRG [2

PCPD2

PLW2

PLW12

PLW13

$400.1316005 \mathrm{MHz}$

$1 \mathrm{H}$

waltz16

$11.99499989 \mathrm{~W}$

$0.34213999 \mathrm{~W}$ $0.27713001 \mathrm{~W}$

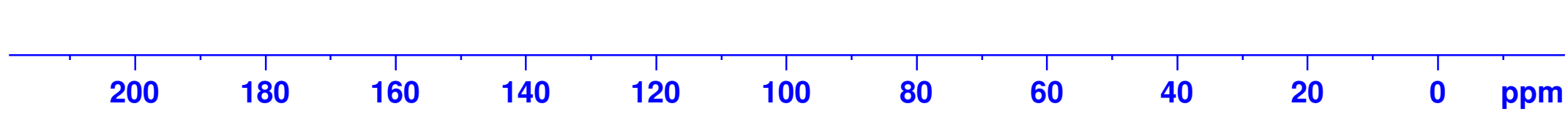

2 - Processing parameters SI 32768 SF $\quad 100.6127748 \mathrm{MHz}$

\begin{abstract}
120
\end{abstract}




\section{BR $(->1)$}

Current Data Parameters NAME Izw3027C-H

PROCNO

F2 - Acquisition Parameters

Date_ 20150606

Time 20150606
16.49<smiles>C=C(C)CO[C@H](c1ccccc1)c1ccccc1O</smiles>

$2 p$

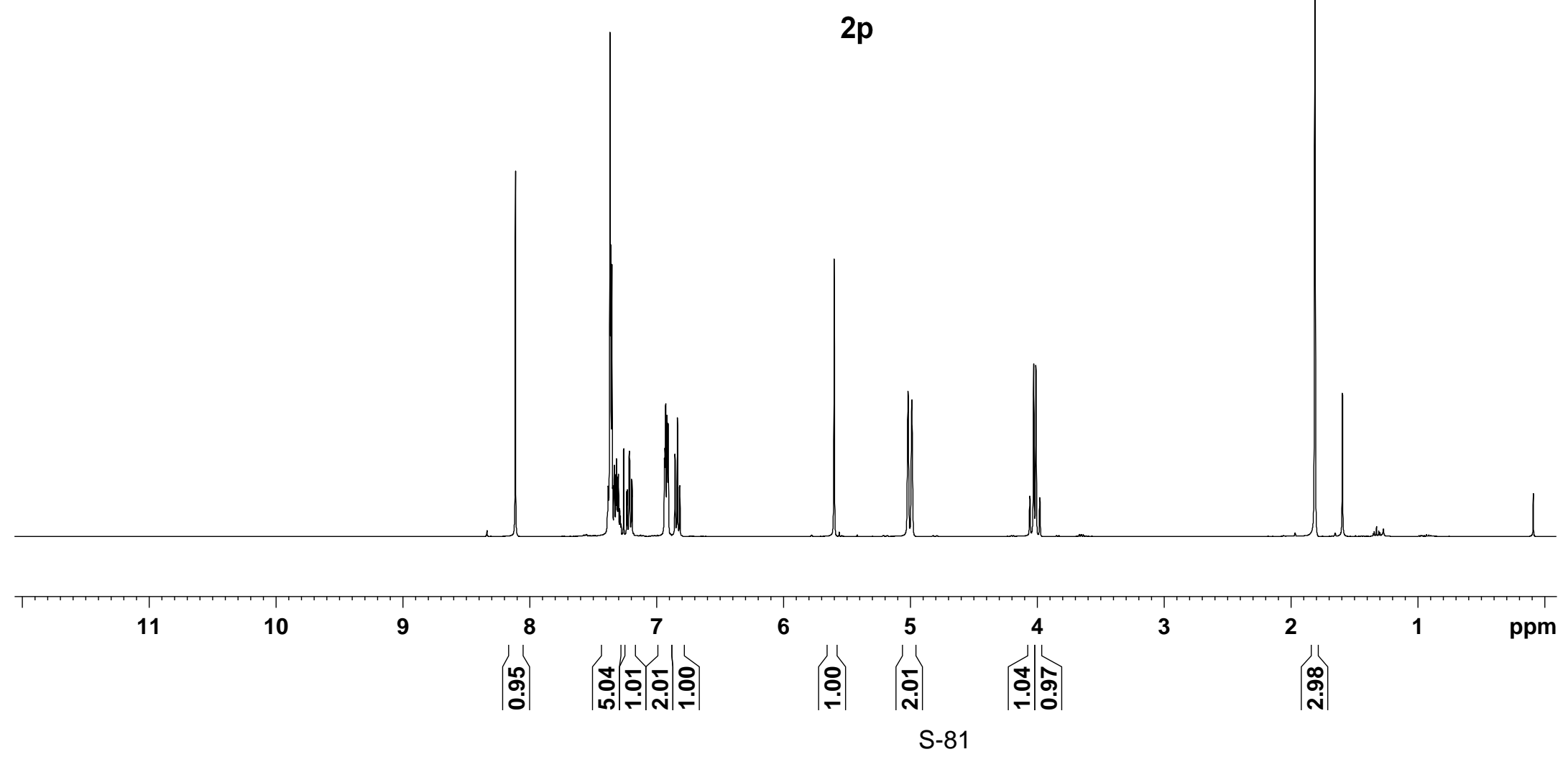

spect
PROBHD $5 \mathrm{~mm}$ PABBO BB-

PULPROG Zg30

TD 65536

SOLVENT

CDCl3

NS

SW

$\quad 8223.685 \mathrm{~Hz}$

FIDRES $\quad 0.125483 \mathrm{~Hz}$

$A Q$

DE $\quad 6.00$ usec

$1.00000000 \mathrm{sec}$

TDO 1

NUC1 $1 \mathrm{H}$

P1 13.60 usec

$\begin{array}{lc}\text { PL1 } & -1.00 \mathrm{~dB} \\ \text { SFO1 } & 400.1324710 \mathrm{MHz}\end{array}$

F2 - Processing parameters

SF

WDW

SSB

LB

EM

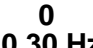

$0.30 \mathrm{~Hz}$
DW $\quad 60.800$ usec

$=======$ CHANNEL $\mathrm{f} 1 \mathrm{=}===$

$\begin{array}{lc}\text { SI } & 32768 \\ \text { SF } & 400.1300052 \mathrm{MHz}\end{array}$ 
Current Data Parameters

NAME Izw3027C-C

NAME

PROCNO

1

F2 - Acquisition Parameters

Date__ 20150606

Time

16.56

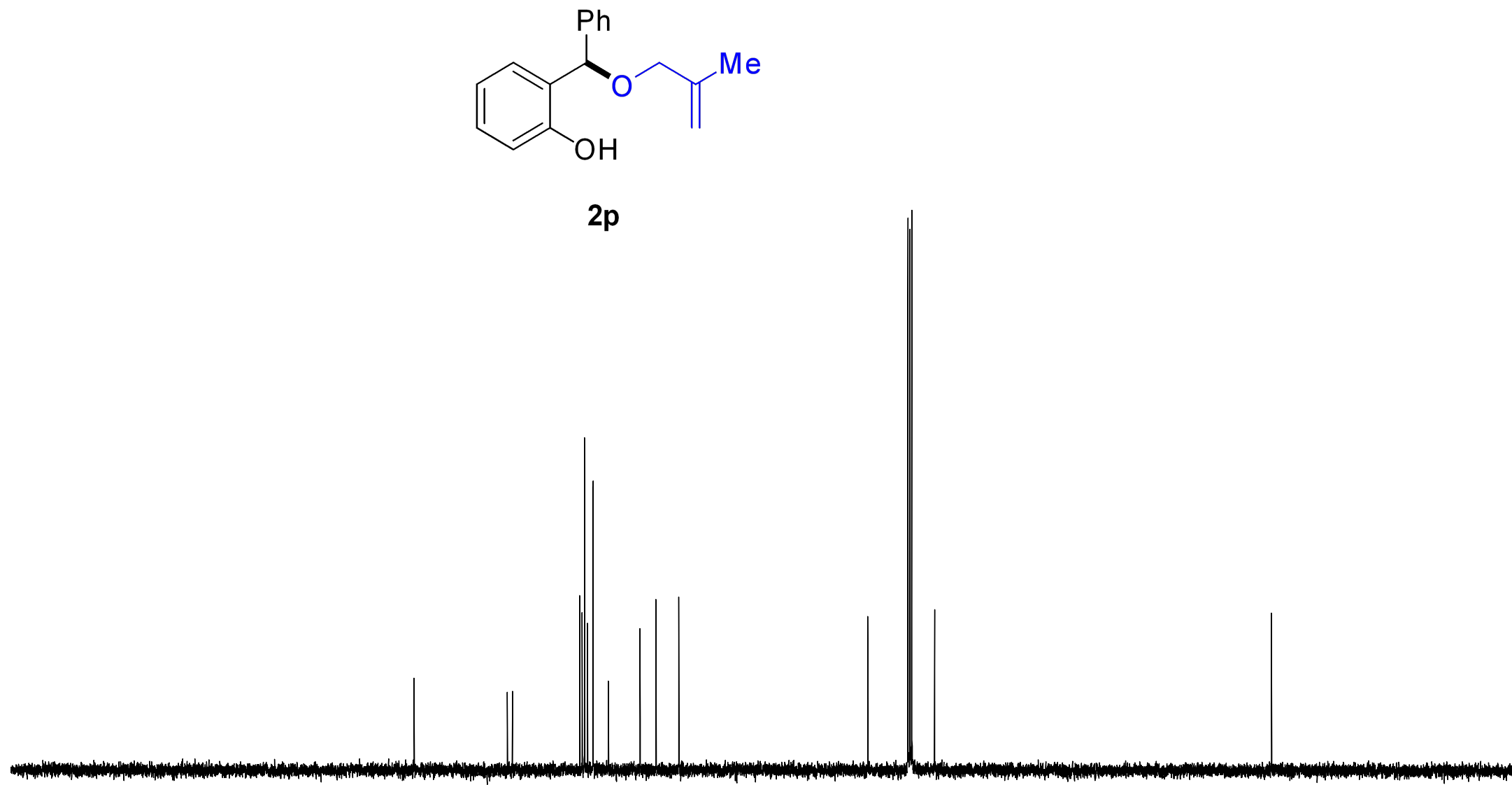

INSTRUM

spect

P 5 mm PABBO BB-

PULPROG zgpg30

TD $\quad{ }^{65536}$

NS

$\begin{array}{ll}\text { DS } & 2 \\ \text { SWH } & 24038.461 \mathrm{~Hz}\end{array}$

FIDRES $\quad 0.366798 \mathrm{~Hz}$

AQ $1.3631988 \mathrm{sec}$

RG 2050

20.800 usec

DE $\quad 6.00$ use

$\begin{array}{ll}\text { TE } & 294.0 \mathrm{~K} \\ \text { D1 } & 2.00000000 \mathrm{sec}\end{array}$

$2.00000000 \mathrm{sec}$
D11

$\begin{array}{ll}\text { d11 } & 0.03000000 \mathrm{sec} \\ \text { DELTA } & 1.89999998 \mathrm{sec}\end{array}$

TDO

\begin{tabular}{|c|c|}
\hline $\begin{array}{l}\text { NUC1 } \\
\text { P1 } \\
\text { PL1 } \\
\text { SFO1 }\end{array}$ & $\begin{array}{l}13 \mathrm{C} \\
9.25 \mathrm{usec} \\
-3.00 \mathrm{~dB} \\
100.6228298 \mathrm{MHz}\end{array}$ \\
\hline $\begin{array}{l}\text { :=:= } \\
\text { CPDPI } \\
\text { NUC2 } \\
\text { PCPD } \\
\text { PL12 } \\
\text { PL13 } \\
\text { PL2 } \\
\text { SFO2 }\end{array}$ & $\begin{array}{c}=\text { CHANNEL f2 ===== } \\
\text { waltz16 } \\
1 \mathrm{H} \\
80.00 \text { usec } \\
12.45 \mathrm{~dB} \\
18.00 \mathrm{~dB} \\
-1.00 \mathrm{~dB} \\
400.1316005 \mathrm{MHz}\end{array}$ \\
\hline $\begin{array}{l}\text { F2 - P } \\
\text { SI } \\
\text { SF } \\
\text { WDW } \\
\text { SSB } \\
\text { LB } \\
\text { GB } \\
\text { PC }\end{array}$ & $\begin{array}{c}\text { cessing parameters } \\
32768 \\
100.6127732 \mathrm{MHz} \\
\mathrm{EM} \\
0 \\
1.00 \mathrm{~Hz} \\
0 \\
1.40\end{array}$ \\
\hline
\end{tabular}

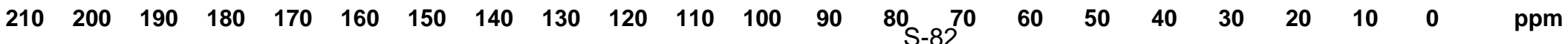




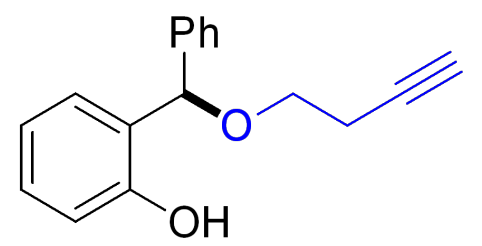

$2 q$
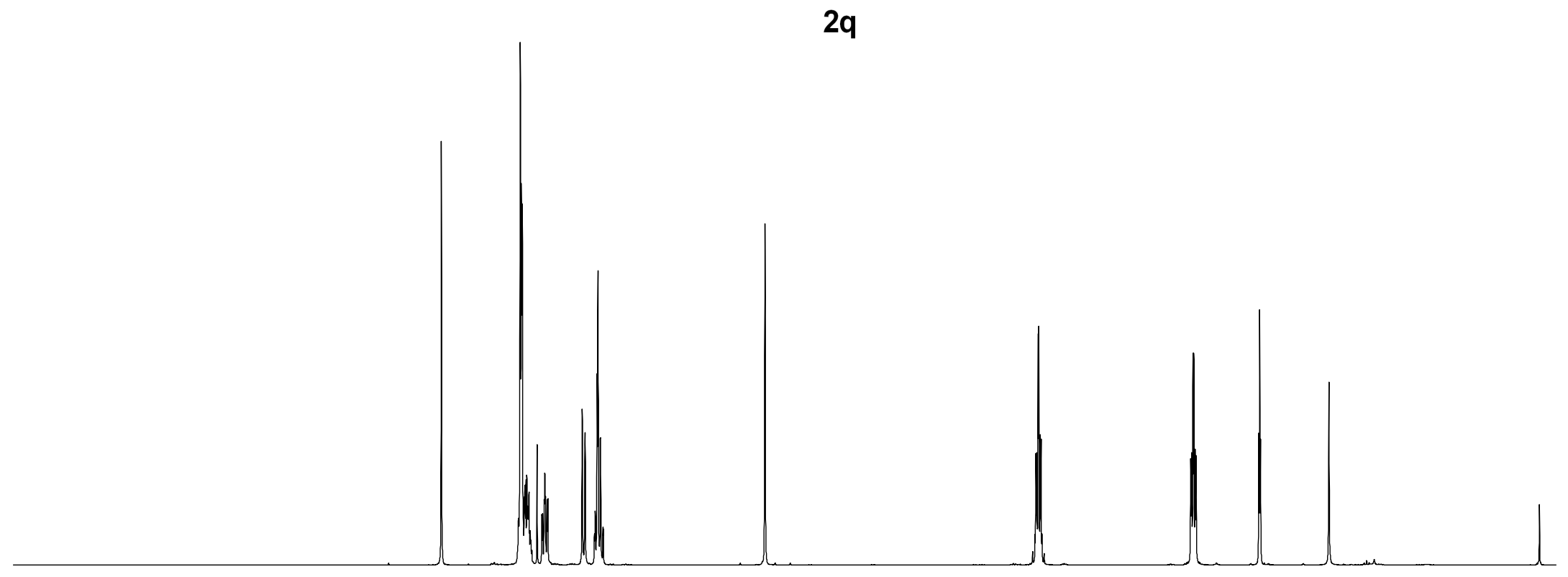

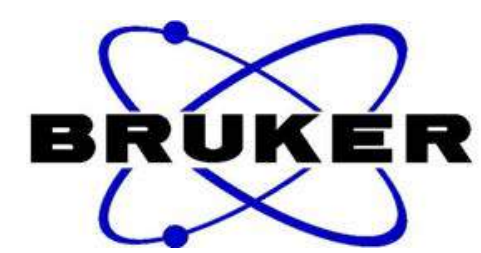

Current Data Parameters NAME Izw3027D-H

EXPNO

EXPNO

F2 - Acquisition Parameters

Date_ 20150606

Time

20.40

spect
PROBHD $5 \mathrm{~mm}$ PABBO

PULPROG

$\mathrm{zg} 30$

65536

SOLVENT

NS

SWH

$8223.685 \mathrm{~Hz}$

FIDRES $\quad 0.125483 \mathrm{~Hz}$

$.9846387 \mathrm{sec}$

RG 256

DW 60.800 usec

DE $\quad 6.00$ usec

TE $\quad 1.0000 .2 \mathrm{~K}$

TDO 1.000000

$======$ CHANNEL $\mathrm{f} 1 \mathrm{=}===$

P1 13.60 usec

PL1 $\quad \mathbf{- 1 . 0 0 ~ d B}$
SFO1 $\quad 400.1324710 \mathrm{MH}$

F2 - Processing parameters

SI 32768

$400.1300052 \mathrm{MHz}$

WDW

SSB

LB

EM

$\stackrel{0}{0.30} \mathrm{~Hz}$

0 


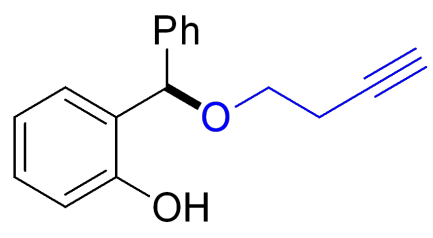

$2 q$

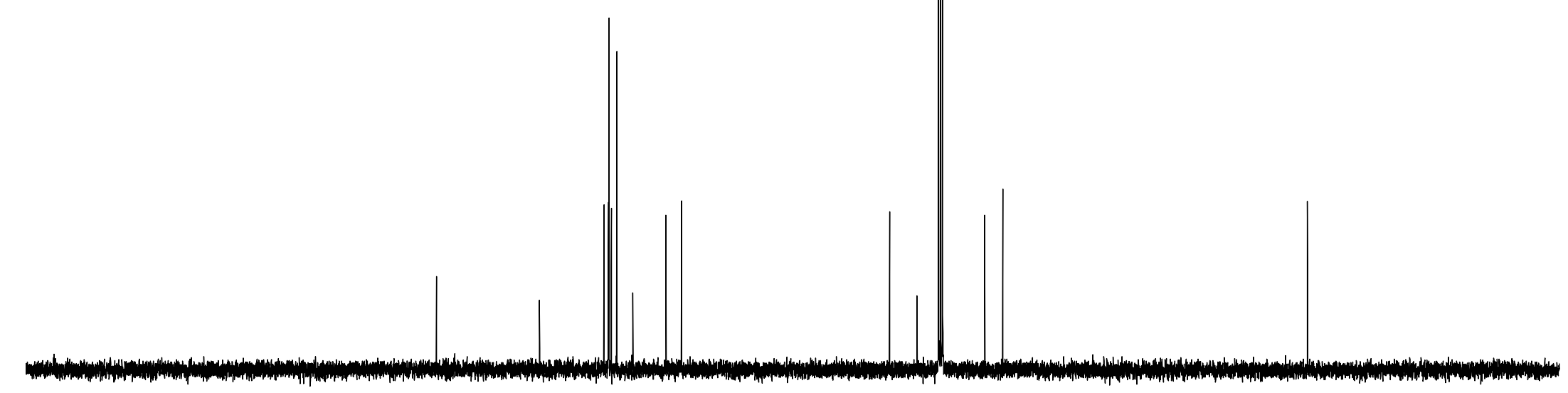

$200 \quad 190 \quad 180$

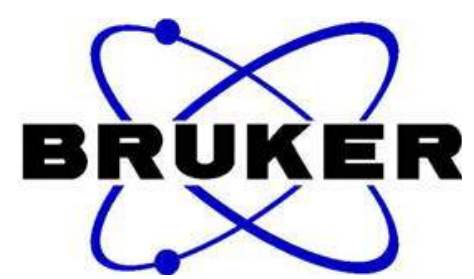

Current Data Parameters

NAME Izw3027D-C

PROCNO

1

F2 - Acquisition Parameters

Date_ 20150606

Time

20.47

INSTRUM spect

PROBHD $5 \mathrm{~mm}$ PABBO BB-

PULPROG $\quad$ zgpg30

TD

SOLVENT

6536

NS

$\begin{array}{lc}\text { DS } & 2 \\ \text { SWH } & 24038.461 \mathrm{~Hz}\end{array}$

FIDRES $\quad 0.366798 \mathrm{~Hz}$

$\mathrm{AQ} \quad 1.3631988 \mathrm{sec}$

RG 2050

DW 20.800 usec

DE $\quad 6.00$ usec

$\begin{array}{lc}\text { TE } & 300.0 \mathrm{~K} \\ \text { D1 } & 2.00000000 \mathrm{sec}\end{array}$

$\begin{array}{ll}\text { D1 } & 2.00000000 \mathrm{sec} \\ \text { d11 } & 0.03000000 \mathrm{sec}\end{array}$

DELTA $\quad \begin{aligned} & 0.89999998 \mathrm{sec} \\ & \text { Tec }\end{aligned}$

TDO

\begin{tabular}{|c|c|}
\hline $\begin{array}{l}\text { NUC1 } \\
\text { P1 } \\
\text { PL1 } \\
\text { SF01 }\end{array}$ & $\begin{array}{c}=\text { CHANNEL } \mathrm{f} 1==== \\
13 \mathrm{C} \\
9.25 \mathrm{usec} \\
-3.00 \mathrm{~dB} \\
100.6228298 \mathrm{MHz}\end{array}$ \\
\hline $\begin{array}{l}\text { ===== } \\
\text { CPDP } \\
\text { NUC2 } \\
\text { PCPD } \\
\text { PL12 } \\
\text { PL13 } \\
\text { PL2 } \\
\text { SFO2 }\end{array}$ & $\begin{array}{c}==\text { CHANNEL f2 ===== } \\
\text { w2 } \\
\text { waltz16 } \\
1 \mathrm{H} \\
80.00 \text { usec } \\
12.45 \mathrm{~dB} \\
18.00 \mathrm{~dB} \\
-1.00 \mathrm{~dB} \\
400.1316005 \mathrm{MHz}\end{array}$ \\
\hline $\begin{array}{l}\text { F2 - P } \\
\text { S1 } \\
\text { SF } \\
\text { WDW } \\
\text { SSB } \\
\text { LB } \\
\text { GB } \\
\text { PC }\end{array}$ & $\begin{array}{c}\text { cessing parameters } \\
32768 \\
100.6127725 \mathrm{MHz} \\
\mathrm{EM} \\
0 \\
1.00 \mathrm{~Hz} \\
0 \\
1.40\end{array}$ \\
\hline
\end{tabular}




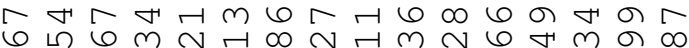

अ $m m m m \sim N \sim \sigma \infty \infty \infty 6$

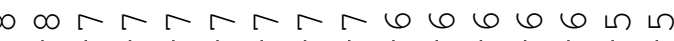

Y

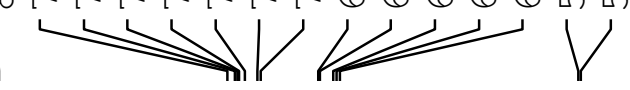

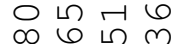

$\infty \infty \infty \infty$

$\dot{m} \dot{m} \dot{m} \dot{m}$

N/
강

각

$\gamma$<smiles>CC(C)OC(c1ccccc1)c1ccccc1O</smiles>

$2 r$

\section{en $(->)$}

Current Data Parameters

NAME

lzw2102B-H

PROCNO

F2 - Acquisition Parameter

Date__ 20150402

$\begin{array}{lr}\text { Time } & 16.39\end{array}$

INSTRUM spect

PROBHD $5 \mathrm{~mm}$ PABBO BB/

PULPROG

$\mathrm{mg} 30$
65536

TD

NS

NS

DSH

SWH

$\mathrm{AQ}$

RG

DW
DE
TE

DD 0

$====$

$\mathrm{SFO1}$

NUC

P1

$\mathrm{Zg} 30$
65536

7

$8012.820 \mathrm{~Hz}$

$4.0894465 \mathrm{sec}$ 70.97

62.400 usec 6.50 usec

$1.00000000 \mathrm{sec}$

CHANNET 11 $400.1324710 \mathrm{MHz}$ $1 \mathrm{H}$ 14.50 usec

F2 - Processing parameters

SI $\quad 65536$

SF $\quad 400.1300000 \mathrm{MHz}$

WDW

SSB

EM

$0.30 \mathrm{~Hz}$

1.00 

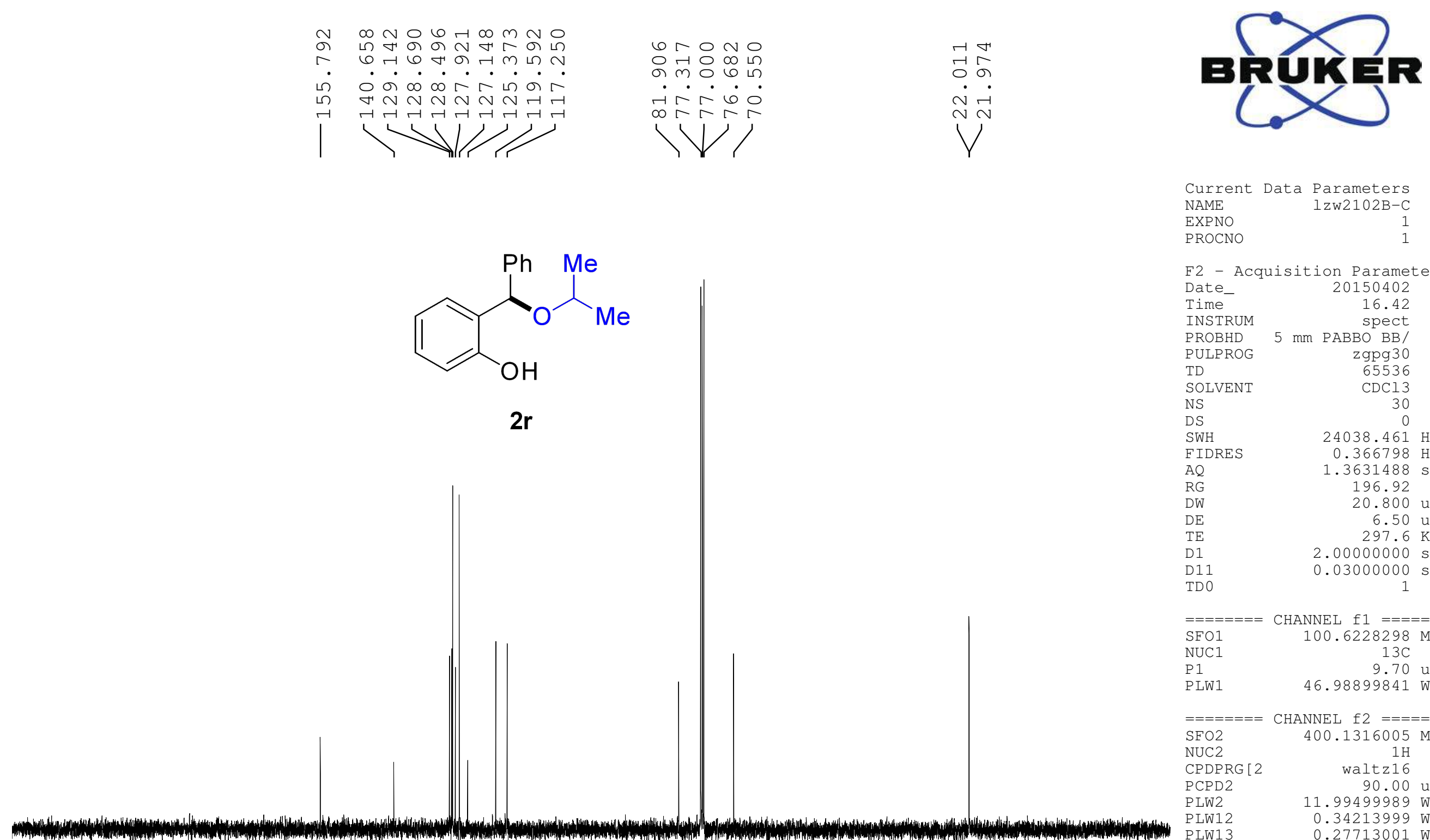

$\begin{array}{ll}\text { Current Data Parameters } \\ \text { NAME } & \text { lzw2102B-C }\end{array}$

PROCNO

1

F2 - Acquisition Parameters

Date_ 20150402

Time 16.42

spect

zgpg30
65536

SOLVENT CDCl3

NS

SWH

FIDRES

FIDR
$\mathrm{AQ}$

AQ

$\mathrm{DW}$

$\mathrm{DE}$

D1

D11

$======$

$\mathrm{SFO} 1$
$\mathrm{NUC1}$

$\mathrm{NUC}$

P1
PLW1

$24038.461 \mathrm{~Hz}$

$1.3631488 \mathrm{sec}$ 196.92

20.800 usec 6.50 use

$2.00000000 \mathrm{sec}$

$0.03000000 \mathrm{sec}$

CHANNET 61 = $=0$

$100.6228298 \mathrm{MHz}$

9.70

9.70 use

$=-2$

$\mathrm{SFO} 2$

NUC2

CPDPRG [2

PCPD2
PLW2

PLW13

HANNEL $12========$

$400.1316005 \mathrm{MHz}$

waltz16

1.990 .00 usec $0.34213999 \mathrm{~W}$

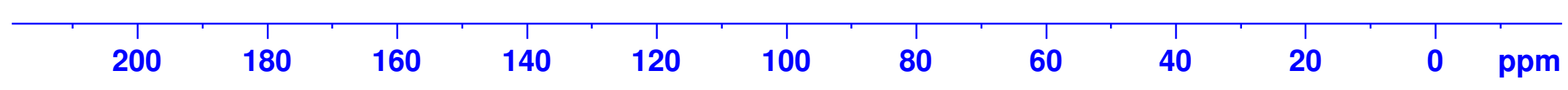

F2 - Processing parameters SI 32768

SF $\quad 100.6127741 \mathrm{MHz}$ 


\section{en $(>$}

Current Data Parameters NAME

PROCNO

lzw2189A-H

F2 - Acquisition Parameters<smiles>Oc1ccccc1C(OC1CCC1)c1ccccc1</smiles>

Date_

20150519

Time $\quad 16.14$

$5 \mathrm{mmect}$

PULPROG

$\mathrm{zg} 30$
65536

SOLVENT CDCl3

STV

DS

SWH

FIDRES

$2 s$

AQ

RG

DW

DE

TE

TD 0

$====$
$\mathrm{SFO1}$

$\mathrm{N} 1$

$\mathrm{CDCl}$
6

$8012.820 \mathrm{~Hz}$

$0.122266 \mathrm{~Hz}$

4.0894465
34.77 34.77

62.400 usec 6.50 usec

$1.00000000 \mathrm{sec}$
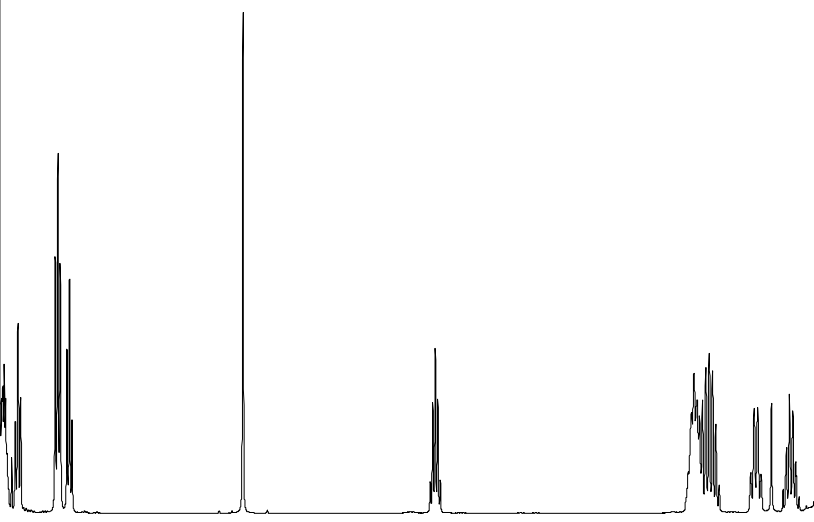

PLW

CHANNEL $\mathrm{fl}=======$ $400.1324710 \mathrm{MHz}$ 14.50 usec

Processing parameter

SI 65536

$\begin{array}{ll}\text { SF } & 400.1300088 \mathrm{MHz}\end{array}$

TSB

EM

$0.30 \mathrm{~Hz}$

1.00

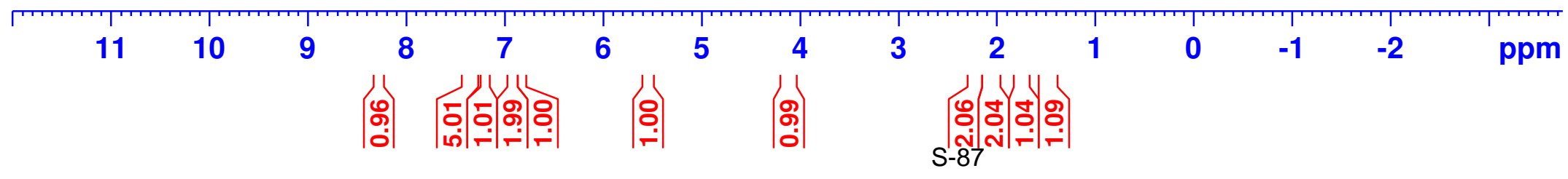



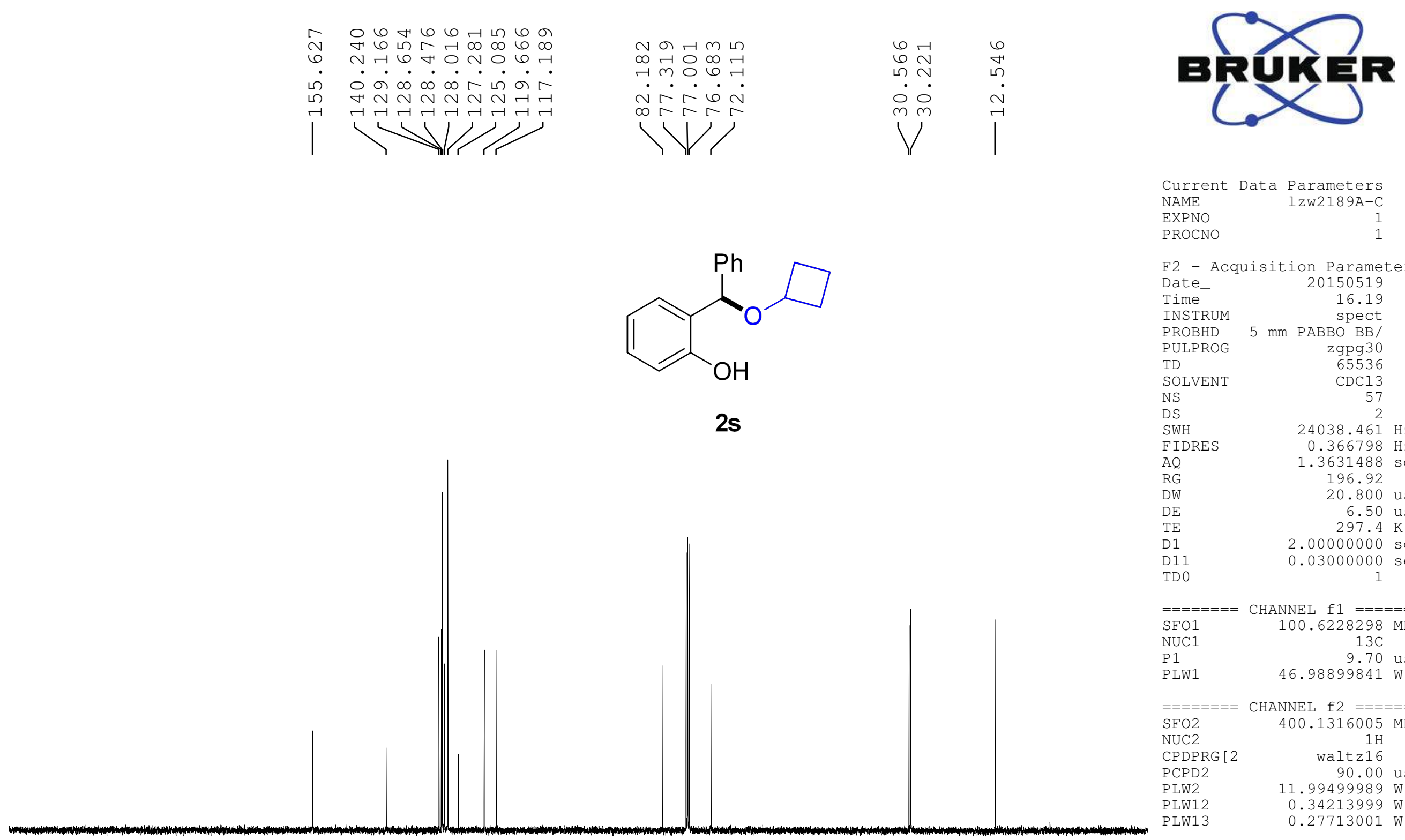

$\begin{array}{lr}\text { Current } & \text { Data } \\ \text { NAME } & \text { lzw2189A-C } \\ \text { EXPNO } & 1 \\ \text { PROCNO } & 1\end{array}$

F2 - Acquisition Parameters Date__ 20150519

$\begin{array}{lr}\text { Time } & 16.19\end{array}$

INSTRUM spect

zgpg30

$\begin{array}{ll}\text { TD } & 65536 \\ \text { CDCIENT } & 57\end{array}$

NS

SWH

FIDRES

FIDR
AQ
RG
DW

DW
DE
TE
D1

D1

D11
TD 0

$======$

$\mathrm{SFO1}$

P 1
PLW1

$24038.461 \mathrm{~Hz}$ $0.366798 \mathrm{~Hz}$ $1.3631488 \mathrm{sec}$ 196.92

20.800 usec 6.50 use

$2.00000000 \mathrm{~K}$ $0.03000000 \mathrm{sec}$

$=======$ CHANNEL $\mathrm{f} 2========$ FO2 $400.1316005 \mathrm{MHz}$

NUC2

CPDPRG [2

PCPD2

PLW2

PLW12
PLW13

CHANNEL $\mathrm{f} 1========$

$100.6228298 \mathrm{MHz}$

9.70 usec

waltz16

$11.99499989 \mathrm{~W}$

$0.34213999 \mathrm{~W}$
$0.27713001 \mathrm{~W}$

F2 - Processing parameters
SI

\begin{tabular}{rrrrrr|rrrrrr}
\hline 200 & 180 & 160 & 140 & 120 & 100 & 80 & 60 & 40 & 20 & 0 & $p p m$
\end{tabular}

SF $\quad 100.6127770 \mathrm{MHz}$

$\begin{array}{ll}\text { WDW } & \text { EM } \\ \text { SSB } & 0\end{array}$

$\begin{array}{lll}\mathrm{LB} & 1.00 \\ \mathrm{~GB} & 0 & \\ \mathrm{PC} & & 1.40\end{array}$ 


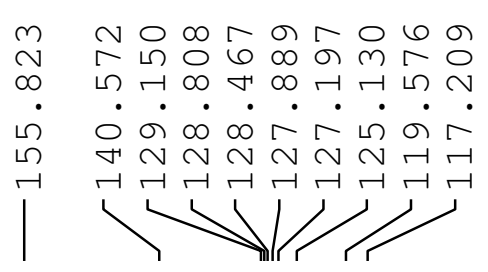

Ar

เा $\mathrm{r}$ ल 6

Nor $\circ \dot{r}$

$\backslash 1$

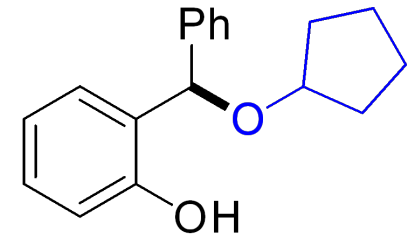

$2 t$

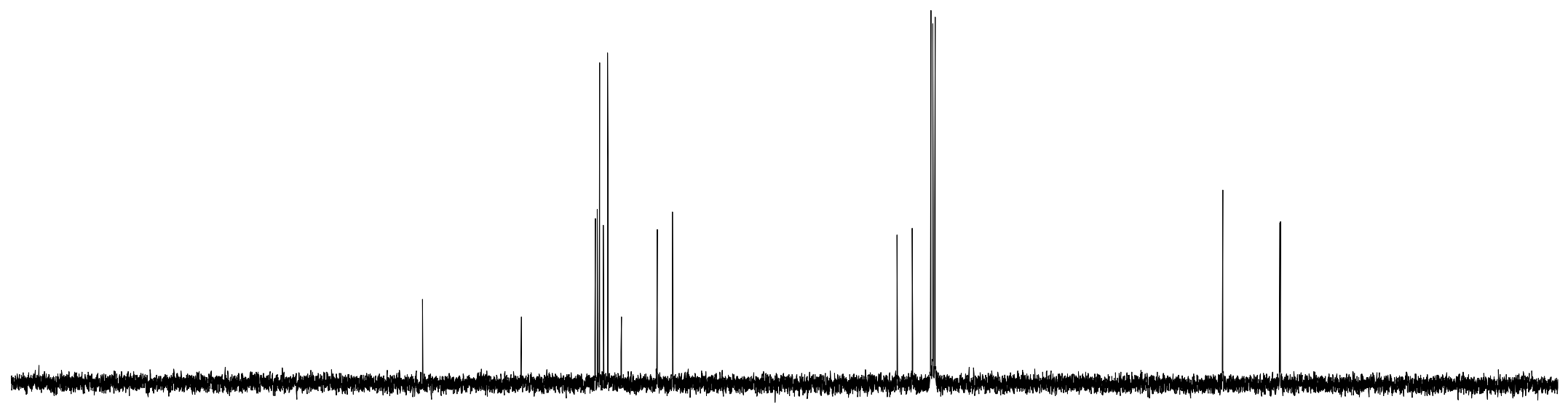

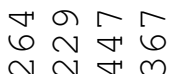

$\cdot \dot{\sim} \dot{m} \cdot$

$m \sim \sim$

VY
120

80

60

\section{BR $(1)$}

Current Data Parameters

NAME

PROCNO

1zw2102D-C

F2 - Acquisition Parameters

Date__ 20150402

Time $\quad 16.33$

spect

zgpg30

SOLVENT

NS

DS

$\begin{array}{lr}\text { SWH } & 24038.461 \mathrm{~Hz} \\ \text { FIDRES } & 0.366798 \mathrm{~Hz}\end{array}$

DW

$\mathrm{DE}$

TE

D1
D11

TD 0

$=====$

$\mathrm{SFO} 1$
$\mathrm{NUC1}$

$\mathrm{NUC}$

PLW1

$1.3631488 \mathrm{sec}$ 196.92

20.800 usec 6.50 use

$2.00000000 \mathrm{kec}$

$2.00000000 \mathrm{sec}$ $0.03000000 \mathrm{sec}$

CHANNEL $\mathrm{f1}========$ $100.6228298 \mathrm{MHz}$ $13 \mathrm{C}$

9.70 usec
$46.98899841 \mathrm{~W}$

$=======$ CHANNEL $\mathrm{f} 2========$ FO2 $\quad 400.1316005 \mathrm{MHz}$

CPDPRG [

PCPD 2

PLW12
PLW13

$1 \mathrm{H}$

waltz16 0.00 usec

$11.99499989 \mathrm{~W}$ $0.34213999 \mathrm{~W}$
$0.27713001 \mathrm{~W}$

SI - Processing parameters SF $\quad 32768$

$100.6127754 \mathrm{MHz}$

$\begin{array}{llll}40 & 20 & 0 & \text { ppm }\end{array}$

LB

$1.00 \mathrm{~Hz}$

1.40 
○ங

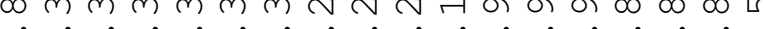

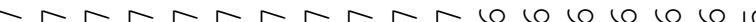

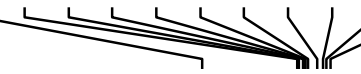

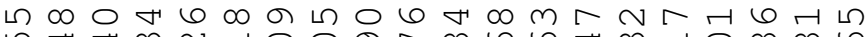
ก

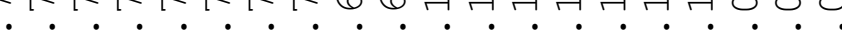
$m m m m m m m m m \sim \sim \sim \sim \sim \sim \sim \sim \sim$

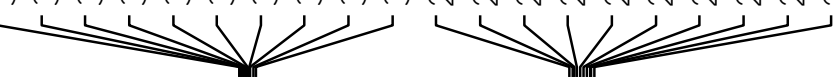
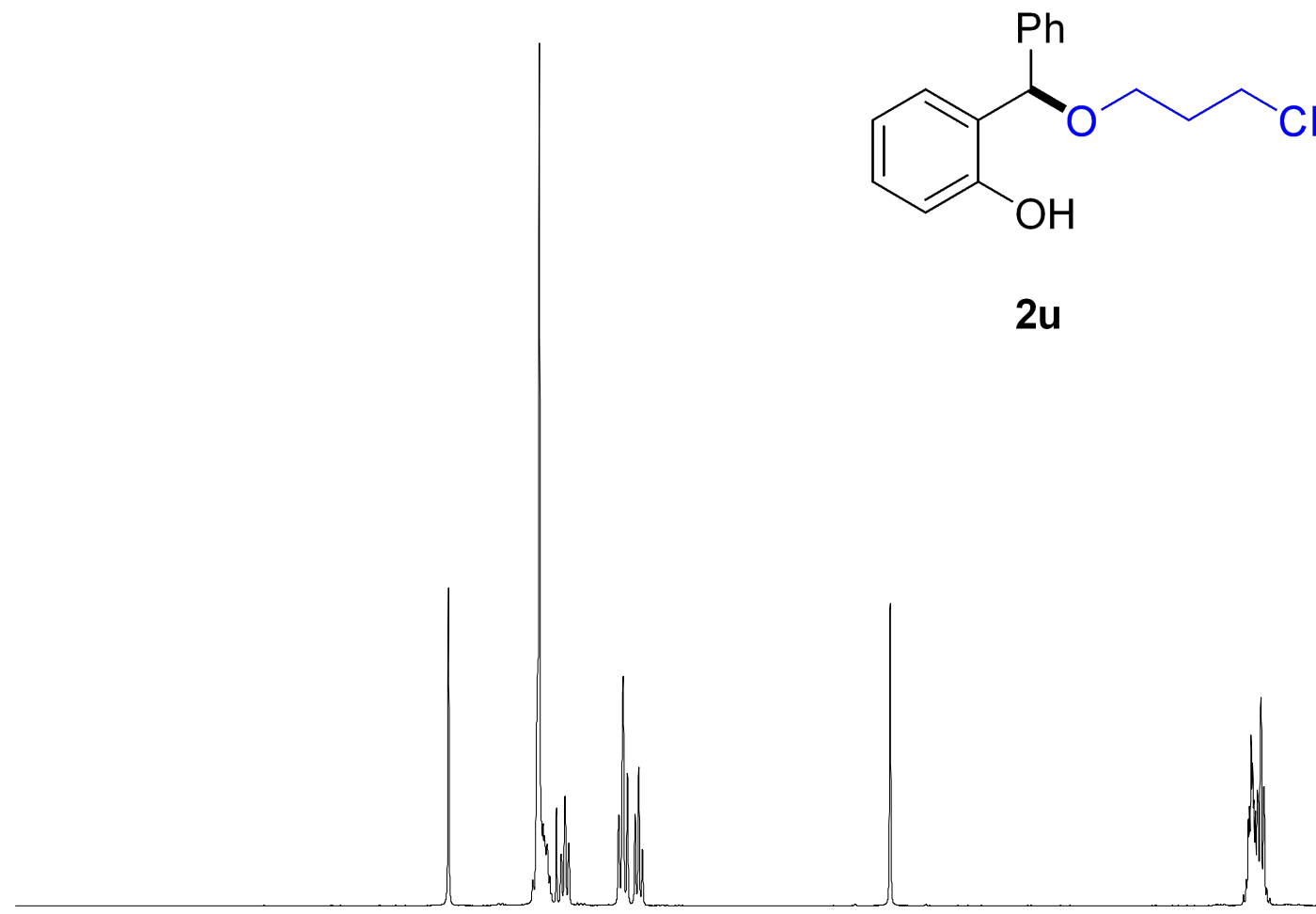

$2 \mathrm{u}$

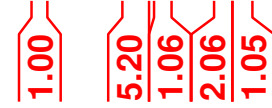

6

$\left|\begin{array}{l}0 \\ 0 \\ -1\end{array}\right|$

5
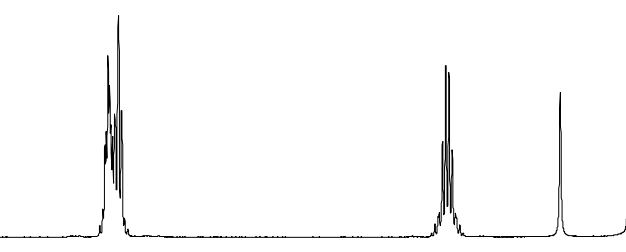

\section{Be $(1<)$}

$\begin{array}{ll}\text { Current Data Parameters } \\ \text { NAME } & \text { lzw3113A-H }\end{array}$

EXPNO

1
1

PROCNO

F2 - Acquisition Parameters

Date_ 20150730

Time $\quad 19.26$

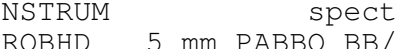

ROBHD

ZUL30

TD $\quad 65536$

OLVENT

NS

DS

FIDRES

$\mathrm{AQ}$

RG

DW

$\mathrm{DE}$

TE

TD 0

$===$

$\mathrm{SFO1}$

$\mathrm{NUC}$
$\mathrm{P} 1$

$\mathrm{CDC} 13$
5

$8012.820 \mathrm{~Hz}$

$0.122266 \mathrm{~Hz}$

$4.0894465 \mathrm{sec}$ 82.92

62.400 usec $\begin{array}{rl}6.50 & \text { use } \\ 295.9 & K\end{array}$

$1.00000000 \mathrm{sec}$

$$
1
$$

CHANNEL $\mathrm{f} 1========$ $400.1324710 \mathrm{MHz}$ 14.50 us

$11.99499989 \mathrm{~W}$

2 - Processing parameters

SI $\quad 65536$

SF $\quad 400.1300095 \mathrm{MHz}$

WDW

EM

$0.30 \mathrm{~Hz}$

1.00 


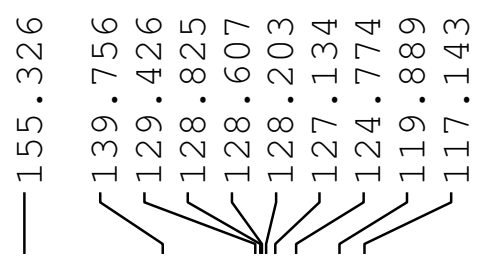

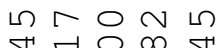

0 m० $0 \mathrm{~N}$

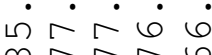

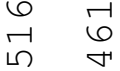

\section{BR $(1)$}

Current Data Parameters

NAME

lzw3113A-C

PROCNO

1

F2 - Acquisition Parameters

Date_ 20150730<smiles>Oc1ccccc1C(OCCCCl)c1ccccc1</smiles>

Time

PROBHD

PULPROG zgpg30

19.30
spect

$5 \mathrm{~mm} \mathrm{PABBO} \mathrm{BB/}$

SOLVENT

SOLV
NS
DS

NS
DS
SWH

SWH

$2 \mathbf{u}$

$\mathrm{AQ}$

RG

DW

TE

D 1
D 11

TD 0

$======$

$\mathrm{SFO1}$
NUC1

P 1
PLW1

zgpg 30
65536

$\mathrm{CDCl} 3$

$24038.461 \mathrm{~Hz}$

$1.3631488 \mathrm{sec}$

196.92

20.800 usec

6.50 usec

$2.00000000 \mathrm{~K}$

$0.03000000 \mathrm{sec}$

CHANNET 61 =

$100.6228298 \mathrm{MHz}$ $13 \mathrm{C}$ $=======$ CHANNEL $\mathrm{f} 2$ $=======$

NUC2

CPDPRG [2

PCPD2

PLW2

PLW12

1 .

\begin{tabular}{|c|c|c|c|c|c|c|c|c|c|c|c|}
\hline $\begin{array}{c}1 \\
200\end{array}$ & $\begin{array}{c}1 \\
180\end{array}$ & $\begin{array}{c}1 \\
160\end{array}$ & 140 & $\begin{array}{c}1 \\
120\end{array}$ & $\begin{array}{c}1 \\
100\end{array}$ & 80 & 60 & 40 & 20 & $\begin{array}{l}1 \\
0\end{array}$ & pom \\
\hline
\end{tabular}

2 - Processing parameters SI 32768

SF $\quad 100.6127734 \mathrm{MHz}$

180

SSB
LB
GC

0

$1.00 \mathrm{~Hz}$

1.40 


\section{Bg $(-1)$}

Current Data Parameters NAME

EXPNO

lzw3103C-H

PROCNO

F2 - Acquisition Parameters

Date_ 20150724

Time 21.59

$5 \mathrm{mmect}$

$\mathrm{zg} 30$
65536

TD $\quad 65536$

NS

DSH

FIDRES

FIDR
$\mathrm{AQ}$

AQ

2v

\section{$8012.820 \mathrm{~Hz}$} $0.122266 \mathrm{~Hz}$ $4.0894465 \mathrm{sec}$ 82.92

62.400 usec 6.50 usec $1.00000000 \mathrm{sec}$

CHANNEI $f 1$ $400.1324710 \mathrm{MHz}$ SI $\quad 65536$ SF $\quad 400.1300096 \mathrm{MHz}$ WDW

SSB EM

$0.30 \mathrm{~Hz}$

1.00 


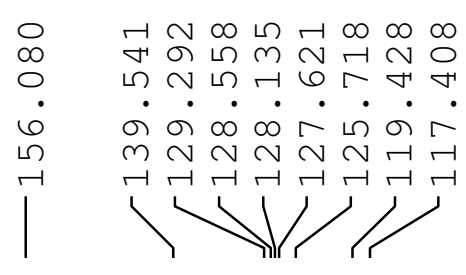

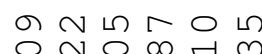

n molr

$\dot{\nabla} \therefore \dot{\sim} \dot{0} \dot{0}$

m ने

$|V|$

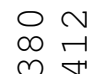
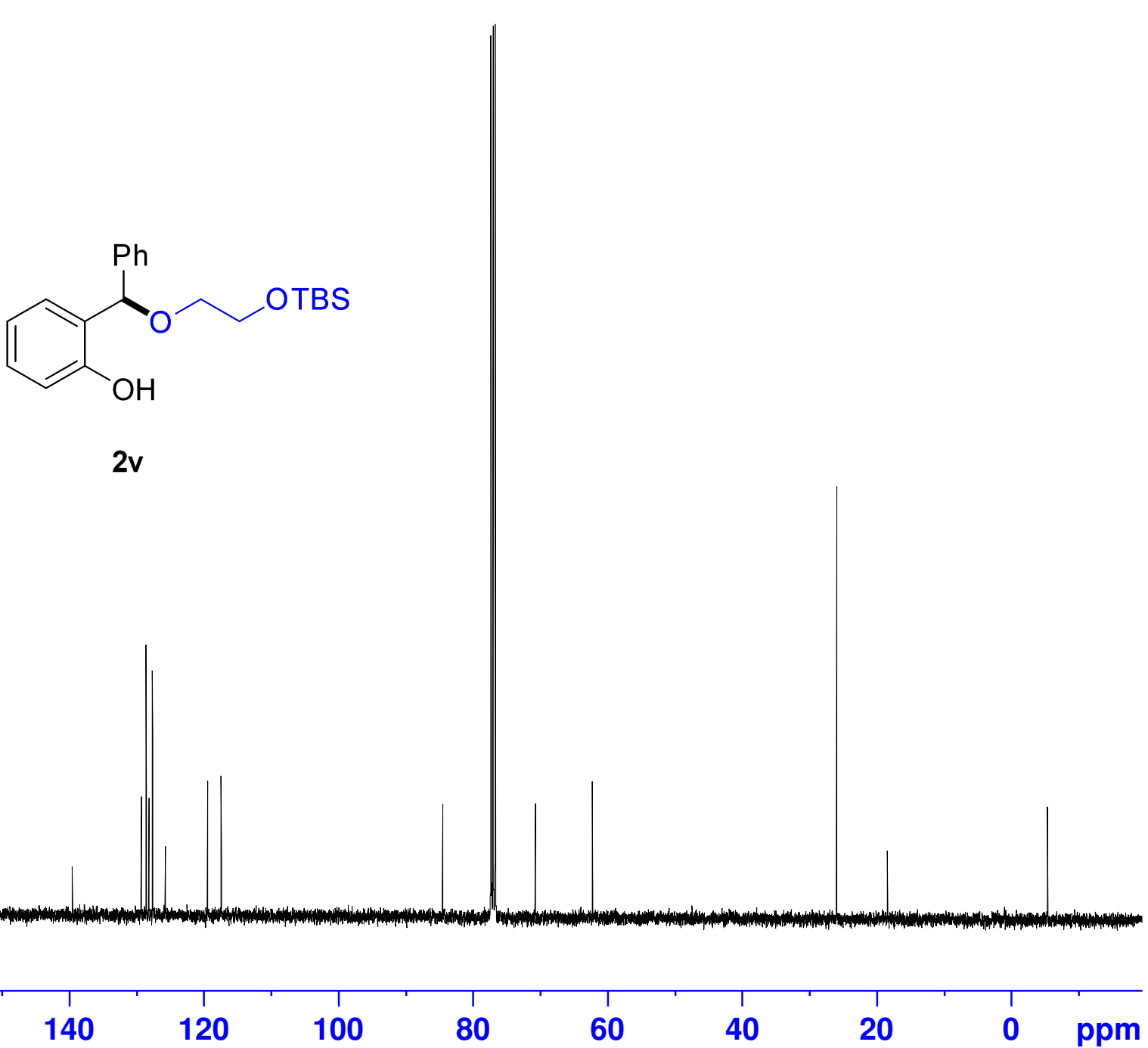

Current Data Parameters

NAME

lzw3103C-C

PROCNO

F2 - Acquisition Parameters

Date_ 20150724

$\begin{array}{lr}\text { Time } & 22.10\end{array}$

spect

zgpg30

$\begin{array}{lr}\text { IDOLVENT } & \text { CDCl3 } \\ \text { NS } & 178\end{array}$

DS

SWH

FIDRES

FIDR
AQ
RG

2v

DW

$\mathrm{TE}$

D 11

TD 0

$======$

$\mathrm{SFO1}$
$\mathrm{NUC1}$

P 1
PLW1

0
$24038.461 \mathrm{~Hz}$

$1.3631488 \mathrm{sec}$

196.92
20.800 usec

6.50 usec $2.00000000 \mathrm{sec}$
$0.03000000 \mathrm{sec}$

CHANNET 61 = $==2=$ $46.98899841 \mathrm{w}$ SEO2

$$
\text { NUC2 }
$$

CPDPRG [2

PCPD2

PLW2

PLW12

LW13

CHANNEL $f 2========$ $400.1316005 \mathrm{MHz}$ $1 \mathrm{H}$
waltzl6 $11.99499989 \mathrm{~W}$ $0.34213999 \mathrm{~W}$ $0.27713001 \mathrm{~W}$

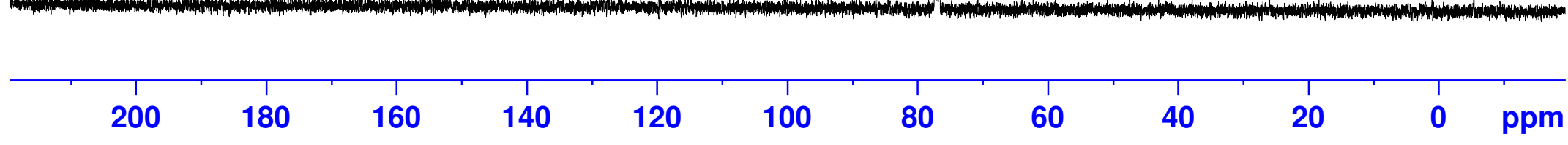

2 - Processing parameters I 32768 SF $\quad 100.6127708 \mathrm{MHz}$

160

140$$
100
$$ 


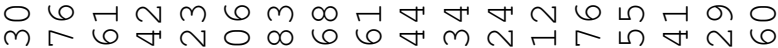

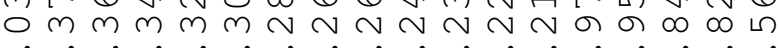

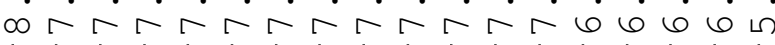
功

$\infty m \infty \sigma \sim+\infty \sim+m \infty \sim$

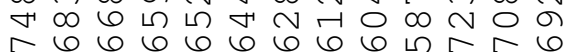
$\dot{m} \dot{m} \cdot \dot{m} \cdot \dot{m} \dot{m} \dot{\sim} \dot{\sim}$ m nn<smiles>O=S(=O)(CCOC(c1ccccc1)c1ccccc1O)c1ccccc1</smiles>

2w

\section{ex $(1)$}

Current Data Parameters NAME

EROCNO

lzw3117A-H

F2 - Acquisition Parameters

Date_ 20150730

Time $\quad 19.32$

$5 \mathrm{~mm}$ PABBO $\mathrm{BB} /$

PULPROG
zg30

SOLVENT CDCl3

NS

DS

SWH

DW

$\mathrm{DE}$

$\mathrm{TE}$

TD 0

$===$

$\mathrm{SFO}$

$\mathrm{NUC}$

P 1
PLW1

$4.0894465 \mathrm{sec}$ 82.92

62.400 usec 6.50 use $1.00000000 \mathrm{sec}$

CHANNEL $\mathrm{f} 1========$ $400.1324710 \mathrm{MHz}$ 14.50 sec $11.99499989 \mathrm{~W}$ SI 65536 SF $\quad 400.1300000 \mathrm{MH}$ SSB

$0.30 \mathrm{~Hz}$ 1.00

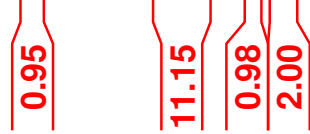

\section{5}

$\mid$

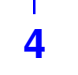

4

S†95|
3

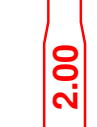




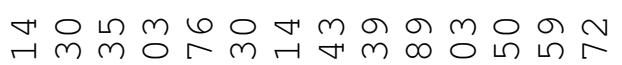

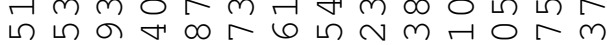
ம் $\dot{\sim} \dot{\sim} \infty \dot{\infty} \infty \dot{\infty} \dot{\infty} \dot{\sim} \dot{\sim} \dot{\sim} \dot{\sim}$

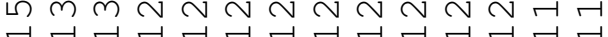

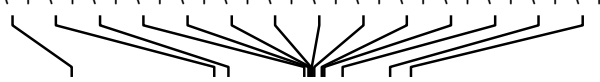

5 다의 $m$

$\infty \mathrm{m} \bigcirc 6 \mathrm{co}$

Ar

$|\vee|$

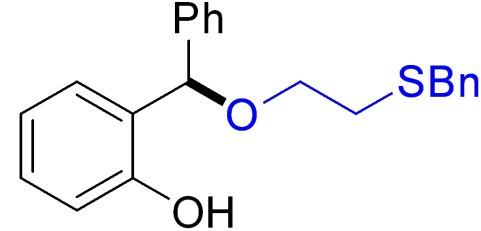

$2 w$

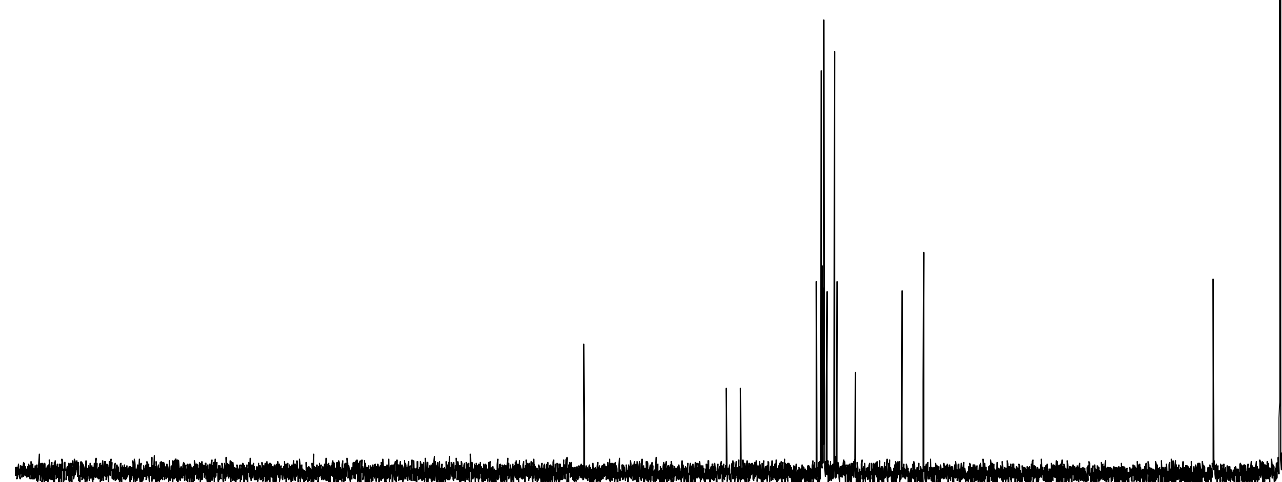

$\begin{array}{ll}m & 0 \\ m & 0\end{array}$

$\dot{6} \dot{0}$

1

\section{BR $(1)$}

Current Data Parameters NAME

EXPNO

lzw3117A-C

PROCNO

F2 - Acquisition Parameters

Date__ 20150730

Time $\quad 19.38$

spect

PULPROG

zgpg30

$\begin{array}{ll}\text { TD } & 65536 \\ \text { SOLVENT } & \text { CDCl3 }\end{array}$

DS

DWH

FIDRES

FIDR
AQ

AQ

DW

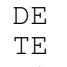

$\mathrm{TE}$
$\mathrm{D} 1$

D1 1

TD 0

$======$

$\mathrm{SFO1}$

$\mathrm{NUC1}$
$\mathrm{P} 1$

P1
PLW1

$=======$

$\mathrm{SFO} 2$

NUC2

CPDPRG [2

PCPD2

PLW2

PLW12

LW13

0.34213999

$0.27713001 \mathrm{~W}$

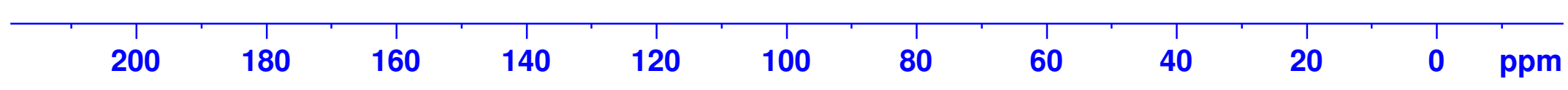

- Processing parameters SF

(2)




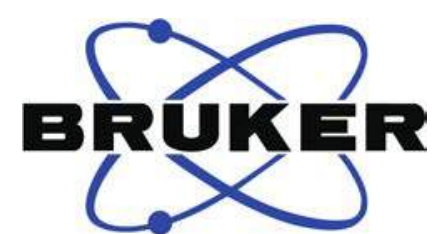

Current Data Parameters NAME

EXPNO

I ZW3117B-H

PROCNO

2 - Acquisition Parameters

Date_ 20150730

Time 22.18

$5 \mathrm{~mm}$ spect

\begin{tabular}{rr} 
PULPROG & zg30 \\
\hline
\end{tabular}

SOLVENT CDCl3

DS

SWH

FIDRES

$\mathrm{AQ}$

$2 x$

RG

$\mathrm{DW}$

$\mathrm{DE}$

$\mathrm{TE}$
$\mathrm{D} 1$

TD 0

$===$

$\mathrm{SFO}$

$\mathrm{NUC}$
$\mathrm{P} 1$

P 1
PLW1

0
$8012.820 \mathrm{~Hz}$

$0.122266 \mathrm{~Hz}$

82.92

62.400 usec 6.50 usec

$1.00000000 \mathrm{sec}$ 1

CHANNEL $\mathrm{fl}=======$ $400.1324710 \mathrm{MHz}$ $1 \mathrm{H}$ 4.50 usec 11.99499989 W SI 65536 SF $\quad 400.1300094 \mathrm{MHz}$ WDW

SSB

$0.30 \mathrm{~Hz}$

1.00
.

更

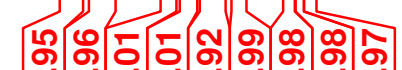

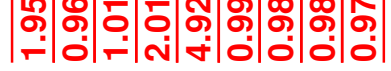

6 $\left|\begin{array}{l}\text { 임 } \\ 0\end{array}\right|$
5

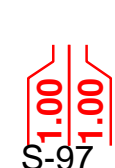

4 3 $\left|\begin{array}{l}\text { in } \\ \text { Ni }\end{array}\right|$
2

1 ppm 


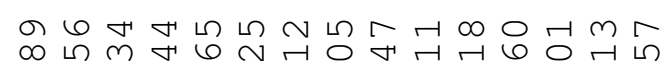

$\infty$ 네 $m$ न

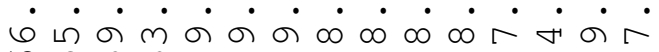

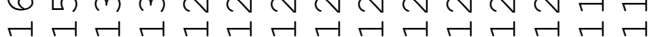

1

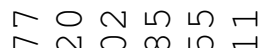

a m $06 \forall 6$

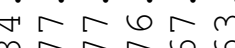

$|V| \mid$

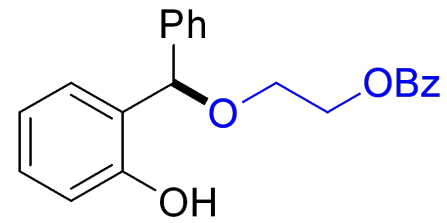

$2 x$

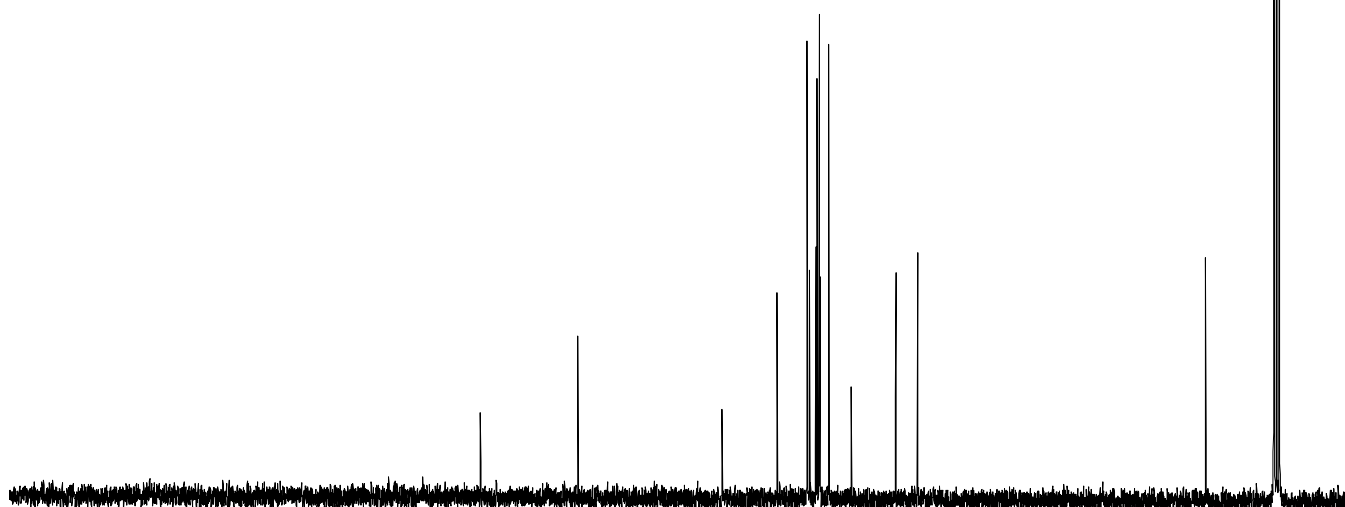

100
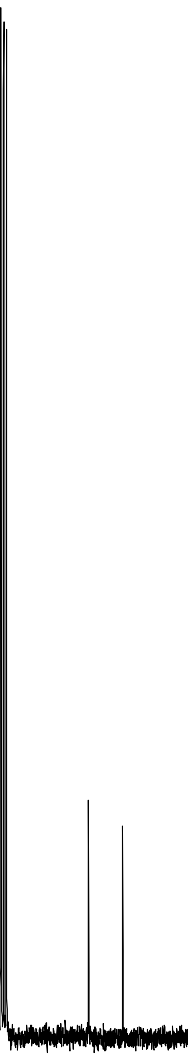

\section{0}

60

60

40 


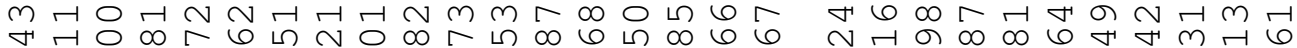
N F

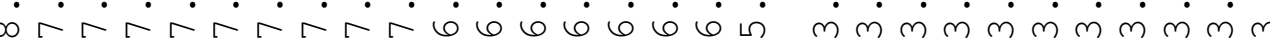
$\longrightarrow$
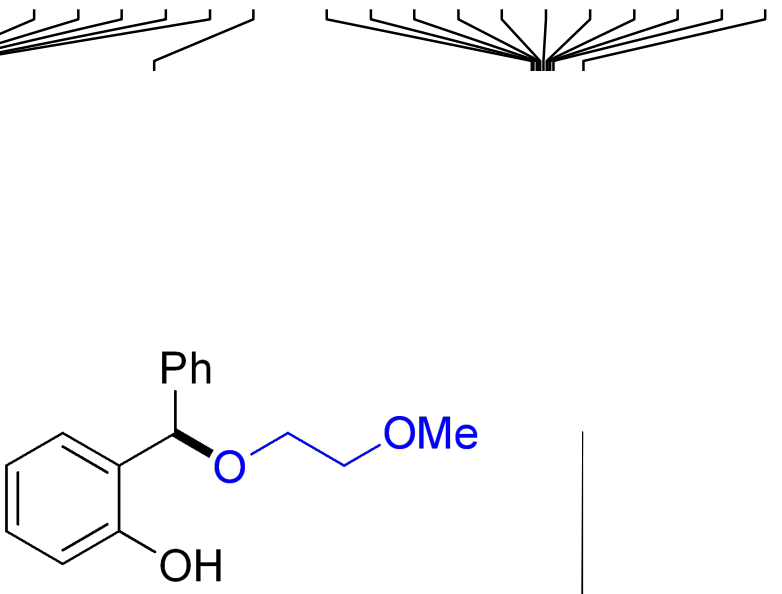

$2 y$

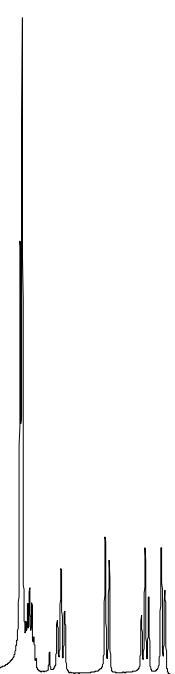

7

|
5

$\mid \begin{aligned} & \text { 운 } \\ & \mid\end{aligned}$

\section{en $(1)$}

Current Data Parameters

$$
\text { NAME }
$$

lzw3132B-H

PROCNO

F2 - Acquisition Parameters

Date_ 20150810

$\begin{array}{lr}\text { Time } & 23.55\end{array}$

INSTRUM spect

$\mathrm{zg} 30$
$\mathrm{gULPROG}$

TD 65536

OLVENT

NS

DS

FIDRES

$8012.820 \mathrm{~Hz}$
$\quad 0.122266 \mathrm{~Hz}$

RG

DW

$\mathrm{DE}$

TE

TDO

$======$

$\mathrm{SFO1}$

$\mathrm{NUC} 1$
$\mathrm{P} 1$

$4.0894465 \mathrm{sec}$
39.46

39.46
62.400 usec

6.50 usec

$298.2 \mathrm{~K}$

$1.00000000 \mathrm{sec}$

CHANNEL $\mathrm{f} 1========$

$400.1324710 \mathrm{MHz}$

14.50

$\begin{aligned} & 14.50 \text { usec } \\ & 11.99499989 \mathrm{~W}\end{aligned}$

F2 - Processing parameters

SI 65536

SF $\quad 400.1300089 \mathrm{MHz}$

WDW EM

$0.30 \mathrm{~Hz}$

GB
PC

1.00 

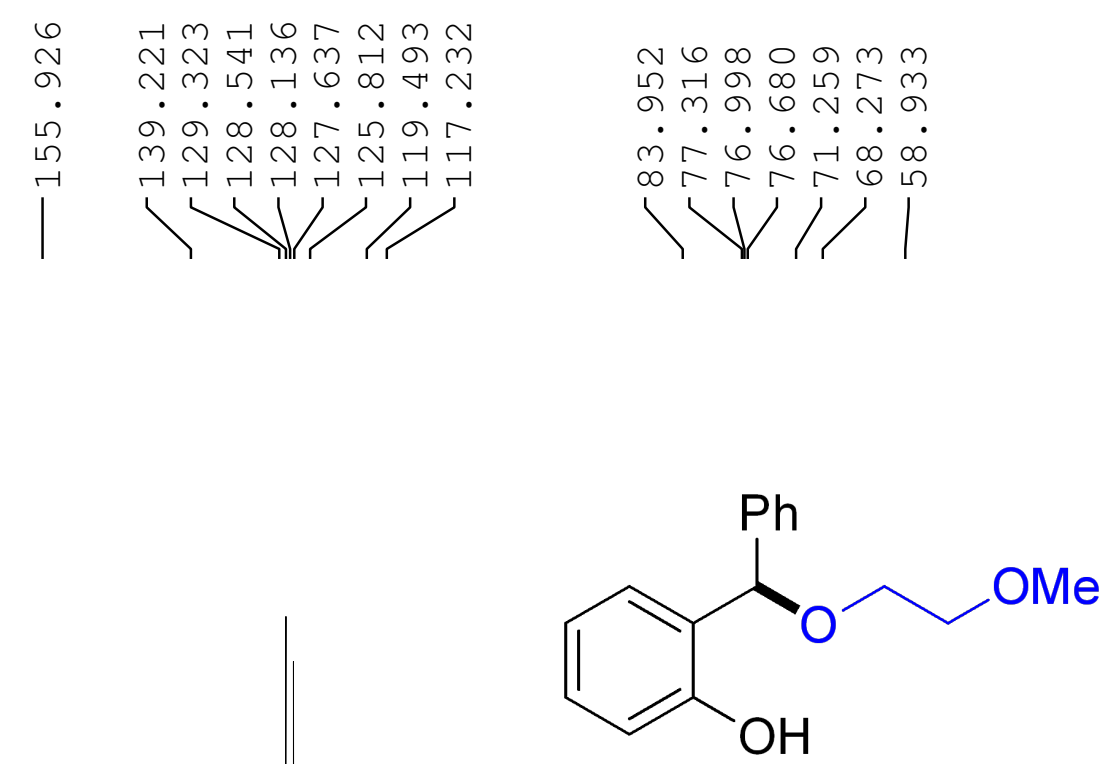

$2 y$

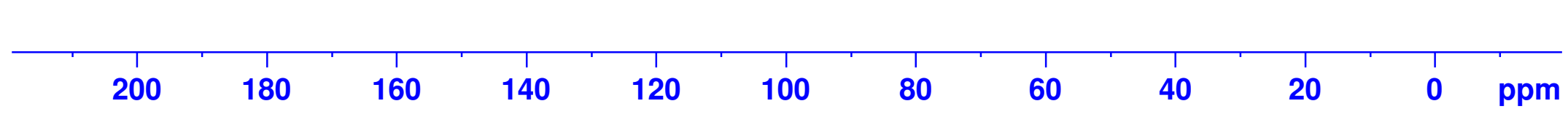

S-100

\section{BR $(1)$}

Current Data Parameters NAME

EXPNO
PROCNO

lzw3132B-C

F2 - Acquisition Parameters

Date_ 20150810

Time 23.58

Spect

PULPROG 5

zgpg30

$\begin{array}{ll}\text { TD } & 65536 \\ \text { NOLVENT } & \text { CDC13 }\end{array}$

DS

SWH

FIDRES

FIDR
$\mathrm{AQ}$

AQ
RG
$D W$

$\mathrm{DE}$

$\mathrm{TE}$
$\mathrm{D} 1$

D1 1

TD 0

$======$

$\mathrm{SFO1}$
$\mathrm{NUC1}$

$\mathrm{NUC}$

P 1
PLW1

$24038.461 \mathrm{~Hz}$

$\begin{array}{rl}1.3631488 & \mathrm{sec}\end{array}$

196.92

20.800 usec

6.50 usec

$299.1 \mathrm{~K}$
$.00000000 \mathrm{sec}$

$2.00000000 \mathrm{sec}$ $0.03000000 \mathrm{sec}$

CHANNEL $\mathrm{fl}========$ $100.6228298 \mathrm{MHz}$ $13 \mathrm{C}$

9.70 usec
$46.98899841 \mathrm{~W}$

$========$ CHANNEL $\mathrm{f} 2$ $========$ $\mathrm{SFO} 2$

NUC2

CPDPRG [2

PCPD2

PLW2

PLW12

PLW13

$400.1316005 \mathrm{MHz}$

$1 \mathrm{H}$
.

waltz16

90.00 usec
$11.99499989 \mathrm{~W}$

$0.34213999 \mathrm{~W}$

$0.27713001 \mathrm{~W}$

F2 - Processing parameters

SI 32768

SF $\quad 100.6127804 \mathrm{MHz}$ 


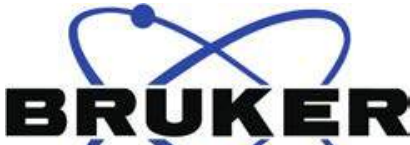 $(1)$}

Current Data Parameters NAME

PROCNO

lzw3119-H

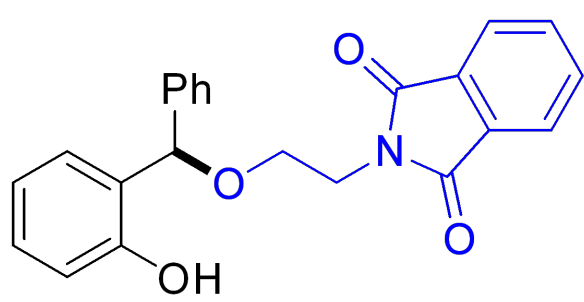

F2 - Acquisition Parameters

Date__ 20150731

22.02

INSTRUM

PROBHD

PULPROG

$5 \mathrm{~mm} \mathrm{PABBO} \mathrm{BB} /$

SOLVENT

NS

DS

SWH

IDR

$\mathrm{AQ}$

RG

DW

$\mathrm{DE}$

D 1
TD 0

$====$

$\mathrm{SFO1}$

NUC1

P1
PLW1

$z 930$
65536

5
5

\section{$8012.820 \mathrm{~Hz}$}

$0.122266 \mathrm{~Hz}$

$4.0894465 \mathrm{sec}$

70.97

62.400 usec

6.50 usec

1.000

CHANNEL f $1========$

$400.1324710 \mathrm{MHz}$

$1 \mathrm{H}$

14.50 usec

$11.99499989 \mathrm{~W}$

F2 - Processing parameters

SI $\quad 65536$

SF $\quad 400.1300000 \mathrm{MHz}$

SSB

SSB

EM

$0.30 \mathrm{~Hz}$

1.00

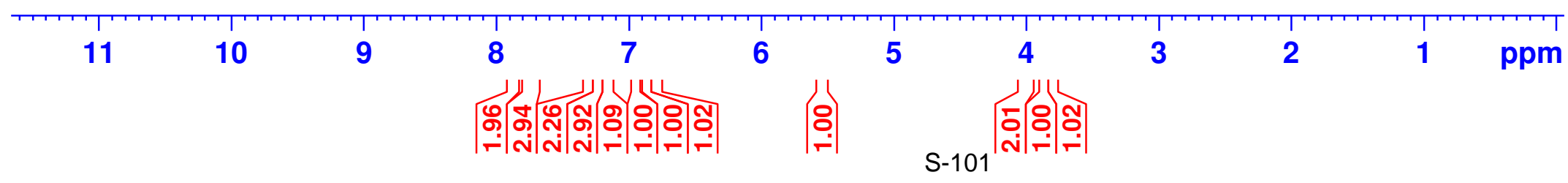


<smiles>O=C1c2ccccc2C(=O)N1CCOC(c1ccccc1)c1ccccc1O</smiles>

$2 z$
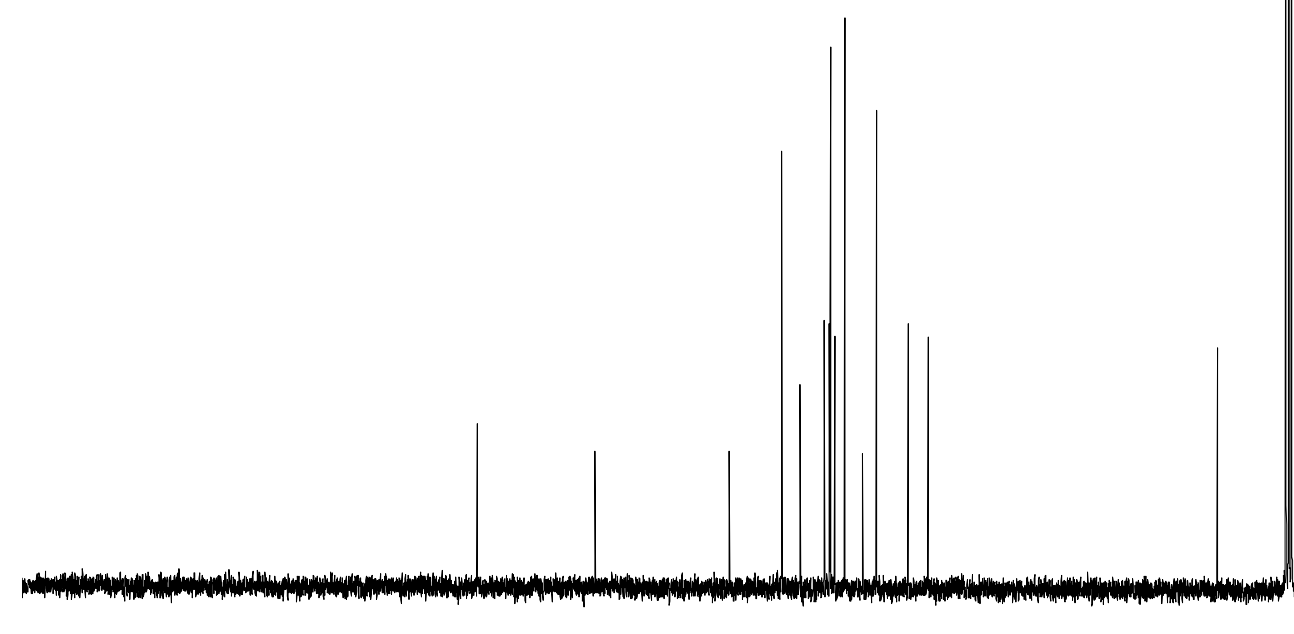

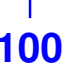

80
120
100

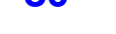

60

40

40




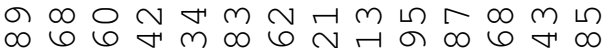

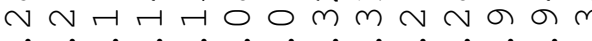

-rarrabib

$\rightarrow$ $\begin{array}{lllll}4 & r & 0 & 0 \\ 2 & 0 & 0\end{array}$ नન $m m m \sim$ M/1

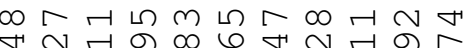
स म

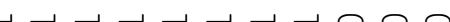

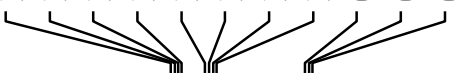

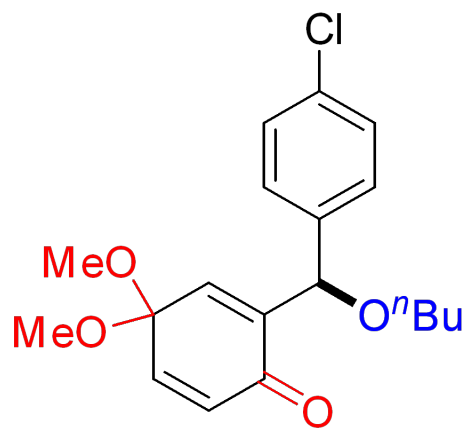

3
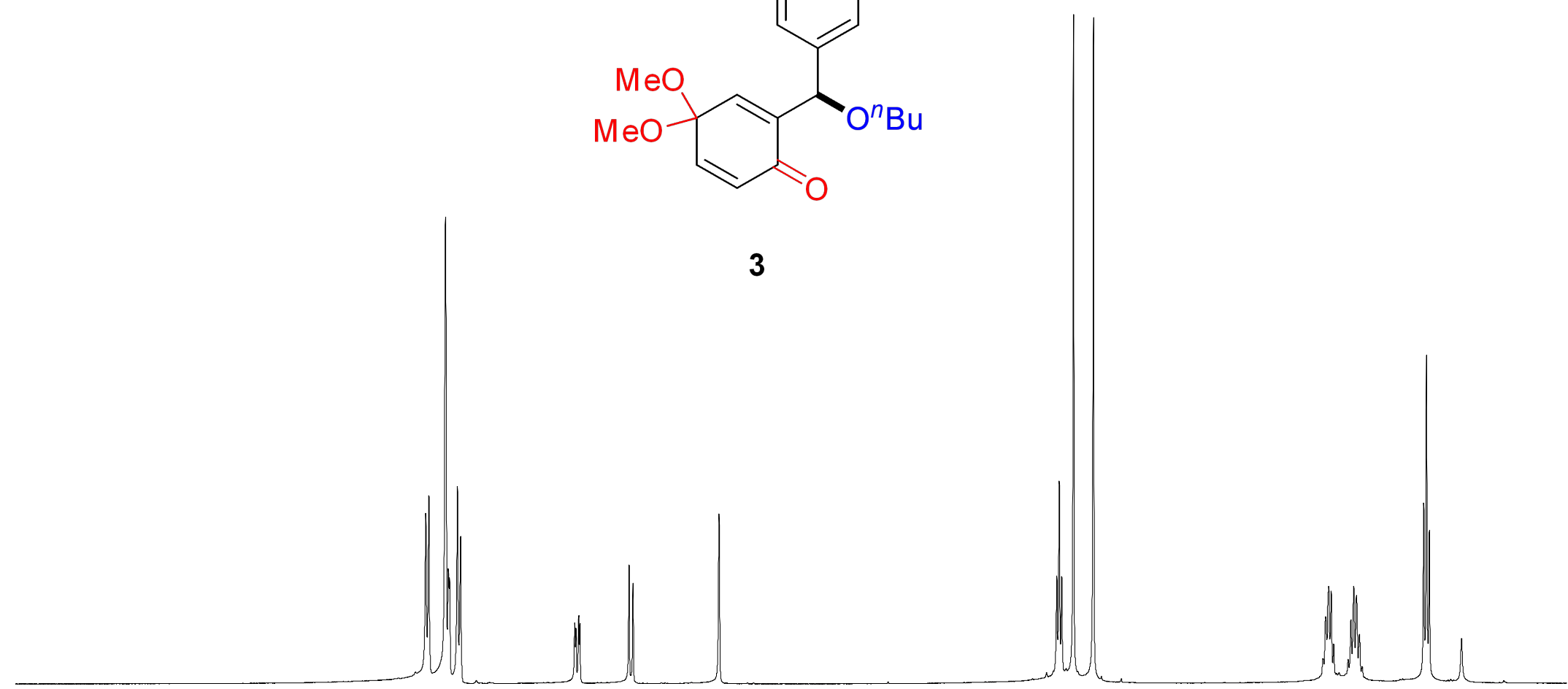

\section{eporer $(1)$}

Current Data Parameters NAME

PROCNO

lzw3079-H

F2 - Acquisition Parameters

Date__ 20150709

Time 14.42

spect

SOLVENT

NS

DS

AQ

RG

$\mathrm{DE}$

TE

TD 0

$===$

$\mathrm{SFO1}$

$\mathrm{NUC1}$
$\mathrm{P} 1$

$\mathrm{mm}$ PABBO $\mathrm{BB} /$
$\mathrm{zg} 30$

C6D 6

\section{$8012.820 \mathrm{~Hz}$}

$4.0894465 \mathrm{sec}$ 62.93

62.400 usec 6.50 usec

$1.00000000 \mathrm{sec}$

CHANNET $11=2=0$ $400.1324710 \mathrm{MHz}$

- Processing parameter

SI 65536

$\begin{array}{ll}\text { SF } & 400.1299961 \mathrm{MHz}\end{array}$

SSB

EM

$0.30 \mathrm{~Hz}$

1.00

$\begin{array}{llllllllllllllllllll}9.5 & 9.0 & 8.5 & 8.0 & 7.5 & 7.0 & 6.5 & 6.0 & 5.5 & 5.0 & 4.5 & 4.0 & 3.5 & 3.0 & 2.5 & 2.0 & 1.5 & 1.0 & \mathrm{ppm}\end{array}$

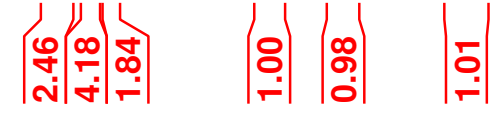




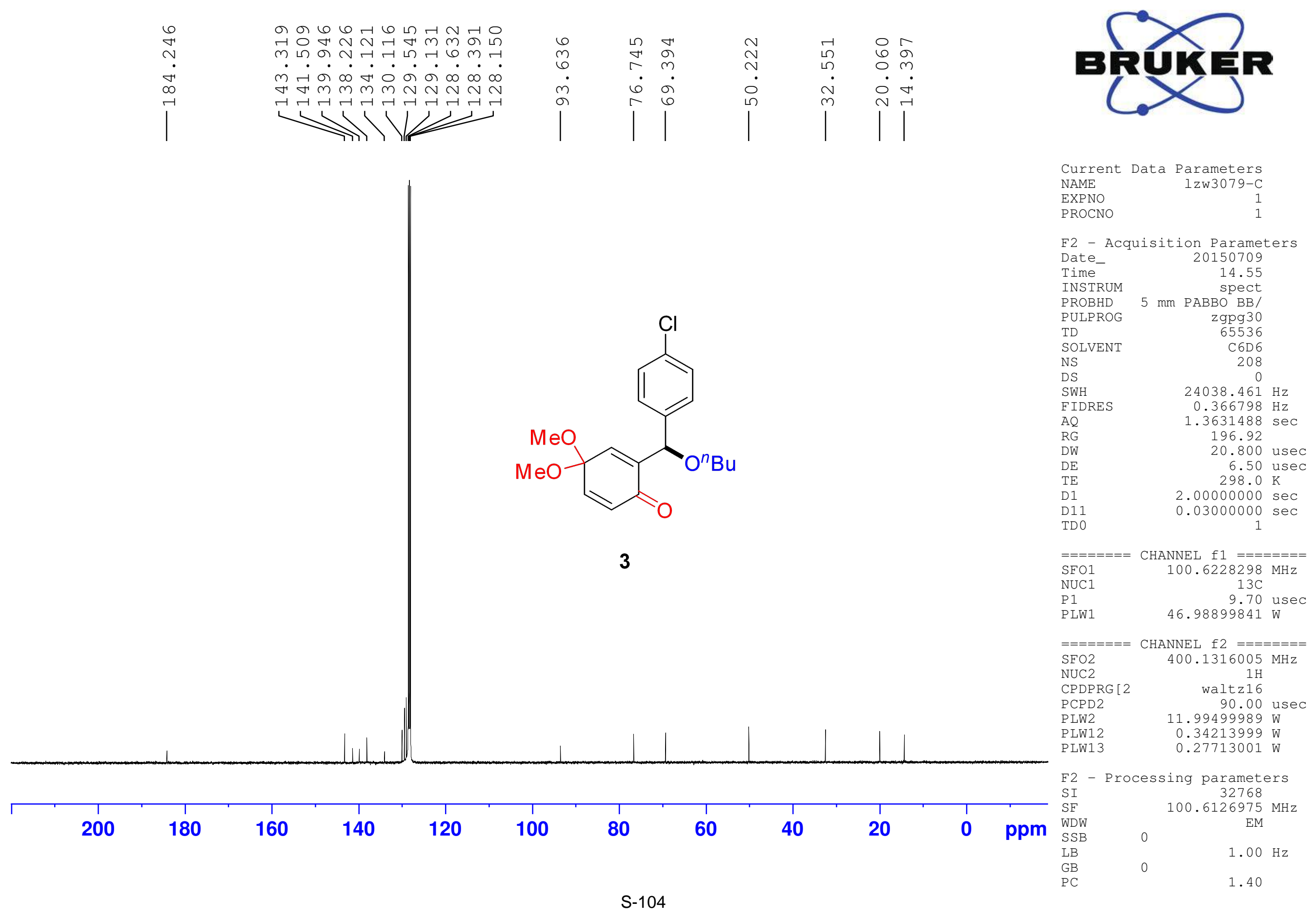


$\infty$ の

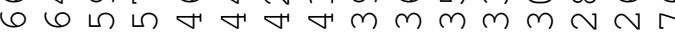

rarararararar

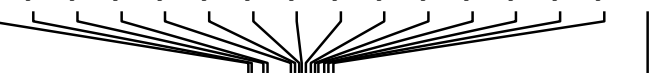

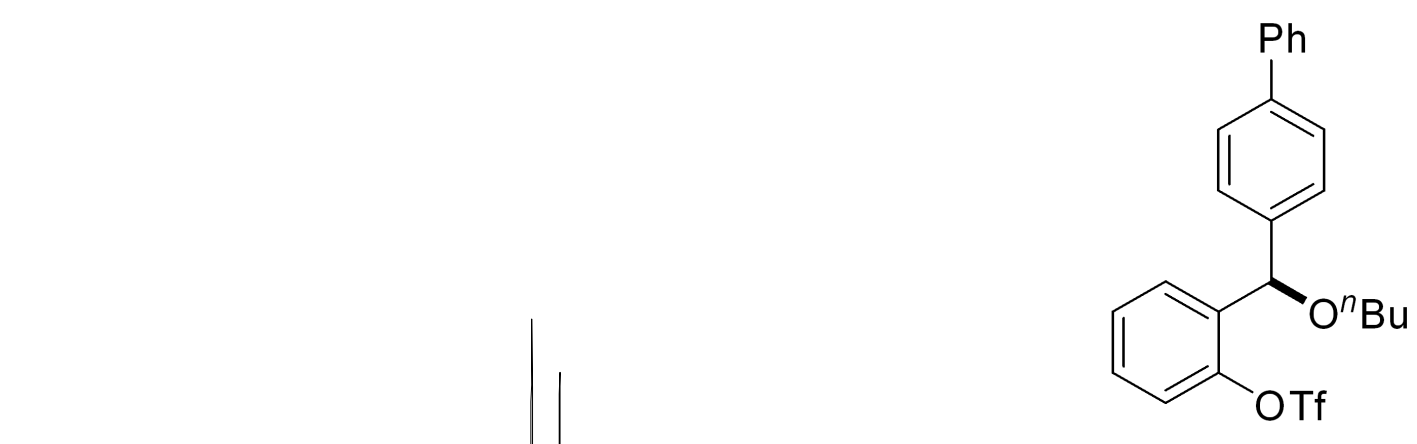

$2 e^{\prime}$

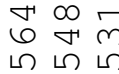

$\dot{m} \dot{m}$

$1 /$

H
न

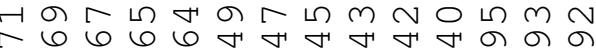

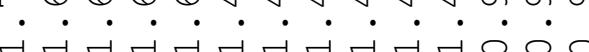

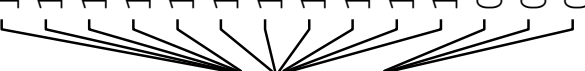

Current Data Parameters NAME

PROCNO

F2 - Acquisition Parameters

Date_

Time

PROBHD

PULPROG

TD

SOLVENT

NS

DS

FIDRES

AQ

RG

DW

$\mathrm{DE}$

D1

TD 0

$===$
$\mathrm{SFO} 1$

$\mathrm{SFO} 1$
$\mathrm{NUC1}$

P1

PLW1

F2 -
SI
SF

$\mathrm{SI}$
$\mathrm{SF}$
WDW

WDW
SSB

$\mathrm{LB}$
$\mathrm{GB}$

GB
PC

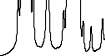

SUXER

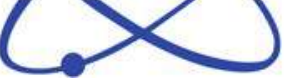

lzw3088-

1

20150716

$5 \mathrm{~mm}$ РABBO BB/

$\mathrm{zg} 30$
65536

$\mathrm{CDCl} 3$

$8012.820 \mathrm{~Hz}$

$8012.820 \mathrm{~Hz}$

$0.122266 \mathrm{~Hz}$

$$
54.81
$$

62.400 usec 6.50 usec

$1.00000000 \mathrm{sec}$

$$
1
$$

CHANNEL $f 1=$ $400.1324710 \mathrm{MHz}$ $1 \mathrm{H}$ 14.50 usec $11.99499989 \mathrm{~W}$

Processing parameter EM

$0.30 \mathrm{~Hz}$

1.00 $400.1300083 \mathrm{MHz}$ 


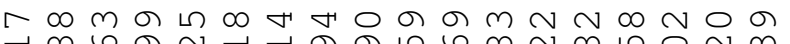

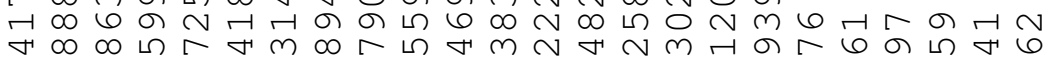

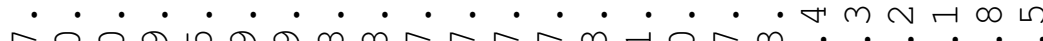

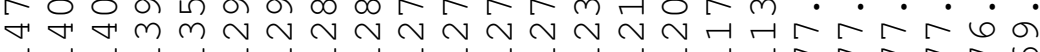

(N)
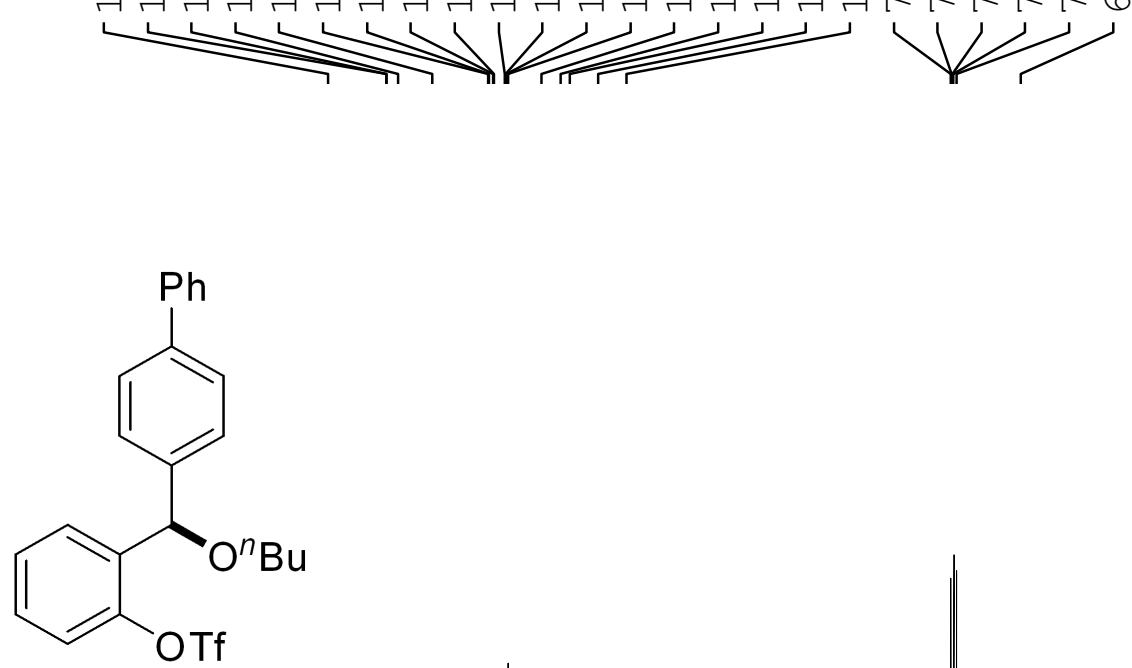

$2 e^{\prime}$

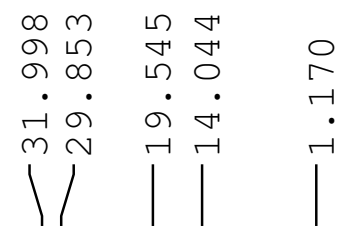

NAME

EXPNO

F2 - Acquisition Parameters Date_ 20150716

20.26

Time

PROBHD

PULPROG

SOLVENT

NS

DS

SWH

$\mathrm{AQ}$

RG

DW

TE

D1
D11

D11

$======$

$\mathrm{SFO} 1$

NUC

P 1
PLW1

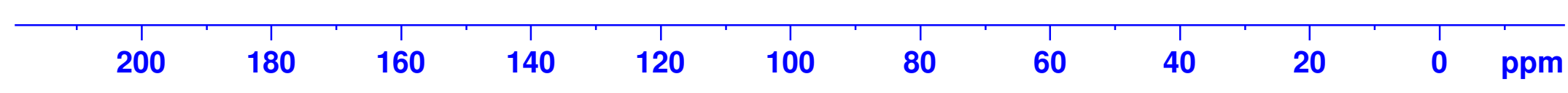

S-106
$\mathrm{SFO} 2$

NUC2

CPDPRG

PCPD2

PLW2

PLW12

PLW13

$0.27713001 \mathrm{~W}$

F2 - Processing parameters $\begin{array}{lr}\text { SI } & 32768 \\ \text { SF } & 100.6127568\end{array}$

$100.6127568 \mathrm{MHz}$ $13 \mathrm{C}$

NNEL $f 2=======$ $.1316005 \mathrm{MHz}$ waltz16 00 usec $1.99499989 \mathrm{~W}$ $0.34213999 \mathrm{~W}$ LB $\mathrm{PC}$

1.40 
ののமス ம

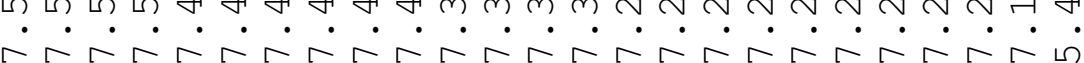

$\longrightarrow$
다 ॠ

$m m m$

$m \dot{m}$

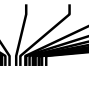

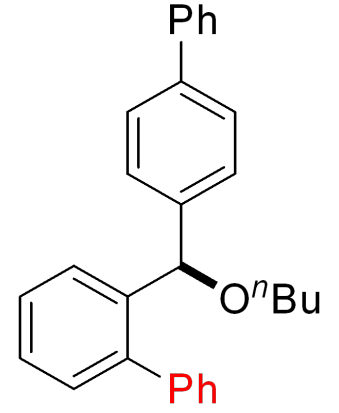

4

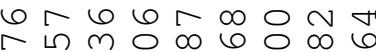

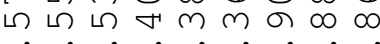

-

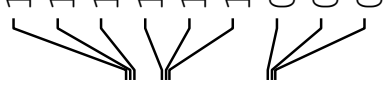

\section{se $(1>$}

Current Data Parameters NAME

PROCNO

lzw3124-H

2 - Acquisition Parameters

Date__ 20150801

Time $\quad 21.27$

$5 \mathrm{~mm}$ spect

PULPROG

zgB 30
65536

$\begin{array}{ll}\text { TD } & 65536 \\ \text { SOLVENT } & \text { CDCl3 }\end{array}$

TS

DS

SWH

FIDRES

$\mathrm{AQ}$

RG

DW

$\mathrm{DE}$

D1

TD 0

$===$

$\mathrm{SFO1}$

$\mathrm{NUC}$

PLW1

$8012.820 \mathrm{~Hz}$

$8012.820 \mathrm{~Hz}$

$4.0894465 \mathrm{sec}$

187.77

62.400 usec 6.50 usec

$1.00000000 \mathrm{sec}$

CHANNET f1 $=5=2=$ $400.1324710 \mathrm{MHz}$ 14.50 usec $11.99499989 \mathrm{~W}$

Processing parameters SI $\quad 65536$ SF $\quad 400.1300095 \mathrm{MHz}$

EM

$0.30 \mathrm{~Hz}$

1.00

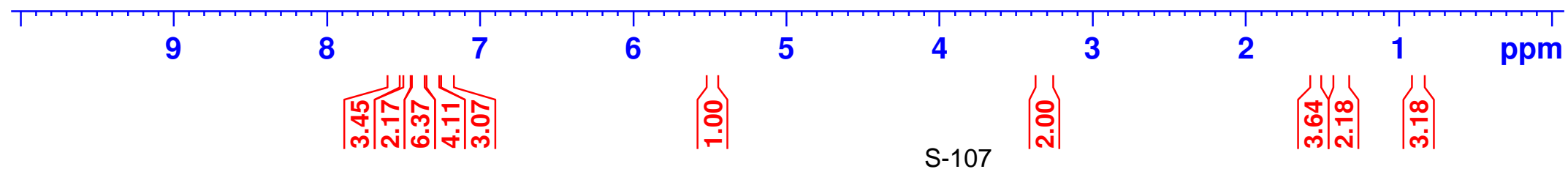




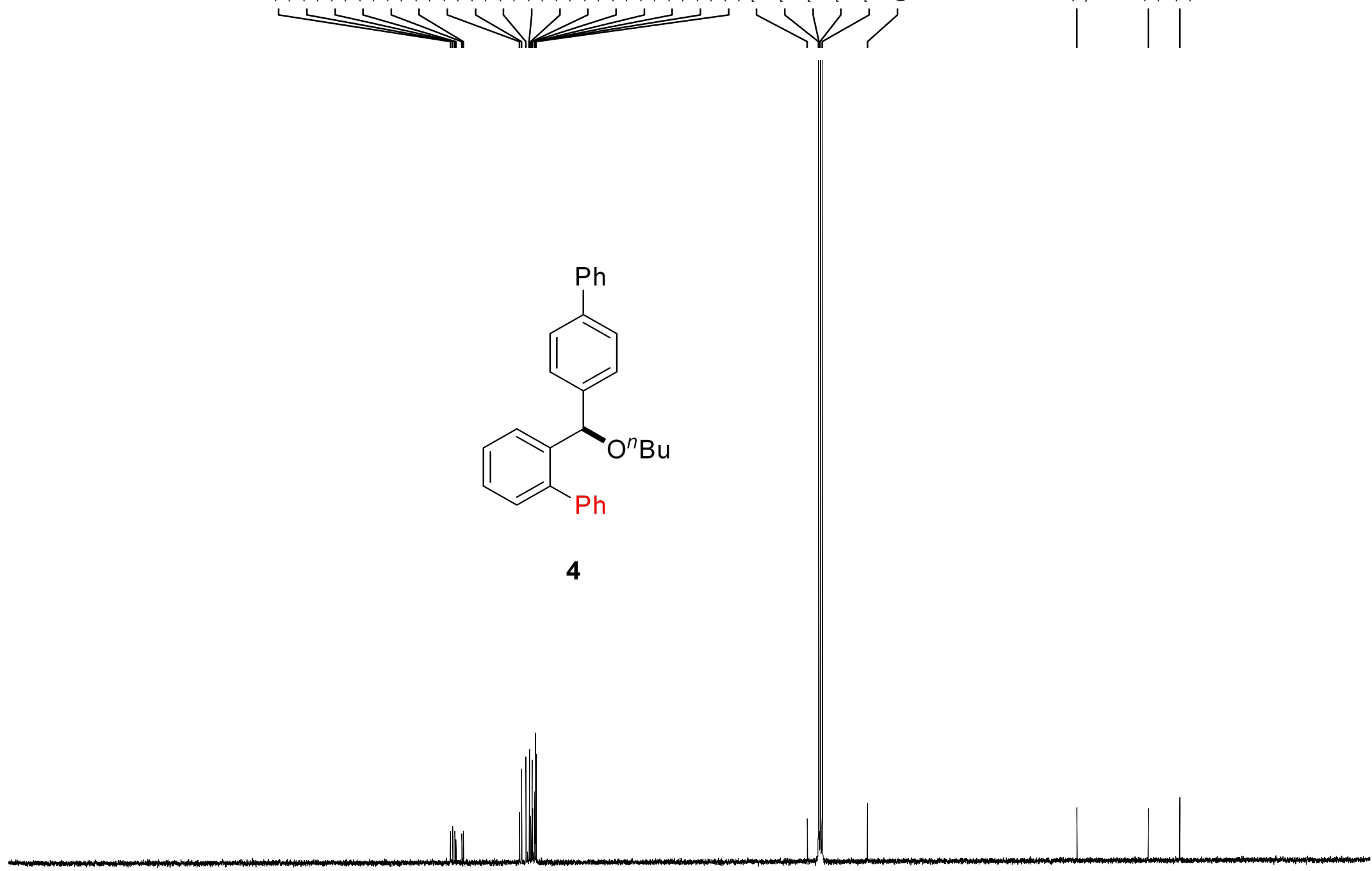

\section{BR $(1)$}

Current Data Parameters NAME lzw3124-C

EXPNO

PROCNO

3
1

F2 - Acquisition Parameters

Date 20150802

Time 14.07

INSTRUM spect

PROBHD $5 \mathrm{~mm}$ PABBO BB/

$\begin{array}{lr}\text { PULPROG } & \text { zgpg30 } \\ \text { TD } & 65536\end{array}$

$\begin{array}{lr}\text { SOLVENT } & 65536 \\ \text { CDCl3 } & \end{array}$

DS

SWH

$\begin{array}{lr}\text { SWH } & 24038.461 \mathrm{~Hz} \\ \text { FIDRES } & 0.366798 \mathrm{~Hz}\end{array}$ 1. $3631488 \mathrm{~Hz}$

CHANNEL $\mathrm{f} 1 \mathrm{l}========$ $100.6228298 \mathrm{MHz}$

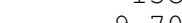
9.70 usec $\mathrm{SFO} 2$

NUC2

CPDPRG [2

PCPD2

PLW2

PLW12

$400.1316005 \mathrm{MHz}$

waltz 16 $11.99499989 \mathrm{~W}$ $0.34213999 \mathrm{~W}$ $0.27713001 \mathrm{~W}$

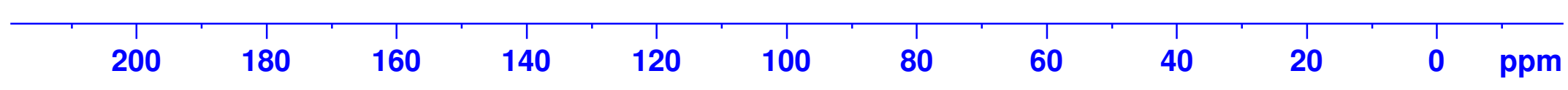

F2 - Processing parameters I 32768 SF $\quad 100.6127712 \mathrm{MHz}$

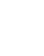




\section{sp $(->)$}

Current Data Parameters NAME

PROCNO

lzw3149-H

F2 - Acquisition Parameters

Date_

Time $\quad 19.26$

spect
INSTRUM

ROBHD

zgB 30
65536

$\begin{array}{ll}\text { TD } & 65536 \\ \text { SOLVENT } & \text { CDCl3 }\end{array}$

NS

SWH

FIDRES

AQ

$2 x^{\prime}$

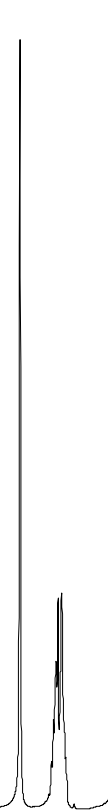

$8012.820 \mathrm{~Hz}$

$4.0894465 \mathrm{sec}$

34.77

62.400 usec 6.50 usec

DW
DE
TE

D 10

$$
1
$$

$==$

$\mathrm{SFO}$

NUC

P1
PLW1

CHANNEL $\mathrm{fl}========$

$400.1324710 \mathrm{MHz}$ $1 \mathrm{H}$

$11.99499989 \mathrm{~W}$

F2 - Processing parameters

SI 65536

$\begin{array}{ll}\text { SF } & 400.1300000 \mathrm{MHz}\end{array}$

WDW

SSB

EM

$0.30 \mathrm{~Hz}$

1.00

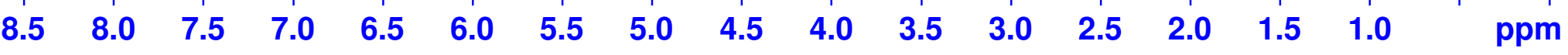

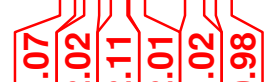

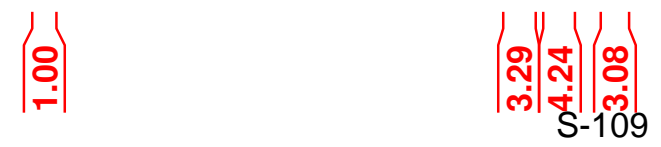



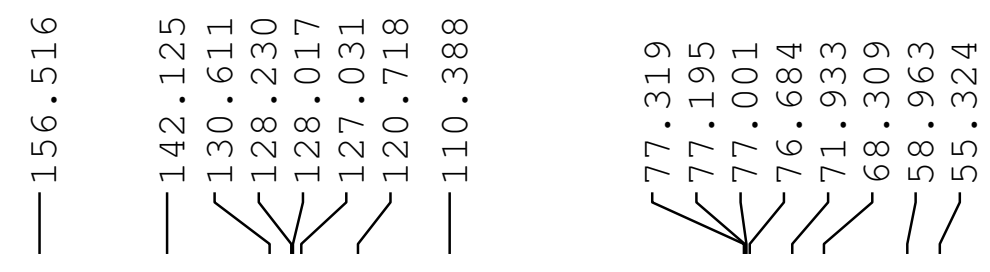

\section{BR XKRER $(1)$}

Current Data Parameters NAME

EXPNO

lzw3149-C<smiles>COCCOC(c1ccccc1)c1ccccc1OC</smiles>

$2 x^{\prime}$

F2 - Acquisition Parameters

Date_ 20150818

$\begin{array}{ll}\text { Time } & 19.29 \\ \text { INSTRUM } & \text { spect }\end{array}$

spect

PULPROG 5

zgpg30
65536

SOLVENT

NS

NS
DS
SWH

SWH

FIDRES

AQ

RG

DE

$\mathrm{DE}$

D1 1

D11

$======$

$\mathrm{SFO1}$
NUC1

P1
PLW1

CDC13

35
0

$24038.461 \mathrm{~Hz}$

$24038.461 \mathrm{~Hz}$
$0.366798 \mathrm{~Hz}$

$1.3631488 \mathrm{sec}$ 196.92

20.800 usec 6.50 usec

$2.00000 .8 \mathrm{~K}$

$0.03000000 \mathrm{sec}$

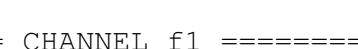
$100.6228298 \mathrm{MHz}$ $13 \mathrm{C}$
9.70 9.70 use

$46.98899841 \mathrm{~W}$

$$
\begin{aligned}
& === \\
& \mathrm{SFO} 2
\end{aligned}
$$

$\mathrm{SFO} 2$
$\mathrm{NUC2}$

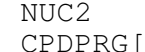

CPDPRG [

PCPD2
PLW2

PLW2

PLW13

NNEL f2 $========$ $400.1316005 \mathrm{MHz}$ $1 \mathrm{H}$

90.00 usec $11.99499989 \mathrm{~W}$ $0.34213999 \mathrm{~W}$ $0.27713001 \mathrm{~W}$

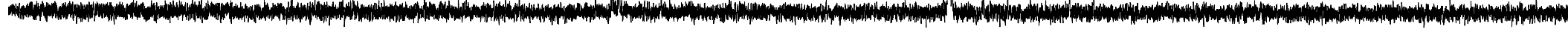

22 - Processing parameters

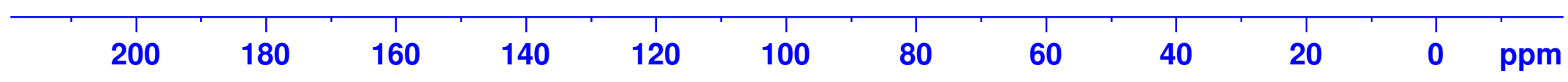

SI Processing parameters 32768

WDW

$100.6127795 \mathrm{MHz}$ 
ㄱํ

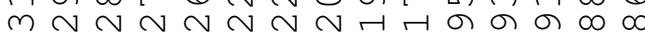

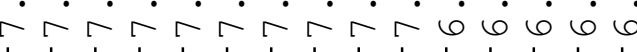

$\longrightarrow$

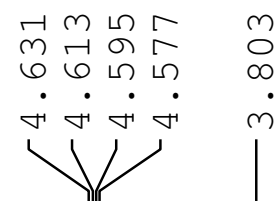

$\begin{array}{ll}\text { न } & \text { の } \\ 6 & \curvearrowleft\end{array}$

-

V

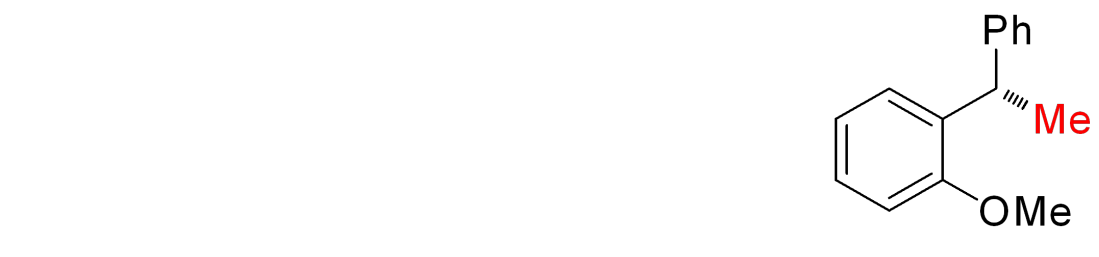

5

\section{en $(1)$}

Current Data Parameters NAME

EROCNO

lzw3147-H

F2 - Acquisition Parameters

Date__ 20150818

Time $\quad 13.21$

$5 \mathrm{~mm}$ spect

PULPROG

$\mathrm{Zg} 30$
65536

$\begin{array}{ll}\text { TD } & 65536 \\ \text { SOLVENT } & \text { CDCl3 }\end{array}$

NS

SWH

FIDRES

$\mathrm{AQ}$

$\mathrm{AQ}$
$\mathrm{RG}$

DW

$\mathrm{DE}$

TE

TD 0

$===$

$\mathrm{SFO}$

$\mathrm{NUC}$

$\mathrm{CDC} 13$
8

$8012.820 \mathrm{~Hz}$

$0.122266 \mathrm{~Hz}$

$4.0894465 \mathrm{sec}$ 103.52

62.400 usec 6.50 use
$295.9 \mathrm{~K}$

$1.00000000 \mathrm{sec}$

$$
1
$$

CHANNEL $\mathrm{f} 1 \mathrm{l}========$ $400.1324710 \mathrm{MHz}$

- Processing parameter

SI $\quad 65536$

SF $\quad 400.1300000 \mathrm{MHz}$

WDW

$S S B$

$\mathrm{EM}$

$0.30 \mathrm{~Hz}$

1.00

7

|

5

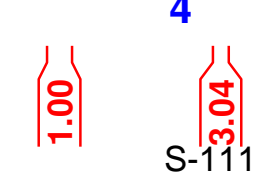

3

2

1

ppm

$\left|\frac{m}{m}\right|$ 


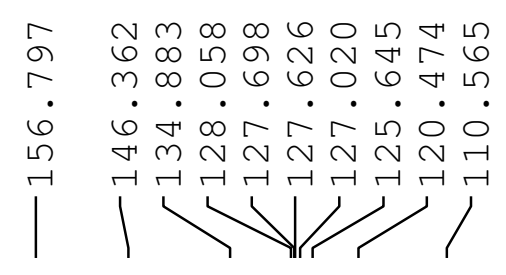

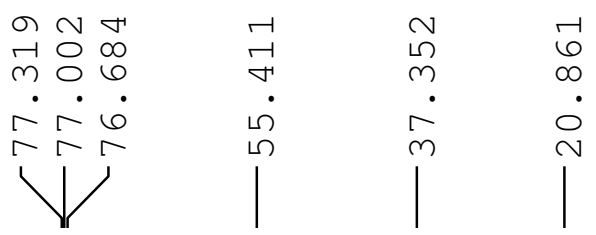
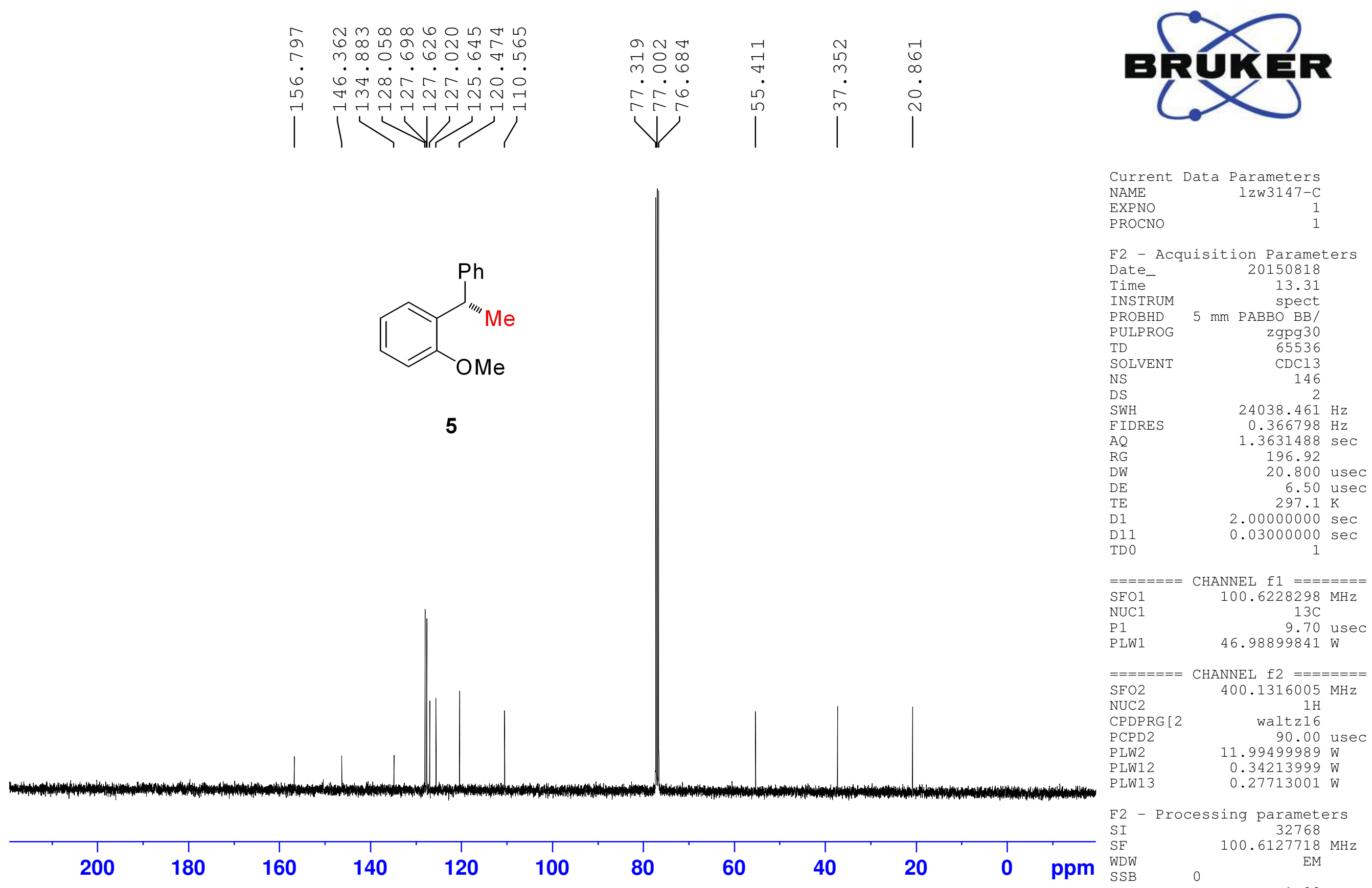

F2 - Processing parameters SI 32768

180

